\title{
Ultra-light dark matter
}

\author{
Elisa G. M. Ferreira ${ }^{1,2}$ (D)
}

Received: 17 May 2020 / Accepted: 4 June 2021 / Published online: 9 September 2021

(C) The Author(s) 2021

\section{Abstract}

Ultra-light dark matter is a class of dark matter models (DM), where DM is composed by bosons with masses ranging from $10^{-24} \mathrm{eV}<m<\mathrm{eV}$. These models have been receiving a lot of attention in the past few years given their interesting property of forming a Bose-Einstein condensate (BEC) or a superfluid on galactic scales. BEC and superfluidity are some of the most striking quantum mechanical phenomena that manifest on macroscopic scales, and upon condensation, the particles behave as a single coherent state, described by the wavefunction of the condensate. The idea is that condensation takes place inside galaxies while outside, on large scales, it recovers the successes of $\Lambda \mathrm{CDM}$. This wave nature of DM on galactic scales that arise upon condensation can address some of the curiosities of the behaviour of DM on small-scales. There are many models in the literature that describe a DM component that condenses in galaxies. In this review, we are going to describe those models, and classify them into three classes, according to the different non-linear evolution and structures they form in galaxies: the fuzzy dark matter (FDM), the self-interacting fuzzy dark matter (SIFDM), and the DM superfluid. Each of these classes comprises many models, each presenting a similar phenomenology in galaxies. They also include some microscopic models like the axions and axion-like particles. To understand and describe this phenomenology in galaxies, we are going to review the phenomena of BEC and superfluidity that arise in condensed matter physics, and apply this knowledge to DM. We describe how ULDM can potentially reconcile the cold DM picture with the small-scale behaviour. These models present a rich phenomenology that is manifest in different astrophysical consequences. We review here the astrophysical and cosmological tests used to constrain those models, together with new and future observations that promise to test these models in different regimes. For the case of the FDM class, the mass where this model has an interesting phenomenology on smallscales $\sim 10^{-22} \mathrm{eV}$, is strongly challenged by current observations. The parameter space for the other two classes remains weakly constrained. We finalize by showing

\footnotetext{
$\bowtie \quad$ Elisa G. M. Ferreira elisagmf@mpa-garching.mpg.de

1 Max-Planck-Institut für Astrophysik, Karl-Schwarzschild-Str. 1, 85748 Garching, Germany

2 Instituto de Física, Universidade de São Paulo, C.P. 66318, São Paulo, SP 05315-970, Brazil
} 
some predictions that are a consequence of the wave nature of this component, like the creation of vortices and interference patterns, that could represent a smoking gun in the search of these rich and interesting alternative class of DM models.

\section{Keywords Ultra-light dark matter · Fuzzy dark matter · Superfluid dark matter · Bose-Einstein condensate $\cdot$ Superfluid}

\section{Contents}

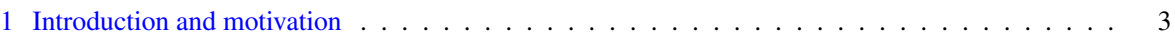

2 Small-scale challenges of cold dark matter . . . . . . . . . . . . . . . . . . . . . . . . . 9

2.1 Dark matter halos and substructures . . . . . . . . . . . . . . . . . . . 11

2.2 Discrepancies in comparison with observations . . . . . . . . . . . . . . . 13

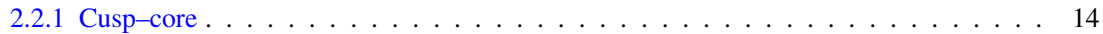

2.2 .2 Missing satellites . . . . . . . . . . . . . . . . . . . . . . . . . . . . . . . . . .

2.2 .3 Diversity vs. regularity: scaling relations . . . . . . . . . . . . . . . . . . . . . . . . . . . . . . .

2.2 .4 What the small-scales tell us . . . . . . . . . . . . . . . . . . . . . 20

3 Bose-Einstein Condensation and Superfluidity . . . . . . . . . . . . . . . . . . . . 23

3.1 Non-interacting ideal gas . . . . . . . . . . . . . . . . . . . . . . . . . 25

3.2 Landau's superfluid model and criteria for superfluidity . . . . . . . . . . . . . . . . . . . . . . . . . . . 28

3.3 Weakly interacting Bose gas superfluid . . . . . . . . . . . . . . . . . . . . . . . . . . . . . . . . . .

3.3 .1 Field theory description . . . . . . . . . . . . . . . . . . . . . 39

3.4 Effective field theory of a superfluid . . . . . . . . . . . . . . . . . . . . . . . . . . . . . . . . . . .

3.5 Rotating superfluid - quantum vortices . . . . . . . . . . . . . . . . . . . 52

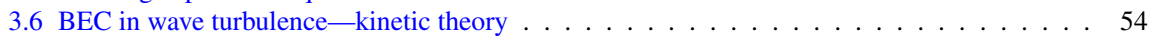

3.7 Summary and discussion: what is a condensate? . . . . . . . . . . . . . . . . . . 59

4 Ultra-light dark matter . . . . . . . . . . . . . . . . . . . . . . . . . . 60

4.1 FDM and SIFDM . . . . . . . . . . . . . . . . . . . . . . . . . . 68

4.1 .1 Formation: ALPs . . . . . . . . . . . . . . . . . . . . . . . 69

4.1 .2 Cosmological evolution . . . . . . . . . . . . . . . . . . . . . . . . . . 70

4.1 .3 Evolution on small-scales . . . . . . . . . . . . . . . . . . . . 74

4.1 .4 Description of the condensate . . . . . . . . . . . . . . . . 76

4.1 .5 Discussion . . . . . . . . . . . . . . . . . . . . . 91

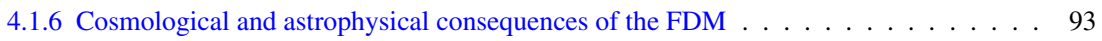

4.1 .7 Addressing the small-scale challenges . . . . . . . . . . . . . . . . . . . . 108

4.2 DM superfluid . . . . . . . . . . . . . . . . . . . . . . . . 111

4.2.1 Conditions for DM condensation . . . . . . . . . . . . . . . . . . 112

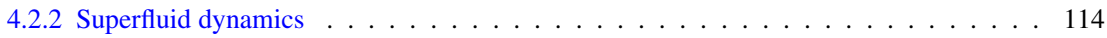

4.2 .3 Halo profile . . . . . . . . . . . . . . . . . . . . . . . . 117

4.2 .4 Observational consequences . . . . . . . . . . . . . . . . . . . . . . . . . . . . . . . . . . . . . . . . 120

4.2 .5 Validity of the EFT . . . . . . . . . . . . . . . . . . . . . . . . . . . . . . . . . 127

4.2 .6 Relativistic completion . . . . . . . . . . . . . . . . . . . . . . . . 130

4.2 .7 Cosmology . . . . . . . . . . . . . . . . . . . . . . . . 131

4.3 Simulating ULDM models . . . . . . . . . . . . . . . . . . . . . . . . . . . . . . . . . . . . . . . . . . . . . . . . . . . .

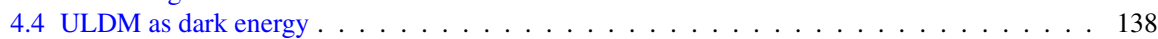

4.4 .1 Fuzzy DM . . . . . . . . . . . . . . . . . . . . . . 138

4.4.2 Superfluid DM-unified superfluid dark sector . . . . . . . . . . . . . . . . . . 139

5 Cosmological and astrophysical constraints, and new windows of observation . . . . . . . . . . . 141

5.1 Cosmological constraints: CMB and LSS . . . . . . . . . . . . . . . . . . . . . . . . . . . . . . . . . . . . . . . . . .

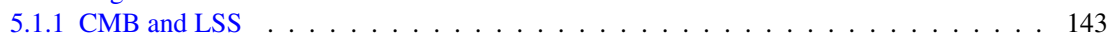

5.1 .2 Lyman $\alpha \ldots \ldots \ldots \ldots \ldots \ldots \ldots$

$5.1 .321 \mathrm{~cm}$ cosmology . . . . . . . . . . . . . . . . . . . . 146

5.2 Astrophysical constraints and new windows of observation . . . . . . . . . . . . . . 149

5.2.1 Local Milky Way observables and stellar streams . . . . . . . . . . . . . . . . . . . 149 
5.2 .2 Substructure-strong lensing . . . . . . . . . . . . . . . . . . . . . . . 157

5.3 UV luminosity function . . . . . . . . . . . . . . . . . . . . . . 158

5.4 Black hole superradiance . . . . . . . . . . . . . . . . . . . . . . . . . . . . . . . . . . . . 159

5.5 Probing the wave nature of ULDM $\ldots \ldots \ldots \ldots \ldots$

5.5 .1 Vortices . . . . . . . . . . . . . . . . . . . . . . . . . 161

5.5 .2 Interference fringes . . . . . . . . . . . . . . . . . . . . . . . . . 164

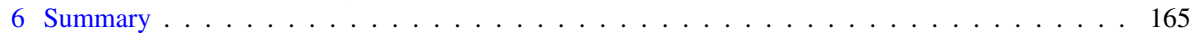

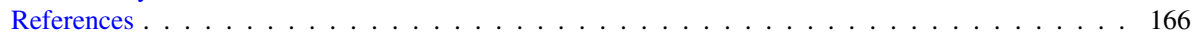

\section{Introduction and motivation}

An overwhelming amount of observational data provides clear and compelling evidence for the presence of dark matter (DM) on a wide range of scales. This component, which is believed to be responsible for the "missing" mass in our universe, is the main ingredient for all the structures we have in our universe. This is one of the oldest unsolved problems in cosmology, being traced back to the 1930s (Zwicky 1933; Bertone and Hooper 2018), and also one of the best measured ones. The evidence for dark matter first emerged from the study of the rotation curves of galaxies. From pioneering studies (Rubin and Ford 1970), it was already evident that the amount of matter necessary to fit the flat observed rotation curves did not match the theoretical curves predicted, assuming Newtonian mechanics and taking into account only the visible matter present in those galaxies. Dark matter was proposed as an additional (non-luminous) component to explain this discrepancy.

Nowadays, the evidence for dark matter comes from precise measurements on a wide range of scales. From sub-galactic and galactic scales, to clusters, going up to the large-scale structure (LSS). On cosmological scales, the observed anisotropies of the cosmic microwave background (CMB) (Ade et al. 2016), together with data from Type Ia Supernovae, determine the total energy density of matter with high precision. This together with the bounds on the abundance of the light chemical elements from Big Bang Nucleosynthesis, which constrains the amount of baryonic matter in the universe, strongly shows the need for a clustering component of non-baryonic ${ }^{1}$ origin, that does not interact (strongly) with photons, and that dominates the matter content of the universe, accounting for approximately $85 \%$ of all matter. The same non-luminous and clustering component is necessary to explain the structures we see in our universe today, as is evident in observations of the large-scale structure of our universe (Anderson et al. 2014; Tegmark et al. 2004).

With all this evidence coming from precise astrophysical and cosmological observations, cosmologists have converged to a phenomenological model to describe our universe, the $\Lambda \mathrm{CDM}$ model. This model is currently the concordance model of cosmology and it accumulates a number of observational successes. It exhibits outstanding agreement with current cosmological observations (Anderson et al. 2014), which is manifested in the parameters of this model being constrained at the percent and subpercent level. This incredibly simple model is described by only six parameters and parametrizes a large amount of the universe's history. It describes a universe that is flat and seeded by nearly scale invariant perturbations, composed of baryons, which

\footnotetext{
${ }^{1}$ We are going to see later that there are some "baryonic" candidates for DM.
} 
80 orders of magnitude

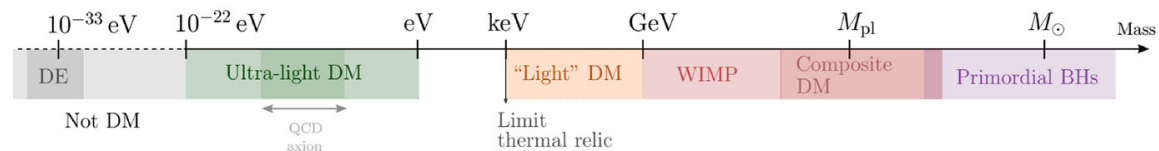

Fig. 1 Sketch (not to scale) of the huge range of possible DM models that have been conceived. They span many orders of magnitude in mass, with DM represented by very distinct phenomena, ranging from new elementary particles to black holes

amount to approximately $5 \%$ of the energy density of the universe, a small radiation component, but in its majority is composed of two unknown ingredients. The energy budget of the universe is dominated $(\sim 70 \%)$ by a component responsible for the current accelerated expansion of the universe called dark energy, and a clustering component, the dark matter, making up to $\sim 25 \%$ of our universe. These large-scale observations give a coarse-grained description of these non-baryonic components in the hydrodynamical limit where dark matter is described as a perfect fluid with very small pressure $(w \approx 0)$ and sound speed, $c_{\mathrm{s}} \approx 0$, that does not interact, at least strongly, with baryonic matter. Dark energy is parametrized by a cosmological constant, the simplest model for the present accelerated expansion of our universe.

Therefore, within $\Lambda \mathrm{CDM}$, the Cold Dark Matter (CDM) paradigm emerged from the large-scale observations and describes the component responsible for the formation of the structures of our universe through gravitational clustering. In the CDM model, DM is described by a perfect fluid that must be massive, sufficiently cold, which means non-relativistic at the time of structure formation, and collisionless to explain the observational data on large linear scales. This coarse-grained description of a CDM is very successful in fitting the linear, large-scales observations from the CMB, LSS, to clusters, and general properties of galaxies.

However, even though we know the hydrodynamical properties of DM on largescales to a very high precision, the microphysics of the DM component remains unknown. This allows for the creation of a plethora of possible models of DM. Those models recover the large scale properties of CDM, but invoke very different objects and phenomena to play the role of DM.

This incredible variety of viable models of DM can be seen in the huge range of masses those models cover, as shown in Fig. 1. This figure shows many different broad classes of DM models, and each of which might contain many different specific models. It spans more than 80 orders of magnitude and shows very different hypothesis for DM, from new elementary particles, to composite objects (Jacobs et al. 2015; Khlopov 2019), up to astrophysical size primordial black holes (for a review on recent bounds, see (Carr et al. 2020; Carr and Kuhnel 2020)). This shows us that although we have gathered a lot of knowledge about the gravitational properties of DM, the nature of DM is still elusive, with the current data still allowing a huge amount of highly different models.

The possibility that dark matter could be a long lived particle is very appealing, especially, if these candidates are expected candidates from extensions of the standard model of particle physics. One class of models that became the preferred candidates for 
the DM particles are WIMP, weakly interacting massive particles, which represent new elementary particles that interact with baryons not only gravitationally but also through the weak force or a new force of comparable strength (Roszkowski et al. 2018; Lin 2019). The strong motivation for this candidate is because if it is thermally produced in the early universe, the relic abundance of particles that have mass of the order of the electroweak scale, and a coupling of order one, corresponds precisely to the abundance of DM in our universe. The possibility that WIMP could also be discovered by direct detection experiments is also an important motivation to search for this candidate. There is a great experimental effort to constrain the properties of WIMP DM with the parameter space being very restricted over the past few years. Given the complex phenomenology from the possible models of WIMP DM and their interaction with the standard model particles, the translation of those bounds to the exclusion of WIMP models is not straightforward. The cosmological and astrophysical behaviour of all the classes of WIMP models is similar to CDM, so the avenue to probe this scenario is through direct, indirect and collider experiments (for a complete review of all the searches, current and projected limits on WIMP detection for both spin-dependent and spin-independent models, together with indirect detection and collider searches, see (Arcadi et al. 2018).)

Another candidate that comes from extensions of the standard model of particle physics is the QCD axion. The axion was introduced to address the strong CP problem of quantum chromodynamics (QCD) (Peccei and Quinn 1977; Weinberg 1978; Wilczek 1978). The axion can be used in many different contexts in cosmology, including as a candidate for DM. The QCD non-perturbative effects induce a potential for the axion. During the radiation dominated period, the QCD axion starts to oscillate at the bottom of its potential and the axions behaves like dust, contributing to the energy density of the universe as non-relativistic matter. The QCD axion couples weakly to the standard model, which motivated an experimental effort for its direct detection (see these references for a review of axion direct detection searches (Di Luzio et al. 2020; Sikivie 2020; Graham et al. 2015a; Battesti et al. 2008)).

Although we have these very well motivated candidates from particle physics, we still have no conclusive evidence for electroweak or other non-gravitational interactions for dark matter. All the knowledge we have about dark matter is gravitational. We know that CDM describes the behaviour of DM very well on large-scales. However, this beautiful and simple coarse grained description of DM as the CDM is challenged by some curiosities that appear on small scales.

As the observations and simulations of the small non-linear scales and galactic scales improve, a number of challenges have emerged for this coarse grained description from $\Lambda \mathrm{CDM}$. These discrepancies have been around for decades, such as the cusp-core problem, the missing satellite problem and the too big to fail problem. A particularly curious challenge is the regularity/diversity of rotation curves. One thing that is surprising about galaxies is that they are extremely diverse, but at the same time they are incredibly regular. This fact is manifest in several empirical scaling relations, such as the well-known Baryonic Tully-Fisher relation (BTFR; McGaugh 2005, 2008). The BTFR shows the correlation between the total baryon mass (including stars and gas) of the galaxy with the asymptotic rotation velocity in galaxies. The measured BTFR follows a scaling relation different from the one predicted by $\Lambda \mathrm{CDM}$, 
and it holds for a range of 6 orders of magnitude in mass, with very small scatter. The significance of these discrepancies is disputed and addressing these challenges is an active field of research. Those challenges emerge on scales where baryonic physics is relevant and simulations including several baryonic effects have been perfected pointing in the direction that baryons could possibly explain some of these observations within $\Lambda \mathrm{CDM}$.

As the physics of these baryonic processes is complex and as there is no final consensus about the status of theses discrepancies, an alternative explanation for these discrepancies on small-scales could be that DM is not the usual CDM, but a component that has different phenomenology on small-scales. Even setting aside the small-scale problems, given that the observational constraints on these scales are less strong than on cosmological scales, the dynamics on small-scales can offer a chance to probe the properties of DM in the hope to help find the microphysics of this component. Therefore, the small-scales are a good laboratory to probe the nature of DM models that have impacts on astrophysical scales.

The simplest modifications of the DM paradigm that have a different phenomenology on small-scales, and can potentially address some of the small-scales discrepancies is the warm dark matter (WDM) model (Colin et al. 2000). In this model, DM has a small mass leading to a thermal velocity dispersion, modifying its behaviour on astrophysical scales while maintaining the large-scale predictions of CDM. Even with a small velocity, DM free streams out of potential wells and is enough to suppress the formation of small scale structures addressing some of the small-scale problems. Another popular model inspired by those discrepancies is the self interacting DM (SIDM) (Spergel and Steinhardt 2000), where the DM particles have a self-interaction in a way to also suppress the formation of structures on small-scales.

In the past few years, another class of alternative models has (re)emerged as an appealing class of DM models given their rich phenomenology on small-scales. These are the ultra-light dark matter models (ULDM), where DM is composed by ultra-light bosons with masses in the range $10^{-24} \mathrm{eV}<m<\mathrm{eV}$. Given the small masses of these bosons, DM forms a condensate or a superfluid on galactic scales. The idea is that the wave nature of DM on galactic scales provides a non-CDM behavior which leads to a different and rich phenomenology for DM on those scales. On large scales, DM behaves as CDM, although with different initial conditions for the ULDM in comparison to CDM, maintaining the observable successes of CDM on those scales.

There are many different realizations of this interesting non-CDM phenomenology on small-scales. Depending on the modelling of the ULDM, these produce distinct condensate structures and lead to a different phenomenology. There are many different models in the literature describing these possibilities. We classify them in this review into three categories, according to the different condensate structure they form. These three classes are the fuzzy dark matter, when the ultra-light scalar field system is only subjected to gravity; the self-interacting Fuzzy DM when the system also presents (weakly) self-interaction, and superfluid DM, where DM forms a superfluid on galactic scales.

This classification is general and based only on the non-linear structure it forms in the halo of galaxies, which is a consequence of the non-relativistic theory they describe. These can be purely phenomenological models of ULDM on small-scales, 
in the absence of a microscopic description. These classes also contain microscopic scalar field theories like QCD axion, axions (coming from other origins) or axion like particles (ALP), which are part of the FDM class. Each of these categories have different properties which lead to different astrophysical consequences, that can be probed by current and future astrophysical observations. For this reason, ULDM models have regained interest in the community in the past few years, with new and exciting experimental effort to probe many aspects of the small astrophysical scales, opening the avenue to test these models and answer some questions about the nature of DM.

\section{Motivation for this review and detailed plan}

This review has the goal of giving an overview of the ultra-light dark matter (ULDM) candidates, focusing in their gravitational effects and mostly in their different phenomenology on small-scales.

There are many very good and complete reviews in the literature describing specific models of ULDM or the microscopic models that can be part of the ULDM class like. There are many reviews of axions in cosmology (Sikivie 2008; Arvanitaki et al. 2010; Wantz and Shellard 2010; Kim and Carosi 2010; Kawasaki and Nakayama 2013; Marsh 2016a) and ALPs (Ringwald 2014; Arias et al. 2012; Graham et al. 2015a; Marsh 2018; Niemeyer 2019; Powell 2016). Axions and ALPs have a whole rich phenomenology of its interactions to the standard model that will not be explored here, but that can be seen in the following reviews (Marsh 2016a; Graham et al. 2015a). The FDM model also has a huge body of literature with many excellent reviews like (Hui et al. 2017; Suárez et al. 2014; Ureña-López 2019).

We propose to do something different in this review. Instead of studying one single model or a specific microscopic theory, we study many ULDM models interested in the gravitational phenomenology that these models present. We study the ULDM models by dividing them into classes according to their dynamics on small-scales. The three classes proposed in this review encompass many of the models cited above, with the inclusion of (weakly) self-interacting models and the DM superfluid model. We believe this classification is instrumental and shows the general behaviour and phenomenology that each of these model have inside each class. Therefore, we hope to bring not only a new classification that encompasses many of the models present in the literature, but also to include new models, trying to make a big overview of the entire class of ULDM models.

Another new feature this review brings is a brief review of BEC and superfluidity, and the different descriptions of these phenomena. Condensation in each of these classes might arise in a different way, given their different descriptions. Bose-Einstein condensation and superfluidity are very well understood and well studied macroscopic quantum phenomena in condensed matter physics, being largely studied theoretically and experimentally. However, these phenomena are not so well understood in gravity. Therefore, understanding their definition, description and differences is particularly important to understand if condensation arises in theses models, and the difference in the condensate structure that is expected to form in each of the classes of ULDM models.

I take this opportunity to also discuss briefly the different views in the literature about the formation of a condensate and the scales where this effect takes place. 
With that, we aim to give a general picture of the state of the field to date, trying to describe all the classes of ULDM present in the literature. We hope this review can be a resource to researcher entering this exciting field.

The review is organized as follows. First, in Sect. 2, we start by describing the small scale challenges of $\Lambda \mathrm{CDM}$, as a motivation to show the discrepancies these alternative models of DM aim to address. The goal of this section is not only to show the problems that some of the ULDM models might solve, but also to introduce the reader into some of the concepts of galactic astrophysics. In this way, the reader can understand some of the interesting phenomenology that the ULDM models have on small-scales that differ from the ones predicted by the CDM paradigm. Next, in Sect. 3, we introduce the basic concepts of the quantum phenomena of BEC and superfluids. In this section, we describe these phenomena, describe approximations and and the structures formed in those system with and without rotation, all in the context of condensed matter physics where they are well defined, understood and tested. The goal is to give a sound basis to the reader so they can understand with a critical view how these concepts can be applied to the case of DM in the next section, given the analogies, approximations and generalizations done in the literature of ULDM. Following this we are ready to describe the main topic of the review, the ULDM models in Sect. 4. We start by describing the three classes that we propose to classify the models of ULDM based on the type of non-linear they describe. We then talk about the fuzzy DM and the self-interacting BEC DM models, showing the conditions for them to condense on galactic scales. We then focus on the fuzzy $D M$ model, showing how and in which conditions the model attempts to solve the small-scale challenges, and the interesting astrophysical consequences this class presents. We then talk about the superfluid DM model describing its condensation on galactic scales, the formation of the superfluid core and its observational consequences. We also discuss the stability of this construction, and its possible extension to cosmology. The constraints in these models and new windows of observations of the effects of these models are discussed in Sect. 5. We will constraints from different observations. In the case of the FDM, the current bounds show that the mass range where an interesting phenomenology is expected on small-scales is strongly constrained. We conclude the review summarizing our discussion.

Since there is no unique literature this review is based on, but a series of reviews and articles referring to specific topics, the main references used are cited in the corresponding sections. The only exception is Sect. 2 that is based mainly in the following reviews (Bullock and Boylan-Kolchin 2017; Del Popolo and Le Delliou 2017; Famaey and McGaugh 2012).

Conventions In the entire review, natural units are used, where $c=\hbar=1$, unless stated otherwise. The exception is Sect. 3 where all the $\hbar$ factors are present. With that, the reduced Planck mass is given by $M_{\mathrm{pl}}^{2}=1 / 8 \pi G$, where $G$ is the Newtonian gravitational constant. Unless stated otherwise, the metric signature used is $(+,-,-,-)$, and Greek letters are indices going from $\mu, v=0, \ldots, 3$. Sometimes for simplicity we describe partial derivatives as $\partial_{\mu}=\partial / \partial x^{\mu}$. In the text gray boxes bring definitions necessary for the understanding of the topics in the section or following sections. 
Frames text and equations refer to important results or discussions that we would like to highlight.

\section{Small-scale challenges of cold dark matter}

In the concordance model of cosmology, DM is described by the CDM paradigm. This hydrodynamical description for DM is in very good agreement with observations from large-scales. This can be seen in the power spectrum $(P(k))$, which is the Fourier transform of the two-point correlation function of the matter density perturbations,

$$
\Delta^{2}(k)=\frac{1}{2 \pi^{2}} k^{3} P(k)
$$

represented here by the dimensionless power spectrum where $k$ is the wavenumber of the fluctuation, shown in Fig. 2. The large-scales (around $k \lesssim 0.1 \mathrm{Mpc}^{-1}$ ), measured by the CMB and LSS galaxy surveys, show a good compatibility with the CDM model. This agreement is also robust for the non-linear intermediary scales $\left(k \sim\left[10^{-1}-\right.\right.$ $10] \mathrm{Mpc}^{-1}$ ) with constrains from clusters, weak lensing and Ly- $\alpha$ forest. As we go to smaller and highly non-linear scales $\left(k \gtrsim 10 \mathrm{Mpc}^{-1}\right.$ equivalent to $\left.M \lesssim 10^{10} M_{\odot}\right)$, these constraints are less strong, and might retain important information about possible deviations from the CDM paradigm. We can see on the right side of Fig. 2 on galactic and subgalactic scales, different models of DM would behave very differently from the expected linear behaviour of CDM and this could be probed by the observations on those scales (Zavala and Frenk 2019; Kuhlen et al. 2012).

On small-scales, the formation of structures is highly non-linear and the evolution of structures is studied using large numerical simulations. In the past few years, those simulations improved in size and precision, simulating the cosmological and smallscales. But when compared to the observations of galaxies, a number of discrepancies emerged, revealing some curious behaviour on small scales. Given the enormous success of the concordance model, these discrepancies attract a lot of interest of the community. They might represent that we need to better take into account the astrophysical processes that happen inside those regions, which indeed have a complex dynamics. Or this might indicate that the CDM model is not good to describe the physics on small-scales and the coarse grained CDM paradigm needs to be revised. An even a more radical approach would be to modify gravity on smaller scales.

In this section, we present very concisely the theory of non-linear structure evolution. We show how the numerical predictions assuming the concordance model might be in tension with the current observations of galaxies. These tensions are seen in the counts and density of low-mass objects, and in the scaling relations that show the tight regularity that galaxies present. We highlight in this section some of the concepts that are going to be used in the ULDM section and that might not be too familiar for researchers from fields of dark matter phenomenology and cosmology. 


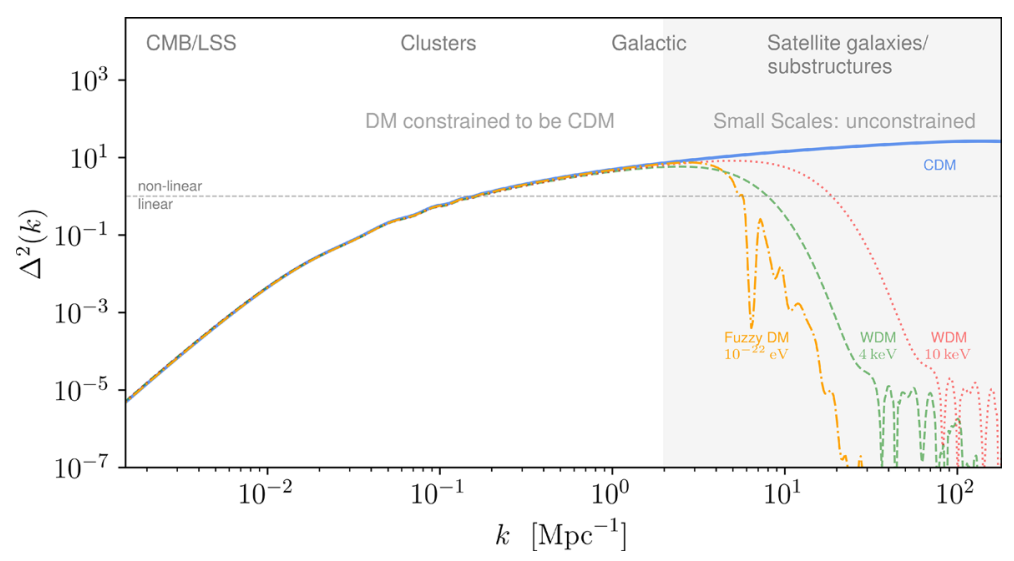

Fig. 2 In this figure, inspired by Kuhlen et al. (2012), we show how the dimensionless power spectrum can be probed by many large-scale and small-scale observables, which can be seen as a function of the wavenumber $k$. The solid line shows the linear dimensionless power spectrum coming from a $\Lambda \mathrm{CDM}$ universe. To show how the small-scales might reveal different behaviour for different DM components, we show the linear power spectrum of warm DM (WDM) with mass of $10 \mathrm{keV}$ (red dotted line), WDM with mass of $4 \mathrm{keV}$ (green dashed line), and for fuzzy DM with mass $10^{-22} \mathrm{eV}$ (orange dash-dotted line). The gray dotted horizontal line represents the limit from linear to non-linear regime, where $\Delta \sim 1$. The power spectra for $\Lambda \mathrm{CDM}$ and for WDM were generated using the Boltzmann code CLASS (Lesgourgues 2011; Lesgourgues and Tram 2011), and for the fuzzy DM using AxionCAMB (Lewis et al. 2000; Hložek et al. 2015)(The parameters used to generate these power spectra were: $\Omega_{b} h^{2}=0.022, \Omega_{c} h^{2}=0.12, h=0.67$, $n_{s}=0.96, A_{s}=2.2 \times 10^{-9}$, and $\tau=0.09$.)

Summary of scales and galaxies ${ }^{2}$

Galaxy clusters: Largest gravitationally bound systems in the universe, with masses $\sim 10^{14}-10^{15} M_{\odot}$ (equivalent to $k \sim[1.5-6] \times 10^{-1} \mathrm{Mpc}^{-1}$ ), containing hundreds of galaxies, hot gas and mostly DM.

Milky-Way $(M W)$ galaxy: MW is a barred spiral galaxy and part of the Local Group of galaxies with virial mass $\sim 10^{12} M_{\odot}$. It has a stellar disk of approximately $30 \mathrm{kpc}$ in diameter and $0.3 \mathrm{kpc}$ thick, and $v_{\text {vir }} \sim 100 \mathrm{~km} / \mathrm{s}$ (virial velocity, defined below), with the halo of the MW being hundreds of $\mathrm{kpc}$ in size.

\section{Dwarf galaxies:}

Dwarf galaxies are low luminosity, small size galaxies, with stellar masses $\left(M_{\star}\right)$ smaller than $10^{9} M_{\odot}$ and virial mass for the halo smaller than $10^{11} M_{\odot}$. Regarding their mass, they can be further divided into: Bright dwarfs $\left(M_{\star} \approx\right.$ $\left.M \sim 10^{7-9} M_{\odot}, M_{\text {vir }} \approx M \sim 10^{11} M_{\odot}\right)$, classical dwarfs $\left(M_{\star} \approx M \sim\right.$ $\left.10^{5-7} M_{\odot}, M_{\text {vir }} \approx M \sim 10^{10} M_{\odot}\right)$, and ultra-faint dwarfs $\left(M_{\star} \approx M \sim\right.$ $\left.10^{2-5} M_{\odot}, M_{\mathrm{vir}} \approx M \sim 10^{9} M_{\odot}\right)$. Regarding their characteristics, they can be divided into ellipticals, spheroidal and irregulars, that contain gas and star formation. 
Dwarf Spheroidals (dSphs): Type of dwarf galaxy with a close to spheroidal shape, they have low-luminosity with a very small quantity of gas and dust, and no recent star formation. They present a large amount of DM and are usually the satellites.

\subsection{Dark matter halos and substructures}

A halo can be described as a virialized spherical mass concentration of dark matter. Halos are formed by gravitational collapse of a non-linear overdense regions that stopped expanding to collapse into a sphere in virial equilibrium. ${ }^{3}$ The virialization of the halo happens through violent relaxation, where the DM particles scatter on small fluctuations of the gravitational field present in this distribution, taking a time $t_{\text {dyn }}$, the dynamical time, to fully cross the sphere. Once this process is completed, at $t_{\text {coll }}$, the dark matter halo has a radius approximately $1 / 6$ of the radius of the region it collapsed from, and average density (Schneider 2015)

$$
\langle\rho\rangle=\left(1+\delta_{\text {vir }}\right) \bar{\rho}\left(t_{\text {coll }}\right),
$$

where $\bar{\rho}$ is the mean density, and $\left(1+\delta_{\text {vir }}\right) \approx 178 \Omega_{\mathrm{m}}^{-0.6}$. Given this, the dark matter halo is defined as the spherical region where the density is approximately 200 times the critical density of the universe at a given redshift, with mass given by

$$
M_{200}=\frac{4 \pi}{3} R_{200}^{3} 200 \rho_{\mathrm{cr}},
$$

where $\rho_{\text {cr }}=3 H^{2}(z) /(8 \pi G)$. The virial velocity is given by the mean circular velocity at the virial radius, $V_{200}^{2} \equiv G M_{200} / R_{200}$. With that, one can express the evolution of the mass and virial radius with respect to $V_{200}: M_{200}=V_{200}^{3} / 10 G H(z)$ and $R_{200}=$ $V_{200} / 10 H(z)$. We can see from these expressions that halos that form early in the evolution of the universe are less massive, while late-forming halos are more massive and larger.

This definition is not unique and depends on the choice of the virial overdensity parameter, ${ }^{4} \Delta$, which above was taken to be $\Delta=200 \rho_{\mathrm{cr}} / \bar{\rho}$. More generally, (3) can be written as $M_{\mathrm{vir}}=(4 \pi / 3) R_{\mathrm{vir}}^{3} \Delta \bar{\rho}$. The values of $\Delta$ can vary in the literature, with some common definitions being $\Delta=333$ at $z=0$ for a fiducial cosmology given by (Ade et al. 2016), which asymptotes to $\Delta=178$ at high- $z$ (Bryan and Norman 1998); or a fixed $\Delta=200$ at all redshifts, usually denoted by $M_{200 \mathrm{~m}}$.

\footnotetext{
2 The masses are indicated in terms of the solar mass $M_{\odot}$ which is equivalent to $2 \times 10^{30} \mathrm{~kg}$ in SI units. Distances are denoted in parsec $(\mathrm{pc})$, where 1 parsec corresponds to 1 arcsecond of measured parallax, and it corresponds in SI units to $3.1 \times 10^{16} \mathrm{~m}$.

3 Virial equilibrium means that it obeys the virial theorem $E_{\text {kin }}=-2 E_{\text {pot }}$ and conservation of energy. So we can describe the system only in terms of the radius $R$ and thee mass, $M$ (or velocity $V$ ) of the spherical mass concentration.

4 Not to be confused with the dimensionless power spectrum defined in (1).
} 
We identify the DM halos from numerical simulations, the N-body code $\mathrm{P}^{3} \mathrm{M}$ (Efstathiou et al. 1985), and can extract from them the abundance of halos as a function of their mass for a given redshift. The individual halos can also be analyzed in those simulation and the radial mass profile can be determined. A surprising feature encountered in those simulations is that halos appear to have a universal density profile, averaged over spherical shells. Their functional form is characterized by the Navarro, Frenk and White (NFW) profile (Navarro et al. 1997),

$$
\rho_{\mathrm{NFW}}(r)=\frac{\rho_{\mathrm{s}}}{\left(r / r_{\mathrm{s}}\right)\left(1+r / r_{\mathrm{s}}\right)^{2}} \rightarrow \begin{cases}1 / r, & \text { for } r \ll r_{\mathrm{s}} \\ 1 / r^{3}, & \text { for } r \gg r_{\mathrm{s}}\end{cases}
$$

where $r_{\mathrm{S}}$ is the radius where the slope of the profile changes and $\rho_{\mathrm{S}}=\rho\left(r_{\mathrm{s}}\right)$. We can see that this profile diverges towards the center of the halo, presenting a cusp. The amplitude of the density profile can be written in terms of $R_{200}$, as we can see from (3),

$$
\bar{\rho}=\frac{3}{4 \pi R_{200}} \int_{0}^{R_{200}} 4 \pi r^{2} \rho(r) \mathrm{d} r=3 \rho_{\mathrm{s}} \int_{0}^{1} \frac{x^{2}}{c x(1+c x)^{2}} \mathrm{~d} x
$$

where $x=r / R_{200}$, and $c:=R_{200} / r_{\mathrm{s}}$ is the concentration index and describes the shape of the distribution. With that, the NFW profile can be determined completely by $R_{200}$ (or $M_{200}$ or any other halo radius definition), and the parameter $c$. The shape of the concentration can be inferred from the same $\mathrm{P}^{3} \mathrm{M}$ simulation, where $c \propto\left(M / M^{*}\right)^{-1 / 9}(1+z)^{-1}$. We can see that, early-forming halos have a smaller radius, and they are denser than the larger ones, given the higher concentration. The NFW profile can be generalized for a three-parameter profile that better fits the DM profile of halos for all ranges in mass (Einasto 1965; Navarro et al. 2004; Gao et al. 2008).

Above we presented the spherically averaged density profiles of DM halos, described by the NFW profile. Although this presents a good fit to DM N-body simulations (that assume spherical symmetry and use shells that are distributed radially) and some observations, halos are not spherical. From halo and large cosmological simulations (Schneider et al. 2012; Jing and Suto 2002; Kasun and Evrard 2005; Bailin and Steinmetz 2005; Allgood et al. 2006), we can see, however, that the majority of the DM halos are elliptical or triaxial, with their axis aligned with the cosmic web structure. This non-spherical structure and intrinsic alignment might come at formation of the halos from the tidal field. The halo triaxiality plays a crucial hole in the interpretation of lensing data, cluster morphology and Sunyaev-Zeldovich measurements (for reviews on this topic, see (Joachimi et al. 2015; Kiessling et al. 2015), and needs to be taken into consideration.

\section{Surface brightness of galaxies}

Our capacity of observing galaxies is limited by the brightness of the sky. They can only be observed if their surface brightness, which is the brightness per area, is higher than the sky surface brightness ${ }^{5}$ where the area $A$ is the 
area of the survey $\left(\mu_{\mathrm{B}}=23 \mathrm{mag} / \operatorname{arcsec}^{2}\right)$. This can limit our understanding of the distribution of galaxies, making us miss the fainter ones. The surface brightness of a galaxy is described with respect to the radius $R$ (Binney and Tremaine 2008) as $S_{p}(R)=S_{d} \exp \left(R / R_{d}\right) \propto \exp \left(-k R^{1 / m}\right)$, where $R_{d}$ is the disk scale length, and in the second equality we have the empirical $\mathrm{S}$ érsic law. Therefore, we have the following nomenclature for the galaxies with respect to their surface brightness.

Low surface brightness (LSB) galaxies: There is no formal definition for LSB galaxies, but in general they are disk galaxies that have surface-brightness smaller than $\mu_{\mathrm{B}}$. They are believed to make the majority of the galaxies in our universe, and most of the LSB galaxies are dwarf galaxies. However, this is not necessarily the case, with LSB galaxies being galaxies in a broad range of masses, and very diverse morphologies. This low luminosity is likely associated to a small star formation rate in those galaxies. So those galaxies are believed to be DM dominated. Their rotation curves ${ }^{6}$ usually reach much smaller speeds than the ones from high surface brightness galaxies (see below), with a very slow rise before reaching the plateau region given their lower density, but broaden mass distribution.

High surface brightness (HSB) galaxies: They are usually defined as galaxies that are brighter than $\mu_{\mathrm{B}}$. They are the usual galaxies we study. The rotation curves are known to reach high velocities with a steep rise, coming from the inner region that has a higher density of baryons with narrower mass distributions than LSBs, which is described by the Newtonian baryonic acceleration. This is followed by a Kleplerian fallout to the flat part of the rotation curve.

\subsection{Discrepancies in comparison with observations}

In this section, we will show how some of the theoretical predictions from simulations of the small-scales considering the $\Lambda \mathrm{CDM}$ model compare with respect to astrophysical observations. However, this comparison is not straightforward, since we indirectly probe the dark matter inferring it from the visible matter that traces the gravitational potential of galaxies and clusters. There are a few approaches to connect the information of galaxies and the dark matter halos like forward modelling, abundance matching and kinetic measurements, and each of those methods has its difficulties and limitations. The result of this comparison is a series of discrepancies that challenges the results of the simulations, and in some cases limitations in observations.

\footnotetext{
5 The brightness of an object is a measure of the amount of light (luminosity) that we detect: $B=$ Luminosity $/ 4 \pi d^{2}$, where $d$ is the distance to the object. We use magnitude to measure the brightness of an object in a scale without units, and represented by mag.

5 A rotation curve of a galaxy shows the change in the orbital circular velocity of stars or gas clouds with respect to the distance from the center of the galaxy. An example of a rotation curves can be seen in Fig. 3 for dwarf galaxies. Different types of galaxies present very distinct rotation curves, such as low or high surface brightness galaxies. This can be seen in Fig. 14.
} 
We will present some of these challenges in this section. Some of those challenges might have complementary origin and solution, and are indeed connected, as we will discuss below.

\subsubsection{Cusp-core}

As we saw above, the expected density profile from collisionless simulations is the NFW profile which is cuspy towards the central region of the halo. Given the complex dynamics of baryonic matter in some galaxies, good laboratories to probe the halo structure are low surface brightness (LSB) galaxies and late-time dwarfs. Those systems are dominated by DM throughout their halo up until the central regions. Measuring the rotation curves of dwarf galaxies, (Flores and Primack 1994; Moore 1994) found that those measurements preferred cored isothermal profiles. Many other measurements of the rotation curves of those systems (McGaugh and de Blok 1998; Côté et al. 2000; van den Bosch and Swaters 2001; Borriello and Salucci 2001; de Blok et al. 2001a, b; Marchesini et al. 2002; Simon et al. 2005; Gentile et al. 2005, 2007; Kuzio de Naray et al. 2006, 2008) have confirmed this discrepancy, showing a constant density core with a profile with a slope $\gamma=0-5$ (considering the profile at small radius given by $\left.\rho \sim 1 / r^{\gamma}\right)$. The smallest values for this slope from dissipationless simulations are too large in comparison to the ones obtained by observations.

The recent measurement of nearby dwarf galaxies from the survey THINGS (HI Near Galaxy Survey) (Oh et al. 2011) and LITTLE THINGS (Oh et al. 2015) confirmed this discrepancy. Measuring the rotation curves from 7 to 26 nearby dwarfs, they found that the inner slope is much smaller than the NFW one $(\gamma=-1)$, with $\gamma=0.29 \pm 0.07$ for the LITTLE THINGS survey, as we can see in Fig. 3.

The situation is more complex for high surface brightness (HSB) objects given its complex inner density structure; or for galaxies with large mass, like spiral galaxies, where at small radii is dominated by baryonic matter. Even in the case of dwarf galaxies, it was pointed out that some systems present cuspy profiles, while others cored ones, presenting an unexpected diversity in the rotation curves (Oman et al. 2015). Since different results were obtained by different techniques for the same system, this shows that determining the inner slope of galaxies is a hard task.

The origin for these discrepancies can come from the fact that the simulations take into account only DM, while the properties of galaxies are also influenced by the presence of baryons. The newest hydrodynamical simulations obtained by many independent groups have shown that baryonic feedback can in fact soften the inner cusps in the profile and generate core-like profiles like the ones observed for dwarf galaxies. The main effects are supernova feedback flattening and dynamical friction from baryonic clumps (for a more detailed list of these and other baryonic processes, see (Del Popolo and Le Delliou 2017)). These simulations show a threshold mass of $M_{\text {vir }} \sim 10^{10} M_{\odot}$ below which the simulation predict profiles that are cusped (Governato et al. 2012; Munshi et al. 2013; Madau et al. 2014; Oñorbe et al. 2015; Tollet et al. 2016; Fitts et al. 2017).

However, not all simulations agree with this result. Additionally, modelling those baryonic feedback effects is challenging, and introduce many new parameters and uncertainties in modelling assumptions. Finally, not all baryonic processes that might 


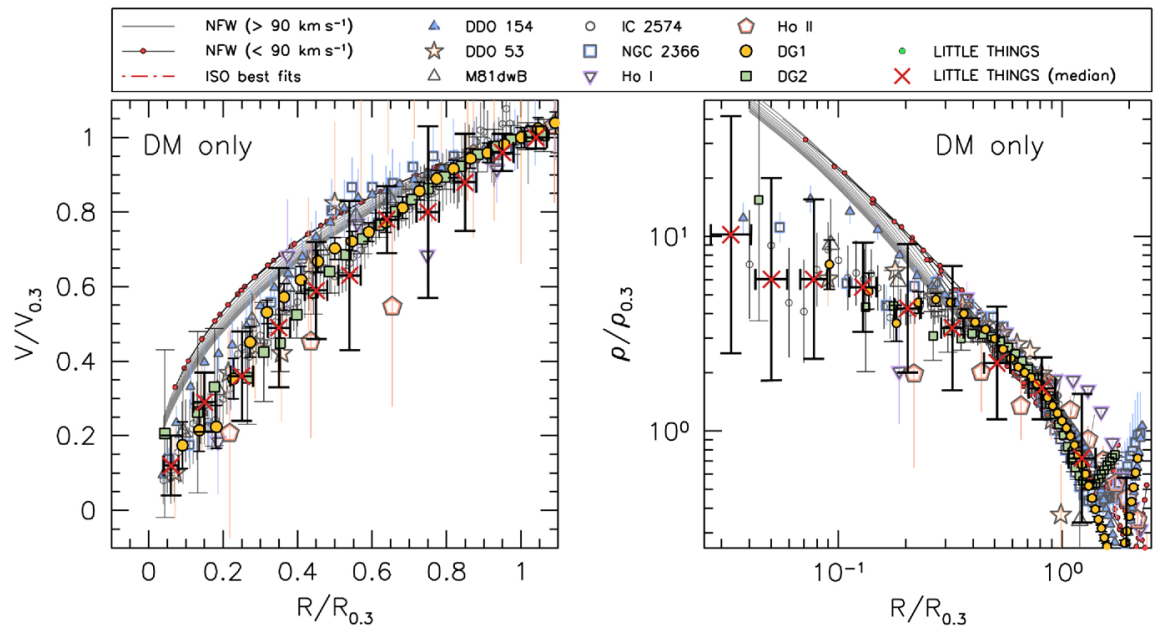

Fig. 3 Figure adapted from Oh et al. (2011), showing the results from the THINGS and LITTLE THINGS surveys. The plot shows a comparison of the velocity versus radius (rotation curve) (left panel) and density versus the radius (right panel), normalized by $R_{0.3}$, and $V_{0.3}$ and $\rho_{0.3}$, from the theoretical parametrizations of the NFW potential (solid line) and the pseudo-isothermal (dashed line), with the simulated galaxies (Governato et al. 2010), represented in the plots by the legend DG1 and DG2. In the plots observational data from 7 dwarf galaxies measured by THINGS are represented by the other points. The crosses represent the median values of the LITTLE THINGS rotation curves and density profile. We can see that the galaxies seem to follow a cored profile, while NFW predicts a cusp

influence the formation and dynamics of galaxies were included in the simulations, and that might reveal to be important for the result. It is clear that the inclusion of baryonic effects is hinting in the right direction, but until consensus is achieved, alternatives need to be considered. As mentioned before, a modification of the properties of DM might in a simple way account for that, as we will show for the case of Bose-Einstein condensate DM. An early solution to the cusp-core problem, and that explains the rotation curves with exquisite precision is a modification of the dynamics of gravity on small-scales, the MOdified Newtonian Dynamics (MOND). This is also a solution for the regularity versus diversity challenge, and its main points and shortcomings will be presented at the end of this section.

\subsubsection{Missing satellites}

Structure formation is hierarchical in nature and it is expected that the DM halos are also populated by small subhalos. This is confirmed in $\Lambda$ CDM simulations of MilkyWay-sized halos, which show that the subhalo mass function diverges toward low masses, limited only by the numerical limit. Those simulations then predict several hundreds of subhalos with $v_{\max } \sim 10-30 \mathrm{~km} / \mathrm{s}$, that are large enough to host a galaxy ( $M_{\text {peak }} \gtrsim 10^{7} M_{\odot}$ ), where $M_{\text {peak }}$ is the maximum virial mass the halos had when they formed. On the other side, until 2005 only $12 \mathrm{MW}$ classical satellites were known, with 15 more confirmed ultra-faint satellite galaxies until 2014, with the data from Sloan Digital Sky Survey (SDSS) (Drlica-Wagner et al. 2015). To date, with the inclusion of 
Dark Energy Survey (DES) data, a few more ultra-faint candidates were discovered, with the known count of satellites of more than 50. However, the number of MW galaxy satellites is still much smaller than the number predicted from simulations. This is known as the missing satellite problem, and not only appears in the MW, but also in the Local Group.

DES and future observations are expected to discover more of those ultra-faint galaxies, which can alleviate this discrepancy, but there is still a debate if this will solve the problem. Another possibility is that low-mass subhalos are there, but we just cannot see them since they have very low baryonic content. One can expect that for low mass subhalos, galaxy formation is suppressed since the photoionizing background heats the gas, reducing its cooling rate and inhibiting gas accretion for $M_{\text {vir }} \sim 10^{9} M_{\odot}$ (Efstathiou 1992; Bullock et al. 2000; Benson et al. 2002; Bovill and Ricotti 2009; Sawala et al. 2016). Star formation is also suppressed since supernova-driven winds could strip the gas out of these halos (Dekel and Silk 1986). Other mechanisms can also suppress the baryon content in the low-mass galaxies, see (Del Popolo and Le Delliou 2017), like reionization suppression. So, the visible subhalos are only a set of the entire distribution of halos that contains the non-visible faint end. It was shown recently in the hydrodynamical simulations APOSTLE (Sawala et al. 2016; Zolotov et al. 2012; Zhu et al. 2016) that apparently this mechanism can solve the difference in the number of predicted and observed satellite galaxies, thus solving the missing satellite problem. But the question remains if this process needs to be too finely tuned to solve the problem.

\section{Too big to fail}

The above mechanism that could solve the missing satellite problem leads to another challenge: the too big to fail problem. When we say that the visible subhalos of the MW are only a set representing the most massive subhalos in the total distribution of subhalos, to have agreement with $\Lambda \mathrm{CDM}$ simulations these visible MW subhalos need to correspond to the most massive subhalos predicted by the simulations. But, the most massive subhalos predicted by those simulations have central $\operatorname{masses}^{7}\left(V_{\max }>\right.$ $30 \mathrm{~km} / \mathrm{s}$ ) that are too large to host the observed satellite galaxies (Boylan-Kolchin et al. 2011, 2012), and the ones that have central mass like the expected by the MW (with $12<V_{\max }<25 \mathrm{~km} / \mathrm{s}$ ) are not the most massive ones. So, the puzzle is why should the most massive subhalos, where the gravitational potential is the strongest and the striping gas mechanisms cited above are not important, be too big to fail to form stars and galaxies? This is illustrated in Fig. 4. This problem also appears in the galaxies in the Local Group and Local Volume (Papastergis et al. 2015; Garrison-Kimmel et al. 2014), so it is not a specific property of the MW.

This higher central mass from the most massive sub-halos predicted by simulations in comparison to the MW dSphs seems to be a more general feature that appears in simulations. This discrepancy might indicate a more delicate issue related to the internal structure of the sub-halos. In this way, the too big to fail problem is more than just the problem related to the missing satellites problem, as stated above.

\footnotetext{
7 The central mass is equivalent to quoting $V_{\max }$ since $V_{\text {circ }}^{2}=G M / R$, where the maximum circular velocity is defined as the peak of the rotation curve and it is a quantity less affected by tidal stripping (Penarrubia et al. 2008).
} 


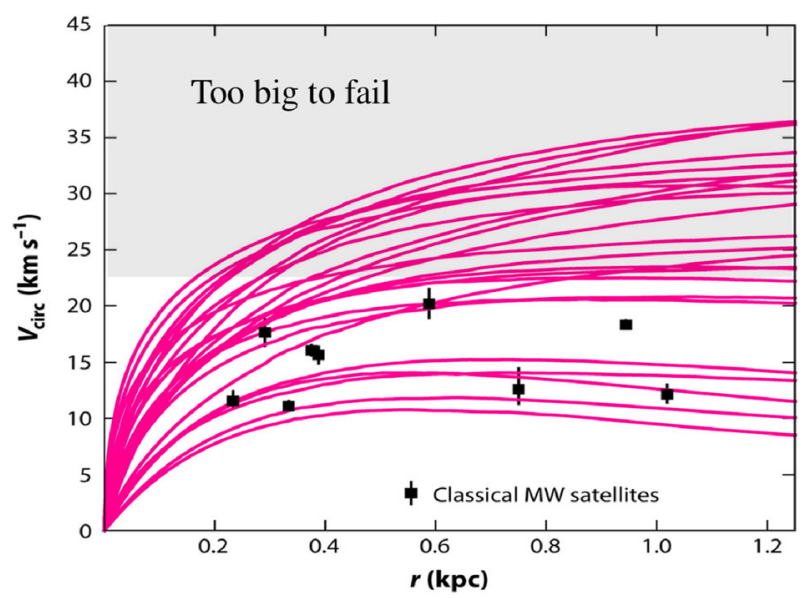

Fig. 4 Figure adapted from Bullock and Boylan-Kolchin (2017), showing the circular velocity versus the radius of specific subhalos from the Aquarius simulation that have $V_{\max }>30 \mathrm{~km} / \mathrm{s}$ (magenta lines). Those are known to have halos that are very massive and expected to host the formation of starts. However, as we can see from the data points corresponding to classical MW satellites with masses $M \sim 10^{5}-10^{7} M_{\odot}$, in the most massive of those subhalos, with $M>10^{8} M_{\odot}$ shown in the gray region of the plot, we do not observe satellites. This means that the more massive subhalos predicted by the simulations are too big to fail to form stars and galaxies

Like for the other problems, it was proposed that some astrophysical processes driven by baryons could be important on those scales and solve the too big to fail problem. However, these solutions seem to only work for the MW and for very efficient feedback, like the supernova feedback that only solves the too big to fail problem if very efficient. This is an intense topic of debate and no consensus appears to have been reached. As these notes were being written, there has been claims that the too big to fail problem has been solved (Ostriker et al. 2019).

As for the cusp core problem, different DM physics could solve those problems by having a mechanism that suppresses the formation of small-scale subhalos, and that reduces the central densities of massive subhalos (or modifies the dynamics of the central regions). We are going to show how the models with Bose-Einstein condensation address some of those problems.

\subsubsection{Diversity vs. regularity: scaling relations}

Although our universe came from very smooth initial conditions, nowadays the diversity of galaxies that we find in the universe is extraordinary. This incredible diversity of galaxies, though, presents a surprising regularity. This fact is manifest in several scaling relations that are shown to hold very tightly for a diverse range of galaxies. These relations relate the dynamical and baryonic properties of galaxies, and hold even for DM-dominated systems, and they are one of the most tantalizing aspects of galaxy phenomenology, representing the most pressing challenge for $\Lambda \mathrm{CDM}$ on small-scales. 

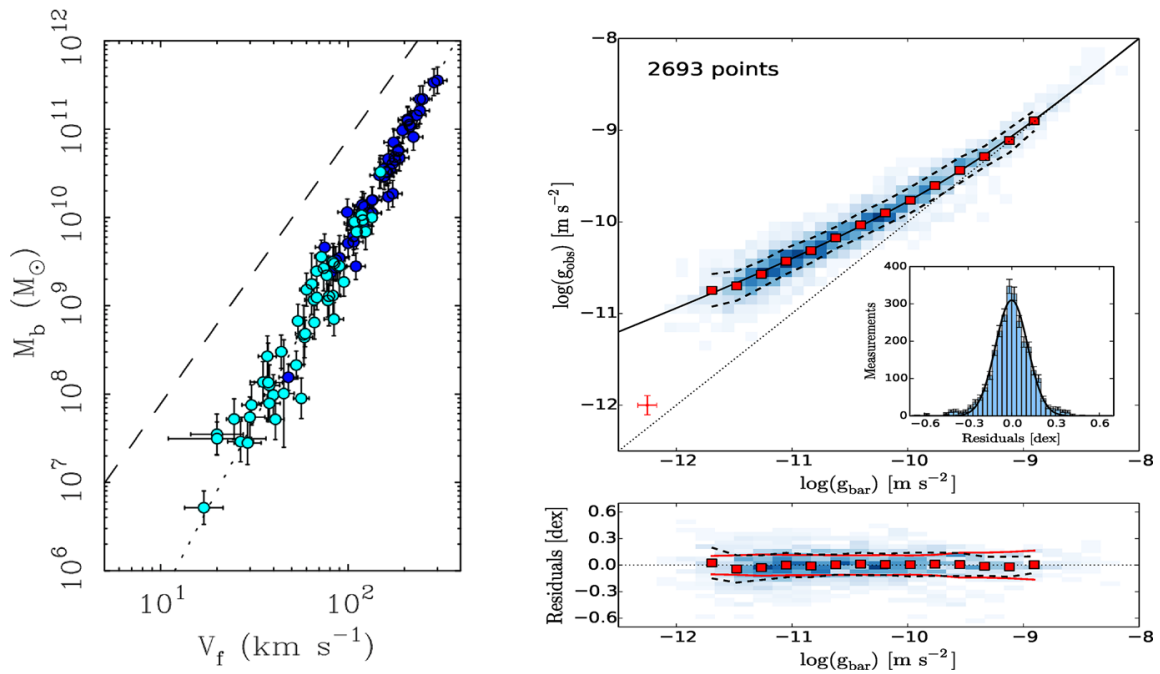

Fig. 5 Left panel: the Baryonic Tully-Fisher Relation (BTFR), which shows the relation between the baryonic total mass $\left(M_{\mathrm{b}}\right)$ and the asymptotic circular velocity. Dark and light blue points represent star and gas dominated stars, respectively. The dashed line represents the relation expected for $\Lambda \mathrm{CDM}$, with slope equal to 3 ; while the dotted line which better fits the data, has slope 4 . Image reproduced with permission from from Famaey and McGaugh (2012), copyright by the authors. Right panel: Plot of the Radial Acceleration Relation for 153 SPARC galaxies. The fit to the data is given by the solid line while the dotted line is the unit line. The insert is a histogram of the residuals. The red uncertainty bars represent the uncertainty in each individual point. The lower panel shows the residuals, and the red uncertainty bar shows the mean uncertainty on individual points. The dashed lines represent the rms value in each bin and the solid red lines represent the observational uncertainties and variation between the stellar mass-to-light-ratio from galaxies. Image reproduced with permission from McGaugh et al. (2016), copyright by APS

The most famous of those relations is the Baryonic Tully-Fisher relation (BTFR) (McGaugh 2005, 2008), which relates the total baryon mass (including stars and gas) of the galaxy to the asymptotic circular velocity in galaxies, $V_{\mathrm{f}}$ (this is the velocity measured at the flat portion of rotation curves):

$$
V_{\mathrm{f}}^{4}=a_{0} G M_{\mathrm{b}},
$$

where $a_{0}$ is the critical acceleration, a scale that appears in observations. Its value can be obtained from the data and given by $a_{0} \sim 1.2 \times 10^{-8} \mathrm{~cm} / \mathrm{s}$. The BTFR expands the regime of validity of the Tully-Fisher relation which relates the luminosity, instead of the total mass, to the circular velocity. Luminosity is a probe of the stellar mass, and in the BTFR, the observed gas mass is also considered on top of the stellar mass. This extends the validity of the scaling relation by many decades in mass. This empirical scaling relation is shown to hold for large ranges of masses, six generations, with a very small scatter, compatible to the size of the error bars. The left panel of Fig. 5 presents the BTFR. As we can see, the slope of the BTFR is different from the one predicted by $\Lambda \mathrm{CDM}, V_{\mathrm{f}}^{3} \propto M_{\mathrm{b}}$, shown by the dashed line.

There is another general scaling relation that also displays the interesting behaviour of galaxies: the mass discrepancy acceleration relation (MDAR). This is more general 
since the BTFR can be obtained from the MDAR at large distances in the disk. The MDAR is a relation between the gravitational acceleration from baryons alone $\left(g_{\text {bar }}\right)$, from the distribution of gas and stars in galaxies (McGaugh et al. 2016; Lelli et al. $2017)$, and acceleration inferred from rotation curves $\left(g_{\text {obs }}=V^{2} / r\right)$. As it can be seen in the right panel of Fig. 5, this scaling relation shows a remarkably tight correlation between these quantities for very diverse and large number of galaxies This can be seen by comparing the interval determined by the solid red lines and the uncertainty in each individual point, represented by the red uncertainty bars on the top figure, with the dashed lines show that the data is compatible with negligible scatter.

This relation shows us that in regions of high acceleration, where $g_{\mathrm{obs}}>a_{0}$ and baryons dominate, one has $g_{\text {obs }} \sim g_{\text {bar }}$. For low accelerations, in the central regions where it is expected to be DM dominated, this relation deviates from the unit line. This suggests a very curious behaviour: the baryon mass distribution dictates the behaviour of the rotation curve at all radii, even for the regions expected to have less baryons. And this behaviour holds even for galaxies that are DM dominated.

These empirical relations, coming directly from observations, show the surprising feature that in galaxies the dynamics is dictated by the baryon content, even when DM dominates. Even more unexpected these relations are very tight, showing very little spread, even if they come from very diverse types of galaxies. As pointed out in Bullock and Boylan-Kolchin (2017), what dictates the dynamics in these correlations is the baryon mass, which is the sum of gas and stars, and not only the stellar mass, which is the one that is expected to correlate more with the total feedback energy.

Recently, it was shown by many groups that these relations can be explained within the $\Lambda$ CDM paradigm (Navarro et al. 2017; Ferrero et al. 2017; Garaldi et al. 2018; Dutton et al. 2019; Navarro 2019) using the latest hydrodynamical simulations like EAGLE (Schaye et al. 2015; Crain et al. 2015), APOSTLE (Sawala et al. 2016), Illustris (Illustris 2014), ZOMG (Borzyszkowski et al. 2017; Romano-Diaz et al. 2017; Garaldi et al. 2017), and NIHAO (Wang et al. 2015). Those simulations include several baryonic effects (like star formation, stellar evolution, metal enrichment, gas cooling/heating, galactic outflows and $\mathrm{BH}$ feedback, among others) to their $\Lambda \mathrm{CDM}$ simulations. ${ }^{8}$ Those new large volume and high-resolution simulations, like Illustris and EAGLE, have also been able to reproduce the features of the rotation curves of galaxies within $\Lambda \mathrm{CDM}$. This large amount of progress in the simulation side is very encouraging.

However, some questions still remain. While the BTFR and the MDAR trends can indeed be reproduced by those simulations, it is pointed out by most of the authors that the scatter obtained in the scaling relations is larger than the one expected from data (some authors claim that this spread is correlated with the errors in the stellar feedback). The question remains though whether this is a matter of improving the feedback models and/or resolution of the simulation, or if given the stochastic nature of the feedback effects, they will ever be able to give such tight correlations. Another point that is important to be answered is about the importance of these baryonic feedbacks since these groups do not agree on how very sensitive to the feedback model the simulations

\footnotetext{
8 This review will not enter into the details of such baryonic effects that are taken into account in those simulations. This is a field of its own, very rich and fast developing, and discussing those effects is not the scope of this review.
} 
are, which is intriguing. The way those effects are introduced in the simulations is by parametrizing their effects, instead of introducing all of these feedback mechanisms from first principles, which is understandable given the complexity of each of those phenomena. This includes many new parameters to the simulations. And different simulations might use different parametrizations. Those simulations also still do not go all the way until dwarf galaxies, ${ }^{9}$ which are DM dominated and where most of the tension is. In summary, this is a very challenging and exciting field and a lot of progress has been done on the simulation side with results that are very encouraging to explain the formation and dynamics of galaxies. But there are some uncertainties in those results and the simulations still do not fully reproduce the observations.

\subsubsection{What the small-scales tell us}

As we saw above, the small-scales hold precious information that can help us understand astrophysical processes, or even the nature of DM. This is revealed by the challenges presented above, which show rich dynamics on galactic and sub-galactic scales. There are a number of ways that these discrepancies can be addressed. Within $\Lambda \mathrm{CDM}$, this can done by including baryonic effects, which as we saw in the previous sections seem to address partially or completely some of those puzzles. Another proposal for solving some of the puzzles of galactic evolution is more radical and proposes a universe without DM that has a modified force law for small accelerations, the MOdified Newtonian Dynamics (MOND). See box below for a discussion of MOND.

A third avenue is to modify the DM paradigm. Different models of dark matter can affect the formation of structures in distinct ways, both in the linear and in the non-linear regimes. Therefore, the small-scales offer an opportunity to probe the microphysics of DM, beyond the hydrodynamical large-scale CDM paradigm. The non-linear regime can be specially changed by modifications of this paradigm, as we can see in Fig. 2. This regime can be probed using galaxies, and for even smaller scales satellite galaxies and studying substructures. This could help find new properties of DM, that could help elucidate its nature.

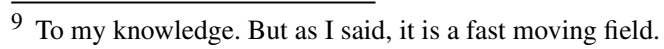




\section{MOND empirical law -}

Milgrom, in Milgrom 1983a; Milgrom 1983b; Milgrom 1983c, motivated by the scaling relations and rotation curves of galaxies, made a remarkable observation about the mass discrepancy in galaxies. He observed that the mass discrepancy can be determined by the observed baryonic matter, and can be described by the simple empirical law,

$$
a= \begin{cases}a_{\mathrm{N}, \mathrm{b}}, & \text { for } a_{\mathrm{N}, \mathrm{b}} \gg a_{0}, \\ \sqrt{a_{0} a_{\mathrm{N}, \mathrm{b}}}, & \text { for } a_{\mathrm{N}, \mathrm{b}} \ll a_{0},\end{cases}
$$

where $a_{\mathrm{N}, \mathrm{b}}=G M_{\mathrm{b}}(r) / r^{2}$ is the Newtonian acceleration due to baryons. The scale $a_{0}$ appears naturally from observations, like we saw in the previous subsection, and its value can be fitted by the data ${ }^{10}$ giving $a_{0} \sim 1.2 \times 10^{-8} \mathrm{~cm} / \mathrm{s}^{2}$. This scale separates the regimes where the centripetal acceleration experienced by a particle is given purely in terms of the Newtonian (baryonic) acceleration at large acceleration, and at small acceleration, by the geometric mean of $a_{\mathrm{N}, \mathrm{b}}$ and $a_{0}$.

The relation works very well fitting the rotation curves of galaxies, both HSB and LSB galaxies. LSB galaxies (which were predicted by Milgrom), are DM dominated, or in the language of MOND, have low accelerations, given their low stellar surface density. It is also remarkably successful in explaining the empirical scaling relations (for a review see Sanders and McGaugh 2002; Broeils 1992; Begeman et al. 1991; Famaey and McGaugh 2012).

More importantly, this empirical relation reveals a very interesting and curious fact. It seems that the dynamics in galaxies is driven by the baryons, even for galaxies that are DM dominated. This seems to indicate a long range correlation between baryons on galaxies.

This fact made Milgrom think that a fifth force was responsible for this correlation, instead of DM, and that this relation could come from a modification of gravity at those scales. In order to try to get the empirical law (7) as a modified gravity theory, (Bekenstein and Milgrom 1984) described an effective theory for MOND, which we will call full MOND. This can be accomplished by having a scalar field coupled to gravity with effective Lagrangian, 


$$
\mathcal{L}_{\mathrm{MOND}}=-\frac{2 M_{\mathrm{pl}}^{2}}{3 a_{0}}\left[(\partial \phi)^{2}\right]^{3 / 2}+\frac{\phi}{M_{\mathrm{pl}}} \rho_{\mathrm{b}}
$$

which represents a scalar field with a non-canonical kinetic term that is conformally coupled to matter. This Lagrangian, for static and spherically symmetric source, results in a modified Poisson equation

$$
\nabla \cdot\left(\frac{|\nabla \phi|}{a_{0}} \nabla \phi\right)=4 \pi G \rho, \quad \phi^{\prime}=\sqrt{a_{0} \frac{G M(r)}{r^{2}}}=\sqrt{a_{0} a_{\mathrm{N}, \mathrm{b}}}
$$

where in the second equation the spherical symmetry was assumed with " $"$ ", denoting derivative with respect to the radial coordinate. This theory describes that on top of the Newtonian force, there is a scalar field mediated force, which is given by the MONDian acceleration. This is a simplified version of their theory, since in their theory they have a way of making an interpolation between the different regimes. This theory also has a fractional power kinetic term, which might be problematic.

With the current precise observations on large scales, specially from the CMB anisotropies and lensing observations, any theory that does not have DM is not compelling. Indeed, this is a problem for theories without DM, in particular MOND, since the measurement of the third peak of the CMB anisotropies (Skordis et al. 2006; Skordis 2009). This full MOND theory cannot explain galaxy clusters, since it does not predict an isothermal profile. Many attempts were made to extend MOND, by including DM, to try to explain the observation on scales larger than galactic, or extending it to relativistic regimes (see reviews cited above).

However, the empirical relation (7) is incredibly successful. That alone, without the assumptions of full MOND (no DM and modified gravity), even in the context of $\Lambda \mathrm{CDM}$, is a powerful statement about how DM is distributed in galaxies: in regions where baryons dominate, the theory behaves like Newtonian theory, and in regions where the DM dominates, the DM mass is uniquely determined by the baryonic distribution, $G M(r) / r=\sqrt{G M_{\mathrm{b}}(r) a_{0}}$.

Given the shortcomings of the full MOND, but the great successes of the empirical law, instead of trying to obtain this theory from a fundamental Lorentz invariant theory, the idea is to obtain the MOND dynamics from a theory of DM. In this way, MOND dynamics emerges only at galactic scales while maintaining the CDM behaviour on large scales. This is achieved in the theory of DM Superfluid that will be presented in Sect. 4.

\footnotetext{
10 A funny numerical coincidence is that the measured scale $a_{0}$ is related to the Hubble parameter today, $a_{0} \approx c H_{0} / 2 \pi$, which is natural units yields $a_{0} \sim H_{0}$. Does this indicate something?
} 
The goal of this section was twofold. First, we wanted to give an overview of the socalled small-scale problems of the CDM, which was the motivation for some ULDM models to be proposed. We wanted to introduce the problems in a way that the reader can understand why the mechanisms proposed by the models in Sect. 4 address and solve each of those small-scale controversies. For example, we are going to see that the FDM model has to have a certain range of mass to solve the cusp-core problem and the satellite problem; or that the DM superfluid model has a modified dynamics of small-scales, reproducing the MOND behaviour, which explains the rotation curves of galaxies and the scaling relations.

The second goal, and perhaps the most important was to give an brief overview of the rich astrophysics that takes place in galaxies, introducing important concepts and observations available on these scales. This is important since the main feature of the ULDM models is to present a new phenomenology on small-scales coming from the non-CDM behaviour of ULDM. In this way, the small-scale observables offer an important window to test the nature of DM.

\section{Bose-Einstein Condensation and Superfluidity}

In this section, we present a short review of Bose-Einstein condensation (BEC) and superfluidity. The goal of this section is to give an introduction to the basic theory, properties and the methods used to describe those systems, so they can be applied to the case of DM in the next section. The different description of those systems and their limit of validity are very important to be able to understand the construction and validity of the DM models presented next and why they present different phenomenologies and astrophysical consequences.

Bose-Einstein condensation is one of the most fascinating phenomenon of quantum mechanics. Since it was theorized in the year of 1920s, by Satyendra Nath Bose and Albert Einstein, its experimental realization opened the door for many advances in the physics of many-body systems, and even to the application of this phenomenon in other fields like cosmology. Its first experimental realization was done in 1995 by two independent groups using laser and magnetic cooling device to cool down rubidium atoms gas (Anderson et al. 1995; Davis et al. 1995). Nowadays, BECs are observed in helium, ultra-cold atomic gases, quasi-particles in solids, multi-component (mixtures) of BECs, among other systems.

Following on the works of Bose (1924), which described the quantum statistical properties of photons, Einstein extended this concept to a gas of non-interacting particles of integer spin, later called bosons as a tribute to Bose, that follows a Bose-Einstein statistics (Schay 1924). This Bose gas has the property that at low temperatures a large number of these bosons, described then as quantum oscillators, condense into the lowest momentum state, exhibiting long range coherence. This physical phenomenon initiated the idea of Bose-Einstein condensation.

A BEC is defined as a system where at very low temperatures a large fraction of the bosons of the system occupy the lowest energy state of a configuration. This macroscopic occupancy of the ground state is an inherently quantum mechanical phenomenon. Physically we can interpret it as a consequence of the wavelike nature 
of these particles at low temperatures, where the de Broglie wavelength of these bosons is larger than the inter-particle separation, and their wavepackets superpose and form a coherent macroscopic wavefunction describing the entire system. The BEC is then described by a single wavefunction of the system, linking to the long range coherence property of a condensate.

A few years after BEC was theorized by Einstein, another intriguing macroscopic quantum mechanical phenomenon was discovered: superfluidity. In 1937, Kapitza (1938) and independently Allen and Misener (1938), conducting experiments with helium-4 realized that after cooling down this liquid to a certain temperature, the fluid starts flowing without friction, even climbing the walls of the container where it was stored. Fluids that exhibit this behaviour, characterized by a zero viscosity, are called superfluids. Landau provided a phenomenological description of this effect which rendered him the Nobel prize in 1962. It was proposed by London (1938), after the development of laser cooling techniques for atomic gases, that the properties of $\mathrm{He}^{4}$ superfluid are related to BEC. This was not obvious given that the (textbook) description of BEC as an ideal non-interacting Bose gas, contrary to ${ }^{4} \mathrm{He}$ that is a strongly interacting fluid. This gave relevance to the, until then, only theoretical ideas of Einstein, and BEC became a rich topic of research. The relation between superfluids and BECs was confirmed years later in ultracold atomic gases where almost the entire fluid at low temperatures is condensed and exhibits superfluidity.

It is very challenging to describe the strongly interacting helium system. A weakly interacting Bose gas was then proposed by Bogoliubov, as a modification of the noninteracting Bose gas model, to study the Bose-Einstein condensation and superfluidity. In this way, superfluids can be modelled by a Bose-Einstein condensate that has selfinteraction, and superfluidity is described as being achieved through interactions in a BEC. Notice that BEC can happen even in the absence of self-interaction, as seen above, since it is a statistical property of a gas of bosons in low temperature, but this system does not exhibit superfluidity. The weakly interacting theory is used to describe many superfluid systems at certain limits. This description, tough, evolved in the last few years to extend and generalize this framework to finite temperature systems, mixtures and even stronger interacting system corrections. New frameworks also emerged to describe different systems that cannot be modelled by the weaklyinteracting theory. One of those ideas based on the hydrodynamical description is to write these systems as an effective field theory (EFT) order to describe the system macroscopically using symmetry alone without the need of working its microscopic description. This EFT, depending on the symmetries of the system, can recover at some limits the weakly interacting superfluids, but also can be used to describe more general superfluids, superconductors and even systems like the unitary Fermi gas (Kevrekidis et al. 2001; Baker 1999), which is a gas of fermions that interact through a strong two-body coupling that is a superfluid in the ground state.

The theoretical description of those condensed matter systems, together with the experimental efforts, is a field of research that is in fast development. In this review we are going to describe the basic concepts on BEC and superfluid, and detail the different descriptions and properties of these systems. We start by describing the noninteracting ideal gas, where condensation was first conceptualized to present in more detail the definition and the conditions for condensation. We then start to describe 
superfluidity. We show first the definition of superfluidity as defined in Landau's theory of superfluidity. We then describe a more concrete model for a BEC where superfluidity is present, the weakly interacting Bose gas. This is the simplest example of superfluidity. We show how this model describes condensation and superfluidity. We then follow to show the field theory description of the superfluid, where the system is described as a system which undergoes spontaneous symmetry breaking caused by the condensate. This description brings advantages and makes clear the study of many features in BECs and superfluids. As a low-energy description of the superfluid, we present the EFT of superfluids as another description of more general superfluid systems. We finalize describing what happens when we rotate a superfluid, showing the nucleation of vortices upon rotation.

Not linked to what we will discuss in the review, but an important fact. Nowadays, it is known that superfluidity is not necessarily linked to condensation. Recent investigations seem to point that there are states where you can have superfluidity for the majority or all the particles in the system, while only a small fraction is condensed. This happens for example for liquid helium below a certain temperature.

\subsection{Non-interacting ideal gas}

We start our discussion with the non-interacting Bose gas. The properties of this system are a consequence purely from the quantum statistic of indistinguishable bosons. We will see that in the grand canonical ensemble we can write the Bose distribution function in which we can see the conditions for Bose-Einstein condensation.

We want to describe here a theoretical gas of many non-interacting bosons in a box. In a system with a large number of particles $(N)$, it is impractical to try to determine the state of each particle or even the collective many-body wavefunction that describes this system $\Psi\left(\mathbf{r}_{1}, \ldots, \mathbf{r}_{N}, t\right)$. In this sense, to describe a system with many particles that can occupy many different states, we represent the system using a statistical ensemble description. To describe the state of this collective system, one does not need to label the state of each particle, but to determine the number of particles in each state of the system. The ensemble that is specially convenient for this task of deriving the probability of microscopic states is the grand canonical ensemble (GCE). Since our system is composed of bosons, which are indistinguishable particles $\Psi\left(\mathbf{r}_{1}, \mathbf{r}_{2}, \ldots, \mathbf{r}_{N}, t\right)=\Psi\left(\mathbf{r}_{2}, \mathbf{r}_{1}, \ldots, \mathbf{r}_{N}, t\right)$, called Bose symmetry, this ensemble is useful to describe the system where many particles can occupy the same state.

The GCE is a statistical ensemble that describes a system that is in contact in thermal and chemical equilibrium with a large reservoir, in a way that there is exchange of energy and particles with the reservoir. This exchange of particles with the reservoir makes the number of particles in the system to fluctuate, although the number of particle of the system plus reservoir is constant. As the system is in equilibrium, the energy and particle number fluctuate around an average. This ensemble can be described by the following constants: the chemical potential $(\mu)$ and the temperature $(T$ ), which hold for a system (of volume $V$ ). If the GCE is applied to small systems, an additional condition is necessary: that the gas is diluted. In principle, the probability of finding the system in a state $s$ with energy $\epsilon_{s}$ and $n_{s}$ particles, or occupation number 
$n_{s}$, is given by

$$
P_{\mathrm{s}}=\frac{1}{Z_{s}^{\mathrm{GC}}} e^{\beta\left(\mu n_{s}-\epsilon_{s}\right)}, \quad \text { with } \quad Z_{s}^{\mathrm{GC}}=\sum_{s} e^{\beta\left(\mu n_{s}-\epsilon_{s}\right)},
$$

where $\beta=1 /\left(k_{\mathrm{B}} T\right)$, with $k_{\mathrm{B}}$ the Boltzmann constant. The chemical potential $\mu=$ $(\partial E / \partial N)_{S, V}$ is the energy required to add one particle to the isolated system, fully determined by $N$, the total number of particles, and $T$. The chemical potential is defined to be negative (so no unphysical negative occupation occurs). The total energy of the system is given by $E=\sum_{s} n_{s} \epsilon_{s}$. The normalization $Z_{s}^{\mathrm{GC}}$ is the grand canonical partition function.

With the GC distribution function, we can then evaluate the average occupation number,

$$
\left\langle n_{s}\right\rangle=\sum_{n_{s}} n_{s} P_{s}=\frac{1}{e^{\beta\left(\epsilon_{s}-\mu\right)}-1},
$$

where the sum converges for $\mu<\epsilon_{s}$. This is the Bose-Einstein distribution. This gives us the total number of particles in the system:

$$
N=\sum_{s} n_{s}=\sum_{s} \frac{1}{e^{\beta\left(\epsilon_{s}-\mu\right)}-1}
$$

We can separate the total number of particles into two contributions,

$$
N=N_{0}+N_{\mathrm{T}}
$$

where $N_{0}=1 / e^{\beta\left(\epsilon_{0}-\mu\right)}-1$ is the number of particles with $\mathrm{s}=0$, which is the number of particles in the condensate, with $\epsilon_{0}$ indicating the lowest energy of the single particle state. The number of particles that are not in the ground state, not in the condensate, also called the thermal component of the gas, $N_{\mathrm{T}}=\sum_{s \neq 0} n_{s}$. We can replace the sum for an integral and, from the partition function, the thermal component is given by

$$
N_{\mathrm{T}}=\frac{V}{\lambda_{\mathrm{T}}} \int_{0}^{\infty} \mathrm{d} \epsilon \frac{\epsilon^{1 / 2}}{e^{\beta(\epsilon-\mu)}},
$$

where $\lambda_{\mathrm{T}}=\sqrt{2 \pi \hbar^{2} \beta}$. For a fixed temperature, $N_{\mathrm{T}}$ reaches a maximum when $\mu=\epsilon_{0}$. So, $N_{\mathrm{T}}$ is limited, meaning that in this limit there is a finite number of particles not in the ground state. At this same point in this limit, $N_{0}$ can diverge showing that the number of particles in the ground state grows becoming macroscopically occupied. This macroscopic occupation of the ground state is seen as a condensation and this phenomenon is called Bose-Einstein condensation.

The critical temperature $T_{\mathrm{c}}$ defines the temperature below which there is the formation of the BEC. We can define it as the temperature above which all the particles of the system are not going to be in the condensate: $N_{\mathrm{T}}\left(T_{\mathrm{c}}, \mu=\epsilon_{0}\right)=N$. The chemical 
potential can be zero at $T_{\mathrm{c}}$, and from (14), for the maximum $\mu=\epsilon_{0}=0$, we can get that

$$
T_{\mathrm{c}}=\left(\frac{2 \pi \hbar^{2}}{m k_{\mathrm{B}}}\right)\left(\frac{n}{\zeta(3 / 2)}\right)^{2 / 3}
$$

where $n$ is the total number density and $\zeta(3 / 2) \approx 2.612$ is the Riemann's zeta function. With that, for $T<T_{\mathrm{c}}$, we expect that most of the particles are going to be in the condensate, and the number of particles in the condensate is

$$
N_{0} / N=1-\left(\frac{T}{T_{\mathrm{c}}}\right)^{3 / 2} .
$$

Complementary, the number of particle in the thermal component is $N_{\mathrm{T}}=$ $N\left(T / T_{\mathrm{c}}\right)^{3 / 2}$. From that expression we can see that the occupation becomes macroscopic towards small temperatures, as we can see in Fig. 6 . This indicates the formation of a BEC. This condition that a BEC can form for $T<T_{\mathrm{c}}$ can be translated into the condition: $n \lambda_{\mathrm{dB}}^{3} \gg 1$, where $\lambda_{\mathrm{dB}}=\sqrt{2 \pi \hbar^{2} /\left(m k_{\mathrm{B}} T\right)}$ is the thermal de Broglie wavelength that gives the coherence length of the gas. This condition indicates that the gas needs to be dilute for condensation to happen. This condition is also equivalent to having the de Broglie wavelength of the particles overlap and the system being described by a macroscopic wave-function. When $T=0$, all the particles of the system will be in the ground state and the condensate is described by a single macroscopic wavefunction. As we see from Fig. 6, at high temperatures, condensation is broken and the system behaves as a gas of individual massive particles.

The de Broglie wavelength $\lambda_{\mathrm{dB}}$ : $\quad$ associated wavelength of a massive particle given by $\lambda_{\mathrm{dB}}=h / p=h / m v$, where $p$ and $v$ are the momentum and velocity of the particle with mass $m$, respectively. For an ideal gas of temperature $T$ in a volume $V$, we have the thermal de Broglie wavelength which determines the coherent length of the gas. The thermal de Broglie wavelength can be defined then, where the characteristic thermal momentum is $p_{\mathrm{T}}=\sqrt{2 \pi m k_{\mathrm{B}} T}$, by:

$$
\lambda_{\mathrm{dB}}=\sqrt{\frac{2 \pi \hbar^{2}}{m k_{\mathrm{B}} T}} .
$$

When the thermal de Broglie wavelength is much smaller than the interparticle distance $(d)$, we have a gas of free particles. Otherwise, we have condensation as studied here.

In summary, Bose-Einstein condensation can happen for ideal gases. The condition for condensation is that the occupation number of the ground state is so large that becomes macroscopic when $T<T_{\mathrm{c}}$. This can be translated in a condition for 


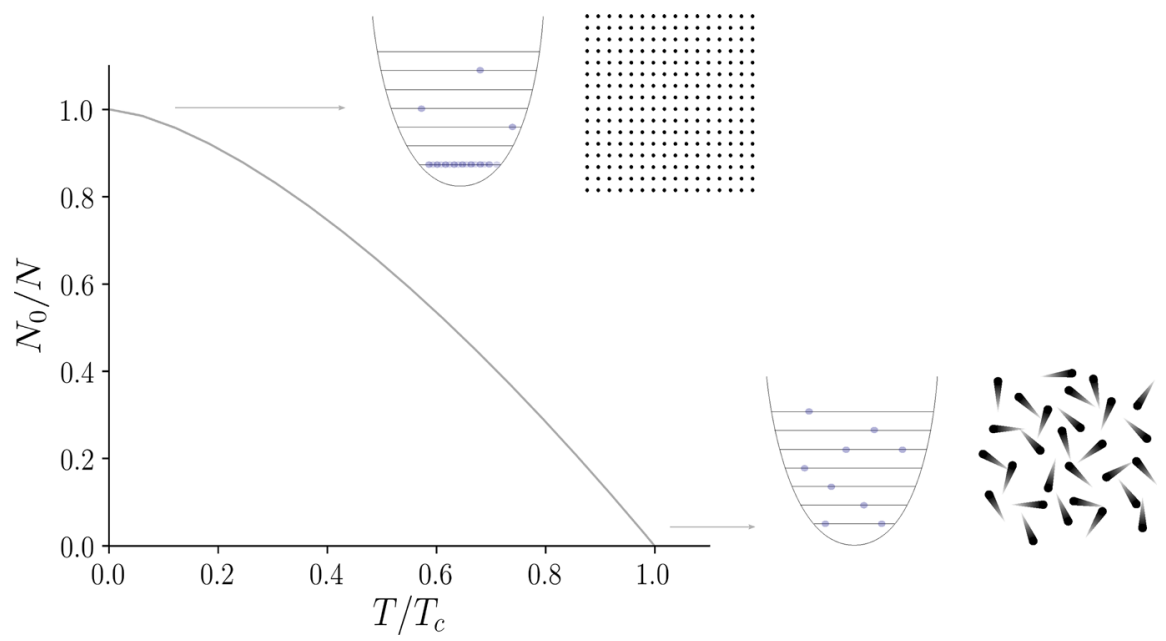

Fig. 6 In this figure, we plot the number of particles in the ground state, normalized by the total number of particles, with respect to the temperature for the non-interacting Bose gas. We show schematically that for higher temperatures $T \geq T_{\mathrm{c}}$ the system is in the normal state where the particles behave as free particles and occupy all energy levels. As the temperature is lowered, when $T<T_{\mathrm{c}}$ we have the formation of a condensate described by a macroscopic wavefunction. When $T=0$, all particles of the system are in the ground state and we have a pure BEC, described by a single wavefunction

condensation: if $n \lambda_{\mathrm{dB}}^{3} \gg 1$, there is the formation of a BEC. With that we can see that this very simple theoretical model already shows this intriguing macroscopic quantum phenomenon that would be confirmed experimentally many years later.

Condition for condensation of a non-interacting ideal gas

The condition for condensation of an ideal gas of $N$ bosons in thermal equilibrium with volume $V$ and temperature $T$ is:

$$
T<T_{\mathrm{c}} \Longleftrightarrow n \lambda_{\mathrm{dB}} \gg 1 \Longleftrightarrow \lambda_{\mathrm{dB}} \gg d \sim\left(\frac{V}{N}\right)^{1 / 3}=\left(\frac{1}{n}\right)^{1 / 3}
$$

These conditions state that the temperature must be smaller than the critical temperature; or that we have a macroscopic occupation number of the ground state $N_{0}$; or that the de Broglie wavelength needs to be bigger than the mean space between particles in order to have quantum degeneracy. It is easy to see that these conditions are equivalent.

\subsection{Landau's superfluid model and criteria for superfluidity}

A few years after BEC was theorized, another striking macroscopic quantum phenomenon was observed, superfluidity. Landau constructed a phenomenological theory 
to explain the results of superfluidity in helium, which was observed to flow in thin capillaries. This phenomenological theory, however, is quite general to describe superfluids and gives general conditions for the appearance of superfluidity.

This theory has the goal of explaining why in superfluids charge is transported without friction. As we described above, according to London's ideas, to have superfluidity, one needs to have a BEC. The condensate has the role of transporting charge. So we consider a superfluid as the condensate that transports charge without losing energy. Dissipation of the condensate, which is equivalent to friction in the fluid, is caused by exciting particles out of the condensate. We have a superfluid in the limit of no or low dissipation, and the superfluid is lost in the limit of high dissipation. We present now the conditions for that to happen.

Consider a superfluid moving through a capillary with velocity $v_{\mathrm{s}}$. The energy of elementary excitations is given by Pitaevskii and Stringari (2016),

$$
E=E_{\mathrm{kin}}+\epsilon_{p}+\mathbf{p} \cdot \mathbf{v}_{\mathrm{s}}
$$

in the rest frame of the capillary. The kinetic energy of the fluid is given by $E_{\text {kin }}$, and $\epsilon_{p}>0$ and $\mathbf{p}$ are the energy of the excitation and momentum in the frame of the fluid, and translated to the frame of the capillary. Dissipation happens when $\epsilon_{p}+\mathbf{p} \cdot \mathbf{v}_{\mathrm{s}}<0$. This can only be negative if its minimum, when $\epsilon_{p}+p v_{\mathrm{s}} \cos (\theta)$ where $\theta=n \pi$ for $n$ integers, is smaller than zero: $\epsilon_{p}-p v_{\mathrm{s}}<0$. With that we can determine the critical velocity:

$$
v_{\mathrm{c}}=\min _{p} \frac{\epsilon_{p}}{p}
$$

For $v_{\mathrm{s}}<v_{\mathrm{c}}$, with $v_{\mathrm{c}} \neq 0$, the system transports charge without dissipation and the coherence of the BEC is maintained. This is the first criteria for superfluidity. The second necessary criteria is that $v_{\mathrm{c}}$ cannot be zero, so we need to have a condensate that transports the charge. A non-interacting (pure) Bose gas like we saw in the previous section has $v_{\mathrm{c}}=0$, so it cannot be a superfluid. A weakly interacting Bose gas has $v_{\mathrm{c}} \neq 0$ and it is a good representation of a superfluid.

As we are going to see in the next section in the case of the weakly interacting BEC because of the spontaneous breaking of U(1) symmetry, a Goldstone mode appears, the phonon. This mode is gapless $\epsilon_{p=0}=0$ and it is an elementary excitation of the superfluid. Even for that mode, the critical velocity is not zero, so there is some cost for producing the gapless excitation. For this weakly interacting Bose gas, the critical velocity is the fluid sound speed, and Landau's criteria for superfluidity becomes

$$
v_{\mathrm{s}}<v_{\mathrm{c}}=c_{\mathrm{s}} .
$$

In summary, given that $v_{\mathrm{c}}$ is nonzero, and that we have a condensate (by construction) in this system, if $v<v_{\mathrm{c}}$, we can say that there is superfluidity. This result, however, is only valid for zero temperature.

Landau also developed the theory for a superfluid at finite temperature, the two-fluid model. At finite temperatures, the fluid has two components: the superfluid component 
that flows without friction and a normal fluid which describes the excitations. In this theory, then there are two sounds speeds, for each degree of freedom. In the case of weakly interacting Bose gas, the first sound is $c_{\mathrm{s}}$ associated with the oscillation in density, and the second sound is $c_{\mathrm{S}} / \sqrt{3}$ that corresponds to the speed of propagation of the temperature oscillations.

This phenomenological theory is still an important topic of research as a condition for superfluidity. From simulations to experiments, it is interesting to ask if the Landau criteria is fulfilled as a criteria for superfluidity. This criterion seems to be valid only in the regime of linear perturbations. This is the case since in Landau's theory the superfluid dissipates only into elementary excitations. However, we know that it is also possible to exist quantum vortices, topological defects present in rotating superfluids. This phenomenological theory does not take that into account, which can change the critical velocity of the superfluid to smaller values, reaching the dissipative regime of the superfluid before than if using Landau's critical velocity Ianeselli et al. (2006).

\section{Landau criteria for superflduidity}

Phenomenological conditions for superfluidity (at $T=0$ ):

1. Existence of a condensate;

2. $v_{\mathrm{c}} \neq 0 \quad$ (Non-interacting Bose gas has $v_{\mathrm{c}}=0$ - not a superfluid! Interaction is crucial for superfluidity.)

3. $v<v_{\mathrm{c}} \quad$-system transports charge without dissipation and the coherence of the BEC is maintained. $v_{\mathrm{c}}=$ velocity above which excitations can leave the condensate $\left(v_{\mathrm{c}}=c_{\mathrm{s}}\right.$ interacting BEC)

At finite temperatures, two fluid model: the superfluid component that flows without friction, and a normal fluid which describes the excitations.

\subsection{Weakly interacting Bose gas superfluid}

We now turn to the discussion of interacting systems. Inspired by Landau's phenomenological theory, the weakly interacting Bose gas was proposed as the simplest system to study superfluidity, and as a realistic model to understand condensation. In this section, we are going to model a superfluid by a Bose-Einstein condensate that has self-interaction, and show that, although a BEC can be formed both in the case of the non-interacting and interacting Bose gas, the presence of interaction is crucial for superfluidity (Pitaevskii and Stringari 2016; Barenghi and Parker 2016; Rogel-Salazar et al. 2004).

We present here a microscopic description of superfluid which arises upon condensation. The microscopic system where this happens is a weakly interacting gas of 
bosonic particles. To describe this interacting gas, first we need to understand how to describe the excitations in this system.

One of the conditions for condensation is that the gas is dilute. However, even in a dilute gas, the interaction can play an important role. The way we describe interactions or collisions in a Bose gas is somewhat different than in a classical fluid. Since now we describe it using their wavefunction, we need to have a interatomic potential $V_{\text {int }}$ to enable these collisions. In a dilute system at low temperatures, three-body interactions are suppressed, so we are going to describe this system with binary collisions. In such a system, the two-body collisions depend only on one parameter $a$, the $s$-wave (or coherent) scattering length (Harko and Lake 2015), which is the zero energy limit of the scattering amplitude $a=\lim _{T \rightarrow 0} f_{\text {scat }}$. This is valid only for low energies when the other length scales of the problem $d \gg a$. In this limit, the elastic scattering crosssection becomes constant $\sigma=8 \pi a^{2}$, and the two-body interatomic potential can be written as $V_{\text {int }}\left(\mathbf{r}-\mathbf{r}^{\prime}\right)=\left(4 \pi a \hbar^{2} / m\right) \delta\left(r-r^{\prime}\right) \equiv g \delta\left(r-r^{\prime}\right)$, which is short-ranged and present only when the atoms interact. The s-wave scattering length $a$ can be positive or negative depending on the system described, representing a repulsive or an attractive interaction. With this we can model the effective interaction Hamiltonian as $\hat{H}_{\text {int }}=$ $\int d \mathbf{r} d \mathbf{r}^{\prime} \hat{\Psi}^{\dagger}(\mathbf{r}) \hat{\Psi}^{\dagger}(\mathbf{r}) V_{\mathrm{int}}\left(\mathbf{r}-\mathbf{r}^{\prime}\right) \hat{\Psi}\left(\mathbf{r}^{\prime}\right) \hat{\Psi}\left(\mathbf{r}^{\prime}\right) \approx \int \mathrm{d}^{3} r \hat{\Psi}^{\dagger}(\mathbf{r}) \hat{\Psi}^{\dagger}(\mathbf{r}) \hat{\Psi}(\mathbf{r}) \hat{\Psi}(\mathbf{r})$, where $\Psi$ is the Bose operator.

The dynamics of this many-body interacting system is given by the secondquantized $N$-body Hamiltonian,

$$
\hat{H}=\int \mathrm{d}^{3} r \hat{\Psi}^{\dagger}(\mathbf{r})\left[-\frac{\hbar^{2} \nabla^{2}}{2 m}+V_{\text {trap }}(\mathbf{r})\right] \hat{\Psi}+\frac{g}{2} \int \mathrm{d}^{3} r \hat{\Psi}^{\dagger}(\mathbf{r}) \hat{\Psi}^{\dagger}(\mathbf{r}) \hat{\Psi}(\mathbf{r}) \hat{\Psi}(\mathbf{r}),
$$

where the brackets is the single particle Hamiltonian, and $V_{\text {trap }}(\mathbf{r})$ is the trapping potential, an external potential applied to the system (that in the next section could be the gravitational potential). In the Heisenberg description, we can then write the Heisenberg equations of motion,

$$
i \hbar \frac{\partial \hat{\Psi}(\mathbf{r}, t)}{\partial t}=[\hat{\Psi}(\mathbf{r}, t), \hat{H}]=\left(-\frac{\hbar^{2}}{2 m} \nabla^{2}+V_{\text {trap }}(\mathbf{r})\right) \hat{\Psi}(\mathbf{r}, t)+g \hat{\Psi}^{\dagger}(\mathbf{r}, t) \hat{\Psi}(\mathbf{r}, t) \hat{\Psi}(\mathbf{r}, t)
$$

with the brackets indicating the commutator. This is the Schrödinger equation for the Bose field operator $\hat{\Psi}(\mathbf{r}, t)$.

The Bose field operator $\hat{\Psi}^{\dagger}(\mathbf{r})$ and $\hat{\Psi}(\mathbf{r})$ create and annihilate a particle at position $\mathbf{r}$, and obeys the canonical commutation relations with only non-zero commutator given by $\left[\hat{\Psi}(\mathbf{r}), \hat{\Psi}^{\dagger}(\mathbf{r})\right]=\delta\left(\mathbf{r}-\mathbf{r}^{\prime}\right)$. The Bose field operator describes a continuum spectrum of single particle position eigenstates, and can be re-written in the singleparticle basis as

$$
\hat{\Psi}^{\dagger}=\sum_{i} \hat{a}_{i}^{\dagger} \phi_{i}^{*}(\mathbf{r}), \quad \hat{\Psi}=\sum_{i} \hat{a}_{i} \phi_{i}(\mathbf{r})
$$


where $\phi_{i}$ is the states wavefunction, and the creation and annihilation operators, $\hat{a}_{i}^{\dagger}$ and $\hat{a}_{i}$, create and annihilate a particle from the state $\phi_{i}$. They obey the Bose commutation relations, ${ }^{11}$ with only non-zero component given by $\left[\hat{a}_{i}, \hat{a}_{j}^{\dagger}\right]=\delta_{i j}$.

Many-particle systems described by the Hamiltonian (20) are very difficult to be solved. With the exception of a few simple models, to find solutions to this problem and be able to study its properties we need to make simplifications. For that we use Bogoliubov's prescription or mean-field approximation. For the general case of a nonuniform gas, the mean-field approximation can be written, in the Heisenberg picture as

$$
\hat{\Psi}(\mathbf{r}, t)=\psi(\mathbf{r}, t)+\delta \hat{\Psi}(\mathbf{r}, t),
$$

where $\psi(\mathbf{r}, t) \equiv\langle\hat{\Psi}(\mathbf{r}, t)\rangle$ is classical field called the wavefunction of the condensate. The density of the condensate is fixed by: $n_{0}=|\psi(\mathbf{r}, t)|^{2}=n$. Like we described for Landau's theory, $\delta \hat{\Psi}(\mathbf{r}, t)$ is a small perturbation of the system with $\langle\delta \hat{\Psi}(\mathbf{r}, t)\rangle=0$ and describes depletion of the condensate.

Effectively this approximation leads the many-body problem to be reduced to a single body problem by describing the averaging the effects of all other particles. Given that the interactions are weak, and that the gas is diluted, quantum fluctuations on the condensate are suppressed. The mean field approximation is valid for dilute system with $n a^{3} \ll 1$. When this conditions is not met there are deviations of the meanfield approximation. We can treat these deviations in perturbation theory, where we invoke non-vanishing moments for the fluctuation operator, like the Hartree-FockBogoliubov, which considers a non-zero $\langle\delta \hat{\Psi}\rangle$, or the Hartree-Fock-BogoliubovPopov, for terms up to second order in the perturbation.

With this approximation, we can write the generalized Gross-Pitaevskii (GP) equation:

$$
i \hbar \frac{\partial \psi(\mathbf{r}, t)}{\partial t}=\left(-\frac{\hbar^{2}}{2 m} \nabla^{2}+V_{\text {trap }}(\mathbf{r})+g|\psi(\mathbf{r}, \mathbf{t})|^{2}\right) \psi(\mathbf{r}, t) .
$$

The GP equation is a non-linear Schrödinger equation, with non-linearity arising from the self-interaction term. This equation describes the dynamics of the zero-temperature dilute weakly-interacting Bose system by allowing us to determine the shape of the single particle wave function, the condensate.

We can study the case of stationary solutions. The stationary solution can be taken as the solution that provides us with the condensate, the ground state wavefunction. The ground state is the lowest energy state of a quantum mechanical system, with the excited states being the states with higher energy than the ground state. The stationary

${ }^{11}$ In terms of the creation and annihilation operator, the Hamiltonian of the many-body system is given by,

$$
\hat{H}=\sum_{i j} H_{i j}^{\mathrm{sp}} \hat{a}_{i}^{\dagger} \hat{a}_{j}+\frac{1}{2} \sum_{i j k m}\langle i j|\hat{V}| k m\rangle \hat{a}_{i}^{\dagger} \hat{a}_{j}^{\dagger} \hat{a}_{k} \hat{a}_{m},
$$

where $\langle i j|\hat{V}| k m\rangle$ denotes the matrix element for the interaction, and $H_{i j}^{\mathrm{sp}}=\int \mathrm{d}^{3} \mathbf{r} \tilde{\Phi}_{i}^{*}(\mathbf{r}) \hat{H}^{\mathrm{sp}} \tilde{\Phi}_{j}(\mathbf{r})$, where $\tilde{\Phi}_{j}$ is the states wavefunctions. 
states $\Psi$ are the eigenfunctions of the Hamiltonian operator, with eigenvalues $\mu$ related to the energy of the system, in a way that for the wavefunction we have

$$
i \hbar \frac{\partial \Psi}{\partial t}=\hat{H} \Psi=\mu \Psi
$$

With that we can write the stationary solution as

$$
\psi(\mathbf{r}, t)=\psi_{\mathrm{s}}(\mathbf{r}) e^{-\frac{i}{\hbar} \mu t}
$$

where the eigenvalue of the Hamiltonian $\mu$ is also called the chemical potential, and $\phi_{\mathrm{s}}$ is real field with $\int d \mathbf{r} \psi^{2}=N_{0}=N$. The Gross-Pitaevskii equation becomes

$$
\left(-\frac{\hbar^{2}}{2 m} \nabla^{2}+V_{\text {trap }}(\mathbf{r})+g\left|\psi_{\mathrm{s}}(\mathbf{r})\right|^{2}\right) \psi_{\mathrm{s}}(\mathbf{r})=\mu \psi_{\mathrm{s}}(\mathbf{r}) .
$$

In the Thomas-Fermi limit, which is the approximation where the interaction energy is bigger than the kinetic energy for a large number of particles, the kinetic energy can be neglected so we have $\mu \psi=\left(g n+V_{\text {trap }}\right) \psi$. As a solution of this equation, we get that in the Thomas-Fermi limit,

$$
n(\mathbf{r})=|\psi(\mathbf{r})|^{2}= \begin{cases}\left(\mu-V_{\text {trap }}(\mathbf{r})\right) / g, & \text { for } \mathbf{r} \text { where }\left(\mu-V_{\text {trap }}(\mathbf{r})\right) / g>0, \\ 0, & \text { otherwise }\end{cases}
$$

\section{Fluid description}

We can decompose the complex macroscopic condensate wavefunction into

$$
\psi(\mathbf{r}, t)=|\psi(\mathbf{r}, t)| e^{i \theta(\mathbf{r}, t)},
$$

where, as we saw above, $\psi$ is normalized to the total number of particles, $|\psi(\mathbf{r}, t)|=$ $\sqrt{n(\mathbf{r}, t)}=\sqrt{\rho(\mathbf{r}, t) / m}$, and $\theta(\mathbf{r}, t)$ is the phase distribution. Inserting this into the GP equation, we get two equations. We make the following redefinition

$$
\mathbf{v}(\mathbf{r}, t) \equiv \frac{\hbar}{m} \nabla \theta(\mathbf{r}, t)
$$

This, together with (30) is called Madelung transformation. With those new variables, the GP equation results in two equations, the Madelung equations (Madelung 1927):

$$
\begin{aligned}
& \frac{\partial \rho}{\partial t}+\nabla \cdot(\rho \mathbf{v})=0 \\
& \rho \frac{\partial \mathbf{v}}{\partial t}+\rho(\mathbf{v} \cdot \nabla) \mathbf{v}=-\nabla\left(P_{\text {int }}+P_{\mathrm{QP}}\right)-n \nabla V_{\text {trap }} .
\end{aligned}
$$

They are a representation of the GP equation in "hydrodynamical" equations since they have a similar form as the continuity equation and Euler equation for a perfect 
fluid. However, the second Madelung equation describes a fluid with a potential flow, given the definition of the velocity, with zero vorticity $\nabla \times \mathbf{v}=\mathbf{0}$. This corresponds to the main characteristic of the superfluid that it flows without friction, has irrotational flow. This equation also differs from the perfect fluid Euler equation by the presence of the quantum pressure term.

The second Madelung equation, the Euler-like equation, reveals more interesting properties of the superfluid. This equation has two pressure terms, $P_{\text {int }}$ and $P_{\mathrm{QP}}$ that are respectively, the pressure term, and the quantum pressure:

$$
\begin{gathered}
P_{\mathrm{int}}=\left.K \rho^{(j+1) / j}\right|_{j=1}=\frac{g}{2} n^{2}=\frac{g}{2 m^{2}} \rho^{2}, \\
\nabla P_{\mathrm{QP}}=-n \nabla Q=-n \nabla\left[\frac{\hbar^{2}}{2 m^{2}} \frac{\nabla^{2} \sqrt{n}}{\sqrt{n}}\right] .
\end{gathered}
$$

The pressure term comes from the self-interaction which gives a polytropic type of pressure. For the two-body interaction, which is the case we show here, $j=1$. If we have a three-body interaction, for example, the pressure would have polytropic index $j=1 / 2$, giving $P_{\text {int }} \propto \rho^{3}$. The constant $K$ depends on the interaction constant. The quantum pressure is defined in terms of the quantum potential $Q=-\left(\hbar^{2} / 2 m\right) \nabla^{2} \sqrt{n} / \sqrt{n}$ (see definition below).

Quantum pressure: Quantum pressure $(\mathrm{QP})$ is the name given to the term ${ }^{12}$

$$
\nabla P_{\mathrm{QP}}=-n \nabla\left[\frac{\hbar^{2}}{2 m} \frac{\nabla^{2} \sqrt{n}}{\sqrt{n}}\right], \quad P_{i j, \mathrm{QP}}=-\left(\frac{\hbar}{2 m}\right)^{2} \rho \partial_{i} \partial_{j} \ln \rho,
$$

where $P_{i j}$, QP is the quantum pressure tensor. Together with the second term on the left hand side of equation (33) this term comes from the spatial part of the kinetic term. However, those two components are very different. The classical component describes the kinetic pressure due to the motion of the particle. The quantum pressure comes from the quantum part of the kinetic term that arises due to the Heisenberg uncertainty principle or can be seen as the curvature of the amplitude of the wave function. This is an additional force term that appear in the Madelung equation due to the zero point motion of particles. In the mean field approximation, this term is still present, given the classical wave function describing the system, so the term quantum is also misleading in this context. This term is repulsive and counter-acts attraction from a potential or attractive interaction, supporting the system against collapse. With that, the system cannot have vanishing size. This term modifies the dispersion relations of the excitations of the condensate and it is important at small scales, for scales smaller than the healing length. This term is negligible for large scales, the 
Thomas-Fermi approximation, and for a uniform superfluid, since $n=$ const..

Healing length: $\quad$ defined as the length for which $P_{\mathrm{int}}=P_{\mathrm{PQ}}$, given by,

$$
\xi=\frac{\hbar}{\sqrt{2 m g n}} .
$$

It is the length for which the interactions "heal" (coarse-grain) any density of phase perturbations in the condensate.

\section{Condensate solution}

Having established how we describe the weakly interacting system in the mean-field approximation, we can now describe what is the ground state, the condensate. We can solve analytically the GP equation for a few simple cases and obtain the condensate solution as for the cases with no interactions, in harmonic potential, and other simple systems (Barenghi and Parker 2016). We are going to present some interesting cases here.

In the case of a uniform gas, the wavefunction $\Phi_{0}(\mathbf{r})=\sqrt{N} \phi_{0} / \sqrt{V}$ describes the condensate formed, while the remaining functions form a complete set of functions orthogonal to the condensate.

Solitons Solitons are a localized solution of the one-dimensional GP equation with $V_{\text {trap }}=0$, which is integrable in this limit. They are also called solitary waves, and are solutions described by a permanent and localized wave, that maintains its shape and velocity upon collisions. They are obtained when the dispersion term, the kinetic spatial term, and the non-linear term from the interaction cancel out. If the interaction is repulsive $(g>0)$, a dark soliton is formed,which is given by the solution $\psi_{\mathrm{d}}(x)=\psi_{0} \tanh (x / \sqrt{2 \xi})$, where $\psi_{0}=\lim _{x \rightarrow \infty} \psi$. A bright soliton is the solution for attractive interactions $(g<0)$, with $\psi_{\mathrm{b}}(x)=\psi_{0} e^{-i \mu t / \hbar} \cosh (\sqrt{2 m|\mu| / \hbar} x)^{-1}$. They are called dark and bright since they represent a decrease and a concentration of condensate, respectively.

\section{Collective excitations, dispersion relation and sound speed}

The excitations in the superfluid which represent perturbations of the condensate, are an important part of this system. They represent sound waves, called the phonons that propagate through the condensate. We are going to show how they arise.

Here we are going to work in the case $V_{\text {trap }}=0$, for simplicity. The case where a trapping potential is present, like for example a condensate in the presence of a gravitational potential, is studied in Sect. 4.1. For a homogeneous condensate, to study the perturbations around the condensate, we perturb the classical wavefunction

\footnotetext{
12 One can notice that the form of the quantum potential coming from the Madelung equations, and consequently the quantum pressure tensor, are very similar to the Bohm quantum potential (Bohm 1952a, b). Some authors point that Bohm rediscovered the quantum pressure in his new interpretation of quantum mechanics. However, some authors claim this equivalence is not so clear. Some authors also claim that since the quantum pressure tensor has non-diagonal components, and it is not isotropic, so it cannot be called pressure (Hui et al. 2020).
} 
$\psi(\mathrm{r}, t)$,

$$
\psi(x, t)=\psi_{0}+\delta \psi^{(1)}+\delta \psi^{(2)}+\cdots,
$$

where we assumed that the motion is only in the $x$-directions without lost of generalization. The perturbations are small and $\delta \psi^{(i)}$ indicate the $i^{\text {th }}$ order in perturbation. To linear order, we can re-write the linearized GP equation: $i \hbar \partial_{t} \delta \psi^{(1)}=$ $-\left(\hbar^{2} / 2 m\right) \partial_{x}^{2} \delta \psi^{(1)}+\mu\left(\delta \psi^{(1)}+\delta \psi^{*(1)}\right)$. We make an ansatz for the solution as travelling waves, $\delta \psi^{(1)}=A e^{i\left(k x-\omega_{k} t\right)}+B e^{-i\left(k x-\omega_{k} t\right)}$. The parameters $A, B$ are determined by the initial conditions. Substituting this ansatz into the linearized GP equation, we can see that the dispersion relation is given by

$$
\omega_{k}^{2}=c_{s}^{2} k^{2}+\frac{\hbar^{2}}{(2 m)^{2}} k^{4},
$$

where the sound speed is defined as the term coming from the linear part of the dispersion relation

$$
c_{s}^{2}=\frac{g n_{0}}{m} .
$$

The sound speed appears because of the presence of the interaction; therefore, since for a superfluid the presence of an interaction is crucial, we can say that a superfluid is a fluid that has a sound speed. With this we can easily see the definition and properties of a superfluid.

Superfluid: In the presence of interactions, a sound speed is present which determines the behaviour of the excitations on large-scales. - For large wavelengths (small $k$ ), the higher $k$ terms do not contribute and the dispersion relation is given by

$$
\omega_{k}=c_{s} k
$$

which is the dispersion relation of a sound wave. The superfluid is characterized by excitations that propagate as waves, the phonons. Because of this property phonons can mediate a long- range forces $\left(F \sim 1 / r^{2}\right)$. This long-range force is the responsible for the effective dynamics of a superfluid: flowing without friction. We are going to see later (in the field theory description) that this gapless mode can also be viewed as the Nambu-Goldstone modes coming from the spontaneous symmetry breaking of U(1) symmetry of the system caused by the formation of the BEC.

This limit where the quantum pressure term can be ignored, or more specifically when $P_{\text {int }} \gg P_{\mathrm{QP}}$, is the Thomas-Fermi approximation we saw above, and can alternatively be defined by wavelengths bigger than the healing length.

This is the case for a repulsive interaction $(g>0)$. The situation is different for an attractive interaction $(g<0)$, where $w_{k}$ is imaginary and the solutions is unstable given by exponentially growing or decaying functions. This means that it is not 
possible to form a stable condensate in these cases.

For small wavelengths (large $k$ ), the quantum pressure term dominates, and the dispersion relation is given by $\omega_{k}=\hbar k^{2} / 2 m$, which describes a free particle. In this limit, the system stops exhibiting superfluidity.

In general, for intermediary frequencies, the full dispersion relation (38) does not propagate as a wave and shows two degrees of freedom: a gapless mode (the phonon), that propagates as a wave, and a massive mode, related to particle creation. We are going to discuss these again in the field theory section.

No interactions-BEC: In the limit where $g \rightarrow 0$, the BEC stops exhibiting superfluidity. The phonon becomes gapped, the dispersion relation is given by $\omega_{k}=\hbar k^{2} / 2 m$, which is the dispersion relation of a free particle. Since $\omega_{k}^{2}>0$ in this case, the solution of the linearized GP equation (without a external potential) is a stable oscillatory solution.

We showed above that the superfluid can decay into collective elementary excitation, the long-wavelength sound-wave quanta, the phonon. These are excitations with linear dispersion relation that behave as periodic fluctuations in density in the superfluid. When studying linear perturbations around a classical condensate background, only the phonon excitation is expected. However, a superfluid can also deplete into other excitations called rotons and maxons. The linear part of the dispersion relation is only a part of the dispersion relation at small momenta. For higher momenta, the dispersion relation presents a maximum and then a minimum. Near the maximum we have the maxons excitations, and near the minimum, for even higher momenta, we have the roton excitations. So, phonons, rotons and maxons are excitations described by different parts of the same dispersion relation. And lastly, for a rotating superfluid, there is also the vortex nucleation of the condensate, as we will see in Sect. 3.5.

$U(1)$ symmetry group: The unitary group of degree $n=1$ is the group associated with $n \times n$ unitary matrices $\left(U^{*} U=U U^{*}=1\right)$ under matrix multiplication, $U=e^{i \alpha}$, with $\alpha$ the parameter of the group. For $n=1$, this is the unitary transformations complex numbers, which corresponds to phase rotations. The $U(1)$ group is isomorphic ${ }^{13}$ to the $S O(2)$, the group of the $2 \times 2$ orthogonal rotations in $\mathbb{R}^{2}$ with unit determinant. The U(1) symmetry can be global, when it acts in the same way in all points in space-time, or local, acts differently at each place in space and time.

Noether's theorem: One of the most important results theoretical physics, Noether's theorem definition can be found in basically every textbook in field theory and even classical mechanics. The theorem states that for each continuous symmetry of a action there is an associated conserved current $j^{v}(\mathbf{x}, t)$, $\partial_{\nu} j^{v}(\mathbf{x}, t)=0$. This has profound implications since the conservation laws that describe the dynamics of a systems are then associated with the symmetries present in the problem. 
We can illustrate this for a system with the global U(1) symmetry that, according to Noether's theorem has a conserved particle number density ${ }^{14}$. Consider a complex scalar field $(\Psi(\mathbf{x}, t))$ theory with action:

$$
S=\int d^{4} x \mathcal{L}=\int d^{4} x\left[\left(\partial_{\mu} \Psi\right)^{*}\left(\partial_{\mu} \Psi\right)+V(\mathbf{x}) \Psi^{*} \Psi\right],
$$

where $V(\mathbf{x})$ is a potential. On top of being invariant under Lorentz transformations, this system has U(1) symmetry, with the action invariant under continuous rotations of the phase of the complex scalar field: $\Psi \rightarrow e^{i \alpha} \Psi$ (and c.c.). Since this is a global symmetry, $\alpha$ is independent of $(\mathbf{x}, t)$. With that, we can evaluate the Noether current and charge of this system:

$$
j^{v}=i\left(\Psi^{\dagger} \partial^{v} \Psi-\Psi \partial^{v} \Psi^{\dagger}\right), \quad Q=\int d^{3} x j^{0},
$$

which are conserved: $\partial_{\nu} j^{v}=0$ and $d Q / d t=0$. From $j^{v}=(m n, \mathbf{j})$, the conserved charge can be written in terms of the local density of $\mathrm{Q}, Q=$ $\int d^{3} x \rho(\mathbf{x}, t)$, and the conserved current implies a continuity equation for $\rho$. In this way, $Q \propto \Psi^{\dagger} \Psi$, which implies the conservation of the norm and it is associated with number conservation.

Some comments are in order. When defining $\psi$ as the condensate wavefunction, this quantity is actually a mean-field value of the wavefunction, the degree of freedom that defines the condensate. This description of averaging selecting the condensate is consistent with the theory of critical phenomena, like phase transitions. This Bose system can be seen as a system with spontaneous breaking of a symmetry of the description. In our case the U(1) symmetry which is the symmetry of the Hamiltonian. This is analogous to the the spontaneous symmetry breaking in a ferromagnet. The difference is that, since we have a Bose system, the idea of spontaneous symmetry breaking to the thermodynamical limit of a finite size Bose gas defines the number of particles of the system, and this is only consistent with the picture of having a condensate that can change the number of particles in the ground state, if the number of particles is conserved.

We can also understand this argument for the symmetry breaking of the system by analysing another approach to the weakly interacting system initially developed by Penrose and Onsager (1956), and Beliaev (1958). In this approach, the condensate wavefunction is identified, using the density matrix $\bar{\rho}\left(\mathbf{r}, \mathbf{r}^{\prime}\right)=\left\langle\hat{\Psi}\left(\mathbf{r}^{\prime}\right) \hat{\Psi}(\mathbf{r})\right\rangle$, to the number density of particles $n(\mathbf{r})=\bar{\rho}(\mathbf{r}, \mathbf{r})=\sum_{i} n_{i}|\phi(\mathbf{r})|^{2}$, where we are working in the stationary case. The formation of a BEC, which means that the ground state

\footnotetext{
13 Isomorphism is a one on one mapping between the elements of groups preserving its group operations. In the case of $U(1)$ and $S O(2)$, the isomorphism takes a complex number of unit norm into a rotation, $e^{i \theta} \mapsto\left(\begin{array}{cc}\cos \theta & -\sin \theta \\ \sin \theta & \cos \theta\end{array}\right)$, where the rotation angle is the argument of $z$.

14 For a more general derivation of Noether's theorem in field theory for any continuous symmetry and the connection with the generators of the symmetry group see Zee (2003).
} 
has a macroscopic occupation, leads to the factorization $N=n_{0}+\sum_{i \neq 0} n_{i}$, which means $\bar{\rho}\left(\mathbf{r}, \mathbf{r}^{\prime}\right)=\Phi_{0}\left(\mathbf{r}^{\prime}\right) \Phi_{0}(\mathbf{r})+\sum_{i \neq 0} n_{i} \phi_{i}^{*}\left(\mathbf{r}^{\prime}\right) \phi_{i}(\mathbf{r})$. The field operator then can be factorized in the presence of a condensate into $\hat{\Psi}(\mathbf{r})=\Phi_{0}(\mathbf{r})+\delta \hat{\Phi}(\mathbf{r})$, with $\langle\delta \hat{\Psi}(\mathbf{r})\rangle=0$. This means when a condensate form,

$$
\langle\hat{\Psi}(\mathbf{r})\rangle=\Phi_{0}(\mathbf{r}) \neq 0 .
$$

A state that has conserved particle number has $\langle\hat{\Psi}\rangle=0$. So this condition above is seen as describing a symmetry breaking, more specifically Bose symmetry breaking, and the consequence is a system where particle number is not conserved in the condensate. In the absence of a condensate, this goes back to the particle conserving condition:

$$
\Phi_{0} \begin{cases}=0, & \text { for } T>T_{\mathrm{c}} \\ \neq 0 & \text { for } T<T_{\mathrm{c}}\end{cases}
$$

This suggests that condensation comes from a spontaneous symmetry breaking theory.

With that, the interacting condensate system above can be understood as a particle conserving system of bosons with U(1) symmetry, described by the classical field $\psi$, the wavefunction of the condensate, where the formation of a Bose-Einstein condensate is a phase transition, coming from a spontaneous breaking of the symmetry (that can be seen as spontaneous coherence). This description of the condensate makes us see a parallel with the formalism used in field theory.

\subsubsection{Field theory description}

Given the suggestion that we can describe condensation as spontaneous symmetry breaking process, we turn now to the description of this system using a field theory language. The methods from field theory are very appropriate to describe this type of system where spontaneous symmetry breaking is present. Given the description we had above for the superfluid and the properties of this system, we represent this system as a massive complex scalar field ${ }^{15}$ with self-interactions with a global $U(1)$ symmetry that is spontaneously broken by the presence of the ground state, the condensate, with superfluidity arising upon condensation. We are going to work here, to best illustrate this description, in the homogeneous case, where there is no trapping or external potential applied to the system.

Given that, we describe this system by the Lagrangian density for a two-body interaction,

$$
\mathcal{L}=\left(\partial_{\mu} \Psi\right)^{*}\left(\partial_{\mu} \Psi\right)-m^{2} \Psi^{*} \Psi-\frac{g}{2}\left(\Psi^{*} \Psi\right)^{2}
$$

where we consider $g>0$ to get a stable condensate with long-range coherence, as discussed above. As we saw above, this system has a $U(1)$ global symmetry, which

\footnotetext{
$\overline{15}$ We have used here a complex scalar field that has a continuous symmetry since, as we saw before according to Noether's theorem, this system has a conserved charge. The $U(1)$ symmetry gives us a system with conserved particle number. For this reason it is essential to use a complex scalar field.
} 
means that it is invariant under continuous rotations of the phase of the complex scalar field,

$$
\Psi \rightarrow \Psi e^{i \alpha}, \quad \Psi^{*} \rightarrow \Psi e^{-i \alpha}
$$

where $\alpha$ is a constant ${ }^{16}$. The equation of motion is given by,

$$
\partial^{\mu} \partial_{\mu} \Psi+m^{2} \Psi+g|\Psi|^{2} \Psi=0 .
$$

To describe the condensate, like we did previously, we separate the condensate contribution to the perturbations, and assume the mean-field approximation,

$$
\Psi(\mathbf{x}, t)=\psi(\mathbf{x}, t)+\delta \Psi(\mathbf{x}, t)
$$

where $\psi(\mathbf{x}, t)$ is the background that gives the condensate field, and $\delta \Psi(\mathbf{x}, t)$ are the excitations of the condensate that are considered small with respect to the background solution.

As we saw in (27), the stationary solution determines the ground state of the system. Using that, the condensate wavefunction can be written as

$$
\psi=v e^{i \mu t}
$$

where $\theta_{\mathrm{bg}}=\mu t$ is the phase of the ground state where $\mu$ is the chemical potential. ${ }^{17}$ This is the background solution for the equation of motion (47) as long as,

$$
\mu^{2}=m^{2}+g v^{2}
$$

When we have a spontaneous symmetry breaking, only the ground state, the state that broke the symmetry, is not invariant under $U(1)$ anymore. This means that we still have a conserved current given by $j^{v}=i\left(\Psi^{\dagger} \partial^{v} \Psi-\Psi \partial^{v} \Psi^{\dagger}\right)$, as we saw above in the definition of SSB. Using the equations of motion one can see that this current is conserved. For the ground state (49), this current gives the number density: $n=2 \mu v^{2}$. In the field theory description, the conservation of the norm $\left(\int|\Psi|^{2}\right)$, related to the number density in the condensate and conservation of the number of particles in the condensate seen in the quantum mechanical approach above, is interpreted as a consequence of the global symmetry of the system which leads to a conserved Noether charge, $Q=\int j^{0} \propto \int n \propto \int|\Psi|^{2}$ (conservation of the number density of field quanta).

\footnotetext{
16 It is equivalent to re-write this, in a language of $S O(2)$ symmetry, where the complex field can be written as $\Psi=(1 / \sqrt{2})\left(\Psi_{1}+i \Psi_{2}\right)$, where the system as an invariance under rotations: $\left\{\begin{array}{l}\Psi_{1} \rightarrow \cos \alpha \Psi_{1}-\sin \alpha \Psi_{2} \\ \Psi_{2} \rightarrow \sin \alpha \Psi_{1}+\cos \alpha \Psi_{2}\end{array}\right.$

17 We identified the chemical potential in our superfluid as the time derivative of the ground state phase, $\mu=\partial_{t} \theta_{\mathrm{bg}}$. This is only valid if $\partial_{i} \theta_{\mathrm{bg}}=0$, which is the chemical potential in the frame where the superfluid moves with velocity $\mathbf{v}$. If this is not true, the chemical potential is given by $\bar{\mu}=\sqrt{\partial_{\nu} \theta_{\mathrm{bg}} \partial^{\nu} \theta_{\mathrm{bg}}}$, which is the chemical potential in the rest frame of the fluid.
} 
Spontaneous symmetry breaking (SSB): $\quad$ SSB is the phenomenon where the state of the theory is not invariant (not symmetric) under the symmetry transformations $(U)$ of the Hamiltonian (or action) that describes the system. This stable state $|\psi\rangle$ spontaneously broke the symmetry of the system. This mechanism offers an explanation for why there exist stable states, like a condensate, that do not respect the symmetries of a system. This allow for the existence of different symmetry related state $U|\psi\rangle$, of same energy but different phases defining a set of symmetry-broken states. To distinguish them, or their phases, we have the order parameter of the system, defined as $\mathcal{O}=[Q, \Phi]$, where a state breaks a symmetry $U=e^{i} \alpha Q$ if $\Phi$ exists. The order parameter can be used to identify if a symmetry was broken $\langle\mathcal{O}\rangle \neq 0$, where the system is said to have long range order, given by its two-point function $(C(\mathbf{x})$ being proportional to a constant. For unbroken systems, $\langle\mathcal{O}\rangle=0$, where the $C\left(\mathbf{x}, \mathbf{x}^{\prime}\right) \propto \exp \left(-\left|\mathbf{x}-\mathbf{x}^{\prime}\right| / l\right)$. The coherent length, $l$, is infinite when there is long range order ${ }^{18}$.

This is the background solution corresponding to the condensate. This ground state is responsible for spontaneously breaking the $U(1)$ global symmetry. And we can see that explicitly. Since this solution represents the ground state, we can compute the energy functional for this system,

$$
E=\int \mathrm{d}^{3} x \mathcal{U}=\int \mathrm{d}^{3} x\left(\partial_{0} \Psi \partial^{0} \Psi+\partial_{i} \Psi \partial^{i} \Psi+V_{e f f}\right)
$$

where $V_{e f f}=m^{2} \Psi^{*} \Psi+g \Psi^{*} \Psi$. The ground state is given by the stationary, minimum energy state. This can be found by finding the minimum of the energy (51), which amounts to finding the minimum of the potential energy. The set of solutions for the minimum of this potential energy is

$$
\left\{\begin{array}{lll}
v_{0, \mathrm{~s}}=0, & \text { for } m^{2}>0, & \text { Symmetry restaured } \\
v_{0, \mathrm{ssb}}= \pm \sqrt{\frac{\left|m^{2}\right|}{g}}, & \text { for } m^{2}<0, & \text { Symmetry broken - condensate }
\end{array}\right.
$$

The value of the minima $v_{0, \mathrm{ssb}}= \pm m / \sqrt{g}$ are called vacuum expectation value and are the value that the field assumes at the ground state, apart from a phase. As we can see

18 For a very complete and extensive review on SSB, see Beekman et al. (2019).

An alternative way of writing this Lagrangian that leave explicitly the spontaneous breaking of the symmetry by the finite charge:

$$
\mathcal{L}=\left|\left(\partial_{\mu}-i \mu\right) \Psi\right|^{2}-m^{2}|\Psi|^{2}-g|\Psi|^{4}
$$

This is equivalent to the usual way, we introduce the chemical potential in the Hamiltonian: $\mathcal{H}-\mu \mathcal{N}$, where $\mathcal{N}=j^{0}$ is the conserved charge. For this modified Lagrangian, the condensate would have trivial phase $\theta_{\mathrm{bg}}=0$. This is equivalent to what we did in the text where the Lagrangian had the canonical kinetic term, but the phase of the condensate had a time dependency with the chemical potential $\theta_{\mathrm{bg}}=\mu t$. 

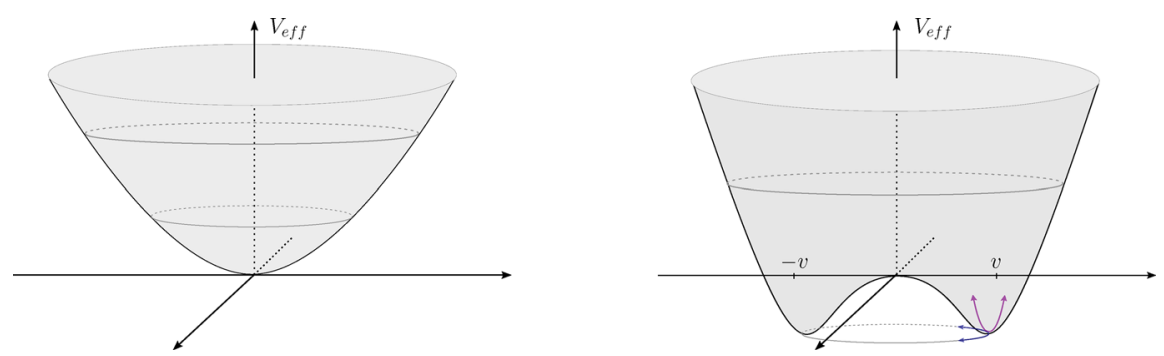

Fig. 7 Potential of the weakly self-interacting system. Left panel: Potential of the symmetry restored phase, $m^{2}>0$ which has a minimum at $\psi_{\mathrm{s}}=0$. This ground state is symmetric, respects the symmetry of the system. Right panel: Symmetry breaking potential, for $m^{2}<0$. This phase has a degenerate minima not invariant under the symmetry of the system. It represents the condensate state

from on the left panel of Fig. 7, the symmetry restored phase when $m^{2}>0$ has a welldefined minimum at $v_{0, \mathrm{~s}}=0$, and this is the normal phase, with no condensation. This ground state $\psi_{\mathrm{s}}=0$ is preserved under the symmetry of the system ( $U(1)$ symmetry), the rotations of the phase of the complex field.

The symmetry breaking phase when $m^{2}<0$, there is a continuous set of minima with the ground state given by $\psi_{\mathrm{ssb}}=v_{0, \mathrm{ssb}} e^{i \alpha}$, corresponding to all the possible phases in the circle $\alpha \in[0,2 \pi)$, as seen in the right panel of Fig. 7. All of these classical backgrounds are not invariant under $U(1)$ symmetry, the symmetry of the system, which means that $\phi_{i, \mathrm{ssb}} \rightarrow \phi_{j, \mathrm{ssb}}=v_{0, \mathrm{ssb}} e^{i \alpha} \neq \phi_{i, \mathrm{ssb}}$. In this way, we say that the symmetry is spontaneously broken by this condensate ground state. From the $\mathrm{SSB}$, we can see that the condensate has long-range order, with the field having the role of the order parameter of the system.

\section{Excitations}

We consider now fluctuations around the classical condensate configuration to study the spectrum of this system. Considering small fluctuations means perturbing each degree of freedom of the field around the condensate (48). This is equivalent in the polar notation to:

$$
\psi(\mathbf{x}, t)=(v+\rho) e^{i(\mu t+\pi)},
$$

where $\rho(\mathbf{x}, t)$ can be interpreted as a perturbation in the radial direction, and $\pi(\mathbf{x}, t)$ a perturbation in the angular/phase direction. Plugging this into the Lagrangian, we have

$$
\mathcal{L}=-\left(\partial_{\mu} \rho\right)^{2}+(v+\rho)^{2}\left[g v^{2}+2 \mu \dot{\pi}+\dot{\pi}^{2}-\left(\partial_{i} \pi\right)^{2}\right]-\frac{g}{2}(v+\rho)^{4} .
$$

With this expansion is already easy to see that $\rho$ has a mass term (the term accompanying the $\rho^{2}$ term), so the perturbation in the radial direction is massive. The perturbation in the phase has no mass term and it is going to be massless. This massless excitation was already expected from the Goldstone theorem (see below), where a SSB leads to the appearance of a massless excitation, the Nambu-Goldstone (NG) boson $\pi$. In the context of a superfluid, this gapless excitation is the phonon. 
For low-energy theories, the phonon is the only degree of freedom that is excited in the theory, as the massive mode can be integrated out. Therefore, at low energies a superfluid is completely described by the phonon excitations.

Low energies here mean energies lower than the mass gap of the massive mode $\rho$. We are going to work on this limit here to obtain the spectrum of this NG boson. In this limit, the kinetic term of $\rho$ can be neglected and the equation of motion becomes:

$$
g(v+\rho)^{2}=g v^{2}+2 \mu \dot{\pi}+\dot{\pi}^{2}-\left(\partial_{j} \pi\right)^{2} \equiv X .
$$

Using this, we can rewrite the on-shell Lagrangian in terms of $X$ as

$$
\mathcal{L}=\frac{X^{2}}{2 g}=\frac{1}{2 g}\left[g v^{2}+2 \mu \dot{\pi}+\dot{\pi}^{2}-\left(\partial_{j} \pi\right)^{2}\right]^{2} .
$$

This Lagrangian depends only on the phonon $\pi$, as $\rho$ was integrated out, and is invariant under shift symmetry, $\pi \rightarrow \pi+c$, inherited from the $U(1)$ symmetry of the complex scalar field. This is the effective Lagrangian at leading order in derivative expansion for the phonon. To obtain the dispersion relation we can expand this Lagrangian, using $\pi_{\mathrm{c}}=\mu \pi$,

$$
\begin{aligned}
\mathcal{L}= & \frac{1}{2 g} \frac{1}{4 \mu^{2}}\left\{g^{2} n^{4}+4 g n \mu \dot{\pi}_{\mathrm{c}}+\left(\frac{n g}{\mu}+4 \mu^{2}\right) \dot{\pi}_{\mathrm{c}}^{2}-\frac{n g}{\mu}\left(\partial_{j} \pi_{\mathrm{c}}\right)^{2}+2\left[\dot{\pi}_{\mathrm{c}}^{3}-\dot{\pi}_{\mathrm{c}}\left(\partial_{j} \pi_{\mathrm{c}}\right)^{2}\right]\right. \\
& \left.+\frac{1}{4 \mu^{2}}\left[\dot{\pi}_{\mathrm{c}}^{2}-\left(\partial_{j} \pi_{\mathrm{c}}\right)^{2}\right]^{2}\right\},
\end{aligned}
$$

where we used that $n=2 \mu v^{2}$. Taking the Fourier transform of the field, we compute the equation of motion and can see that the dispersion relation of the phonon is given by

$$
\omega_{k}^{2}=\frac{g n}{\mu} k^{2}+\frac{1}{(2 \mu)^{2}} k^{4}=c_{\mathrm{s}}^{2} k^{2}+\frac{1}{(2 \mu)^{2}} k^{4},
$$

where $c_{\mathrm{s}}$ is the sound speed of the superfluid. This expression shows us the general behaviour we already seen in the excitations calculated in the QM approach. For long wavelengths (small $\mathrm{k}$ ), the higher order term in $k$ is suppressed, and the dispersion relation is $w_{k \text {, long }}^{2}=c_{\mathrm{s}} k^{2}$, which is the dispersion relation characteristic of a sound wave. Therefore, the phonon propagates as a wave with sound speed $c_{\mathrm{s}}$ in the longwavelength regime.

The second term of the dispersion relation is characteristic of a massive particle. This means that for short wavelengths, the system behaves as a system of normal particles propagating, and not a superfluid.

We can re-write these expressions in the non-relativistic $(N R)$ regime, which is the regime we are interested in comparing to the previous approach (and also the limit where we describe, in the next section, the behaviour of DM in galaxies). In this limit, 
$g v^{2} \ll m^{2}$, which implies $\mu^{2} \approx m^{2}$. The dispersion relation in this case is given by

$$
w_{k, \mathrm{NR}}^{2}=c_{\mathrm{s}}^{2} k^{2}+\frac{1}{4 m^{2}} k^{4}, \quad \text { with } c_{\mathrm{s}}^{2}=\frac{g n}{m},
$$

which is equivalent to the one found in (38).

In the absence of interactions, we recover the ideal Bose gas from Sect. 3.1, with background solution is given by $\Psi_{\text {ideal }}=v e^{i m t}$, and dispersion relation of a massive particle $w_{k}^{2}=\left(1 / 4 m^{2}\right) k^{4}$, showing again that although this system condenses in to a $\mathrm{BEC}$, in the absence of interactions there is no superfluidity. It is easy to see from (58) that in the relativistic regime, $g v^{2} \gg m^{2}, c_{\mathrm{s}}^{2}=1 / 3$.

In this way, we showed that the field theory description of the superfluid is very good to describe the general properties of the superfluid. To properly compare with the results obtained above in the QM approach, we are going to show that in the nonrelativistic limit, the field theory description gives us the GP and Madelung equations obtained above.

Before doing that, one comment is in order. In (57), we showed that in the lowenergy regime, we can re-write the microscopic theory of a superfluid as an effective theory only of the phonon with a non-canonical kinetic term. We did this here in the case of a weakly self interacting system with two body interaction, but in Sect. 3.4 we are going to extend this idea to general superfluid systems and show the construction of the EFT of superfluids.

Goldstone theorem: The Goldstone theorem (Goldstone 1961; Goldstone et al. 1962; Nambu and Jona-Lasinio 1961) or Nambu-Goldstone theorem, states when a spontaneous symmetry breaking occurs a mode with energy that vanishes as $k \rightarrow 0$ is present in the spectrum of excitations of the system. This mode is called Nambu-Goldstone (NG) boson and is a massless particle, in the case of relativistic systems or collective excitations with zero energy gap for non-relativistic systems ${ }^{19}$.

When a symmetry is spontaneously broken, the Noether theorem still applies so there is still conserved currents. The stable state responsible for the SSB is not invariant under this conserved charge, $Q|\psi\rangle \neq 0$ (or $\langle\mathcal{O}\rangle \neq 0$ ). This condition implies that there must be a state, the NG mode, with $E_{\mathbf{k}} \rightarrow 0$ as $\mathbf{k} \rightarrow 0$, whose quanta is a massless boson. The NG boson still exists if the symmetry is not exact or broken by an external potential $\mu$, but in this case the mode has a gap $\mu$ at $k \rightarrow 0$. For ordinary NG bosons (type A), the number of NG bosons created is equal to the number of broken symmetry generators $Q$, $n_{\mathrm{BG}}$.

The Goldstone theorem described here is valid for a system that is invariant under Lorentz transformation, with the appearance of $n_{\mathrm{BG}} \mathrm{NB}$ bosons. However, SSB is an important phenomena in many systems that are not Lorentz invariant, like the BEC or supefluid, with a number of bosons that will appear in the theory, $n_{\mathrm{NG}}$, called Nambu-Goldstone (NG) bosons, which might not 
be equal to the number of broken generators, like in the Lorentz invariant case. A generalization of the Goldstone theorem, which includes systems that do not have Lorentz symmetry, exists and can be found in Watanabe and Brauner (2011); Watanabe and Murayama (2012). In these works they classify and generalize the Nambu-Goldstone theorem for any symmetry, including non-relativistic systems invariant under Galilean symmetry, showing how to compute the number of NG bosons created by the breaking of such symmetry.

\section{Recovering the other approaches}

We want to show that we can recover the GP theory presented in the previous subsection, and emphasize that the field theory description is compatible to describe the superfluid. The field theory presented above is a fully relativistic theory, which means that the action is invariant under Lorentz transformations on top of the global U(1). However, GP description shown above is non-relativistic. Therefore, to recover the GP and Madelung equations we need to take the non-relativistic limit of the relativistic field theory above. ${ }^{20}$

Starting from our field theory for the weakly interacting bosons (45), we take the non-relativistic limit of the Lagrangian. We do that by talking the limit $c \rightarrow \infty$ and assume that in this limit the field has a very fast phase rotation in time, which allows us to rewrite the fields as

$$
\Psi(\mathbf{x}, t)=\frac{1}{2 m} \psi(\mathbf{x}, t) e^{-i m t}
$$

With that, the Lagrangian can be written as

$$
\mathcal{L}=\frac{i}{2}\left(\dot{\psi} \psi^{*}-\psi \dot{\psi^{*}}\right)-\frac{1}{2 m}|\nabla \psi|^{2}-\frac{g}{16 m^{2}}\left(\psi^{*} \psi\right)^{2}
$$

From this non-relativistic Lagrangian, we can evaluate the equation of motion for the scalar field $\psi$,

$$
i \dot{\psi}=\left(-\frac{1}{2 m} \nabla^{2}+\frac{g}{8 m^{2}}|\psi|^{2}\right) \psi,
$$

which is exactly the Gross-Piatevskii equation like shown above, in the absence of a trapping/external potential.

\footnotetext{
19 Some translational symmetry needs to be maintained in the system, like Lorentz symmetry for relativistic systems, so that momentum is still a well-defined quantum number.

20 One can also already start from a non-relativistic action for a field, called Schrödinger field, which directly yields the Schrödinger equation. See Beekman et al. (2019) for this derivation.
} 
From that, we can also derive the Madelung hydrodynamical equations, if we make the following substitution:

$$
\psi \equiv \sqrt{\rho / m} e^{i \theta}, \quad \mathbf{v} \equiv \frac{1}{m} \nabla \theta
$$

The vorticity of the superfluid is zero and the momentum density has a non-zero curl. Plugging this in the equations of motion, we recover the Madelung equations (32) and (33) in the absence of an external potential. This shows again that we can recover the equations that describe the interacting BEC using the field theory approach.

In this section, we showed how to describe a weakly interacting BEC. We showed the standard treatment of the theory, where the many-body quantum system is described by the GP equation. We showed that condensation can be thought as a spontaneous symmetry breaking process and showed that the system can be described in a equivalent way using the field theory approach. We specialized in both cases in the mean-field theory, which is valid for dilute systems, and simplifies the significantly the study of the system.

It is important to comment on the validity of this theory and the approximations made. The theory presented above is only valid for zero temperatures and in the meanfield approximation that holds for $n a^{3} \ll 1$, where we can describe the condensate as a classical wavefunction and the limit where quantum corrections are not important. As we cited above, there are correction to the mean field and other approximations where one can study this model (see Pitaevskii and Stringari 2016 for some examples). For finite temperature, one has to describe the superfluid as a two-fluid model.

In the cases we are going to study, we will extend a bit the validity of the zero temperature description, as a first approximation, since in galaxies the temperature is obviously not zero. However, since the occupation number will be very high in our problem, the classical description is safe.

\section{Two-fluid model}

The description presented above for the superfluid is valid for a zero temperature dilute weakly interacting Bose gas. However, as already described in Landau's phenomenological theory, for finite temperature system, the superfluid has to be described as a two-fluid model: a mixture of a superfluid and a normal fluid. As we saw previously in this description, for $T<T_{c}$, the fluid is a mixture of normal and superfluid with most of the fluid in the superfluid phase, while for temperatures higher than $T_{c}$, the coherence of the superfluid is broken and the fluid is mostly in the normal phase.

Connecting Landau's theory and the microscopic description of a superfluid by a weakly interacting theory, a two-fluid relativistic theory of superfluid was developed. This can be linked to the non-relativistic one described above, and a "hydrodynamical" and field theory approaches are developed. We are not going to describe this in detail in this review since this is not used to describe the current ULDM models in the literature. However, the two-fluid description should be used for finite temperature systems to describe a superfluid and we believe it is important to describe realistic DM superfluid models. For a review of the two-field model, see Schmitt (2015). In Sect. 4, we present 
one work where the two-fluid formalism is used to describe the self-interacting BEC DM.

The weakly interacting Bose system studied in this section is the prototypical description of a BEC system that presents superfluidity. It is a microscopical description that shows the behaviour of the condensate and its excitations. But this description is limited and cannot be used to describe all the models of possible superfluids and realistic experimental systems. The interesting point is that this theory can be recast as a spontaneous symmetry breaking theory of U(1) symmetry of the many-body system. BEC and superfluidity are a consequence solely of the spontaneous symmetry breaking, independent on the specific model chosen. Therefore, this hints us to describing these symmetry breaking systems using the effective field theory approach, where the Lagrangian of the system is described by symmetry alone. The hope is to be able to describe more complicated superfluid systems. We are going to describe this approach then in the next subsection.

\subsection{Effective field theory of a superfluid}

We described in the previous section the construction of a microscopic effective theory for the weakly interacting BEC that can be used to describe superfluids. This description is based on London's idea (London 1938), which has its roots in the superfluid/superconductor hydrodynamics, that the BEC can be described by a theory with spontaneous symmetry breaking caused by the condensate, with superfluidity arising upon condensation and being described by the Goldstone boson, the phonon, at low energies. We saw that we can write an effective Lagrangian for the phonon that describes the behaviour of the superfluid, matching many observations, in the low-energy and low-momentum regimes.

This procedure shows us that we can describe the hydrodynamical degrees of freedom of a theory by the Goldstone modes created by the SSB of a symmetry, the global $U$ (1) symmetry in our case. This is more general than the simple weakly interacting two-body interaction case showed above. This is already the case of hydrodynamics that describes macroscopically the behaviour of low-energy variables and interactions of system given a symmetry, coarse-graining over the smallest scales. This is the perfect playground for the use of effective field theories (EFT), and EFT techniques are very appropriate for this task. An EFT describes, the low energy (long distances) behaviour of a system, without having to refer to its underlying microscopic theory, by parametrizing our ignorance of those short scales.

The idea is to use EFT methods to describe the dynamics of a superfluid. This was developed in Son (2002); Dubovsky et al. (2006, 2012); Son and Wingate (2006), where they develop the general formalism to describe fluid hydrodynamics without dissipation as an EFT. In this approach the Lagrangian that describes the system is constrained by symmetry alone. ${ }^{21}$ This approach is very powerful since it not only can be applied to many different systems, but describes the system without the need

21 There are many other references that also develop EFTs for different condensed matter systems and under different conditions, as it can be seen in these references (Zakharov et al. 1992; Burgess 2000; Hofmann 1999), including discussions on dissipation, generalizations and modelling the UV physics that affects the EFT. This is not an extensive list, but showing just some examples of these constructions. For an EFT of 
of its microscopic understanding. It is also very powerful since it is an expansion over momentum. At leading order in the expansion, we describe the low-energy theory. But this description allows us to go beyond leading order in the long-wavelength expansion.

In our case, we want to construct the EFT that reproduces, in the long wavelength regime (low energies), the superfluid hydrodynamics, as presented above. This theory is a theory of the phonon, which is the only degree of freedom that is excited at low energies. This is the Goldstone mode produced by the SSB of the $U(1)$ global symmetry by the ground state. We are going to work here in the non-relativistic regime, but one can see the references above for the relativistic case. Restricting to the nonrelativistic regime does not imply any loss of generality of the argument, with the system only subjected to different symmetries than the relativistic case.

Inheriting the knowledge of a superfluid from previous sections, at low energies, the only dynamical degree of freedom that describes a superfluid is the phase of the condensate, the phonon. Therefore, in the non-relativistic regime, we need to construct the EFT of this phase $\pi$. The superfluid is described by a theory where the symmetry is spontaneously broken by the ground state,

$$
\theta=\mu t-\pi
$$

The theory that is described by this phase $\mathcal{L}_{\text {eff }}(\theta)$ is invariant under shift-symmetry $\theta \rightarrow \theta+c$ which is inherited from the $U(1)$ symmetry of the complex scalar field.

EFT states that to construct the effective Lagrangian for the phonon, we have to write all the terms that are compatible with the symmetries of the problem. This system has shift symmetry and Galilean symmetry. For the shift symmetry, for the Lagrangian to be invariant under this symmetry, only terms that are acted by a derivative can appear in the theory: $\mathcal{L}=\mathcal{L}\left(\dot{\theta}, \partial_{i} \theta\right)$. This may contain terms with any power of the derivative of the field.

However, this theory has more symmetries. In a generic space-time and adding a gauge field, which is a natural extension of the simple scalar field model, we require that this Lagrangian is invariant with respect to three-dimensional general coordinate transformations and gauge invariance. The most general Lagrangian $\mathcal{L}=\mathcal{L}\left(D_{t} \theta, g^{i j} D_{i} D_{j} \theta\right)$ that is invariant under these symmetries, shift symmetry, gauge invariance and general coordinate invariance, is given by,

$$
\mathcal{L}=P(X), \quad \text { with } \quad X=D_{t} \theta-\frac{g_{i j}}{2 m} D_{i} \theta D_{j} \theta
$$

where $D_{t} \theta=\dot{\theta}+A_{0}$ and $D_{i} \theta=\partial \theta-A_{i}$. In flat space, $g_{i j}=\delta_{i j}$, the general coordinate invariance corresponds to a Galilean symmetry.

As it can be seen in Son and Wingate (2006), Galilean symmetry is not enough to constrain the NLO terms and one needs to consider the full general coordinate invariance. This is equivalent to considering an additional constraint for the theory,

pions, one can check the following references (Gell-Mann 1962; Weinberg 1966; Gell-Mann et al. 1968; Weinberg 1979, 2009), as some examples. 
which is known from fluid hydrodynamics,

$$
T_{0 i}=m j_{i}
$$

where $T_{0 i}$ is the off-diagonal component of the energy-momentum tensor. As stated in Greiter et al. (1989) and Dubovsky et al. (2012), this additional constraint (with an analogous constraint in the case of relativistic systems, Dubovsky et al. 2012) states that only one degree of freedom carries all the current and momentum. Introducing a new symmetry, full general coordinate invariance is equivalent to assuming this relation.

With the two symmetries of the system, in the absence of gauge fields $A_{0}=A_{i}=0$, the Lagrangian that describes this system is given by

$$
\mathcal{L}=P\left(\dot{\theta}-\frac{\left(\partial_{i} \theta\right)^{2}}{2 m}\right)
$$

This is a Lagrangian that has a non-canonical kinetic term and it obeys (67).

At the background $\theta=\mu t$, and $T=0$, this Lagrangian density is equal to the pressure $P=P(\mu)$. With that we can evaluate the particle number density,

$$
n=P^{\prime}(X)
$$

where' indicates the derivative with respect to $A_{0}$. For the condensate, then the equilibrium number density at chemical potential $\mu$ is $n(\mu)=P^{\prime}(\mu)$, where $P(\mu)$ is the thermodynamical pressure, defined up to a constant.

Given the Lagrangian (68) with $\theta=\mu t+\phi$, we can write the Lagrangian as:

$$
L=P(\mu)-n \dot{\phi}+\frac{1}{2} \frac{\partial n}{\partial \mu} \dot{\pi}^{2}-\frac{n}{2 m}\left(\partial_{i} \pi\right)^{2} .
$$

We can see from that the phonon speed of sound

$$
c_{\mathrm{s}}=\sqrt{\frac{n}{m} \frac{\partial \mu}{\partial n}}=\sqrt{\frac{\partial P}{\partial \rho}},
$$

where $\rho=m n$ is the mass density.

One limit of this EFT Lagrangian is the quadratic Lagrangian for the weakly interacting BEC, shown in (57). We can see that by considering the special case where $P(X)$ is written as a polynomial, $P(X) \propto(\dot{\theta} / m)^{n}$. Depending on the power chosen we will have a superfluid with a different equation of state:

$$
\left\{\begin{array}{lll}
n=2: & P \sim \rho^{2} & \text { BEC/Sf }(2-\text { body }) \\
n=3 / 2: & P \sim \rho^{3} & \\
n=5 / 2:, & P \sim \rho^{5 / 3} & \text { Unitary Fermi gas }
\end{array}\right.
$$


These represent different systems. The case $n=2$ is equivalent to the previous case, in Sect. 3.3, where we had a superfluid with a two-body interaction described by the microscopic weakly interacting theory, where we obtained $P(X)=X^{2}$ for the low-energy (57). The case with $n=3 / 2$ can correspond to the same theory as the previous case, a weakly interacting theory, but with a three-body interaction, with this effective Lagrangian obtained by integrating out the massive radial mode, like done in the previous section. These two equivalences shows us an interesting aspect of this EFT and from the hydrodynamics of superfluids: the interaction is linked to the equation of state of the superfluid, and this can be seen by a different choice of $P(X)$ in the EFT. The case $n=3 / 2$ can also represent another completely different superfluid system, like we will see in the case of the superfluid DM in the next section, where the this case does not come from a weakly interacting microscopic theory with three-body interaction. This is also the case for the other example we show here, the unitary Fermi gas, which cannot be described by a microscopic theory like we did in the previous section, and being described with this EFT if $n=5 / 2$.

One comment is in order here. Usually in quantum field theory having fractional exponents can be problematic, leading to caustics or superluminal propagation. However, in the case of the superfluid, this is not a problem. Before reaching these regimes (like the formation of caustics), the superfluid coherence is broken, and the EFT description of the superfluid is no longer valid.

If one wants to add an external or trapping potential $V_{\text {ext }}$, like, for example, if the gas is under the influence of a gravitational potential, this corresponds to making $A_{0}=V_{\text {ext }}$. With that, the Lagrangian is given by (66), with $X=\dot{\theta}-\frac{\left(\partial_{i} \theta\right)^{2}}{2 m}-V_{\text {ext }}$. In the case of the condensate in a gravitational potential, this is given by

$$
\mathcal{L}=P(X), \quad \text { with } \quad X=\dot{\theta}-\frac{\left(\partial_{i} \theta\right)^{2}}{2 m}-m \Phi
$$

where $\Phi$ is the gravitational potential. This is going to be the case studied in the next section for the DM superfluid.

With that Lagrangian we are able to describe the theory as the other approaches we used to describe the BEC and superfluid theories.

\section{Equivalence of the EFT description:}

For low energies, and in the non-relativistic case, the EFT of superfluids is equivalent to the microscopic description presented above. Considering $P(X) \propto(\dot{\theta} / m)^{n}$.

$$
\begin{aligned}
& 2-\operatorname{body} \mathcal{L}=-|\partial \Psi|^{2}-m^{2}|\Psi|^{2}-\frac{g}{2}|\Psi|^{4} \quad \Longleftrightarrow \quad \mathcal{L}=P(X) \propto X^{2} \quad \longrightarrow p \propto \rho^{2} \\
& 3-\operatorname{body} \mathcal{L}=-|\partial \Psi|^{2}-m^{2}|\Psi|^{2}-\frac{g_{3}}{2}|\Psi|^{6} \quad \Longleftrightarrow \quad \mathcal{L}=P(X) \propto X^{3 / 2} \longrightarrow p \propto \rho^{3}
\end{aligned}
$$




\section{Superfluid hydrodynamics}

From this formalism, we can also describe the superfluid hydrodynamics. From (69), we re-write the field equation with respect to the number density to obtain the continuity equation:

$$
\dot{n}+\frac{1}{m} \partial_{i}\left(n \partial_{i} \pi\right)=0 .
$$

The gradient of the field $\pi$ can define the velocity of the superfluid, $\mathbf{v}_{\mathrm{S}}=-\nabla \theta / m=$ $\nabla \pi / m$, we can derive the second equation of superfluid hydrodynamics:

$$
\dot{\pi}=-\mu(n)-\frac{m v_{\mathrm{s}}^{2}}{2} .
$$

\section{Validity of the EFT}

As we saw above, this theory is valid for low energies, or long wavelength, and breaks for high energies. The Lagrangian shown here is valid in leading order in derivative expansion. In this regime, it reproduces the results from hydrodynamics of superfluids. But this framework also allows us to go beyond leading order in this momentum expansion, the next-to-leading order (NLO) Lagrangian. In Son and Wingate (2006), they show a prescription to take into considerations next-to-leading order terms. This can be done in this framework at arbitrary order, only requiring that the NLO Lagrangian respects the symmetry of the system, and at the cost of introducing new free parameters. This might allow the study of those systems in a regime that is challenging for the microscopic perturbative description.

However, the validity of this effective theory constructed here still needs to be checked as higher order terms in the Lagrangian can only be neglected if the sound speed of the theory is not too small. In Sect. 4.2.5, we describe the validity of the EFT for that concrete example of superfluid and show that the theory is valid for the parameters of the model.

This theory is also only valid in the absence of dissipation. A discussion of how to describe dissipative phenomenon in this EFT approach can be seen in Berezhiani (2020).

The formalism of the EFT presented here is used to describe the low-energy dynamics of the superfluid Goldstone mode, the phonon. This formalism is more general, tough, and translates into an EFT language the hydrodynamics of fluids at zero temperature and without dissipation, so it can be generalized to describe superfluids with different equation of state, superconductivity, unitary Fermi gas, among other systems. This low-energy EFT approach is very useful to describe the dynamics of various physical systems and writing the superfluid in this macroscopic effective Lagrangian offers us the chance to study the dynamics of this system without having to work out the details coming from the microscopic short distance physics. With that, this formalism allows us to study the behaviour of more complicated superfluids, with different equations of state that might come from different and more complicated interactions. This will be useful to describe the DM superfluid model, in Sect. 4. 


\subsection{Rotating superfluid-quantum vortices}

When we rotate a normal fluid, the fluid rotates together with the recipient in a homogeneous way, like a rigid body, with vorticity $\nabla \times \mathbf{v}_{\mathbf{n}}=\nabla \times \times \mathbf{r} \neq 0$, where the normal fluid velocity $\mathbf{v}_{\mathbf{n}}$ of a rotational fluid is given by the cross product of the angular velocity and the position.

As we saw above, a superfluid, described by a weakly interacting BEC, has irrotational flow: $\mathbf{v}_{\mathbf{s}}=(\hbar / m) \nabla \theta$ which gives $\nabla \times \mathbf{v}=0$, where viscosity is absent. This is the defining property of a superfluid. In another language, this means that the circulation around a closed contour $\mathcal{C}$ in a superfluid is given by

$$
\Gamma=\oint_{\mathcal{C}} \mathbf{v} \cdot \mathrm{d} \mathbf{l}=\frac{\hbar}{m} \oint_{\mathcal{C}} \nabla \theta \cdot \mathrm{d} \mathbf{l}=\frac{\hbar}{m} \int \nabla \times \nabla \theta=0,
$$

where $\mathrm{dl}$ is a length element on the path $\mathcal{C}$ and $A$ is the area enclosed by this contour. When a superfluid is rotated, this property says that the superfluid would not rotate, but would remain stationary. So, how can we rotate a superfluid and maintain the irrotational flow? This is possible if the superfluid phase presents a singularity.

A superfluid is a state where the system is described by one macroscopic wavefunction. In the presence of this singularity, this wavefunction is single valued, $\psi(\theta)=\sqrt{\rho / m} e^{i \theta}=\psi(\theta+2 \pi n)$, which leads to the quantization of the circulation:

$$
\Gamma=\oint_{\mathcal{C}} \nabla \theta \cdot d \mathbf{l}=\frac{\hbar}{m} \Delta \theta=2 \pi n \frac{\hbar}{m} .
$$

This property above describes a vortex. The way a superfluid rotates is inhomogeneous by forming quantized vortices (Tsubota et al. 2013a, b; Barenghi et al. 2001).

From that we can see that the azimuthal velocity of a irrotational fluid is given by $v_{\phi}=n(\hbar / m)(1 / r)$, where $r$ is the distance to the center of the closed loop $\mathcal{C}$ and $\phi$ is the azimuthal angle. At the center, we have the vortex core, as $\lim _{r \rightarrow 0} \psi \rightarrow 0$, of size equal to the healing length, where the density $\rho$ vanishes and $\theta$, the phase, rotates by $2 \pi$ around the core. The flow in the center is given by the vortex line. The vorticity of the rotating superfluid is given by

$$
\nabla \times \mathbf{v}_{s}=\frac{\hbar}{m} \sum_{i=1}^{N_{v}} 2 \pi \delta\left(\mathbf{r}_{i}\right) \hat{\mathbf{z}}
$$

where $\mathbf{r}_{i}$ is the location of the $N_{v}$ vortices, and we considered that the vortex lines are in the $z$-direction. The vorticity is non-zero only at each vortex. So the flow is irrotational in most of the superfluid, except in the vortices. Given that the vortices are line singularities, they form a lattice of uniformly distributed vortices in the superfluid and carry the angular momentum of the rotation $L_{z}=n L_{q m}$, where $L_{\mathrm{qm}}=\hbar N$ is the minimum angular momentum necessary to have one quantized vortex and $N=$ $\int|\psi|^{2}$ is the number of particles in the condensate. This configuration is energetically 

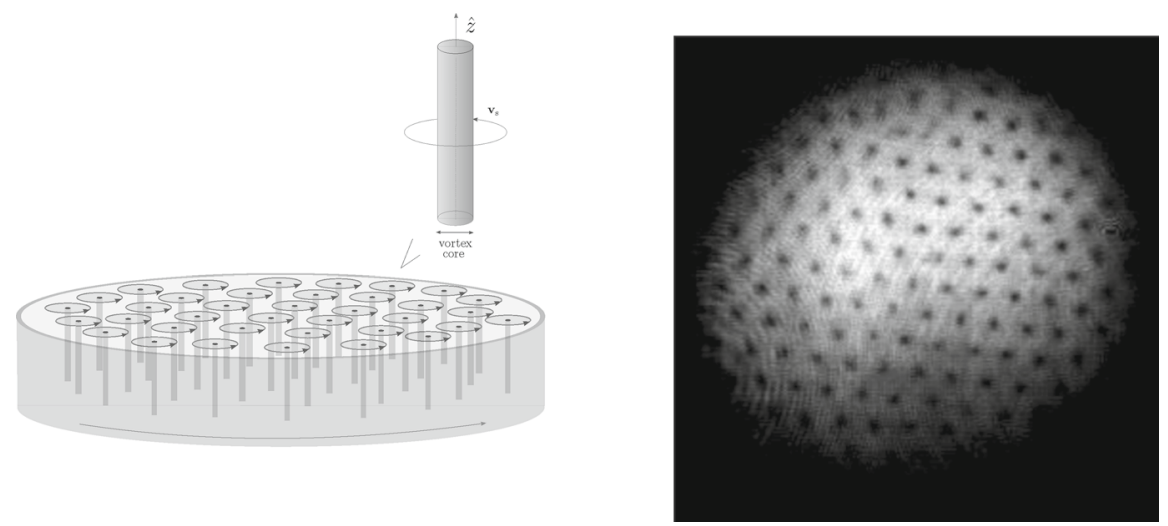

Fig. 8 Left panel: schematic of a superfluid in a rotating box. A lattice of vortices is homogeneously formed in the superfluid. Right panel: the experimental observation of a highly ordered vortex lattice. Image reproduced with permission from Abo-Shaeer et al. (2001), copyright by AAAS

preferable (instead of for example concentric sheets around the superfluid). With that, the spatially averaged vorticity is given by

$$
\left\langle\nabla \times \mathbf{v}_{s}\right\rangle=\frac{\hbar}{m} n_{v} \hat{\mathbf{z}}=2 \Omega \hat{\mathbf{z}}
$$

where $n_{v}=2 \Omega /(\hbar / m)$ is the density of vortices, which is related to the angular velocity (Feynman 1955). Although most of the superfluid has irrotational flow, the rotational flow is given by the vortices in a way that the whole superfluid then effectively flows as a normal fluid $\left\langle\mathbf{v}_{s}\right\rangle=\mathbf{v}_{n}$, allowing the superfluid to rotate.

Given that the superfluid has irrotational flow, if it is rotated by a small $\Omega$, the superfluid will remain stationary. There is a critical angular velocity, $\Omega_{\mathrm{c}}$, above which the nucleation of vortices in the superfluid occurs. The critical velocity is given by Guadagnini (2017):

$$
\Omega_{c}=\frac{1}{m R^{2}} \ln \left(\frac{R}{\xi}\right),
$$

where $R$ is the radius of the cylinder where the superfluid is contained. Through the dependence in the healing length we can see that the critical angular velocity depends on the interaction, for the case of a weakly interacting BEC that behaves as a superfluid. The case where the interaction is attractive, $g<0$, it is known not to produce vortices. This is also the case where the BEC has a finite size coherence, producing only smaller soliton cores. This condition for the formation of cores can also be re-written as $L \gg L_{\mathrm{qm}}$, where $L$ is the angular momentum of the applied rotation to the system.

If there is the formation of vortices, the area density of vortices is given by $n_{\mathrm{v}}=$ $n / A_{\mathrm{BEC}}=m \Omega / \pi \hbar$. From that, we can calculate the size of the vortices created 
( $\pi R_{\mathrm{v}}^{2}=1 / n_{\mathrm{v}}$ ) which depends on the mass and the angular velocity of the fluid, $R_{\mathrm{v}}=\sqrt{\hbar / m \Omega}$.

But what exactly is the structure of these vortices and how are they described? As we saw before, we defined the vortex as a singularity, where the wavefunction is zero at the vortex line, with a quantized circular irrotational flow around the vortex line. Given this, we represent the vortex as an object in cylindrical coordinates: $\psi(\mathbf{r})=f(r, z) e^{i n \phi}$. To describe the density distribution, their structure and size, one has to solve the GrossPitaevskii equation for this object, which in the case of the weakly interacting Bose gas is

$$
-\frac{\hbar^{2}}{2 m}\left[\frac{1}{r} \frac{\partial}{\partial r}\left(r \frac{\partial f}{\partial r}\right)+\frac{\partial^{2} f}{\partial r^{2}}\right]+\frac{\hbar^{2}}{2 m} \frac{n^{2}}{r^{2}} f+V(r, z) f+\frac{g}{8 m^{2}} f^{3}=\mu f
$$

In the limit $n=0$, we recover the standard Gross-Pitaevskii equation. So the term that contains $n$ is called the centrifugal barrier and it is the kinetic energy term from the azimuthal velocity of the vortex. Solving this equation gives the density profile of the vortex, from which we can determine the size, density and general structure of the vortex.

The presence of a trapping or external potential, changes the density of the condensate which also changes the dynamics of the vortices. For details of the formation of vortices in the presence of a trapping potential, see also Fetter (2009).

Since its discovery in Helium 4 superfluid, vortices have been one of the central topics in the research of superfluidity (Madison et al. 2000). They have been experimentally observed in many systems like Helium 4 and Helium 3, superconductors and atomic BECs, including multi-component BECs. These new observational advances allowed us to visualize and study those vortices, as it can be seen in the left panel of Fig. 8.

\subsection{BEC in wave turbulence-kinetic theory}

We studied in this section, the BEC and the superfluid, and showed that their dynamics is described by the Gross-Pitaevskii equation, which is a non-linear Schrödinger equation. This equation expresses the evolution of the condensate, which is described by the collective wavefunction formed by the macroscopic occupation of the ground state by the bosons, in the mean field self-potential. This theory is capable of describing many different condensate systems and describe the properties of BECs that can be tested with experiments. There is, however, an alternative way to describe the physics of a BEC, using the theory of wave turbulence.

Wave turbulence is the theory that describes non-equilibrium statistical systems using random non-linear interacting waves. These random interacting waves are the fundamental constituent of the theory. Waves in a non-linear medium interact and behave very differently than waves in vacuum and wave turbulence describes these systems of interacting non-linear waves. This approach can be used to describe many different physical system from quantum fluids to astrophysics, which is our main interest. Wave turbulence arises in classical context in systems like non-linear optics, 
surface waves in water, magnetic turbulence in interstellar gases, among many others (see Nazarenko 2011a for a list of examples and references). Quantum fluids are also physical systems where wave turbulence occurs, and this approach can be used to explain exotic superfluids, atomic BEC, second sound waves in ${ }^{4} \mathrm{He}$ superfluid, and other systems as it can be seen in Kolmakov et al. (2014). Wave turbulence is a formalism specially useful to model these systems numerically and for experimental studies.

Wave turbulence can arise either in strong or weak interacting system of random non-linear waves. Weak turbulence is a limit where it is considered that all the waves are weak and have random phases. It provides a theoretical framework for wave turbulence theory to describe many physical system, representing those systems by a wave kinetic equation. This equation describes the evolution of the wave spectrum, two point correlation function or the probability distribution function, via averaging over random phases, of a system. This kinetic equation is different for each system and can have a three-wave non-linear interactions, four-wave interactions or even higher order depending on the system described. Higher moments of this equation allow the study of deviations of Gaussianity or to explore the limits of validity of the wave turbulence.

Wave turbulence can also be used to describe Bose-Einstein condensates and superfluids. It can offer a way of describing different stages of a BEC systems in a nearly equivalent way of its proper description through the non-linear Schrödinger equation presented above. We are going to show now how and for which limits a BEC can be described using wave turbulence approach. We are going to show that wave turbulence with four wave interaction, which reproduces the non-linear Schrödinger equation, can only describe the classical initial evolution of a BEC, breaking as the condensate evolves. We also show that the quantum evolution of a condensate can be described by this theory. Wave turbulence also offers a description for a BEC and superfluids outside the weak interaction limit, but we are not going to discuss this case here.

We want to develop a statistical description of the wavefunction and of the nonlinear Schrödinger equation, and determine the kinetic equation which is the main equation of the wave turbulence theory. This is done by describing the evolution of an ensemble of waves (in contrast to the Boltzmann equation that is the equation that describes the evolution of a distribution of particles). For that we introduce the Wigner distribution (Wigner 1932)

$$
f_{\mathbf{p}}(\mathbf{x}, t) \equiv \int d \mathbf{y} e^{-i \mathbf{p} \cdot \mathbf{y}}\left\langle\psi\left(\mathbf{x}+\frac{\mathbf{y}}{2}\right) \psi^{*}\left(\mathbf{x}-\frac{\mathbf{y}}{2}\right)\right\rangle,
$$

where the average is the average with respect to the random initial phases of the field. The Wigner distribution is a quasi-probability distribution, it behaves like a probability distribution but it does not obey all the axioms of probability theory by Kolmogorov (Kolmogorov 1933). It can be used to represent this ensemble of waves with random initial phases, or of random classical fields. As a quasi-probability, it can acquire negative values indicating interference of waves in phase-space (Choi 2006) describing the undulatory behaviour that can be captured in this description. 
We now want to describe the evolution of this ensemble. We want to have a kinetic theory that is nearly equivalent to the GP equation (25) for a weakly interacting system. For that, as we can see from the interacting Hamiltonian of this theory (20), we need a four wave interaction. With that we can rewrite (25) using (82) as

$$
\frac{\partial f}{\partial t}+\frac{\mathbf{p}}{m} \cdot \nabla_{x} f=2 \operatorname{Im} \int \mathrm{d} \mathbf{y} e^{-i \mathbf{p} \cdot \mathbf{y}}\left\langle\psi\left(\mathbf{x}+\frac{\mathbf{y}}{2}\right) \psi^{*}\left(\mathbf{x}-\frac{\mathbf{y}}{2}\right) U_{\text {tot }}\left(\mathbf{x}+\frac{\mathbf{y}}{2}\right)\right\rangle,
$$

where we wrote $f_{\mathbf{p}}(\mathbf{x}, t)$ as $f$ for simplicity of notation. Here we are considering that $U_{\text {tot }}=g|\phi(x)|^{2}$. This is the kinetic equation or the wave kinetic equation.

This equation gives the evolution of the distribution that describes the an ensemble of waves that is the two point correlation function of the field. This distribution can be interpreted as the wave action or the particle number density or particle occupation number, where this second interpretation leads us to the idea we had before that $\psi$ can be thought as the classical limit for the quantum wavefunction or field of a weakly interaction Bose gas (Dyachenko et al. 1992; Mendonça et al. 2005).

We want to obtain the closed form of the kinetic equation, expressed in terms of $f$. The above kinetic equation presents, in the case of the four wave interaction, a four point correlation function. For the case of weak coupling that we are studying here, the Wick theorem is approximately valid and we can express this four-point function into the sum of two-point functions, reducing the higher order problem into a lower order one:

$$
\left\langle\psi_{1} \psi_{2}^{*} \psi_{3} \psi_{4}^{*}\right\rangle=\left\langle\psi_{1} \psi_{2}^{*}\right\rangle\left\langle\psi_{3} \psi_{4}^{*}\right\rangle+\left\langle\psi_{1} \psi_{4}^{*}\right\rangle\left\langle\psi_{3} \psi_{2}^{*}\right\rangle+\left\langle\psi_{1} \psi_{2}^{*} \psi_{3} \psi_{4}^{*}\right\rangle_{\mathrm{conn}}
$$

where we simplified the notation writing $\psi_{i}=\psi\left(x_{i}\right)$, and the last term represents the connected part of the correlation function, the non-diagonal part of the operator. The connected part is non-zero if the distribution is non-Gaussian, and it is also higher order in the interaction.

In the regime of small non-linearities and taking the random phase approximation (Navez 2005), which allows us to ignore the higher order correlations, we can write the closed form for this equation. We wish to work in Fourier space, so we take the Fourier transform of the field. The above approximations correspond to ignoring higher Fourier moments and writing Eq. (83) in terms of the two-point correlation function $\left\langle\psi_{k_{i}} \psi_{k_{j}}\right\rangle=n\left(k_{i}\right) \delta\left(k_{i}-k_{j}\right)$, where $\delta\left(k_{i}-k_{j}\right)$ is the Dirac delta (Nazarenko and Onorato 2006; Nazarenko 2011b; Zakharov et al. 1992). Given these assumptions, we can re-write the kinetic equation in closed form:

$\dot{n}_{k}=4 \pi \int n_{1} n_{2} n_{3} n_{4}\left[\frac{1}{n_{k}}+\frac{1}{n_{3}}-\frac{1}{n_{1}}-\frac{1}{n_{2}}\right] \delta\left(\mathbf{k}+\mathbf{k}_{3}-\mathbf{k}_{1}-\mathbf{k}_{2}\right) \delta\left(k^{2}+k_{3}^{2}-k_{1}^{2}-k_{2}^{2}\right) d \mathbf{k}_{1} d \mathbf{k}_{2} d \mathbf{k}_{3}$.

The above equation is the equation for the evolution of the wave spectrum. One can also write the equation for the higher moments if interested in investigating deviations from Gaussianity or the limits where this description breaks, among other phenomena.

This equation reproduces the non-linear Schrödinger equation, which is the equation that governs the evolution of a wavefunction. Any wavefunction. It can be thought as 
the quasi-classical limit at high occupation number of the quantum kinetic equation (we discuss this later in this section). This equation describes the evolution of any ensemble of classical waves that is described by the Schrödinger equation. It describes a noncondensed system since in this description condensate density, which is the density of particles in the ground state which in the language of waves translates into waves with $k=0$, is relatively small (in comparison to a strong BEC deep into the region $T \ll T_{\text {cr }}$, where the number of particles in the ground state is almost equal to the total number of particles). When the condensate density is large or the population of lowest momentum states is large, the system is not weakly non-linear anymore and the above description breaks. However, this description can be used to the initial stages of a condensate, when the condensate density is still small. We call this limit "weak" condensate. When we have self-interactions the system can form a superfluid, and for this four-wave system this equation describes the vicinity of superfluid transition. Therefore, the kinetic equation with four-wave interaction can describe the classical initial evolution of a BEC, when we have a "weak" condensate.

As temperatures drop and the condensate density becomes larger, we have a "strong" condensate. In this stage of the evolution of the condensate the above description with a four wave interaction is not a description of the system, and the evolution of the occupation number of the condensate needs to be described by the wave kinetic equation with three-wave interaction (Nazarenko 2011b; Zakharov et al. 1992; Dyachenko et al. 1992; Lvov et al. 2003). This three-wave interaction representation describes the later phase in the evolution when the condensate is strong and fluctuations on top of the condensate are only given by phonons. Only at this stage the theory can again be described by weak turbulence, but now involving three waves. The intermediary regime between these two description is more complicated. After the four-wave description breaks down, the system is highly non-linear composed by a gas of hydrodynamical vortices. Only after these vortices annihilate and most of the systems are in the condensed phase, one can use the three-wave description.

One possible solution of (85) is the Kolmogorov-Zakharov (KZ) spectra, which is a non-equilibrium steady-state solution. Within this solution, we can have turbulent cascade processes, with a dual cascade for different direction of the energy flux. The interpretation of these dual cascade processes in $\mathrm{BEC}$ is interesting and corresponds to techniques used in experimental realizations of BEC. Inverse cascading, which is the non-equilibrium transfer of particle to the lowest energy momentum can be though as condensation. The initial process of BEC formation can be achieved in this process as a non-equilibrium condensation. The forward cascading is a processes is the energy transfer to higher momentum states, higher energy level. When the condensate is in a trap, these particles are going to leave the trap, and this is called evaporative cooling.

We studied above the case without a trapping potential. When in a trapping potential, the condensate density is now coordinate dependent and the behaviour in this trap will depend on the relation between the characteristic mean free path of the excitation wave packets and the size of the trap or the range of the force that produces this potential.

We are gong to see in Sect. 4.1.4 another solution of the above four-wave kinetic equation in the case of long-range interactions, like the gravitational interaction. The potential is present and it is the gravitational potential. This is described by the Landau kinetic theory, and can describe the initial stages of a condensate in the presence of 
gravitational interactions. Different from the KZ description of condensation discussed above, in this case, condensation does not arise from a cascading process but from a dissipation process.

We described above the four-wave classical kinetic equation. This is a good classical limit representation of the initial stages of a BEC. However, this is not a description of strong condensates, as a three wave kinetic equation needs to be adopted instead. This is also not a description of the quantum condensate. Wave turbulence theory can also be used to describe the full quantum regime of condensate, and not only the classical limit described until now. To be able to do that we first need to discuss the statistical distributions that the kinetic equations describe.

One of the distributions that is a solution of the classical equation (85) is the Rayleigh-Jeans (RJ) distribution, $n_{k}=T /\left(k^{2}+m u\right)$, with $T>0$ and $\mu>0$. This is similar to what we find when we are studying classical limit of the systems described by the Gross-Pitaevskii equation. Thu full quantum system described by (21) has a occupation number that describes a Bose-Einstein statistics. The classical limit of this system, described by the Gross-Pitaevskii equation has a mean occupation number in equilibrium described by the RJ distribution, the classical limit of the Bose-Einstein distribution: $\left\langle n_{k}\right\rangle=k_{B} T /\left(\epsilon_{k}-\mu\right)$, where $\mu<0$. This system describes a condensate only when the occupation of the ground state is macroscopic, $\left\langle n_{k}\right\rangle \rightarrow\left\langle N_{0}\right\rangle$.

The temperature $T$ in this RJ distribution is related to the initial energy of the system $E_{0}=\int \mathrm{d} \mathbf{k} \omega_{k} n_{k}=T \int \mathrm{d} \mathbf{k}$, where $\omega_{k}=k^{2}+\mu$, in connection to thermodynamics. From this, we can see that since each degree of freedom of the theory has the same energy $T$, for a continuous and infinite system, the this and the energy diverge. This is the classical RJ catastrophe or the UV catastrophe.

Thus, the UV catastrophe is inherent to the ensemble of classical nonlinear waves. It is argued then that for the RJ solution to be a relevant solution of the kinetic equation, a cutoff needs to be introduced to regularize the UV catastrophe. This is as a phenomenological way of making the classical description of the system valid. Therefore, to describe a BEC in such a classical theory one needs to have a momentum cutoff in the theory. This truncated system then can be used to describe the evolution of a classical ensemble of waves via the kinetic theory derived above. In realistic systems, this cutoff sometimes comes naturally from dissipation or limits of the simulation or experiment. If one is working in a description where the UV catastrophe does not take place, where the evolution period of the system is not threatened by this divergence, then the above description for the classical condensate also holds. This will be the case of Sect. 4.1.4.

Another solution to make the description valid (avoiding the UV catastrophe) is to go to a quantum statistics. We can modify the kinetic theory to obey a Bose-Einstein statistics. We generalize the kinetic equation to:

$$
\begin{gathered}
\dot{n}_{k}=4 \pi \int n_{1} n_{2} n_{3} n_{4}\left[\left(\frac{1}{n_{k}}+1\right)\left(\frac{1}{n_{3}}+1\right)-\left(\frac{1}{n_{1}}+1\right)\left(\frac{1}{n_{2}}+1\right)\right] \\
\delta\left(\mathbf{k}+\mathbf{k}_{3}-\mathbf{k}_{1}-\mathbf{k}_{2}\right) \delta\left(k^{2}+k_{3}^{2}-k_{1}^{2}-k_{2}^{2}\right) \mathrm{d} \mathbf{k}_{1} \mathrm{dk}_{2} \mathrm{dk}_{3} .
\end{gathered}
$$


The Bose-Einstein statistics is now a solution of this equation. This is the quantum kinetic equation and can be used to describe the full quantum BEC.

We showed above that we can use wave turbulence as an approximate description of some regimes of a BEC. The classical four wave kinetic equation can be used to describe the initial stages of a BEC in the classical limit. Different description will arise for different stages of the evolution of the condensate. Wave turbulence can also describe both classical condensate evolution and quantum evolution. A BEC is a quantum phenomena, but its classical evolution can approximately be described by wave turbulence which is a convenient description, in particular for simulations.

We are going to use kinetic theory to describe the formation of the BEC in Sect. 4.1.4.

\subsection{Summary and discussion: what is a condensate?}

We saw in this section, an introduction to two of the most interesting phenomena in quantum mechanics, the BEC and superfluids. We went through all these concepts in detail with the goal to give a proper definition of Bose-Einstein condensation in the context it is well understood and measured.

BEC is the phenomena of macroscopic occupation of the ground state that happens at low temperatures. BEC is a consequence of the quantum statistics of bosons, and it is an inherently a quantum phenomena. Equivalent to having a macroscopic occupation of the ground state, condensation can be though a the regime where the interparticle distance is smaller than the de Broglie wavelength of the bosons, which leads to a superposition of these wavefuctions, creating a macroscopic wavefunction that describes the condensate, which is a macroscopic quantum object. One of the main properties of a BEC is that it presents macroscopic (long range) quantum coherence.

We also saw that we can describe a more realistic condensation processes using a weakly interacting Bose gas, which exhibits superfluidity upon condensation. This theory is described by the fully quantum many-body Hamiltonian. For a large number of particles $(N \gg 1)$, this Hamiltonian is very complicated to be studied. But, when the interactions in the BEC are weak, the BEC is dilute ${ }^{22} n a^{3} \ll 1$, and for large $\mathrm{N}$, we can take the mean field approximation. In the mean field approximation, we can make the huge simplification that the many-body wavefunction can be approximated by an effective single-particle wavefunction. This means that the wavefunction of the condensate can be written as

$$
\hat{\Psi}(\mathbf{r}, t)=\psi(\mathbf{r}, t)+\delta \hat{\Psi}(\mathbf{r}, t),
$$

where $\psi(\mathbf{r}, t) \equiv\langle\hat{\Psi}(\mathbf{r}, t)\rangle$ (this is a classical quantity, because if it was quantum this average would be zero). The field $\psi(\mathbf{r}, t)$ is the classical field or classical wavenumber. Quantum effects are suppressed in this limit (the depend on $1 / N$ ), and the BEC is well

\footnotetext{
22 The BEC has 3 scales in the absence of a trapping potential: the de Broglie wavelength, the $s$-wave scattering length $a$ and the inter-particle distance $d$. To describe the scattering of two particles that have large $\lambda_{d B}$ as the scattering of two bodies, we have to have $d \gg a$. The mean field approximation is applicable in the limit where we have many particles $N$ large and $n a^{3} \ll 1$, meaning that the interactions in the condensate are weak, which translates to $a \ll \lambda_{d b}$. A condensate that follows this condition is said to be dilute, which means that for fixed $n$, the bosons almost do not interact, $a$ must be very small.
} 
approximated by a classical theory. ${ }^{23}$ The classical field that represents the condensate satisfies the (classical) non-linear Schrödinger equation or Gross-Pitaevskii equation. Therefore, the classical Gross-Pitaevskii or non-linear Schrödinger description of a condensate is a mean field description of condensate.

An important detail about the mean field approximation. In the classical limit of a scalar field we also have (87), where $\psi=\langle\hat{\Psi}\rangle$ is the scalar field and $\delta \hat{\Psi}$ are suppressed quantum corrections. Now, when a condensate can be treated as classical we are also in the limit where we can expand (87), but we have $|\psi(\mathbf{r}, t)|^{2}=n_{0}=n$, which means that there is a macroscopic occupation of the ground state. For the classical limit of the condensate, as the term $\delta \hat{\Psi}(\mathbf{r}, t)$ becomes more and more important and the mean field approximation breaks, this can be seen as depletion of the condensate. This limit can be broken if temperature or interactions are increased, then quantum and thermal fluctuations deplete the condensate. ${ }^{24}$ At this point, the mean field approximation breaks and this classical description of the condensate cannot be used anymore.

Although the condensate to be formed depends on the quantum statistics of bosons and it is a quantum phenomena, it can be treated as classical in the mean field approximation. In an equivalent way, in the field description of BEC, the condensate can be described in the mean field limit by a classical field. ${ }^{25}$

Summarizing A BEC can be described by a coherent classical scalar field that satisfies the Gross-Pitaevskii equation, in the mean field approximation where most of the particles are in the ground state, $|\psi(\mathbf{r}, t)|^{2}=n_{0}=n$.

The term "condensate" is used in the literature loosely meaning different things for different authors. I will use throughout this review the definition presented here. Therefore, every time I am using the term condensate, I am referring to the definition described here.

\section{Ultra-light dark matter}

After the introduction in the previous sections of the concepts that are going to be applied in this part of the review, we are finally ready to discuss the ULDM models.

Ultra-light DM denotes a class of models where DM is composed by ultra-light bosons. These models were introduced as a new class of DM models that can address

\footnotetext{
23 The mean field approximation, $N$ large and weak coupling, and the classical limit $(\hbar \rightarrow 0)$ not always coincide. The mean field approximation is called semi-classical in some places of the literature. In some instances, the mean field $N \rightarrow \infty$ can be recast as a classical limit (Dimonte et al. 2018). The mean field approximation is usually concerned to systems that preserve the number of particles, like condensates.

${ }^{24}$ It is also possible to describe a non-condensate quantum system in a classical limit. This was seen in the wave turbulence theory. In this case, there is no macroscopic occupation of the ground state but the entire system is still in the high occupation classical limit. In this case, $\delta \hat{\Psi}$ is not the depletion from the condensate but it represents the quantum correction to the system.

25 There is only one example in the literature of condensed matter physics where there is classical "condensation". This happens for electromagnetic light waves in nonlinear optics (Sun et al. 2012; Conti 2012). Kinetic condensation is achieved when the light beam goes from a disordered to a coherent state. However, as it was emphasized in Connaughton et al. (2005), this classical condensation is a process analogous to the (genuine) Bose-Einstein condensate, having similar properties and obeying the non-linear Schrödinger equation. This is a very new and active field of study and it is going to be very interested to see the development of this field.
} 
the small scale challenges of $\Lambda \mathrm{CDM}$, but mainly as models that offer a novel and rich phenomenology in galaxies that can be tested with small scale observations. The general idea of those models is that inside a virialized DM halos, ULDM thermalizes and forms gravitationally bounded cores that can be described as a BEC or a superfluid. In this way, these models behave like CDM on large-scales, with modified initial conditions, recovering the incredible observational successes of this description, while inside galaxies they present a wave-like behaviour.

To have this behaviour inside galaxies, the mass of this bosonic DM has to be very small. There are many models in the literature of ULDM that present this wave-like behaviour in galaxies, and the specific range of masses where this wave-like behaviour happens in galaxies depends on the specifics of the models. However, we can estimate in a model-independent way the range of masses of the ULDM particles to present this behaviour in galaxies. The mass of the ULDM has to be:

$$
10^{-25} \mathrm{eV} \lesssim m \lesssim 2 \mathrm{eV}
$$

The lower bound on the mass is very general and comes from the fact that the size of the condensate core cannot be larger than the halo, since we want the condensate only on galactic scales and normal DM on larger scales. The maximum case we can have for the formation of a condensate is where the de Broglie wavelength of the ULDM particle is of the order of the size of the halo. Taking this bound at virialization, there is a maximum value on how large the de Broglie wavelength can be $\lambda_{\mathrm{dB}}<R_{200}$. Taking $z_{\text {vir }} \sim 2$ and for halos with mass of order of $10^{12} M_{\odot}$, we can see that for a spherical halo (Rindler-Daller and Shapiro 2012) this imposes a lower bound on the mass

$$
m>m_{\mathrm{H}} \equiv \frac{2 \hbar}{\sqrt{3 G}}(R M)^{-1 / 2} \approx 10^{-25}\left(\frac{M}{10^{12} M_{\odot}}\right)^{-1 / 2}\left(\frac{R_{200}}{100 \mathrm{kpc}}\right)^{-1 / 2} \mathrm{eV}
$$

We can also impose an upper bound on the mass asking the question: what is the biggest mass I can have that ULDM forms a core inside the galaxy? Again, to answer this question one needs to work with a specific ULDM model to study the Jeans theory of this model and the solutions of the equations in this region, which would give a bound within this model for the creation of these cores. However, one can try to be more general. The non-CDM behaviour happens in the regions where the wave behaviour takes place. So, the interesting non-CDM behaviour occurs on scales of the order or smaller than the scale that characterizes the wave which given by the de Broglie wavelength. This is the maximal case where the de Broglie wavelength of the ULDM particle is of the size of the galaxy. But one can also think that we can obtain a galaxy size wave as the superposition of the de Broglie wavelength of each of the ULDM particles (which are themselves smaller than the galaxy in this hypothesis). Then we can calculate the biggest mass, which means smaller de Broglie wavelength if each particle, for which this superposition yields a galaxy size wave. This translates to the condition that de Broglie wavelength of the boson DM is larger than the inter-particle 
distance between each boson,

$$
\lambda_{\mathrm{dB}} \sim \frac{1}{m v}>l=\left(\frac{m}{\rho}\right)^{1 / 3} \Longrightarrow m<\left(\frac{\rho}{v^{3}}\right)^{1 / 4},
$$

where assuming a spherical halo, the interparticle distance is defined as the radius of a sphere with density $\rho$. This gives a bound on the mass of the DM particle. This condition is the same as the condition that a gas need to have to condensate into a BEC, as we can see in the box in Sect. 3.1 since it is equivalent to the condition of having macroscopic occupation number of the ground state for temperatures below the critical temperature of the system. This condition does not determine condensation of DM in the halo since showing that condensation happens in the halo is much more complicated than this ideal gas condition that is not realistic for the halo, but it is a 0th order condition for this phenomena together with the assumption of thermalization. But here we use it only as a condition to form a galaxy size macroscopic wave from the superposition of the individual particles' waves.

We use the density and velocity of the dark matter halo like described in Sect. 2 from standard spherical collapse (Berezhiani and Khoury 2016), and take this bound at virialization:

$$
\begin{aligned}
& \rho_{200}=200 \rho_{\mathrm{cr}} \sim 1.95 \times 10^{-27}\left(1+z_{\mathrm{vir}}\right)^{3} \mathrm{~g} / \mathrm{cm}^{3}, \\
& V_{200} \sim 85\left(\frac{M}{10^{12} M_{\odot}}\right)^{1 / 3} \sqrt{1+z_{\mathrm{vir}}} \mathrm{km} / \mathrm{s}
\end{aligned}
$$

where we derived these expressions assuming $H_{0} \sim 70 \mathrm{~km} \mathrm{~s}^{-1} \mathrm{Mpc}^{-1}$ and a halo mass of order of the MW. This gives the bound

$$
m \lesssim 2.3\left(1+z_{\mathrm{vir}}\right)^{3 / 8}\left(\frac{M}{10^{12} M_{\odot}}\right)^{-1 / 4} \mathrm{eV}
$$

Taking $z_{\text {vir }} \sim 2$ and for halos with mass of order of $10^{12} M_{\odot}$, we have an upper bound for the mass of the ultra-light DM particle to have galaxy-sized wave coming from the superposition of the wave of each particle: $m \sim 2 \mathrm{eV} .^{26}$

I would like to bring the readers attention to following. The issue of condensation of ULDM in the halo is one of huge debate in the literature and we are going to discuss this in Sect. 4.1.5.

The range of masses showed above is just an estimate of the maximal higher and lower masses that the ULDM can have. Each specific ULDM model has a mass range where this behaviour in galaxies takes place and that it is in agreement with observations which depends on the specifics of the models. However, those bounds have to always fall within this general range (assuming ULDM is all the DM in the universefor studies where this is not true see Hložek et al. 2015, 2018). We will see in Sect. 5 that

\footnotetext{
${ }^{26}$ Remember here what we said before that it is much harder to show condensation for ULDM under the influence of gravity in the halo of a galaxy. Condensation has been shown to happen in the case of the FDM in Levkov et al. (2018), and we discuss this in more detail in Sect. 4.1.4.
} 
for the FDM model, using CMB and LSS observations and assuming again the FDM is all the DM, that the lower bound in the mass is very close and within this theoretical estimation. Now for the DM superfluid model, as we will see in Sect. 4.2, respects the upper bound presented here with the mass of its particle very close to this limit.

The mass range for the ULDM presents masses that are much smaller than the ones usually considered for DM candidates and cannot be produced thermally in the early universe. Therefore, ULDM is a non-thermal relic, having to be created by a nonthermal mechanism to be cold today and behave as DM. There are many non-thermal production mechanism (see 'DM relics' panel below) but since in this review we are being agnostic on the type of particle that consists our ULDM, we are not assuming any creation mechanism, unless we are talking about a specific microscopic candidate.

With that, for a ULDM candidate with a mass in the range (88), we are going to have a non-CDM like behaviour coming from the presence of this core with wavelike behaviour inside the galaxy, while having a CDM like behaviour on large-scales, with different initial conditions resulting from the different mass and dynamics of the specific ULDM model. ULDM is the name used to the collection of models that have the characteristic stated above. There are many realizations of this behaviour which are present in each of the specific ULDM model that yield a different description of $\mathrm{DM}$ in galaxies, and a distinct and rich phenomenology in galaxies. We are now going to see that those models can be classified into three main classes according to their descriptions in galaxies.

\section{Classification of ULDM models}

The idea of having DM condensation on small-scales is not new and has been around for 30 years (Sin 1994; Ji and Sin 1994; Khlopov et al. 1985). For this reason, there are many models in the literature that were developed to describe a DM component with that behaviour on galactic scales. These models receive many names in the literature. They are either models that have a microscopic description or phenomenological models, which allow for the inclusion of different interactions and for a different dynamics to describe the evolution and non-linear structures of DM in the halo, which in turn can lead to distinct and rich astrophysical consequences on small-scales.

One possible model of ULDM is the axion. This model helped to bring a lot of attention in the literature to this class of models. In the case of the axion, we have a model that has a microscopic description and a well-defined cosmology. The QCD axions and general axions can behave like DM in a large range of parameters. This is also the case for axion-like particles (ALPs), which is another microscopic scalar theory that can describe DM. These microscopic theories behave like DM, but only present interesting phenomenology in small-scales for a more limited range of masses. On small-scales, the behaviour of these microscopic scalar theories can coincide with the behaviour of other phenomenological models of ULDM. For all of those, the non-relativistic action that yield a non-linear Schrödinger-Poisson equation.

In this review, we am going to classify the ULDM models according to the description they present on the small-scales, given by their non-relativistic dynamics on those scales. Each of these classes can contain both phenomenological and microscopic models that yield the same non-relativistic model. This classification is instrumental since it elucidates the physics responsible for the non-relativistic evolution and non- 
linear structures that are formed, and separates the different phenomenology each of those classes present.

These different descriptions also yield different conditions for condensation (or if it condenses of not) and formation of the condensate core. Each of these non-relativistic descriptions is going to describe a different phenomena upon condensation, being possible to have either a BEC or a superfluid, the latter in the presence of interactions.

According to this criteria, we classify the ULDM models into three categories (which somehow agrees with what was suggested in Sharma et al. 2019):

Fuzzy dark matter (FDM): The first category is given by a gravitationally bounded scalar field model. It described by a non-linear Shrödinger equation subjected to a gravitational potential, coupled to the Poisson equation (see 'ULDM classes' box). In this model, condensation under the influence of the gravitational potential is achieved in galaxies where the gravitational attraction is counteracted by the quantum pressure. This class of model can be called fuzzy DM since this name is already very well established for these gravitationally bounded BECs. One of its main realizations, which coined the name fuzzy dark matter is presented in Hu et al. (2000a); Hui et al. (2017), where the DM is given by a light particle with $m \sim 10^{-22} \mathrm{eV}$. The FDM model is the ULDM model that was studied the most in the literature both theoretically and numerically. With a particle with this mass, the FDM model is known to be able to solve some of the challenges from small-scales presented above, and to be in agreement with large-scale observations. The mass of this model does not have to necessarily have this value and need to be determined by observations, although this is the value that gives the most appealing modifications on small-scales. Therefore, this model has one free parameter, the mass of the FDM particle $m$ (we are considering in the review the case where all DM is composed of ULDM). Some interesting phenomenology also emerges from this model, as we will discuss in detail in the next subsections, that can be probed by current and future astrophysical observations. This model has also been called in the literature by wave DM, $\psi$ DM, among other names (Sin 1994; Ji and Sin 1994; Seidel and Suen 1990; Widrow and Kaiser 1993; Chan et al. 2018; Guzmán et al. 1999; Matos and Guzmán 2000; Matos et al. 2009).

Axions and ALPs can also be thought to be in this class, since they yield exactly the same physics on small-scales. However, these models are more general and can describe DM for different values of their mass (like the QCD axion that does not produce such structures).

Self interacting FDM (SIFDM): The second category is called self-interacting FDM (SIFDM), but it also receives the names repulsive DM, scalar field DM, fluid dark matter, among others in the literature (Goodman 2000; Peebles 2000; Silverman and Mallett 2002; Arbey et al. 2003; Boehmer and Harko 2007; Lee 2009; Lee and Lim 2010; Harko 2011; Slepian and Goodman 2012; Dwornik et al. 2014; Guzmán et al. 2014; Harko 2014; Fan 2016; Guth et al. 2015; Chavanis 2018, 2016; Dev et al. 2017; Chavanis 2011; Chavanis and Delfini 2011; Chavanis 2012; Rindler-Daller and Shapiro 2012). In these models, DM is described by a scalar field model, in the presence of gravity, with a (usually) two-body self-interaction. The presence of the interaction makes this model present superfluidity upon condensation. This case is described by the interacting BEC presented in Sect. 3.3, which is the simplest example of a superfluid. The presence of the interaction controls the stability of the core and for 
this reason this model presents a different phenomenology depending not only on the mass of the particle, as for FDM, but given the strength and sign of the interaction. For a repulsive interaction, the condensate has a long range coherence and presents superfluidity. The 2-body case is characterized by having an equation of state (EoS), $P \sim n^{2}$, as we saw in the previous section. Higher order interactions describe SIFDM with different equations of state.

DM superfluid: The third category is called DM Superfluid (Berezhiani and Khoury 2015, 2016; Khoury 2016; Hodson et al. 2017; Berezhiani et al. 2018). This theory was proposed with the goal of reproducing the MOND empirical law on small-scales, presenting a natural framework for the emergence of this theory. Different from the case of SIFDM, to reproduce MOND on small-scales, it requires that the EoS is given by $P \sim n^{3}$, like what is expected by MOND, with a more intricate dynamics describing the small-scales. To accomplish that, this model is described using the EFT of superfluids which allows us to describe superfluids with a more general dynamics and EoS.

\section{ULDM classes}

Classification is based on the different ways they achieve condensation.

Fuzzy DM (FDM) described by a ultra-light scalar field under the influence of gravitational potential. Forms a BEC on galactic scales.

$$
i \dot{\psi}=-\frac{1}{2 m} \nabla^{2} \psi+V_{\text {grav }}
$$

Self-Interacting FDM described by a self-interacting scalar field with 2-body (or higher) interaction. (SIFDM)

$$
i \dot{\psi}=-\frac{1}{2 m} \nabla^{2} \psi+V_{\text {grav }}+g|\psi|^{2} \psi+g_{3}|\psi|^{4} \psi+\cdots
$$

(Superfluid)

$\begin{array}{lccccc}\text { DM } & \text { Superfluid } & \text { described by } & \text { a superfluid with } \\ \text { specific } & \text { EoS } & \text { to } & \text { reproduce } & \text { MOND } & \text { in galaxies. }\end{array}$

(Superfluid)

$$
\mathcal{L}=P(X)
$$

There are many amazing reviews in the literature that focus in different parts of the ULDM class of model, either focusing in microscopic models, like describing axions Sikivie (2008); Arvanitaki et al. (2010); Wantz and Shellard (2010); Kim and Carosi (2010); Kawasaki and Nakayama (2013); Marsh (2016a) or ALPs (Ringwald 2014; Arias et al. 2012; Graham et al. 2015a; Marsh 2018; Niemeyer 2019; Powell 2016), or focusing in one of the classes like the FDM Hui et al. (2017); Suárez et al. (2014); Ureña-López (2019), for which the axions and ALPs describe the same non-linear 


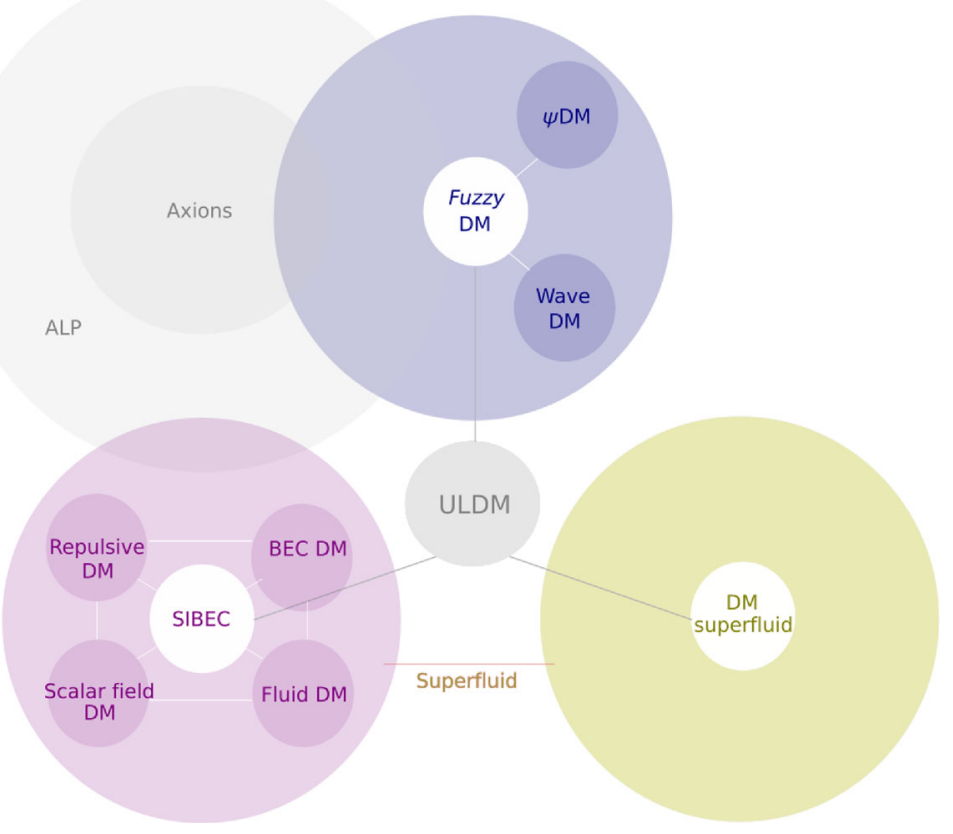

Fig. 9 Map of the ULDM classes of models

theory (given by a Schrödinger-Poisson system in the absence of self interactions). This review follows a classification between the different classes according to their non-relativistic description and includes not only the FDM, but also the other classes of ULDM.

Given that, in this review, we are going to be describing the dynamics each of these classes of models present on small-scales, and the different cosmological and astrophysical consequences this new phenomenology brings. The exception is in the case of the FDM where we also are going to talk a little bit about the cosmology of the axions and ALPs. However, in general, we are going to remain agnostic about the origin of this field and we are going to work out only the gravitational consequences they have. ${ }^{27}$ Understanding these consequences will prepare the field for the next section, where we discuss constraints on these models. We briefly discuss in the end of this section the big role that simulations have in studying these models and also the regimes where these ultra-light fields can behave as dark energy.

\footnotetext{
27 The list of models we present here that compose each of the classes is not completely exhaustive and it only aims to show the diversity of models in the literature, and the different mechanisms they describe. However, all the possible dynamics present on small-scales coming from ULDM are described here and can be within one of these three classes. In Lee (2018) one can find a more complete list of references.
} 


\section{DM relics}

Dark matter candidates can have distinct formation mechanisms, with the main ones being thermal or non-thermal relics produced in the early universe. Depending on this mechanism, different masses and couplings for these are allowed as the correct relic abundance of DM is obtained.

Thermal relics: $\quad$ This refers to the particles, including DM, that are produced from the hot and high density thermal bath of the photon-baryon plasma in the universe. Initially, in the early universe, the universe was in a state where it was hot and dense where particle and photons were very close to thermal and chemical equilibrium. This means that the time scale of the particle interactions $(1 / \Gamma)$ in the plasma are much bigger than the expansion time of the universe $H \ll \Gamma=n\langle\sigma v\rangle$, where $\langle\sigma v\rangle$ is the thermally averaged cross section (Kolb and Turner 1990). As evolution follows and those quantities redshift, at some point $H \sim \Gamma$, and the particle decouples from the thermal bath (at the temperature $T_{\mathrm{fo}}$ ). This is a simplified description of the process called freezeout or decoupling. Depending on the interaction rate of each particles, they decouple at different times. This process is described by the Boltzmann equation and it is how electrons, neutrons and neutrinos are formed. If $T_{\text {fo }} \ll m$, where $m$ is the mass of the particle, the particle decouples as a non-relativistic particle, and it is called a cold relic; otherwise, if $T_{\text {fo }} \gg m$ or $T_{\text {fo }} \sim m$, and we have hot and warm relics.

We can assume that DM is a component that was in contact with the thermal bath and it is a particle produced through decoupling from the thermal bath like described above. For the thermal relics, the particle with smaller mass is hotter. For cold relics, in the case of WIMP created through this mechanism, for the WIMP to have the correct abundance of DM today, the averaged cross section is roughly $\sigma_{\mathrm{DM}-\mathrm{DM}} \simeq 10^{-8} \mathrm{GeV}^{-2}$. This is of the same order of magnitude of the electroweak cross section: $\sigma_{\text {weak }} \simeq \alpha^{2} / m_{\text {weak }}^{2}$ with $\alpha \simeq \mathcal{O}(0.01)$ and $m_{\text {weak }} \simeq \mathcal{O}(100 \mathrm{GeV})$ (Kolb and Turner 1990). Many models of DM are produced thermally like supersymmetric candidates, more complicated WIMP candidates or particle DM decays ${ }^{28}$.

Non-thermal relics: As we saw above, there is a limit for the mass of the DM particle that can be created thermally. The only way of having smaller mass candidates of DM is by having a non-thermal mechanism to produce those DM particles. There are many mechanisms that can produce non-thermal DM candidates that include decaying from topological defects (see for example Sikivie 2008; Hiramatsu et al. 2012 where axions are produced from the decay of axion strings or domain walls), decaying from a massive parent particle, vacuum misalignment, among others (Marsh 2016a; Bhupal Dev et al. 2014). Vacuum misalignment or vacuum displacement is one of the genesis mechanism for these ULDM and axions. The vacuum displacement mecha- 
nism (Preskill et al. 1983; Abbott and Sikivie 1983; Dine and Fischler 1983; Carroll 1999) can be described, in a concise way in the following way. A massive scalar field in an FRW universe, when $H>m_{\varphi}$, is overdamped and it behaves nearly as a constant. So, if we consider that initially this field was displaced from its minimum, $\varphi=\varphi^{*}$, the field has a potential energy given by $\varphi^{*}$. When $H \sim m_{\varphi}$, the field starts to evolve and begins to oscillate in its potential, and in turn redshifts like matter. The mass and the initial displacement fix the energy density of this misalignment field. If we consider that the ultra-light particles are created by this mechanism, it imposes a lower bound on the mass $H\left(a_{\mathrm{eq}}\right) \approx 10^{-28} \mathrm{eV}$, in order to start behaving like DM around equality. For more detail on this mechanism for axions, see Marsh (2016a).

\subsection{FDM and SIFDM}

In this section, we are going to describe the FDM and the SIFDM models. Although they both describe different non-relativistic dynamics and structure formation in the halo, and according to our classification are in different classes, we describe them in this section together since they can be described by a relativistic action. Even tough we are interested in the non-CDM phenomenology of these models on small-scales, these models can also modify the initial conditions for the evolution of the matter perturbations, and depending on the mass, modify the evolution of the model. In this way, the relativistic theory allows us to study the cosmology of this model and with that make predictions that can be tested by large-scale observations like CMB and LSS. This helps us to describe the model in different scales and use observations from large and small-scales to constraint the parameters of the model.

The ULDM is described as a very light scalar field minimally coupled to gravity given by the action,

$$
S=S_{\mathrm{EH}}+S_{\phi}=\int \mathrm{d}^{4} x \sqrt{-\bar{g}}\left[\frac{R}{16 \pi G}+\frac{1}{2} g^{\mu \nu} \partial_{\mu} \phi \partial_{\nu} \phi-\frac{1}{2} m^{2} \phi^{2}-\frac{g}{4 !} \phi^{4}\right],
$$

where $S_{\mathrm{EH}}$ is the Einstein-Hilbert metric, $R$ is the Ricci scalar, $g_{\mu \nu}$ is the metric, $\bar{g}$ is the determinant of the metric and $g$ is self-interaction coupling.

The axion or ALPs are described by an action like this. In this case, this action has a microscopic theory behind it. This potential comes from non-perturbative effects in QCD, for the axion, or other concrete string theory models, and gives a small mass for the axion or ALP. Since in these cases this action comes from a well-defined microscopic theory, the parameters relate to scales from this theory and the mechanisms that originated this particle. There is only a range in the parameter space where the ALPs behave like DM, and that also gives an interesting modification of structure

\footnotetext{
${ }^{28}$ Even the axion has a thermal production channel, if the axion is in contact with the thermal bath, with this axion being hot and contributing to a fraction of the effective number of neutrinos (see Marsh 2016a for a review on that topic).
} 
formation. This happens for masses around $m \sim 10^{-22}-10^{-20} \mathrm{eV}$, which is similar to the range of masses for the FDM model.

But this relativistic action could also be phenomenological that allows the coupling to have a different sign and values, different from the axion case. We are going to see soon that the attractive and repulsive interactions yield different phenomenologies, with the repulsive allowing for much bigger collapse cores.

In this section, we are going to explore the cosmological consequences of this relativistic action, and after go to the non-relativistic regime to study the structure formation in the FDM and SIFDM models and describe the condensate formed in the center of the galaxy. To explore cosmological evolution, we are first going to study the concrete case where this action is the action for the ALPs. We also comment a little on the case of axion. This is very useful since in this case when we have a microscopic theory, we can identify the scale of the parameter and their relations, determine the initial conditions and the DM abundance.

\subsubsection{Formation: ALPs}

We are going to briefly describe here the formation mechanism for ALPs (see Marsh 2016a for a more complete description).

An ALP is a pseudo-Nambu-Goldstone resulting from the spontaneous symmetry breaking of a global $U(1)$ symmetry described by the complex scalar field

$$
\Psi=v e^{i \phi / f_{a}},
$$

where $f_{a}$ is the scale of the spontaneous symmetry breaking and $\theta_{a}=\phi / f_{a}$ is the misalignment angle. In the case of the QCD axion, the symmetry broken is the chiral global $U(1)_{P Q}$, the Peccei-Quinn symmetry, and the introduction of this Goldstone boson solves the strong $\mathrm{CP}$ problem. When the symmetry is broken, the massive radial component $v$ is fixed at the vacuum expectation value (vev), $v_{0, s s b}=f_{a} / \sqrt{2}$, making the radial field non-dynamical while there is a continuous set of minima with the ground state given by $\Psi=v_{0, s s b} e^{i \theta_{a}}$, corresponding to all the possible phases in the circle (if the reader wants to refresh the memory on SSB, see Sect. 3.3.1 and the box that refers to SSB in that section). The pseudo-Goldstone boson $\phi$ is invariant under shift-symmetry, inherited from the $U(1)$ symmetry of the complex scalar field.

However, non-perturbative effects, coming from string theory models or from instantons in the case of the axion, can induce a potential that break the shift symmetry explicitly, although softly, which leads to a residual discrete symmetry. This potential gives a small mass to the ALPs, and has the form

$$
V(\phi)=\Lambda_{a}^{4}\left[1-\cos \left(\frac{\phi}{f_{a}}\right)\right],
$$

where $\Lambda_{a}^{4}$ is the scale of spontaneous symmetry breaking. ${ }^{29}$ This potential is not unique and the overall constant added was chosen arbitrarily. For small field values $\phi \ll f_{a}$,

\footnotetext{
${ }^{29}$ A similar mechanism that generates a dynamical DE component at late times in the context of the DM superfluid model can be seen in Ferreira et al. (2019), presented also briefly in Sect. 4.4.2 of this review.
} 
this potential can be expanded into

$$
V(\phi)=\frac{1}{2} m^{2} \phi^{2}+\frac{g}{4 !} \phi^{4}+\cdots,
$$

where $m=\Lambda_{a}^{2} / f_{a}$ and $g=-\Lambda_{a}^{4} / f_{a}^{4}<0$. Since the spontaneous symmetry breaking scale $f_{a}$ is usually much higher than the explicit symmetry breaking scale $\Lambda_{a}$, the mass is usually very small, with the self-interaction coupling $g$ even smaller. For the QCD case, $\Lambda_{\mathrm{QCD}} \sim 200 \mathrm{MeV}$ (Marsh $2016 \mathrm{a}$ ), and $10^{9} \mathrm{GeV} \lesssim f_{a} \lesssim 10^{17} \mathrm{GeV}$ coming from astrophysical constraints, then we can see that $10^{-10} \mathrm{eV} \lesssim m_{\mathrm{QCD}} \lesssim 10^{-2} \mathrm{eV}$. For string theory models, there is a variety of cases, but typically $\Lambda_{s t} \sim T_{\mathrm{SUSY}} e^{-S_{i}}$, where $S_{i}$ is the instanton action which generally is $S_{i} \gg 1$.

Although very small, given the approximate shift-symmetry, the ALP mass is protected against radiative corrections, and interactions with the standard model are suppressed by powers of $f_{a}$.

For the case of the QCD axion, the mass generated by the non-perturbative QCD effects has a time dependence that scales with the power law of the temperature. For a discussion about that, see, (Marsh 2016a).

Given all that, the action for the ALP is given exactly by the action (93) (usually the self-interaction is omitted since this is suppressed by powers of $f_{a}^{3}$ ).

\subsubsection{Cosmological evolution}

Having established that there is a microscopic theory where the action (93) can come from, we are going to study the cosmology in the general case, which describes any model in the FDM and SIFDM classes. If we ignore the small interaction, this model corresponds to the FDM class. In the presence of interaction, this model corresponds the SIFDM class, where the interaction can be attractive or repulsive, while in the case of ALPs and axions, it can only be attractive $(g<0)$.

We are going to focus only in the matter sector now and omit the Einstein-Hilbert action (which gives GR, the background theory where our field evolves). The action we are going to work with is

$$
S_{\phi}=\int \mathrm{d}^{4} x \sqrt{-g}\left[\frac{1}{2} g^{\mu \nu} \partial_{\mu} \phi \partial_{\nu} \phi-V(\phi)\right],
$$

where the potential can be given by only the mass term, describing the FDM, or both the mass and the interaction, describing SIFDM.

We can study the evolution of this field in a flat Friedmann-Robertson-Walker background (FRW) background, given by the metric $\mathrm{d} s^{2}=\mathrm{d} t^{2}-a^{2}(t) \mathrm{d} x^{2}$, where $a(t)$ is the scale factor. The equation of motion for the ALP is given by

$$
\ddot{\phi}+3 H \dot{\phi}+\frac{\mathrm{d} V}{\mathrm{~d} \phi}=0,
$$

where $H=\dot{a} / a$ is the Hubble parameter. The cosmological evolution is going to depend on the competition between the potential term and the Hubble friction term. 
Let uthe ULDM is subdominant s consider the case of the FDM, where $V(\phi)=$ $(1 / 2) m^{2} \phi^{2}$ to illustrate the cosmological evolution. The background evolution of this field proceeds in the following way. In the early universe, $H \gg m$, the Hubble friction dominates and the solution is a constant given by the initial conditions $\phi_{\text {early }}=\phi\left(t_{i}\right)$. In the case of the ALP, this initial condition is known and given by the formation of the ALP with $\phi_{i}=f_{a} \theta_{a}\left(t_{i}\right)=f_{a} \theta_{i}$. At early times, the ULDM is subdominant and has equation of state $w=-1$ behaving like dark energy. As the universe expands, the Hubble parameter becomes smaller and smaller, until a point where it is smaller than the mass of the field $H \ll m$. The solution of the equation in this case is oscillatory. As the field oscillates, the equation of state also oscillates around zero, giving an averaged out equation of state of a dust like component $w=0$. In this limit, the field behaves like DM and the energy density evolves as $\rho=\rho\left(a\left(t_{*}\right)\right)\left(a\left(t_{*}\right) / a\right)^{3}$, where $t_{*}$ is the time when $H\left(t_{*}\right) \sim m$.

We can already see that the lower the mass is, more and more will take until the Hubble parameter to become smaller than the mass, prolonging the early period where the field behaves as dark energy. For higher masses, the FDM behaves like DM earlier in the history of the universe. Therefore, the mass completely controls when the period of DM domination starts.

In the case of ALP, for them to behave like all the DM of the universe, it has to start oscillating before matter-radiation equality, which gives $m>10^{-28} \mathrm{eV} \sim H\left(a_{\mathrm{eq}}\right)$. However, not all of this regime the ALP as DM presents interesting phenomenological consequences for structures since for heavier masses, ALP behaves closer and closer to CDM. We are going to see this in more detail later, but the sweet spot in mass for the ALP to have this distinct regime on small-scales is $m \sim 10^{-22}-10^{-20} \mathrm{eV}$.

To obtain the full solution of Eq. (98), one needs to solve this equation coupled to the Friedman equation that describes the evolution of the scalar factor according to the components of the universe: $H^{2}=\left(1 / M_{p l}\right)\left(\rho_{\phi}+\rho_{r}+\rho_{b}+\rho_{\Lambda}\right)$, where the contributions in the energy density come from the FDM or SIFDM, radiation, baryons and cosmological constant, respectively. The energy density and pressure of this scalar particle are given by

$$
\rho_{\phi}=\frac{1}{2} \dot{\phi}^{2}+V(\phi), \quad p_{\phi}=\frac{1}{2} \dot{\phi}^{2}-V(\phi),
$$

where in the case that the field is oscillating can be averaged over time. In the case of the FDM, we can take $a(t) \propto t^{p}$, which is valid for the period of radiation and matter dominations, and we have:

$$
\phi(t)=a^{-3 / 2}\left(\frac{t}{t_{i}}\right)^{1 / 2}\left[C_{1} J_{n}(m t)+C_{2} Y_{n}(m t)\right]
$$

where $C_{1,2}$ are determined by the initial conditions, $n=(3 p-2) / 2$ and $J_{n}$ and $Y_{n}$ are the Bessel functions of first and second kind.

We can then compute the relic density of FDM, which is the energy density of those particles today. For the case where FDM is the ALP and behaves like DM, and all the 
DM is made of ALPs, the density fraction is

$$
\Omega_{\mathrm{ALP}} \sim \frac{1}{6}\left(9 \Omega_{r}\right)^{3 / 4}\left(\frac{m}{H 0}\right)^{1 / 2}\left(\frac{\phi_{i}}{m_{p l}}\right)^{2} .
$$

The initial value of the field displacement determines the relic density of ALPs, and to have the DM density observed today, the initial value of the field must be $\phi_{i}>$ $10^{14} \mathrm{GeV}$ for the masses of the ALP that correspond to DM behaviour. A similar calculation can be made for the axion and this can be found in Marsh (2016a).

With that we saw that we have two scales that are important for the DM ALPs: $f_{a}$ the spontaneous symmetry breaking scale that determines the initial conditions of the ALPs, and $\Lambda_{a}$ the scale of explicit breaking that determines the mass given the previous scale. The temperature $T_{*}$ associated with $t_{*}$, the time when the field starts to oscillate and behave like DM, is set after the mass is determined. This is similar in the case of the FDM, where the only one degree of freedom is $m$, although the initial condition $\phi_{i}$ can be unknown. If one assumes the FDM is described by an ALP, then the initial condition is determined.

In the case of the SIFDM, we have an extra parameter in comparison to the FDM, the interaction strength. The interaction term acts as a pressure term in the equation of motion (98). This pressure can be attractive or repulsive. There is then a competition between the Hubble friction, the mass and the pressure. We are going to study more about these effects in the next section.

Cosmological perturbations: We have studied the background evolution and now we need to study the cosmological perturbations. This is important in order for us to study the cosmological consequences of this scenario that can be tested with cosmological observations. We are not going to present here an extensive description, but a summary of the most important results. Notice that like for the cosmological evolution, the procedure here is general for any FDM or SIFDM.

First, we perturb the scalar field and the metric into small perturbations on top of the background values:

$$
g_{\mu \nu}(\mathbf{x}, t)=g_{\mu \nu}^{(0)}(t)+\delta g_{\mu \nu}(\mathbf{x}, t), \quad \phi(\mathbf{x}, t)=\phi_{0}(t)+\delta \phi(\mathbf{x}, t),
$$

where the 0 indicates the background quantities. We are only going to be interested in the scalar perturbations, in this review. We are going to work on conformal time $\eta$ defined as $\mathrm{d} \eta=\mathrm{d} t / a$. The perturbed metric for the scalar metric perturbations is described by four functions (following the convention from Mukhanov 2005)

$$
\mathrm{d} s^{2}=a^{2}(\eta)\left\{(1+2 \Phi) \mathrm{d} \eta^{2}+2 B_{, i} \mathrm{~d} \eta \mathrm{d} x^{i}+\left[(1-2 \Phi) \delta_{i j}-2 E_{, i j}\right] \mathrm{d} x^{i} \mathrm{~d} x^{j}\right\} .
$$

When doing perturbation theory in general relativity, due to gauge invariance, new degrees of freedom that are fictitious and not physical might be introduced. One of the procedures to deal with this is to fix a gauge, which fixes these spurious variables. There are many ways of doing that which leads to the many possible gauges. The final 
physics given by all these gauges is the same, but each gauge offers a better description of different phenomena. The Newtonian gauge $(E=0$ and $B=0)$ is useful in the Newtonian limit. One can also choose the comoving gauge $(B=0$ and $v=0)$, where $v$ is the velocity of the matter fluid. We are going to use both gauges whenever they are useful.

After a gauge is chosen, one substitutes the perturbations in the action (97), ignoring the interaction for simplicity, to obtain the second-order action for the perturbations. And from that one can obtain the equation of motion for the axion perturbation.

We can also re-write the perturbations in terms of the fluid variables: $\rho_{\phi}=\rho_{\phi, 0}+\delta \rho$ and $p_{\phi}=p_{\phi, 0}+\delta p$. From Eq. (99), we can identify the perturbations in the fluid variables with the ones of the scalar field and metric (Hwang and Noh 2009)

$$
\begin{aligned}
\delta \rho= & \left\langle\dot{\phi}_{0} \dot{\delta \phi}-\dot{\phi}_{0}^{2} \Phi+m^{2} \phi_{0} \delta \phi\right\rangle, \quad \delta p=\left\langle\dot{\phi}_{0} \dot{\delta \phi}-\dot{\phi}_{0}^{2} \Phi-m^{2} \phi_{0} \delta \phi\right\rangle, \\
& a(\rho+p) v=k\left\langle\dot{\phi}_{0} \dot{\delta} \dot{\phi}\right\rangle,
\end{aligned}
$$

where we have taken the Fourier transform of the fields with $k$ denotes the wavenumber, and the background pressure and energy density are averaged.

We are interested in obtaining the sound speed of the FDM particle. We showed before that the FDM behaves like dark matter at the background level, but we also need to show that the sound speed is small as expected for dust. This different sound speed is going to give a different Jeans scale and give a different structure formation for this model. Since we are interested in calculating the sound speed, we are going to work in the comoving gauge. In that gauge, the equations, assuming the averaged background equation of state (which is zero), for the scalar perturbations of the metric and fluid simplify and can be combined to give

$$
\ddot{\delta}+2 H \dot{\delta}-4 \pi G \rho \delta+\frac{k^{2}}{a^{2}} \frac{\delta p}{\rho}=\ddot{\delta}+2 H \dot{\delta}+\left(\frac{k^{2}}{a^{2}} c_{s}^{2}-4 \pi G \rho\right) \delta=0,
$$

where $\delta=\delta \rho / \rho$ is the density contrast and $\omega_{k}^{2}=\frac{k^{2}}{a^{2}} c_{s}^{2}-4 \pi G \rho$ is the dispersion relation.

By definition, the sound speed is defined as the term that accompanies the gradient, the term with $k^{2}$. To obtain the expression for the sound speed, we need to compute the perturbation of the pressure $\delta p$. For that, make the simple procedure from Hwang and Noh (2009), where we assume an ansatz for the field perturbation, $\delta \phi(x, t)=$ $\delta \phi_{+}(x, t) \sin (m t)+\delta \phi_{-}(x, t) \cos (m t)$ and substitute that in Eq. (104). This gives us $\delta p$, and the sound speed can be written as:

$$
c_{s}^{2}=\frac{k^{2}}{4 m^{2} a^{2}}\left(\frac{1}{1+\frac{k^{2}}{4 m^{2} a^{2}}}\right) \text {. }
$$

This is the (relativistic) sound speed of our FDM fluid. It is valid outside the Hubble horizon and inside. For sub-Hubble horizon modes, when $k /(\mathrm{ma}) \ll 1$ the sound speed becomes 


$$
c_{s}^{2} \underset{k / m a \ll 1}{\longrightarrow} c_{s, n}^{2}=\frac{k^{2}}{4 m^{2} a^{2}} .
$$

This corresponds to sound speed that can be obtained in the non-relativistic Newtonian theory, as we will see in the next section.

There are two competing terms in Eq. (105), which are the terms inside the dispersion relation. The scale $k_{J}$ for which $\omega_{k}\left(k_{J}\right)=0$ separates the regimes where each of those terms dominates in the equation. We can also write this in terms of $\lambda_{J}=2 \pi a / k_{J}$, the Jeans length. For modes with $\lambda<\lambda_{J}$, the dispersion relation is negative, and the solution of (105) is that the perturbations oscillate. While when $\lambda>\lambda_{J}$, perturbations grow. So there is only gravitational instability for the modes that are outside the Jeans length. In a theory of DM with a finite Jeans length, the growth of perturbations will be suppressed for scales smaller than $\lambda_{\mathrm{J}}$. That is exactly the effect the small mass of the ULDM models has. This leads to important cosmological consequences for these models.

\subsubsection{Evolution on small-scales}

We have finally reached the section where we are going to describe the behaviour of the FDM and of the SIFDM on small-scales. It is this different behaviour that is used to classify the models into different classes.

The action that describes the SIFDM and the FDM is (93). We are interested in studying the behaviour of DM in galaxies, so we are on sub-Hubble scales. In this limit, $H \ll m$ and the field is oscillating fast and behaving as DM. Inside the Hubble horizon and for the small velocities, we have in galaxies $\left(v_{\text {vir }} \ll c\right)$, we are in the non-relativistic limit of our theory. In the Newtonian gauge, the limit where $B=0$ and $E=0$ in (103), and with no anisotropic stress $\Phi=\Psi$, we can write the action for the ULDM field (Niemeyer 2019):

$$
S_{\phi}=\int \mathrm{d}^{4} x a^{3}\left[\frac{1}{2}(1-4 \Phi) \dot{\phi}^{2}-\frac{1}{a^{2}}\left(\partial_{i} \phi\right)^{2}-(1-2 \Phi) V(\phi)\right] .
$$

Since in the non-relativistic limit the field varies slowly, the fast oscillations that we had for the field can be factored and we can re-write the field as

$$
\phi=\frac{1}{\sqrt{2 m a^{3}}}\left(\psi e^{-i m t}+\psi^{*} e^{i m t}\right)
$$

With the field in this form and assuming the $\dot{\psi} \ll m \psi$, we have the total nonrelativistic action, including the Einstein-Hilbert action, that describes this theory given by (Chavanis 2011, 2018)

$$
\begin{aligned}
S= & \int \mathrm{d}^{4} x\left[\frac{i}{2}\left(\psi \partial_{t} \psi^{*}-\psi^{*} \partial_{t} \psi\right)-\frac{|\nabla \psi|^{2}}{2 m}-\frac{g}{16 m^{2}}|\psi|^{4}-m\left(\psi \psi^{*}-\left\langle\psi \psi^{*}\right\rangle\right) \Phi\right. \\
& \left.-\frac{a}{8 \pi G}\left(\partial_{i} \Phi\right)^{2}\right] .
\end{aligned}
$$


The equations of motion of the action yield the Schödinger-Poisson system of equations:

$$
\begin{aligned}
& i \dot{\psi}=-\frac{3}{2} i H \psi-\frac{1}{2 m a^{2}} \nabla^{2} \psi+\frac{g}{8 m^{2}}|\psi|^{2} \psi+m \Phi \psi, \\
& \nabla^{2} \Phi=4 \pi G(\rho-\bar{\rho}) .
\end{aligned}
$$

If we consider time scales much smaller than the expansion, we can ignore expansion of the universe and write the equation of our system as

$$
i \dot{\psi}=-\frac{1}{2 m} \nabla^{2} \psi+\frac{g}{8 m^{2}}|\psi|^{2} \psi+m \Phi \psi
$$

This is the non-linear Schrödinger equation. The gravitational potential term can be re-written in the form $-G m^{2} \psi \int \mathrm{d}^{3} x^{\prime}\left|\psi\left(\mathbf{x}^{\prime}\right)\right|^{2} /\left|\mathbf{x}-\mathbf{x}^{\prime}\right|$. This non-linear equation is the Gross-Pitaesvkii equation described in the previous section and describes the evolution of a wavefunction or a field. We can use this equation to analyze the properties of this system, analytically and numerically.

\section{Fuzzy DM vs SIFDM}

$$
\left\{\begin{array} { l } 
{ i \dot { \psi } = - \frac { 1 } { 2 m } \nabla ^ { 2 } \psi + m \Phi \psi + \frac { g } { 8 m ^ { 2 } } | \psi | ^ { 2 } \psi + \frac { g _ { 3 } } { 1 2 m ^ { 3 } } | \psi | ^ { 4 } \psi + \cdots } \\
{ \nabla ^ { 2 } \Phi = 4 \pi G ( \rho - \overline { \rho } ) }
\end{array} \Longrightarrow \left\{\begin{array}{ll}
g_{i}=0 & \text { Fuzzy DM } \\
g_{i} \neq 0 & \text { SIFDM }
\end{array}\right.\right.
$$

We can also rewrite the field theory above as a set of hydrodynamical-like equations, in this long wavelength limit. For that, if we identify (using the theory in the presence of expansion):

$$
\psi \equiv \sqrt{\frac{\rho}{m}} e^{i \theta}, \quad \mathbf{v} \equiv \frac{1}{a m} \nabla \theta=\frac{1}{2 i m a}\left(\frac{1}{\psi} \nabla \psi-\frac{1}{\psi^{*}} \nabla \psi^{*}\right) .
$$

The vorticity of the superfluid is zero and the momentum density has non-zero curl. The comoving equations of motion for $\psi$ are

$$
\begin{aligned}
& \dot{\rho}+3 H \rho+\frac{1}{a} \nabla \cdot(\rho \mathbf{v})=0 \\
& \dot{\mathbf{v}}+H \mathbf{v}+\frac{1}{a}(\mathbf{v} \cdot \nabla) \mathbf{v}=-\frac{1}{a} \nabla \Phi+\frac{\nabla P_{\text {int }}}{\rho}+\frac{1}{2 a^{3} m^{2}} \nabla\left(\frac{\nabla^{2} \sqrt{\rho}}{\sqrt{\rho}}\right) .
\end{aligned}
$$

These set of equations are the Madelung equations, generalized for an expanding universe. The second term in the right-hand side of equation (116) comes from the self-interaction term, where $P_{\text {int }}$ is the pressure from the interactions. The last term of the second equation is the quantum pressure. This is present even in the absence 
of interaction and it is going to be important for the effects and formation of the condensate for the FDM model. The quantum pressure has the role of not allowing the FDM to cluster and collapse, which also makes the density of this collapsed region to have a finite value. In this way, this model has naturally a cored profile inside the condensate region, addressing the cusp-core problem. This form of the equations is useful for numerical simulations that can reveal some properties of the DM scalar field. However, as we can see from the quantum pressure term, these equations are not defined for $\rho=0$.

The quantum pressure term is present in the ULDM models and it is not present in other candidates of DM. As we discussed above, the behaviour of FDM or SIFDM in each regime depends on a competition between the gravity, the pressure term and the quantum pressure. We can roughly say that then the non-CDM behaviour expected for our models will take place on scales where the quantum pressure term dominates. The sign and size of the interaction might affect this a lot. A naive estimate of this effect is that the scales where this quantum pressure term matters is for scales smaller than the de Broglie wavelength of the particle, $\lambda<\lambda_{\mathrm{dB}}$. The de Broglie wavelength for a typical MW-like galaxy is given by

$$
\lambda_{\mathrm{dB}} \simeq 0.2\left(\frac{m}{10^{-22} \mathrm{eV}}\right)^{-1}\left(\frac{V_{200}}{v}\right) \mathrm{kpc},
$$

where we used the virial velocity (91). For a particle with $m=10^{-22} \mathrm{eV}$, this would mean that the wave-like behaviour of the ULDM particles would be relevant in a MW-like galaxy on scales smaller than $0.2 \mathrm{kpc}$. If we consider dwarf galaxies, for example, where virial velocities are much smaller $v_{\mathrm{vir}}^{\mathrm{dw}} \sim 10 \mathrm{~km} / \mathrm{s}$, these non-CDM effects would take place on scales of order of their halo size. For scales $\lambda>\lambda_{\mathrm{dB}}$, the quantum pressure term is not important anymore and the particles behave like free particles, in a CDM-like way. ${ }^{30}$

This scale where the condensate behaviour becomes important is called the coherent length. We are going to show now a more precise determination of this coherent length in both the FDM and SIFDM. And together with this analysis, it is going to be possible to better understand what is going on in the center of halos that drives this non-CDM behaviour.

\subsubsection{Description of the condensate}

Now that we have our description of the FDM and SIFDM on small scales, we want to understand what takes place inside the halos, where the Schödinger-Poisson equations describe the evolution of the system. We want to describe here what is the picture we have in mind for what happens on those small-scales.

The special feature of the ULDM models is that they present a non-CDM behaviour in galaxies. As we saw in the previous section, the ULDM have a cosmological evo-

\footnotetext{
${ }^{30}$ Here, when I say that is has a CDM-like behaviour, I mean it behaves like a free particle and not like a condensate. Therefore, it follows the hydrodynamical description of CDM. They can have the same type of behaviour like CDM and be described by the same equations, but ULDM have different initial conditions and, for small mass describing DE after equality, can modify the expansion.
} 
lution very close to CDM for large scales. Different than in CDM, the ULDM have a non-zero Jeans length showing that on small-scales this component is going to behave differently than CDM. Therefore, outside galaxies, ULDM behaves like CDM but with a suppressed power spectrum, and inside galaxies, in those homogeneous sub-Jeans scales ULDM can have a non-CDM behaviour.

Inside these homogenous sub-Jeans regions inside galaxies, the ULDM thermalizes and forms gravitationally bound compact objects, called Bose stars or solitons, where a Bose-Einstein condensation or superfluid is formed. This was described in many references in the literature (Lee and Pang 1992; Jetzer 1992; Kolb and Tkachev 1993; Guth et al. 2015; Semikoz and Tkachev 1997; Khlebnikov 2000; Sikivie and Yang 2009; Erken et al. 2012), both in the presence and in the absence of interactions. The coherence length of this condensate sets the region where the wave behaviour of the condensate is important and changes the dynamics. Outside the condensate, on scales larger than the coherent length, the ULDM behaves as particles following the particle description as CDM (decoupled axion following (Sikivie and Yang 2009)), with different initial conditions than in CDM.

There have been many studies of the properties of these condensed gravitationally bounded objects, in particular in the context of axions (Ruffini and Bonazzola 1969a; Chavanis 2011; Barranco and Bernal 2011; Eby et al. 2015, 2016a, b, 2019, 2018; Braaten et al. 2016; Visinelli et al. 2018). In this section, we are going to study the thermalization and formation of these compact objects not only in the context of FDM, but also in the context of the SIFDM. It is interesting to see that the size and phenomena, BEC or superfluidity, described by each of these models can differ a lot in each case, and in the case of the SIFDM it differs with the sign of the self-interaction.

The formation process of this Bose-Einstein condensate by gravitational interaction in the center of halos or in axion miniclusters is shown to take place in our universe, with relaxation times dominated by faster gravitational relaxation time, which is smaller than the age of the universe (Semikoz and Tkachev 1997; Khlebnikov 2000; Sikivie and Yang 2009; Schive et al. 2014a; Levkov et al. 2018; Kirkpatrick et al. 2020). We are going to show here we can describe this formation of the condensate and obtain the relaxation times in the case of the FDM and the SIFDM.

Summarizing, the picture that we have to have in mind is shown in Fig. 10. Inside the halos of galaxies, a condensate core is formed. ${ }^{31}$ These Bose clumps are called solitons or Bose stars. This is also the picture that we have for the DM superfluid model, presented in Sect. 4.2.

As we saw from the description of the ULDM given above, we are describing the theory purely classically. It is valid then to ask if our ULDM can be treated as a classical theory or not, especially since this model has such small masses.

Classicality is an emergent concept that can be applied for system that are composed by a large number of constituents. In this limit, the quantum effects of the theory are suppressed by this macroscopic number of particles, and the theory can be described by a classical theory. In this way, there is a limit where this classical approximation breaks and the quantum effects become important.

\footnotetext{
31 The picture described here can differ in the entire literature. We are going to discuss this in Sect. 4.1.5.
} 


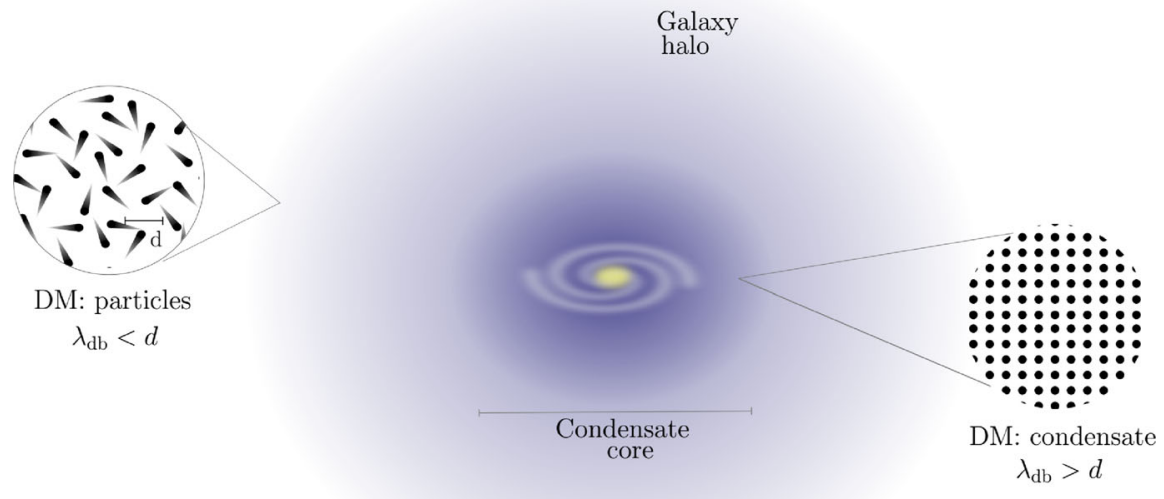

Fig. 10 Schematic figure of the behaviour of ULDM in galaxies, where a condensate core is expected to form in the inner parts of the galaxy, while DM behaves like normal DM in the outskirts or outside galaxies

Lets first talk about what happens in the halo. When taking the classical limit of a theory, we can think that we decompose our quantum field $\hat{\psi}$ into

$$
\hat{\psi}=\psi+\delta \hat{\psi}
$$

where $\langle\hat{\psi}\rangle=\psi$ is the classical field (since if it was quantum this expectation value would be zero) and $\delta \hat{\psi}$ are the quantum corrections on top of the classical field. These quantum correction are suppressed by large occupancy number of the states, and for a coherent state is given by: $\delta \hat{\psi} / \psi \propto 1 / \mathcal{N}$. Therefore, if we are in a system with a large number of quantum constituents, the quantum corrections can be neglected and the system can be treated as classical. And this classical field is going to obey classical equations. This is, very roughly since this can be made much more precise mathematically, the definition of a classical field. On top of that, it is a condensate, there is a large occupation number of the ground state, so $\mathcal{N}=\mathcal{N}_{0}$.

We can then estimate the occupancy number that we have in a halo. If we consider a MW-like galaxy, and we take as an example the axion, then the number density of axions in the galaxy is

$$
n_{\mathrm{gal}}=\frac{\rho_{\mathrm{gal}}}{m} \approx \frac{\mathrm{GeV} / \mathrm{cm}^{3}}{10^{-5} \mathrm{eV}} .
$$

Since we can write the occupation number roughly as $\mathcal{N} \sim n \lambda_{\mathrm{dB}}^{3}$, given the de Broglie wavelength of an axion with mass $m \sim 10^{-5} \mathrm{eV}$, we have that in a galaxy $\mathcal{N} \sim 10^{46}$. This shows that in galaxies today, this ultra-light particle has a huge occupancy number and we can consider a classical evolution. We can extend this estimation for earlier 
times, if we assume the QCD axion, where the potential arises from non-perturbative effects on scales $\Lambda_{\mathrm{QCD}}$. For those axions, the energy density (assuming they are most or all the DM in the universe) is given by $n_{a} \sim \rho_{a} / m \sim\left(T_{\text {eq }} / T_{\mathrm{QCD}}\right) \rho_{\mathrm{tot}} \sim T_{\mathrm{eq}} T_{\mathrm{QCD}}^{3} / m$. The de Broglie wavelength can be estimated to be at most of the size of the Hubble horizon at that time $\lambda \sim H_{\mathrm{QCD}}^{-1} \sim T_{\mathrm{QCD}}^{2} / M_{p l}$. This yields an even larger occupation number $\mathcal{N} \sim 10^{61}$.

If you start your theory with a classical scalar field described by classical equations of motion, then this description is valid for almost all the evolution of the universe (early times might require quantum treatment tough), specially the cosmological times we are considering. So it is a good description for the evolution of the ULDM ${ }^{32}$.

As we saw in Sect. 3, in the classical limit, we can describe the properties of the (classical) condensate using a classical theory. And that is what we are going to do here. In the classical point of view, the condensate formed is going to be described by a slowly varying in space, homogeneous and stable field that presents long-range correlation (Guth et al. 2015). In the case of FDM and SIFDM, condensation takes place in the presence of gravity, and of both gravity and self-interactions in the case of SIFDM. So we need to show how this gravitational thermalization takes place. We are going to describe these condensates, showing their size, given by their correlations length, and the condensate solution using linear theory.

\section{SIFDM}

We are first going to treat the SIFDM model, which is described by the presence of a self-interaction (Chavanis 2011; Guth et al. 2015; Chavanis 2016, 2018). We specialize here to the two-body interaction, since higher order interaction are usually suppressed in low-energy systems like the one we are interested. But it is easy to generalize this for higher order interactions. From what we saw in the previous section, the theory of a self-interacting condensate describes a superfluid in certain regimes. We are going to see here for which conditions this occurs in the SIFDM model.

We are going to work in the limit where we ignore gravity to investigate the effect of the self interaction in the model in a very similar way as described in Sect. 3.3. The Schrödinger equation that describes model is given by

$$
i \dot{\psi}=-\frac{1}{2 m} \nabla^{2} \psi+\frac{g}{8 m^{2}}|\psi|^{2} \psi
$$

We decompose the field into a homogeneous background solution, which represents the condensate, plus a perturbation part: $\psi(\mathbf{x}, t)=\psi_{\mathrm{c}}(t)+\delta \bar{\Psi}(\mathbf{x}, t)$. The condensate part satisfies the Gross-Pitaevskii equation,

$$
i \dot{\psi}_{\mathrm{c}}=\frac{g}{8 m^{2}}\left|\psi_{0}\right|^{2} \psi_{\mathrm{c}}
$$

that has a simple periodic solution $\psi_{\mathrm{c}}(t)=\psi_{0} e^{-i \mu_{\mathrm{c}} t}$, where $\left|\psi_{0}\right|^{2}=n_{0}$ is the number density of particles that fixes the amplitude of $\psi_{0}$, and $\mu_{\mathrm{c}}=g n_{0} / 8 \mathrm{~m}^{2}$.

\footnotetext{
32 There is one subtly here that there might be effects on local systems that can deplete the condensate and break coherence. I will discuss that in Sect. 4.1.5.
} 
The equation describing the evolution of the perturbation, making the field redefinition $\delta \bar{\Psi}=\psi_{\mathrm{c}} \delta \Psi$, is

$$
i \delta \Psi=-\frac{1}{2 m} \nabla^{2} \delta \Psi+\frac{g n_{0}}{8 m^{2}}\left(\delta \Psi+\delta \Psi^{*}\right) .
$$

Since $\delta \Psi$ is a complex scalar field, we can decompose the field into a real and a imaginary parts, $\Psi=A+i B$. We want to determine the dispersion relation of this system, so we write the equation of motion in Fourier space:

$$
\frac{\mathrm{d}}{\mathrm{d} t}\left(\begin{array}{l}
A_{k} \\
B_{k}
\end{array}\right)=\left(\begin{array}{cc}
0 & \frac{k^{2}}{2 m} \\
-\frac{k^{2}}{2 m}-\frac{g n_{0}}{4 m^{2}} & 0
\end{array}\right)\left(\begin{array}{l}
A_{k} \\
B_{k}
\end{array}\right),
$$

where we call $\zeta_{k}=k^{2} / 2 m+g n_{0} / 4 m^{2}$. The dispersion relation is given by

$$
\omega_{k}^{2}=\frac{g n_{0}}{4 m^{2}} \frac{k^{2}}{2 m}+\frac{k^{4}}{4 m^{2}} .
$$

We can see that for $\omega_{k}^{2}>0$ we have an oscillatory solution:

$$
\delta \Psi_{k}=Z\left(\omega_{k}+\zeta_{k}\right) e^{i \omega_{k} t}+Z^{*}\left(\omega_{k}-\zeta_{k}\right) e^{-i \omega_{k} t}
$$

where $Z$ is an arbitrary complex parameter. When $\omega_{k}^{2}<0$, the solution of the equation for $\delta \Psi_{k}$ is given by exponentials,

$$
\delta \Psi_{k}=c_{1}\left(\gamma_{k}-i \zeta_{k}\right) e^{\gamma_{k} t}+c_{2}\left(\gamma_{k}+i \zeta_{k}\right) e^{-\gamma_{k} t}
$$

where $\gamma_{k}=(k / \sqrt{2 m}) \sqrt{-\zeta_{k}}$ are the eigenvalues of the matrix, and $c_{1}$ and $c_{2}$ are constants given by the initial conditions.

The regimes where the dispersion relation is positive or negative are separated by the modes with wavenumber,

$$
\omega_{k}^{2}=0 \Longrightarrow k_{*}^{2}=-\frac{g n_{0}}{2 m}
$$

from where we can also determine the wavelength $\lambda_{*}=2 \pi / k_{*}$ that divides these regimes. This wavelength is proportional to the healing length calculated in the previous sections. However, can see from this scale that what is actually going to determine if we have a stable oscillatory solution or a exponentially growing instability is the sign of the interaction.

For a repulsive interaction, $g>0$, the homogeneous configuration is always stable, and it is always going to be described by an oscillatory solution, either if $\lambda$ is bigger or smaller than $\lambda_{*}$. This means that for all wavelength we can have a stable solution that can describe a condensate. From the dispersion relation, we can also see that, in the long wavelength regime

$$
\omega_{k} \simeq c_{s} k
$$


which is the dispersion relation of the phonon, that propagates as a wave, mediating long-range correlation. This means that the SIFDM with a repulsive interaction is a superfluid, with the theory fully described by this propagating phonon. On the other hand, for very small wavelengths, large $k$, the term $\omega_{k}=k^{2} / 2 m$ dominates, which is the dispersion relation of a free massive particle, and the system stops exhibiting superfluidity. In the intermediary regime, where we should consider both terms of the dispersion relation, and the theory described by two degrees of freedom the phonon and the massive particle associated with particle creation away from the condensate. The scales that determine what we mean by long-wavelength regime is where $\lambda \gg \lambda_{*}$ in a way that the linear term dominates the dispersion relation. Since $\lambda_{*}$ is proportional to the healing length, this condition for the long wavelengths is equivalent to the condition in Sect. 3.3 that the healing length gives us the scale where quantum pressure (QP) can be neglected, which is what we are describing here.

When we have an attractive interaction, given by $g<0$, we have two regimes of stability. For $k<k_{*}\left(\lambda>\lambda_{*}\right)$, we have exponential growing solutions, which means that perturbations grow parametrically. Given this instability, the condensate cannot be formed on these scales. For $k>k_{*}\left(\lambda<\lambda_{*}\right)$, the solution oscillates and is stable, forming a condensate. This stable configuration, however, is different than in the case for repulsive interaction, forming a localized object, with maximum size given by $\lambda_{*}$. This localized stable solutions are called soliton. Therefore, it makes sense that this is the healing scale is the scale of the stability, since this is the scale below which the interaction "heals" perturbations of the condensate.

$$
\begin{aligned}
& \mathrm{g}>0 \\
& \mathrm{~g}<0
\end{aligned} \longrightarrow \begin{cases}\forall \lambda & \text { Solution oscillates. Condensate (long range) } \\
\lambda>\lambda_{*} & \text { Structures grow. No condensate. } \\
\lambda<\lambda_{*} & \text { Solution oscillates. Condensate (finite size) }\end{cases}
$$

The case of the attractive interaction is not a superfluid even in the stable localized regions, the solitons. The only stable regions are for $\lambda<\lambda_{*}$ and this is the regime where the linear term is always subdominant in the dispersion relation. The longwavelength regions in this case are the regions where instability happens and there is no formation of a condensate.

Condensate solution Having studied the stability of the system and determined the regimes where we have stable and unstable solutions, we can now describe the background solution for each case. As we saw in the "condensate solution" part of Sect. 3.3, one possible solution for the weakly interacting BEC is given by the solitons. For repulsive interactions, the condensate solution is a dark soliton, while for a attractive interaction one has a bright soliton.

What is important is that for the repulsive interaction we can create a condensate of any size desired. So the size of the condensate is not limited and it will depend only on the choice of mass and strength of the interaction. For the case of attractive interaction, we can only have localized stable solutions, giving a finite size bright soliton, with maximum size $\lambda_{*}=2 \pi \sqrt{2 m /|g| n_{0}}$. Therefore, we can see that we could only have a soliton, for an attractive interaction, that is relevant on galactic scales if the mass 
is very small but with a not very small coupling. An interplay between these two parameters need to occurs then if one wants galactic sized $\lambda_{*}$.

This condition also shows us that the QCD axion is not a good candidate for a ULDM (at least not to represent most of the ULDM, but it can still represent a fraction of DM). For the QCD axion the interaction, given by $g_{\mathrm{a}}=-\Lambda^{4} / f_{\mathrm{a}}^{4}$ is negative, and it is extremely small with $g_{\mathrm{a}} \sim-10^{-48}$, because $\Lambda \sim 0.1 \mathrm{GeV}$, for the typical QCD scale, and $f_{\mathrm{a}} \sim 10^{11} \mathrm{GeV}$, for typical Peccei-Quinn scale. Given that the mass of the axion is approximately $m \sim 10^{-5} \mathrm{eV}$, the soliton length, for $n_{0} \sim n_{\text {gal }}$, is $\lambda_{\mathrm{s}} \sim 2.8 \times 10^{11} \mathrm{~km} \sim 9 \times 10^{-6} \mathrm{kpc}$. This is much smaller than any galactic scales, which are of the order of tens to hundreds of kpc. The QCD axion, then, produces these small and localized clumps of axions.

Occupancy number evolution - With those solutions in hand, we can understand how the evolution of the occupancy number for the condensate will behave for each mode. Determining the evolution of the occupation number is very important since having high occupancy number of the ground state of the system st what is actually the definition of a condensate. And this definition is independent of the system described, representing the best way of showing that condensation took place. This is given by $\mathcal{N}=\left|\psi_{k}\right|^{2} / V$, where $\psi_{k}$ is constructed from the exponential and oscillatory solutions described above, with a random phase. The average occupation number evolves as

$$
\begin{array}{ll}
\left\langle\mathcal{N}_{k}(t)\right\rangle=\left\langle\mathcal{N}_{k}\left(t_{i}\right)\right\rangle\left\{1+\frac{1}{2 \gamma_{k}^{2}}\left(\frac{g n_{0}}{4 m^{2}}\right) \sinh ^{2}\left[\gamma_{k}\left(t-t_{i}\right)\right]\right\}, & \text { for } \omega_{k}^{2}<0 \\
\left\langle\mathcal{N}_{k}(t)\right\rangle=\left\langle\mathcal{N}_{k}\left(t_{i}\right)\right\rangle\left\{1+\frac{1}{2 \omega_{k}^{2}}\left(\frac{g n_{0}}{4 m^{2}}\right) \sin ^{2}\left[\omega_{k}\left(t-t_{i}\right)\right]\right\}, & \text { for } \omega_{k}^{2}>0
\end{array}
$$

For $g>0$, an repulsive interaction, $\gamma_{k}$ is imaginary, with $\gamma_{k}=i \omega_{k}$, so the occupation number oscillates and the oscillations are stable. The ratio $\left\langle\mathcal{N}_{k}(t)\right\rangle /\left\langle\mathcal{N}_{k}\left(t_{i}\right)\right\rangle$ which has the largest value is obtained for modes that minimize $\omega_{k}$, which are the modes with $k \rightarrow 0$. These are the longest wavelengths. Since the long wavelength dominates, this means that long-range correlation is present, and we can have a long range condensate.

For $g<0$, the occupation number grows exponentially. The fastest growth is given by the modes $k=k_{*}$ that maximize $\gamma_{k}$. So the modes $k>k_{*}$ or $\lambda<\lambda_{*}$, where $*$ denotes the characteristic scale where instability sets in, will dominate and the stable configuration of the system will be localized clumps. The size of these clumps will be given by the mass and interaction of the model.

FDM: only gravity

We are now going to describe a model without interaction, where the ultra-light particles are under the influence of the gravitational potential. We can think that gravitational potential has the same effect as an attractive interaction, in a way that quantum pressure has to counteract the gravitational collapse. This gives a good picture to what to expect, from the knowledge obtained for the SIFDM. However, one needs to remember that the FDM model is described by a non-interacting theory, which means that it condenses into a BEC, but does not exhibit superfluidity in any regime. 
The Schrödinger equation for DM in a gravitational potential, in the absence of interaction is

$$
i \dot{\psi}=-\frac{1}{2 m} \nabla^{2} \psi+m \Phi \psi
$$

which is coupled to the Poisson equation:

$$
\nabla^{2} \Phi=4 \pi G\left(m|\psi|^{2}-\bar{\rho}\right)
$$

where the average background density, $\bar{\rho}$, was subtracted. Expanding the field as done previously $\psi(\mathbf{x}, t)=\psi_{\mathrm{c}}(t)+\delta \Psi(\mathbf{x}, t)$, the equation for the condensate is trivial and $\psi_{\mathrm{c}}=\psi_{0}=$ const.. For the fluctuations, we can write the linearized systems of equations that govern the evolution of the perturbation:

$$
\begin{aligned}
& i \delta \dot{\Psi}=-\frac{1}{2 m} \nabla^{2} \delta \Psi+m \Phi, \\
& \nabla^{2} \Phi=4 \pi G m n_{0}\left(\delta \Psi+\delta \Psi^{*}\right) .
\end{aligned}
$$

These can be combined into the equation:

$$
i \delta \dot{\Psi}=-\frac{1}{2 m} \nabla^{2} \delta \Psi+4 \pi G m^{2} n_{0} \nabla^{-2}\left(\delta \Psi+\delta \Psi^{*}\right) .
$$

One can notice that the equation above is very similar to the equation we had for the interacting case (122) for an attractive interaction. With that, we expect that there is an instability for long wavelengths, and that the condensate stable solution is only given for a finite region, forming a localized core. To determine, this lets take the Fourier transform of the fields. Like in the interacting case, the instability is divided by the regimes where the dispersion relation is smaller or bigger than zero. For the parameters of the FDM, we can determine the wavenumber that separates the regimes as

$$
\tilde{\omega}_{k}^{2}=0, \Longrightarrow k_{\mathrm{J}}=\left(16 \pi G m^{3} n_{0}\right)^{1 / 4}
$$

This scale is the Jeans scale and it separates the regimes where gravity dominates and collapse happens $\left(k<k_{\mathrm{J}}\right)$, and the regime where the quantum pressure dominates and the solution is stable and oscillates $\left(k>k_{\mathrm{J}}\right)$. In this regime, we can have a condensate. The quantum pressure term counteracts the gravitational attraction and any attempt to localize the particle is accompanied by an increase in energy. So stability below the Jeans scale arises because of the uncertainty principle.

$$
\begin{cases}\lambda>\lambda_{\mathrm{J}} & \text { Structures grow. No condensate. } \\ \lambda<\lambda_{\mathrm{J}} & \text { QP dominates, solution oscillates. Condensate (finite size) }\end{cases}
$$


We can estimate the size of the coherent condensate core. Rewriting the Jeans wavelength as

$$
\begin{aligned}
\lambda_{\mathrm{J}} & =\frac{2 \pi}{k_{\mathrm{J}}}=\frac{\pi^{3 / 4}}{2}(G \rho)^{-1 / 4} m^{-1 / 2} \\
& =94.5\left(\frac{m}{10^{-22} \mathrm{eV}}\right)^{-1 / 2}\left(\frac{\rho}{\rho_{\text {crit }}}\right)^{-1 / 4}\left(\frac{\Omega_{\mathrm{m}} h^{2}}{0.12}\right)^{-1 / 4} \mathrm{kpc}
\end{aligned}
$$

where $\rho_{\text {crit }}$ is the critical density. For fuzzy $D M$ in an overdense region $\rho=10^{6} \rho_{\text {crit }}$,

$$
\text { Fuzzy DM: } m \sim 10^{-22} \mathrm{eV} \longrightarrow \lambda_{J}^{F D M} \sim 3 \mathrm{kpc}
$$

forming a condensate that is of the order of the scales of the halo of galaxies. As we can see, for a MW-like galaxy, this core formed is smaller than the halo. So we expect that in the outskirts of the halo, the DM is not going to be condensed and is going to behave like normal matter, with the profile following the NFW profile. For the QCD axion, if we assume that we can have an axion without interaction, $\lambda_{\mathrm{J}} \sim 1.7 \times 10^{-7} \mathrm{kpc}$, which is a very small-scale in comparison to galaxies. This stable bound system is called a Bose star.

Adding the expansion of the universe, we can see that the system of equations becomes

$$
\begin{aligned}
& \frac{i}{a^{3 / 2}} \partial_{t}\left(a^{3 / 2} \psi\right)=-\frac{1}{2 m} \frac{\nabla^{2} \psi}{a^{2}}+m \Phi \psi, \\
& \nabla^{2} \Phi=4 \pi G a^{2}\left(m|\psi|^{2}-\bar{\rho}\right) .
\end{aligned}
$$

Like before, we determine the linearized equations for the perturbations $\delta \Psi$ around the coherent homogeneous background that evolves as $\psi_{\mathrm{c}} \propto a^{-3 / 2}$. The equation that describes the evolution of the perturbation is

$$
i \delta \dot{\Psi}_{k}=-\frac{k^{2}}{2 m a^{2}} \delta \Psi_{k}-\frac{3}{2} m \Omega_{\mathrm{a}} \frac{H^{2} a^{2}}{k^{2}}\left(\delta \Psi_{k}+\delta \Psi_{k}^{*}\right)
$$

where $\Omega_{\mathrm{a}}=m n_{0} / \rho_{\text {tot }}$ is the density parameter of our FDM particles. The real part of the perturbations obeys the following equation:

$$
\ddot{A}_{k}+2 H \dot{A}_{k}-\frac{3}{2} \Omega_{\mathrm{a}} H^{2} A_{k}+\left(\frac{k^{2}}{2 m a^{2}}\right)^{2} A_{k}=0 .
$$

All the terms in this equation would also be present from a massive DM component like CDM, except for the last term, which is related to the quantum pressure. The Jeans length in this case is given by

$$
\frac{k_{\mathrm{J}}}{a}=\left(6 \Omega_{\mathrm{a}}\right)^{1 / 4} \sqrt{H m} .
$$


This stability analysis shows us that if we want to construct a ULDM model with the desired feature of having a condensate in galactic scales, for the FDM model we need to have a very small mass of the order $m \sim 10^{-22} \mathrm{eV}$. This mass is within the bounds we had obtained earlier for all the ULDM models. For the FDM model, the only parameter that controls the size of the condensate is the mass. We need then to determine the mass of the FDM particle for which this model can address the smallscale problems, and for which the model is still in accordance with the very precise cosmological observations. This is what we show in the next subsection. We are going to put bounds on this parameter according to the observations in galaxies and on large-scales.

Condensate solution The ground state solution for the FDM is called Bose star, and it is a gravitational bound stable state in 3-dimensions. This can be obtained by minimizing the Hamiltonian that is described by system with gravity alone, at fixed particle number $(N)$ (Guth et al. 2015). In the absence of an exact solution, one can have an ansatz for this solution, in analogy to the ground state of the hydrogen atom, we assume spherical symmetry and have

$$
\psi_{\mathrm{bs}}(r)=\psi_{s} e^{-i \mu_{s} t}=\sqrt{\frac{N k_{\mathrm{bs}}^{3}}{\pi}} e^{-k_{B S} r} e^{-i \mu_{s} t},
$$

which corresponds to the ground state $E=-25 G^{2} m^{5} N^{3} / 512, \mu_{s}<0$ and where the characteristic wavenumber of the Bose star is given by

$$
k_{\mathrm{bs}}=5 G m^{3} N / 16 \sim k_{\mathrm{J}},
$$

given that $N \sim n_{\mathrm{bs}} / k_{\mathrm{bs}}^{3}$. Therefore, the Bose star wavelength coincides with the Jeans length that determines the core, the stable region with no gravitational collapse.

However, the study of the Bose star solution needs to be done numerically. We are going to see next how the formation of these Bose stars takes place in kinetic theory. We see the formation of the BEC in these gravitationally bound structures, having the Bose star, and see how this Bose star grows. We are also going to discuss the cores formed by other simulations in Sects. 4.1.6 and 4.3. With those we can determine the profile, mass and size of the core.

In the case of the SIFDM, we saw that what determines the size of the condensate is the mass and of the strength of the interactions. Like for the FDM, to construct a SIFDM model that addresses the small-scale problems and that are allowed by current observations, we need to put bounds on those parameters. This model was much less studied than the FDM model. There are a few works that present some bounds on those parameters (see references in the definition of this class), but with much less constraints than in the FDM case. This is also understandable since here we have an extra parameter in the model. A more complete analysis for the bounds of the SIFDM is going to be presented in a future publication and it is being considered by the authors while this review is being written. 


\section{Initial evolution of the condensate}

The picture that we have for the ULDM is that in the interior of galaxies it forms a core where the ULDM condenses and forms a Bose-Einstein condensate, in the case of FDM, and a superfluid, in the case of the SIFDM (and of the DM superfluid which we will see in the next section). Previously, we have determined the region where condensation can happen in the halo and calculated the coherence length of the condensate in the case of FDM and SIFDM, and calculating what is the ground state solution of the condensate, the soliton or Bose star, for each case.

We want to study if there is the formation of a BEC in the center of galaxies in the presence of gravitational interactions, on top of the self-interaction in the case of SIFDM. We showed before that the ULDM is described by a classical theory. The reader, then, might be wondering that since we are using a classical theory we cannot fully describe thermalization and the formation of the condensate. Indeed, as we showed in Sect. 3.6, and also in Guth et al. (2015), classical fields follow a RayleighJeans distribution which present the UV catastrophe, and, thus, cannot describe a condensate. However, Guth et al. (2015) showed that a classical field theory is capable to describe the phase transition that represents the formation of a condensate and the theory has a well defined thermal equilibrium if one introduces a ultra-violet cutoff in the theory. We saw the same conclusion in wave turbulence, in Sect. 3.6, where the four-wave classic kinetic equation can only describe the initial stages of a BEC if a ultra-violet cutoff is present in the theory. Therefore, it is possible to describe the formation of a condensate with a classical theory.

To study the formation of a BEC in the center of galaxies here we use the kinetic equation from wave turbulence. We can use the four wave kinetic equation to describe the initial stages of evolution of a BEC. This formalism can be used in the case of ULDM to show that Bose-Einstein condensation caused by gravitational forces indeed happens in the center of halos (and of axion miniclusters). From this theory, we can also obtain a prediction for the condensation time and obtain the properties of the condensate distribution. This was done in Levkov et al. (2018) that made this analysis for the FDM model, and showed numerically the formation of the condensate and growth of the Bose stars, and in Kirkpatrick et al. (2020) where the role of interaction was also studied. This is what we are going to show now.

We are going to proceed in the same fashion as in Sect. 3.6, but with the system subjected to a gravitational potential. The difference from the previous procedure is that the gravitational interaction is long range, while in Sect. 3.6, we studied only the role of short-range interactions.

In galaxies, we are in the regime of high occupation number, and the classical description is valid. For the system we are considering, $p^{2} \ll 2 m T$; therefore, for the period where the evolution is going to be described by this kinetic theory, the UV catastrophe is not a problem, and the classical kinetic description of the system is valid.

In this regime, we can see that this system described by the non-linear Schrödinger equation (113) can be described in wave turbulence by the classical kinetic equation (83) where the non-linear process is the four-wave resonant interaction. In the case of the ULDM subjected to gravitational potential, the kinetic equation is given by 


$$
\frac{\partial f}{\partial t}+\frac{\mathbf{p}}{m} \cdot \nabla_{x} f=2 \operatorname{Im} \int \mathrm{d} \mathbf{y} e^{-i \mathbf{p . y}}\left\langle\psi\left(\mathbf{x}+\frac{\mathbf{y}}{2}\right) \psi^{*}\left(\mathbf{x}-\frac{\mathbf{y}}{2}\right) U_{\text {tot }}\left(\mathbf{x}+\frac{\mathbf{y}}{2}\right)\right\rangle,
$$

where $f$ is the Wigner distribution described by (82) and the potential $U_{\text {tot }}$ is given by

$$
\begin{aligned}
U_{\mathrm{tot}}(\mathbf{x})= & U_{\mathrm{G}}+g|\psi(\mathbf{x})|^{2}=4 \pi G m \int \mathrm{d} \mathbf{x}^{\prime} \Delta_{\mathbf{x}-\mathbf{x}^{\prime}}^{-1}\left(|\psi(\mathbf{x})|^{2}-n\right) \\
& +g \int \mathrm{d} \mathbf{x}^{\prime} \delta\left(\mathbf{x}^{\prime}-\mathbf{x}\right)|\psi(\mathbf{x})|^{2},
\end{aligned}
$$

and $\Delta_{\mathbf{x}-\mathbf{x}^{\prime}}^{-1}$ is the Green function coming from the Poisson equation

$$
\Delta_{\mathbf{x}-\mathbf{x}^{\prime}}^{-1}=\frac{1}{4 \pi\left|\mathbf{x}-\mathbf{x}^{\prime}\right|} .
$$

These two equations are equivalent in this limit to the Schrödinger-Poisson system that describes the evolution of the ULDM field in galaxies. This is the Landau kinetic equation for the gravitating ensemble of random phase classical waves inside a structure of radius $R$ that in our case of interest is the halo of a galaxy, but it can be an axion minicluster or a periodic box, in the case of simulations.

To obtain the closed form of this kinetic equation we need to make some assumptions, as detailed in Sect. 3.6. In our case, we are going to assume as a initial distribution Gaussian distributed ULDM particles, described by a Gaussian random field $\left|\tilde{\psi}_{\mathbf{p}}\right|^{2}=8 \pi^{3 / 2} \tilde{N} e^{-\tilde{p}^{2}}$ with random phases $\arg \tilde{\psi}_{\mathbf{p}}$, where $\psi=\tilde{\psi} v_{0}^{2} \sqrt{m / G}$, $\tilde{\mathbf{p}} \equiv \mathbf{p} / m v_{0}$ and $v_{0}$ is the initial velocity. This initial configuration is the Fourier transform of $\tilde{\psi}(t, \tilde{\mathbf{x}})=\tilde{\psi}(0, \tilde{\mathbf{x}})$, with $\tilde{\mathbf{x}}=m v_{0} \mathbf{x}$, which is an isotropic and homogeneous field. This is an uncorrelated field, a field that has minimal coherence length. This type of initial configuration reinforces that there is no seed for condensation in the halo or axion minicluster, and that condensation arises simply by the gravitational interaction or gravitational interaction plus self-interaction. This initial condition is also well motivated from axions formed by the Kibble mechanism (Fairbairn et al. 2018).

Using the Wick theorem, we can write our kinetic equation in closed form for the SIFDM as (Kirkpatrick et al. 2020)

$$
\frac{\partial f}{\partial t}+\frac{\mathbf{p}}{m} \cdot \nabla_{x} f=F_{1}+F_{2}+I(f)
$$

where the $F_{1}$ and $F_{2}$ correspond to the two point correlation functions in the Wick theorem, the first two terms in Eq. (84), and $I(f)$ comes from the connected part $^{33}$,

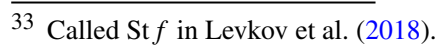


the last term of Eq. (84). The first two terms are given by

$$
\begin{aligned}
F_{1}= & 2 \operatorname{Im}\left\langle U_{\text {tot }}\left(\mathbf{x}-i \nabla_{\mathbf{p}} / 2\right)\right\rangle f(\mathbf{x}, \mathbf{p}) \\
F_{2}= & \frac{2}{(2 \pi)^{6}} \operatorname{Im} \int \mathrm{d} \mathbf{q} \mathrm{d} \mathbf{q}^{\prime} \mathrm{d} \mathbf{y} \mathrm{d} \mathbf{y}^{\prime}\left[4 \pi G m^{2} \Delta_{\mathbf{y}}^{-1}+g \delta(\mathbf{y})\right] e^{i\left(\mathbf{y}^{\prime} \cdot \mathbf{q}-\mathbf{y} \cdot \mathbf{q}^{\prime}\right)} \\
& f\left(\mathbf{x}+\frac{\mathbf{y}}{2}, \mathbf{p}+\mathbf{q}\right) f\left(\mathbf{x}+\frac{\mathbf{y}^{\prime}}{2}, \mathbf{p}+\mathbf{q}^{\prime}\right) .
\end{aligned}
$$

They are $T$-odd terms. They are order one in the interactions $\mathcal{O}(g)$ and $\mathcal{O}(G)$. The potential obeys: $\Delta\left\langle U_{\text {tot }}\right\rangle(\mathbf{x})=(4 \pi G m) /(2 \pi)^{3} \int \mathrm{d} \mathbf{p}(f(\mathbf{x}, \mathbf{p})-n)$.

We can separate the contribution in $I(f)$ by coming from the the self-interaction and from the gravitational interaction $I(f)=I_{g}(f)+I_{\mathrm{G}}(f)$, respectively, where

$$
I_{g}=2 \operatorname{Im} \int \mathrm{d} \mathbf{y} e^{-i \mathbf{p} \cdot \mathbf{y}} g\left\langle\psi_{+} \psi_{-}^{*} \psi_{+} \psi_{+}^{*}\right\rangle_{\mathrm{conn}}, \quad I_{\mathrm{G}}=2 \operatorname{Im} \int \mathrm{d} \mathbf{y} e^{-i \mathbf{p} \cdot \mathbf{y}}\left\langle\psi_{+} \psi_{-}^{*} U_{\mathrm{G}}\right\rangle
$$

Here we simplified the notation by writing $\psi_{ \pm}=\psi(\mathbf{x} \pm \mathbf{y} / 2)$. These terms are second order in the interactions. Both of those interactions act in very different scales. The self-interaction is short range and it was the case we studied in Sect. 3.6. The case of the gravitational interaction needs to be treated carefully since the gravitational interaction is long range. Here we follow Levkov et al. (2018) where the treatment from Landau for long-range Coulomb interactions was used for gravity.

For the initial Gaussian distribution, $F_{1}, F_{2}$ and $I(f)$ vanish, and the ULDM distribution is initially static.

We are interested in describing kinetic relaxation of ULDM in the halo of a galaxy (or in an axion minicluster), which is the condensation, and obtain the relaxation time of this process. As we saw before, inside the halo $y=\Delta x \ll R$, the field and the potential are homogeneous. In the center of a homogeneous spherically symmetric halo, where the condensate is formed (or a homogeneous box in the case of simulations), the terms $F_{1}$ and $F_{2}$ vanish and we do not need to take them into account when studying these initial stages of the condensate, where the four-wave kinetic equation is valid. Therefore, in our case

$$
\frac{\partial f}{\partial t}+\frac{\mathbf{p}}{m} \cdot \nabla_{x} f \approx I(f) .
$$

Lets first treat the gravitational part. To estimate $I_{\mathrm{G}}(f)$, we can follow Landau's treatment. In the regime where the above equation is valid $\Delta x \ll R$, and in the kinetic regime $(m v)^{-1} \ll R$, we can expand the potential as $U_{\mathrm{G}}(\mathbf{x}+\mathbf{y} / 2)=U_{\mathrm{G}}(\mathbf{x})+(\mathbf{y} / 2)$. $\nabla U_{\mathrm{G}}(\mathbf{x})$. With that the gravitational scattering integral we were calculating can be described by a diffusion process in phase space: $I_{\mathrm{G}}=-\nabla_{\mathbf{p}} \cdot \mathbf{s}$, where the Landau flux 
$\mathbf{s}$ is

$$
\begin{aligned}
\mathbf{s} & =\frac{1}{(2 \pi)^{3}} \int \mathrm{d} \mathbf{x}^{\prime} \mathrm{d} \mathbf{p}^{\prime} \mathcal{F}_{\mathbf{x} \mathbf{p}}^{\mathbf{x}^{\prime} \mathbf{p}^{\prime}} \nabla_{\mathbf{x}}\left(4 \pi G m^{2} \Delta_{\mathbf{x}-\mathbf{x}^{\prime}}^{-1}\right), \\
\mathcal{F}_{\mathbf{x p}}^{\mathbf{x}^{\prime} \mathbf{p}^{\prime}} & =\int \mathrm{d} \mathbf{y d} \mathbf{y}^{\prime} e^{-i\left(\mathbf{p} \cdot \mathbf{y}+\mathbf{p}^{\prime} \mathbf{y}^{\prime}\right)}\left\langle\psi_{+} \psi_{-}^{*} \psi_{+}^{\prime} \psi_{+}^{* \prime}\right\rangle_{\text {conn }} .
\end{aligned}
$$

Plugging this new $I_{\mathrm{G}}$ in the kinetic equation, we can rewrite it as a the equation for the evolution of the four-point function $\mathcal{F}$, which involves a six-point correlation function. To be able to write this equation in closed form and solve it, one uses Wick theorem. Ignoring the connected part in the limit of small separations $y \ll R$, the Landau flux is then $s_{i}=\int \mathrm{d} \mathbf{p}^{\prime} \Pi_{i j}(\mathbf{u})\left(f^{\prime 2} \partial_{p_{j}} f-f^{2} \partial_{p_{j}^{\prime}} f^{\prime}\right)$, where $\mathbf{u}=\left(\mathbf{p}^{\prime}-\mathbf{p}\right) / m$ and $f^{\prime}=f\left(\mathbf{x}^{\prime}, \mathbf{p}^{\prime}\right)$. The term $\Pi_{i j}(\mathbf{u})$ is given by the integral

$$
\Pi_{i j}(\mathbf{u})=\int \mathrm{d} t^{\prime} \mathrm{d} \mathbf{y} \partial_{i} \bar{\Delta}_{\mathbf{y}}^{-1} \partial_{j}\left[\bar{\Delta}_{\mathbf{y}+\mathbf{u} t^{\prime}}^{-1}-g \delta\left(\mathbf{y}+\mathbf{u} t^{\prime}\right)\right]
$$

where $\partial_{i} \bar{\Delta}_{\mathbf{y}}^{-1}=4 \pi G m^{2} \Delta_{\mathbf{y}}^{-1}$. Then this Landau flux defines $I_{\mathrm{G}}$ through $I_{\mathrm{G}}=-\nabla_{\mathbf{p}} \cdot \mathbf{s}$. However, to take the above integral one needs to introduce a long-time and a short-time cutoffs, given the logarithmic divergence of the Poisson Green's function. Therefore, the integral is taken in the interval $\left(m v^{2}\right)^{-} 1 \ll t^{\prime} \ll R / v$, which is the time that correspond to the regime where relaxation can happen since it can only happen inside the halo $y \ll R$ where the field is homogeneous, giving the upper limit, and for distances bigger than the de Broglie wavelength $y \gg(m v)^{-1}$, since diffusion is only sensitive to fluctuations at long distance (Kirkpatrick et al. 2020). The fact that diffusion is not sensitive to short distance scales already tells us that the self-interaction contribution be sub-dominant in the relaxation process. So the condensation in the halo happens much faster because of the gravitational interactions. In this time range, the dominant contribution of this integral yields $\Pi_{i j} \approx \Lambda\left(u^{2} \delta_{i j}-u_{i} u_{j}\right) / u^{3}$, where $\Lambda \equiv \log (m v R)$ is the Coulomb logarithm. We can compute the relaxation time due to gravity with this, which yields $I_{\mathrm{G}} \sim f / \tau_{\mathrm{G}}$.

The $I_{g}$ contribution is going to be sub-dominant, but we can still evaluate their contribution to the condensate. Since the self-interaction is short range, we do not need to resort to Landau's treatment. We can just set $U_{\text {tot }}=g|\psi|^{2}$, and solve Eq. (113). Considering again that we have a homogeneous distribution inside the halo and ignoring the connected part of the Wick theorem since we want the result in leading order of $g$, then the kinetic equation can be simplified to $\mathrm{d}^{2} f / \mathrm{d} t^{2} \sim 8 g^{2}\langle n\rangle f$, which tells us that the relaxation rate from the self-interactions is $\mathrm{d} I_{g} / \mathrm{d} t \sim 8 g^{2} n^{2} f=\gamma f$, where $\mathrm{d} / \mathrm{d} t=\partial_{t}+\mathbf{p} \cdot \nabla_{\mathbf{x}} / m$. This relaxation rate does not grow with time in this limit, but when the connected correlations start to become relevant the relaxation rate starts varying with time. The relaxation time from self-interactions is then given by $\tau_{g}=1 / \sqrt{\gamma}$. 
With that the relaxation time for this weakly interacting Bose gas subjected to gravity in a halo is given by

$$
\tau_{\mathrm{G}}=\frac{\sqrt{2} m v^{6}}{12 \pi^{3} G^{2} n^{2} \Lambda}, \quad \tau_{g}=\frac{1}{\sqrt{8}|g| n} .
$$

This gives the condensation time. The relaxation time for the gravitational interaction is inversely proportional to $G^{2}$ (and the cutoff scale), while the self-interaction one is inversely proportional to $g$. The gravitational relaxation time is much faster than the self-interaction one. The total condensation time is $\tau_{\text {tot }} \sim 2 \tau_{\mathrm{G}} \tau_{g} /\left(\tau_{g}+\sqrt{\tau_{g}^{2}+4 \tau_{\mathrm{G}}^{2}}\right) \rightarrow$ $\tau_{\mathrm{G}} \tau_{g} /\left(\tau_{\mathrm{G}}+\tau_{g}\right) \rightarrow \tau_{\mathrm{G}}$, is dominated by the gravitational one. Kirkpatrick et al. (2020) estimated this to be of the order $\tau_{g} / \tau_{\mathrm{G}} \sim 10^{5}$ for the QCD axion. Therefore, the formation of Bose stars mainly happens due to gravity, even in the presence of the selfinteractions. However, self-interaction is important as we saw above that the presence of the self-interactions lead to a different phenomenology and size of the soliton core. Notice that thermalization from gravitational interactions showed here is different then the one arising from short-range interactions through power-law turbulent cascades (Semikoz and Tkachev 1997), arising in this system through a diffusion process.

In the virialized halo, we can see that the formation of the Bose star, for the FDM and SIFDM models, can happen in the universe with formation time smaller than the age of the universe. For example, in dwarf galaxies, the condensation times for a FDM particle of mass $m_{22}$ would take $\sim 10^{6} \mathrm{yr}$.

This formalism shows us that we can describe the first stages of the formation of a BEC in the halo of galaxies using kinetic theory. This approximate description of the BEC can help us estimate the condensation time and study the properties of the Bose star. This formalism is particularly useful for numerical simulations which were performed in Levkov et al. (2018) for the FDM model. The formation of the condensate can be seen numerically with no seed condensate in the simulation. It is seen that the initial evolution of the condensate also follows Eq. (148) for $t<\tau_{\mathrm{G}}$, as expected for the wave turbulence with four-wave interaction. The Bose stars grow after formation and the condensate becomes stronger (more particles condensate). The first decade if this growth shows a growth in the mass of the Bose star $M_{s} \simeq c v_{0}\left(t / \tau_{\mathrm{G}}-1\right)^{1 / 2}$, with $c=3 \pm 0.7$. Only the first decade of the growth was seen both from computational limitation, but also this description does not hold for when the condensate starts to get stronger, and the description with four-wave interaction is expected to break.

That study shows us that we can indeed have the formation of a Bose-Einstein condensate of the ULDM particles inside the galaxies, forming Bose stars and solitons. This shows that the picture of having ULDM forming condensed cores inside galaxies is indeed valid. 


\subsubsection{Discussion}

Before going further and describing the observational consequences of the FDM and SIFDM, we are going to briefly discuss the picture presented here and some different interpretations regarding condensation that are present in the literature.

The picture that we have showed until now for the behaviour of the ULDM is the following. ULDM is described by a classical theory that gives its cosmological evolution and its non-relativistic evolution in galaxies. Inside the halos of galaxies, the ULDM thermalizes and forms gravitationally bound systems, the Bose stars or soliton, where a BEC or a superfluid is formed. This condensed core is smaller than the size of the galaxy and the coherent length gives the region where the wave-like behaviour of the condensate is manifested, being of the order of the de Broglie wavelength in the case of the FDM. The formation of these objects through gravitational interactions can occur inside the halo of galaxies, without any condensate seed, in a time smaller than the age of the universe, as shown from kinetic theory.

Therefore, in this picture, after the halo of the galaxy forms and virializes, thermalization in the inner regions of the halo happens and a condensate core is formed. This coherent length of the condensate, which changes if we are in the FDM or SIFDM cases, sets the size of the core that is smaller than the radius of the galaxy. This condensed core in the inner region of the galaxy is surrounded by a shell where DM behaves like a free particle ( since $\lambda_{\mathrm{dB}} \ll d$ ) instead of a wave like it is inside the core. Outside the cores, in the outskirts of the halo, DM follows the profile predicted by CDM. A condensate core can also be formed in the center of a more massive system like a cluster. However, this condensate is very small, smaller than the ones in galaxies, in comparison with the size of the cluster. Therefore, in the picture presented here, a $\mathrm{BEC}$ is formed in the interior of galaxies due to the gravitational interactions.

However, many authors in the literature there is a different view. For some authors, coherence of the ULDM is established initially, in the initial stages of formation of the ULDM. This is the case for axions, as pointed out in Sect. 4.7 of Marsh (2016a) (where a very good discussion of this topic is presented). The cosmic axion field is described as a classical coherently oscillating scalar field. This classical field $\phi$ comes from the expectation value of the quantum axion field, which can be represented in the Heisenberg picture as $\langle\phi|\hat{\phi}(x)| \phi\rangle=\phi(x)$. The state of the axion field $\phi$ is going to be coherent state (Zee 2003; Itzykson and Zuber 1980), with this coherence established initially. For the population of axions that is produced through vacuum misalignment, in the case where the Peccei-Quinn symmetry is broken during inflation, the inflationary evolution is responsible to making the axion coherent within the Hubble radius. For the axion population that forms via the decay of topological defects, coherence of the field is obtained via thermalization after formation with rate of the order of Hubble rate $H_{0}$, as presented in Marsh (2016a).

Therefore, as it is claimed in Marsh (2016a), the axions coming from any of the populations can be described cosmologically as a classical coherently oscillating scalar field. This classical description of the axion is said to hold throughout the evolution of the universe. This is discussed in Dvali and Zell (2018) where the validity of this mean field approximation is studied. They wanted to determine what is the quantum break time, which is defined as when quantum effects become important again for 
the description of the axion and the classical description of the axions is not valid anymore. The quantum breaking time is determined by the rate of axion scattering, and it is found that it exceeds the age of the universe for the QCD axion, but this would also be true for an axion with a smaller mass. Therefore, they conclude that it is safe to treat the axion as a classical coherently oscillating scalar field if coherence was established initially. ${ }^{34}$

This classical description also holds inside the halos of galaxies. This has been shown in Allali and Hertzberg (2020) for a ultra-light fields that behave like DM. The transition from a quantum to a classical system is called decoherence ${ }^{35}$. This is an important question since decoherence is known to occur very fast in macroscopic systems due to interactions of the system with the environment. However, if we have DM, where interactions with the environment are known to be very weak interacting mainly gravitationally, decoherence might proceed less efficiently. In this reference, they study decoherence of a ULDM, including axions, in the halo (ULDM overdensity) with its environment (the diffuse hydrogen in galaxies in this study). They find that ULDM in the halo can be treated as classical inside galaxies since decoherence would take place very fast, with a decoherence rate of the order of the Hubble rate $H_{0}$ for ULDM with $m \sim 10^{-7} \mathrm{eV}$. The same result holds for a BEC in the halo of galaxies. If we have a Bose star that formed in the halo, the decoherence time is very fast for any ULDM that has $m<\mathrm{eV}$, so the condensate, after formed, can be treated as classical and described by classical equations like the Schrödinger-Poisson system of equations.

With that, the picture that some authors have is that since coherence is established from initial conditions, and this classical picture can be maintained throughout the evolution of the universe, then when the Jeans length stable regions are formed, the axion in this region is already a coherent field. Thus, inside these regions in the interior of galaxies, coherence was established by initial conditions, so no thermalization is necessary to happen inside the halo.

However, the coherence of this classical field could be broken during the evolution of the universe by local processes. This can come from many out of equilibrium processes that occur in the universe. One example of this is the formation of halos since virialization happens through violent relaxation. In this case, the DM particles scatter on small fluctuations of the gravitational field, and coherence can be lost. The system can then be describe by an ensemble of classical incoherent waves, with very small coherence length. Also, as pointed out in Dvali and Zell (2018), even the

\footnotetext{
34 In this work, they consider that the axion is already represented by a classical coherent field, with all axions in the zero momentum. Thus, it already started with a classical uniform axion field. They do not discuss the thermalization process or any mechanism that led axions to this state. They only studied the maintenance and validity of the classical approximation.

35 Coherence in this reference is the term used to describe the pure quantum mechanical system. This notation comes from quantum mechanics since coherent states only occur in quantum mechanics. Therefore, it has a slight different meaning than the classical coherent scalar field we have been talking until now. The coherent classical field we have been talking until now is the field that came from the expectation value of the (quantum mechanical) field operator of this ULDM taken in these coherent states $\langle\phi|\hat{\phi}(x)| \phi\rangle=\phi(x)$. So we call this classical field of coherent classical field since the field was in a coherent state during its initial quantum stages. This term is usually used in the literature. Refer to Allali and Hertzberg (2020) for a proper definition of decoherence.
} 
classicality of the axion could be challenged in small overdense regions and quantum breaking could occur in these regions.

With all that, we can see that either having coherence from initial conditions or not, the ULDM field has to either thermalize or re-thermalize on galactic scales. We showed above that thermalization of ULDM can take place in the halos of galaxies in the presence of gravity or gravity and self-interaction, without any need of previous coherence of the field. And in these regions, it was shown that a BEC is going to be formed. And after its formation from the results from Allali and Hertzberg (2020) we can treat the evolution of the Bose star as classical. This picture is valid for the FDM, SIFDM and for the DM superfluid. So, for the phenomenology of ULDM in galaxies, if the field was already a coherent field or not, does not alter the conclusions we present in this review since this coherence can be reached at late times in the halo.

We just want to emphasize one last thing. Bose-Einstein condensation is a quantum mechanical phenomena. It can only arise because of quantum mechanics. The definition of a condensate is the one given in Sect. 3.7. A BEC can be described classically in the classical limit of the many-body theory or the field theory description of a condensate, or by an approximate classical theory as an ensemble of waves like wave turbulence (where we can make a parallel with the definition of a condensate as particles in the ground state which in the language of waves translates is waves with $k=0)$.

This discussion about thermalization or re-thermalization, classical or not, in the context of ULDM has been presented in many places in the literature of ULDM with many different point of views and interpretations. For the interested reader, some of these discussions are present in the following references (Sikivie and Yang 2009; Erken et al. 2012; Marsh 2016a; Guth et al. 2015; Castellanos et al. 2014; Davidson and Elmer 2013; Davidson 2015). We hope we have presented here a clear view of what happens in the interior of halos and how this can be interpreted, unifying some aspects of these interpretations.

\subsubsection{Cosmological and astrophysical consequences of the FDM}

Now that we have a description of what happens cosmologically and inside the halos of galaxies for these models, we are going to discuss the rich phenomenology that these models exhibit. We are going to study their predictions and discuss their cosmological and astrophysical consequences. These models behave differently than CDM in two ways. They present a CDM like behaviour on large-scales, with modified initial conditions, and inside the halos of galaxies, they form a core in the inner part of the halo where a non-CDM behaviour is described, while a NFW behaviour is present in the outskirts of the halo, outside the core (see Fig. 10).

We need to study the predictions coming from these modified initial conditions, and from the presence of these cores and their non-CDM behaviour. We can think about three main groups of consequences of these models. First is the suppression of the small-scale structure, that is going to affect many observables both cosmological and astrophysical; the second is related to the presence of the cores in the center of galaxies; and the third is related to dynamical effects that arise from the BEC or superfluid formed in the central regions of galaxies. We are going to describe these predictions in this 
section. We are going to focus mainly in the FDM in this section. Each of those effects can be probed with different observables, which can lead to bounds in the parameters of the models. We are going to talk about how these effects are measured and the constraints obtained in Sect. 5. For other observational consequences of FDM, see Hui et al. (2017).

A small comment. Although we showed above, we call solitons the ground state of the SIFDM, and Bose stars the ground state of the FDM, in the literature the term solitons is used for both. Therefore, from this point, we will use the term soliton to also describe the ground state of the FDM.

\section{Suppression of structure formation}

One of the effects coming from the FDM and SIFDM classes of models is the suppression of small-scale structure. This is a consequence of the fact that these models present sizable Jeans scale which cuts off the structure formation for wavelengths smaller than $\lambda_{\mathrm{J}}$. The Jeans length for the FDM model is given by Eq. (136). Therefore, as we saw in the previous section, for modes that are larger than the Jeans length $\lambda>\lambda_{\mathrm{J}}$, gravitational instability takes place and structure formation can happen, while modes smaller than the Jeans length $\lambda<\lambda_{\mathrm{J}}$ have oscillatory solution and no structure formation takes place. So structure formation suffers a cutoff on scales of the order of the Jeans length. The same happens for the SIFDM model, but the scale of this cutoff is different, analogous to the Jeans length, or the healing length, given by Eq. (127).

We can quantify this suppression by computing the power spectrum for the ULDM models. We are working in linear perturbation theory, as shown above, and we can evaluate the linear suppression of the power spectrum. In analogy to that done for the WDM model, the modifications of the power spectrum with respect to the $\Lambda \mathrm{CDM}$ power spectrum are encoded in a transfer function $T_{\mathrm{FDM}}(k, z)$. We can relate the power spectra as (Hu et al. 2000b; Marsh 2016a)

$$
P_{\mathrm{FDM}}(k, z)=T_{\mathrm{FDM}}^{2}(k, z) P_{\Lambda \mathrm{CDM}}(k, z)=T_{\mathrm{FDM}}^{2}(k, z)\left(\frac{D(z)}{D(0)}\right)^{2} P_{\Lambda \mathrm{CDM}}(k),
$$

where $P_{\Lambda \mathrm{CDM}}(k)$ is the power spectrum of $\Lambda \mathrm{CDM}$ at $z=0$ which in turn is the primordial power spectrum transformed by an appropriate transfer functions as defined in Bardeen et al. (1986); Eisenstein and Hu (1998); and $D(z)$ is the growth factor given by (Peebles 1993)

$$
D(z)=\frac{5 \Omega_{m}}{2 H(z)} \int_{0}^{a(z)} \frac{\mathrm{d} a^{\prime}}{\left(a^{\prime} H\left(a^{\prime}\right) / H_{0}\right)^{3}} .
$$

The FDM transfer function is given by

$$
T_{\mathrm{FDM}}=\frac{\cos \left(x_{\mathrm{J}(k)}^{3}\right)}{1+x_{\mathrm{J}}^{8}(k)}
$$


where

$$
x_{\mathrm{J}}(k)=1.61\left(\frac{m}{10^{-22} \mathrm{eV}}\right)^{1 / 18}\left(\frac{k}{k_{\mathrm{J}, \mathrm{eq}}}\right), \quad k_{\mathrm{J}, \mathrm{eq}}=9\left(\frac{m}{10^{-22} \mathrm{eV}}\right)^{1 / 2} \mathrm{Mpc}^{-1} .
$$

The scale $k_{\mathrm{J}, \text { eq }}$ is the Jeans length at matter-radiation equality, the time when perturbations start to grow.

This transfer function presents a fast decay at $k=k_{\mathrm{J}, \text { eq }}$, which leads to a suppression of the power spectrum on those scales. The mode where the power spectrum decays to half of its value $T\left(k_{1 / 2}\right)=1 / 2$ is the half mode given by Li et al. (2019) $k_{1 / 2}=$ $5.1\left(\mathrm{~m} / \mathrm{m}_{22}\right)^{4 / 9} \mathrm{Mpc}^{-1}$, where $m_{22}=10^{-22} \mathrm{eV}$. If $k_{1 / 2}<k_{n l} \sim 0.1$ where $k_{n l}$ is the non-linear scale which is around $1 \mathrm{Mpc}^{-1}$, the suppression of the power spectrum can be probed by linear CMB and LSS observables. Otherwise, we need non-linear observables. Therefore, roughly speaking, we can probe the suppression on the power spectrum on linear scales for FDM with masses $m \lesssim 10^{-23} \mathrm{eV}$. One interesting fact is that the $k_{1 / 2}$ of the FDM is the same as the one for WDM, although they are different functions of the wavenumber.

Beyond linear observables, this suppression of the power spectrum on small-scales induces a suppression in the formation of FDM halos. This can be estimated by calculating the linear half-radius $\left(R_{1 / 2}\right.$, lin $)$ which is the radius where half of the mass of the spherically symmetric system is contained. Then for $R_{1 / 2 \text {, lin }} \sim \lambda_{1 / 2} / 2$, where $\lambda_{1 / 2}=2 \pi / k_{1 / 2}$, we obtain the mass of the smallest halos that can be formed in this theory (Bullock and Boylan-Kolchin 2017; Niemeyer 2019)

$$
M_{\text {lin }}=\frac{4 \pi}{3} R_{1 / 2, \operatorname{lin}}\left\langle\rho_{\mathrm{FDM}}\right\rangle=4 \times 10^{10} M_{\odot}\left(\frac{m}{10^{-22} \mathrm{eV}}\right)^{3}\left(\frac{\Omega_{m}}{0.3}\right)\left(\frac{h}{0.7}\right)^{2}
$$

where $\left\langle\rho_{\mathrm{FDM}}\right\rangle=3 /(8 \pi G) H_{0}^{2} \Omega_{m}$. Therefore, FDM predicts a large suppression of halos for $M<10^{10} M_{\odot}$ if the mass is $m_{22}$. Below, we are going to see how this can be calculated more specifically given the cores in the halos and see how these predictions help address the small-scale problems.

This suppression of the power spectrum on small-scales also suppresses the formation of galaxies. It is found in simulations that the number of sub-halos in FDM in comparison to CDM is reduced by a factor of $\sim\left(3 M / M_{1 / 2}\right)^{2.4}$. This suppression of formation of small galaxies is larger in FDM at higher redshifts, in comparison to CDM. This opens up an important question about FDM being able to produce small-scale structures at early times to be probed by Lyman- $\alpha$ forest.

Therefore, the linear suppression of the power spectrum can affect both in the linear and non-linear part of the theory. This can lead to the following effects. This suppression can be probed by probing the linear power spectrum by the CMB and the matter power spectrum through LSS surveys. Or even better by observables that probe even lower scales, like Lyman- $\alpha$ forest and $21 \mathrm{~cm}$ from neutral hydrogen. The suppression of small halos also affects the non-linear scales. The substructure of this 
model is going to be different than in CDM, with a suppression of substructures on small-scales. The linear theory can predict a minimal mass for the structures formed, which can be probed by the population of satellites observed (and can be related to the missing satellites problem, as we will discuss in the next subsection). The substructures can also be probed directly by gravitational sensitive probes like gravitational lensing and streams, which are affected by the substructure present in the halo, and will be affected differently if the DM presents this suppression. We are going to see these observables and the bounds they can put in the models in Sect. 5. The substructures in the FDM model are also going to be changed by details of the presence of the core, which lead to different predictions that can be tested, as we will see below.

The entire numerical calculation of the power spectrum for the FDM model can be done using the software AxioncAmB (Lewis et al. 2000; Hložek et al. 2015) or a modification of software CLASS made in Ureña López and Gonzalez-Morales (2016). For our discussion in the next section, we used power spectra generated by AxionCAMB.

We discuss now the effect of the FDM in two observables that can probe this suppression, together with other phenomenology of the FDM like the change in the rate of expansion: the $\mathrm{CMB}$ and the matter power spectrum.

\section{CMB}

We want to review the modifications that FDM can cause in the observables of the CMB so we can understand how we can use this observation to probe the mass of the FDM. It is also possible to probe the fraction of FDM in the universe, if this is not assumed to be all the DM in the universe. We are interested in the case where FDM is all the DM in the universe, but we briefly comment here on the case where it is not.

The low mass of the FDM can alter the CMB in many different ways. We are mostly interested here in the effects in the primary $\mathrm{CMB}$ and $\mathrm{CMB}$ lensing, which are the ones that probe the DM in the ranges of mass we are interested for the ULDM models. There are other CMB observables that can probe other aspects of the microphysics of these models, and of their formation, as we can see in Fig. 2 from Abazajian et al. (2019).

ULDM affects the CMB in two main ways. ${ }^{36}$ The different expansion rate caused by the ULDM models affects the primary CMB, which is the adiabatic, unlensed without secondary effects CMB spectra. The suppression of the power spectrum that leads to a different clustering present in the ULDM models can be seen in the secondary lensing anisotropies.

We assume now that all the DM is given by ULDM. The primary CMB is affected by the expansion rate. Depending on the mass of the axion, their oscillations take place at different redshifts. If $z_{\text {osc }} \lesssim 1100$, then after recombination, the ultra-light field is behaving like dark energy and has a very different expansion rate than in the case of $\Lambda \mathrm{CDM}$. In this case, the amount of dark energy in the universe will be much bigger since this will be composed of $\Lambda$ plus the one from ULDM, affecting the first peak and the Sacks-Wolfe plateau (the plateau around $\ell \sim 100$ that can be seen in

\footnotetext{
36 Notice here that I am focusing only in the gravitational effects of the ULDM models. The CMB can also be affected by aspects related to the microphysics of some of these models and their formation mechanisms, which is specific to come models. For example, putting bounds in the axion isocurvature contributions can offer constraints in the axion decay constant $f_{a}$. It can even probe interactions in the dark sector. For more details on these other effects, check (Abazajian et al. 2019; Hložek et al. 2018).
} 
Fig. 11). The amount of the component that behaves as DM will be much smaller in this case, which in turn affects the other peaks. This can be seen in the left panel of Fig. 11. If the oscillations take place at $z_{\text {osc }} \gtrsim z_{\text {rec }} \approx 1100$, the ultra-light field behaves as DM before recombination, so they can alter the expansion rate during the radiation time. This affects the Silk damping tale, enhancing the higher acoustic peaks because of the reduction in the angular scale of the diffusion distance. This effect can be degenerate with changes in $N_{\text {eff }}$. As the mass gets heavier, which indicates that the ULDM behaves like DM since very early in the universe, the ULDM behaves more and more like CDM, and the angular power spectrum is very close to the $\Lambda \mathrm{CDM}$ one. ${ }^{37}$

In this figure, we plot the angular temperature power spectrum of coming from theoretical predictions of the FDM model for different masses, obtained using AxionCAMB. We also plot for comparison the data from the Planck $2018 \mathrm{Cl}$ TT power spectrum (Ade et al. 2016) and the $\Lambda \mathrm{CDM}$ model best fit to this data. We can see that for masses smaller than $10^{-25} \mathrm{eV}$ we can even visually see the deviation of the power spectrum from the data and from the $\Lambda \mathrm{CDM}$ one, with an enhancement of the size of all the peaks, and changing the relation between the second and third peaks, which indicates less DM with respect to baryons. For higher FDM masses, we cannot visually distinguish it from the $\Lambda \mathrm{CDM}$ one. Therefore, the primary $\mathrm{CMB}$ can put bounds on the mass of the ULDM according to their modified expansion rate at recombination.

Other effects might arise when the density of the axions is not equal to the total DM energy density. There is a degeneracy between the amount of ULDM, $\Omega_{\text {ULDM }}$, and the amount of curvature (or dark energy) and matter. This can be seen in more detail in Marsh (2016a).

The ULDM can also affect the secondary lensing anisotropies. The small-scale suppression of the power spectrum can be seen as a lensing deflection power on scales $\ell>1000$. This can be seen in the lensing convergence power spectrum. This effect from the suppression of clustering can also be seen in the matter power spectrum, as we show below. This effect is degenerate to the one coming from massive neutrinos. For smaller mass ULDM, one can use the effects of expansion to break this degeneracy, but this is not the case in the range of masses we are interested for ULDM that has an important effect on small-scales.

This shows that CMB is a powerful observable to probe many aspects of ultra-light particles, specially in the low-mass range. We are going to see in Sect. 5 the constraints obtained by CMB observations in the FDM model and discuss some forecasts.

\section{Matter power spectrum}

The matter power spectrum brings information about the matter density contrast in the universe with respect to the scale. The matter power spectrum contains a huge amount of information. Considering the full shape of the power spectrum, we can measured the equality scale $\left(k_{\text {eq }}^{-1}\right)$ which sets and can be inferred by the peak of the matter power spectrum (a bit hard to see in right panel of Fig. 11) and its overall shape. The other feature that is present in the power spectrum are the BAO features, from where we can obtain the BAO frequency and infer the sound horizon at baryon drag (at $z_{\text {rec }}$ ).

\footnotetext{
37 In this section, where we are treating the FDM model, when I say heavier FDM masses I mean $m \gtrsim$ $10^{-25} \mathrm{eV}$.
} 

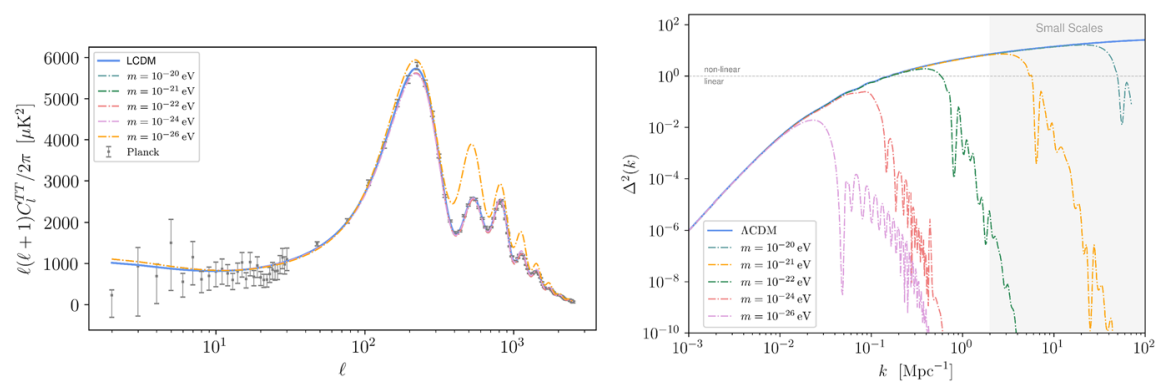

Fig. 11 Left panel: angular power spectrum of the temperature CMB anisotropy for the FDM model for different masses where FDM is considered all the DM in the universe. We compare this with the $\Lambda$ CDM model best fit to the Planck data (Ade et al. 2016) showed by the gray data points. Right panel: matter power spectrum for the same FDM model as used in the left panel

The ULDM affect the matter power spectrum both by having a different expansion rate and from the suppression of clustering on small scales. A different expansion rate would alter the BAO, presenting a different sound horizon in the power spectrum. This is more relevant for smaller masses of the FDM particle, when the FDM behaves like dark energy for a longer time in the evolution of the universe.

The other effect which is more dramatic on small-scales is coming from the different clustering that the FDM presents. As we can see in right panel of Fig. 11, for a FDM that represents all the DM in the universe, the matter power spectrum for FDM presents a suppression of the small-scale power spectrum. As we saw, this suppression comes from the Jeans length of the FDM that suppresses the power spectrum via the transfer function. We can see that the smaller the mass is, the effect is more dramatic. For masses that affect the linear scales, this can be easily tested using observations. However, we can see that for the heavier FDM particles, this suppression occurs on the small-scales, where there is not a lot of observational data.

We can use galaxy surveys to probe the biased matter power spectrum. This can give us bounds in the mass of the FDM, as we will see in Sect. 5. However, for heavier masses we can see that the suppression in the power spectrum occur on smaller scales, not probed by galaxy surveys. For that we need new observables, like Lyman- $\alpha$ which allows us to probe scales of order $0.5 \mathrm{Mpc} / h \lesssim \lambda \lesssim 100 \mathrm{Mpc} / h$. This permits to constrain higher masses for the FDM. Another window of observation that allows us to probe the smaller scales is $21 \mathrm{~cm}$ from neutral hydrogen, which can gives the matter power spectrum on scale $k>10 \mathrm{Mpc}^{-1}$. We will discuss these observables in Sect. 5 .

One important point about using the power spectrum is the issue of the bias. To infer the matter power spectrum one needs to observe biased tracers of the DM distribution. The bias relates each of those tracers with the underlying DM distribution. This bias for each tracer is unknown, and each probe presents a different bias. Therefore, when obtaining constraints on the mass of the FDM, there is a degeneracy with bias. There is also the possibility of a scale dependence in the bias of ULDM. This is still a not so well-studied problem for the ULDM, with a few studies from numerical simulations for the FDM case (Cooray and Sheth 2002; Hložek et al. 2015). One can also obtain the matter power spectrum from shear measurements, coming from gravitational lensing. 
This are unbiased tracers and can provide measurements of the FDM mass with a complementary approach.

\section{Halo density profile}

We saw above that the suppression of the power spectrum in the FDM model leads to a suppression in formation of small mass halos. Only halos with $M>M_{\text {lin }}$ where $M_{\text {lin }}$ is given by (160) are formed in the FDM. This suppression can lead to a different number of low mass halos, modifying the halo mass function.

The halo mass function (HMF) describes the density of halos per unit of mass. To determine the HMF of the FDM one needs to resort to either simulations or semianalytic methods. Usually simulations are performed and the HMF can be fitted, as done in Schive et al. (2016). In this reference they have a simulation of collisionless particles with initial conditions coming from the FDM. The fitted HMF obtained is given by:

$$
\left(\frac{\mathrm{d} n}{\mathrm{~d} M}\right)_{\mathrm{FDM}}=\left[1+\left(\frac{M}{M_{0}}\right)^{-1.1}\right]^{-2.2}\left(\frac{\mathrm{d} n}{\mathrm{~d} M}\right)_{\mathrm{CDM}}
$$

This HMF presents a suppression for low mass halos, characterized by the scale $M_{0}=1.6 \times 10^{10} m_{22}^{-4 / 3}$. The HMF of CDM depends on the redshift and mass of the halo, while the suppression term in brackets is redshift independent, which is a consequence of the FDM modification only coming from the initial conditions. This means that this also does not take into account the effective sound speed of the FDM, only through the suppression of the initial power spectrum. This HMF is very accurate for higher masses, in agreement with the CDM HMF, but it presents an uncertainty in the low-mass end, showing that this HMF is reliable to show the suppression on those scales, but not so reliable to obtain the slope of the HMF for FDM for low halo masses.

There is also another HMF obtained using different methods. in Marsh and Silk (2014) with the aim of taking into account the scale dependent linear growth from the FDM, they obtain the HMF from a modified Press-Schechter approach. This yields

$$
\left(\frac{\mathrm{d} n}{\mathrm{~d} M}\right)_{\mathrm{FDM}}=-\frac{\rho_{m}}{M} f(v) \frac{\mathrm{d} \ln \sigma^{2}}{\mathrm{~d} \ln M},
$$

where $v \equiv \delta_{c} / \sigma, \delta_{c}$ is the critical collapse overdensity and $\sigma(M)$ is the variance of the power spectrum. The variance is calculated by smoothing the power spectrum with a spherical top-hat window function. The function $f(v)$ comes from the Sheth-Thormen model (Sheth and Tormen 1999) and it is given by $f(v)=A(q / 2 \pi)^{1 / 2} \nu\left[1+(q v)^{(-}\right.$ $2 p)] \exp \left(-q v^{2} / 2\right)$, with parameters $A=0.3222, p=0.3$ and $q=0.707$. The critical overdensity $\delta_{c}$ brings more details about the evolution of the FDM. At $z=0$, it can be described by comparing $\delta_{c}^{\mathrm{FDM}}(k)=\mathcal{G}(k) \delta_{c}$, where $\delta_{c}=\delta_{c}\left(z=0, k=k_{0}\right)$ and $k_{0}=0.002$ is the pivot scale. The scale-dependent function $\mathcal{G}(k)$ is the ratio of the scale-dependent growth factors from $\Lambda$ CDM and FDM (Marsh and Silk 2014; Bozek et al. 2015) (in Marsh (2016b) a numerical fitting for this function was found). There are many other modifications of this HMF and alternative formulations that can be 
seen in Sheth and Tormen (1999), Bozek et al. (2015), Du et al. (2017b) and Schneider (2015).

We are now going to see how this prediction can be probed by the luminosity function and reionization.

\section{Luminosity function and reionization}

Observations that probe the low-mass end of the halo mass function can be used to test the FDM and put constraints in the mass. A sensitive probes is the luminosity function of galaxies, which can inherit the suppression coming from the halo mass function. Observations at high redshifts of galaxy counts, reionization history can be used to test the suppression of the HMF caused by the FDM.

The luminosity function $\phi(L)$ is a map between the galaxy luminosity and the dark matter halo. If we want to obtain cumulative galaxy number density so that we can compare with observations, we need to relate the UV magnitude of a galaxy with the mass of the halo. If there are less halos formed at early times, this would lead to less galaxy formation. This can be one characteristic of the FDM model to be tested, but this suppression cannot be too severe otherwise this model could lead to less than expected high- $z$ galaxies than seen in observations, like the ones from the Hubble Ultra Deep Field (Bouwens et al. 2015). This lack of galaxies could also impact the efficiency of the reionization of the intergalactic medium (IGM), which takes place through star formation. This would impact the optical depth of the CMB, which is constrained by CMB observations (Ade et al. 2016).

The relation between the HMF and the luminosity function is usually done by abundance matching (Kravtsov et al. 2004; Vale and Ostriker 2004; Conroy et al. 2006). For that, one needs to assume a mapping between the halo mass and the galaxy luminosity or the UV magnitude of the galaxy. This is done by matching the cumulative UV luminosity function $\Phi(L, z)$ for magnitude smaller than $M_{\mathrm{UV}}$ (which is the same as luminosities higher than $L_{\mathrm{UV}}$ ) given by the integral of $\phi(L)$ in this interval, with the cumulative HMF for halos masses bigger than a given $M_{h}: \Phi\left(<M_{\mathrm{UV}}, z\right)=n(>$ $\left.M_{h}, z\right)$. That, together with fixing $\phi(L)$ at low-z with observations, fixes the mass to luminosity (or UV magnitude) of galaxies.

Given the modified HMF presented by the FDM model, we can then predict the luminosity function of this model. In Schive et al. (2016) and Bozek et al. (2015), we can see some examples of the luminosity function for FDM. The low-mass suppression in the HMF leads to a luminosity function that ends at smaller magnitudes. Therefore, depending on the mass of the FDM particle, we can predict a cumulative luminosity function that ends at different magnitudes. This can be used to put constraints in the FDM using, for example, observations of dwarf galaxies or measurements of high- $z$ galaxies like the one from the HUDF. We discuss this in Sect. 5.

Depending on the mass of the FDM particle we can predict less galaxies at high$z$, which would alter the reionization history. This can be seen given that the UV luminosity function is related to the flux of ionizing photons (Niemeyer 2019),

$$
\mathcal{F}_{\text {ion }}=f_{\text {esc }} \int \phi_{U V}(L) \gamma(L) \mathrm{d} L,
$$

where $f_{\text {esc }}$ is the scape fraction, related to the fraction of ionizing photons that escapes the galaxy without being absorbed, and $\gamma(L)$ the conversion rate, which describes the 
conversion between the UV luminosity of galaxies to the luminosity of ionizing photon (for more details see Schive et al. 2016 and references therein). With that we can write the Thomson optical depth to CMB:

$$
\tau=\int_{0}^{z} \mathrm{~d} z^{\prime} \frac{\left(1+z^{\prime}\right)^{2}}{H\left(z^{\prime}\right)} Q\left(z^{\prime}\right) \sigma_{T} \bar{n}_{H}\left(1+\eta_{\mathrm{He}} Y / 4 X\right),
$$

where $\sigma_{T}$ is the Thomson cross section, $\bar{n}$ is the mean comoving hydrogen number density, $X$ is the hydrogen fraction, $Y=X-1$ is the helium fraction, and $\eta_{\mathrm{He}}$ is the ionization state of helium.

We can then use this modified reionization history, coming from the modified luminosity function, to constrain the mass of the FDM. One can use, for example, the CMB where $\tau\left(z_{\text {rec }}\right)$ is measured.

\section{Sub-halo mass function}

As discussed above, the FDM model is characterized by a suppression in the formation of the small structures. This minimal mass of structures formed is going to impact the substructures in the halo. Therefore, a smaller number of sub-halos is present in the FDM in comparison to CDM. This suppression can be seen in the sub-halo mass function, which is given by $\mathrm{d} n_{\text {sub }}(m) / \mathrm{d} \ln M$, where $n_{\text {sub }}$ is the number of sub-halos and $M$ is the halo mass. This can also be obtained from simulations and semi-analytic calculations (Schive et al. 2016; Corasaniti et al. 2017; Marsh and Silk 2014; Du et al. 2017a), where a fitting form for the FDM sub-halo mass function is obtained (Du 2018; Schutz 2020):

$$
\left(\frac{\mathrm{d} n_{\mathrm{sub}}}{\mathrm{d} \ln M}\right)_{\mathrm{FDM}}=f_{1}(M)+f_{2}(M)\left(\frac{\mathrm{d} n_{\mathrm{sub}}}{\mathrm{d} \ln M}\right)_{\mathrm{CDM}},
$$

where the functions present in this fitting formula are given by

$$
\begin{aligned}
& f_{1}(M)=\beta \exp \left[-\frac{1}{\sigma}\left(\ln \frac{M}{M_{1} \times 10^{8} M_{\odot}}\right)^{2}\right], \\
& f_{2}(M)=\left[1+\left(\frac{M}{M_{2} \times 10^{8} M_{\odot}}\right)^{-\alpha_{1}}\right]^{-10 / \alpha_{1}} .
\end{aligned}
$$

The influence of tidal stripping of cores in the sub-halo mass function is studied in Du et al. (2018), showing that core stripping influences this function for sub-halo masses smaller than $10^{7} M_{\odot}$. Including this effect the parameters in (165) are $\alpha_{1}=0.72$, $\sigma=1.4, \beta=0.014 \mathrm{~m}_{22}^{3 / 2}, M_{1}=4.7 \mathrm{~m}_{22}^{3 / 2}$, and $M_{2}=2.0 m_{22}^{1.6}$. For these parameters, this fitting formula agrees with simulations for masses smaller than $5 \times 10^{-21} \mathrm{eV}$.

In Fig. 12, we compare the sub-halo mass function for FDM with different masses with the CDM one. Here we used the following CDM sub-halo mass function:

$$
\left(\frac{\mathrm{d} n_{\mathrm{sub}}}{\mathrm{d} \ln M}\right)_{\mathrm{CDM}}=a_{\mathrm{CDM}}\left(\frac{M}{10^{8} M_{\odot}}\right)^{-\alpha_{0}} .
$$




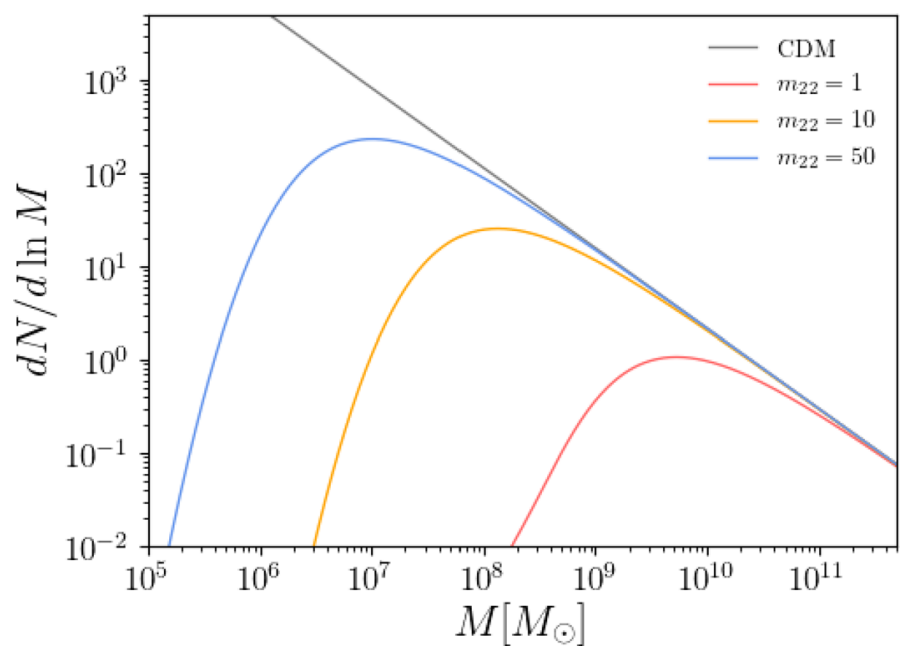

Fig. 12 Comparison between the sub-halo mass function from CDM and FDM for different masses of the FDM particle. In the case of the FDM, we consider that all DM is given by the FDM model

where $a_{\mathrm{CDM}}=113.094$ and $\alpha_{0}=0.86$, which was obtained as a fit to the subhalos of the Aquarium simulation (Springel et al. 2008). We can see in the figure the suppression of the small-scale structures by the redshift dependent cut in the sub-halos mass function for smaller sub-halo masses, characteristic of the FDM model.

With the sub-halo mass function expected for the FDM model, one can test if the predictions from this model are in accordance with observations.

\section{The central soliton}

We have shown in Sect. 4.1.4 that there is the formation of a soliton (or Bose star) in the central parts of the galaxy, which is the ground state solution of the SchrödingerPoisson system. However, there is no analytical solution for the soliton and we have to obtain this solution numerically. This was done by many authors (Hui et al. 2017; Chavanis 2011; Schive et al. 2014a, b; Kaup 1968; Harrison et al. 2002; Ruffini and Bonazzola 1969a; Guzmán and Ureña-López 2004; Bar-Or et al. 2019), and here we are going to quote the numerical solutions obtained by Schive et al. (2014a, b). Here we will restore the $\hbar$ factors for clarity.

An interesting characteristic of the Schrödinger-Poisson is that tit has scaling symmetry, which allows to re-scale the quantities of this problem by an arbitrary variable $\beta$ as $\{t, x, \Phi, \phi\} \rightarrow\left\{\beta^{-2} t, \beta^{-1} x, \beta^{2} \Phi, \beta^{2} \phi\right\}$ (Ji and Sin 1994; Guzmán and UreñaLópez 2006). This is also valid for the SIFDM, although more subtle (Guzmán and Ureña-López 2006), and the interaction term scales as $g \rightarrow \beta^{-2} g$. The solutions also obey this scaling transformation, and the physical quantities transform as: the energy density of the soliton transforms as $\rho_{s} \rightarrow \beta^{4} \rho_{s}$, the radius as $r \rightarrow \beta^{-1} r$, and the mass of the soliton as $M_{s} \rightarrow \beta M_{s}$. This means that one can simple re-scale the solution to the equilibrium scale of interested, like the virial scale, if interested in the cores in galaxies, or study the axion star in QCD axion miniclusters (Niemeyer 2019). 
By assuming spherical symmetry, we need only to determine one of those physical quantities, like the soliton density, and we can derive the other parameters from it.

The density of the soliton core can be approximated by (Hui et al. 2017): $\rho_{c} \simeq$ $4 \times 10^{-3}\left(G m^{2} / \hbar^{2}\right)^{3} M^{4} \simeq(3 M / 4) R_{1 / 2, c}^{-3}$ where the half-mass radius is $R_{1 / 2, c} \bar{\rho}_{1 / 2} \simeq$ $4 \hbar^{2} / G M m^{2}$, the radius where the density drops to one half of its value ${ }^{38}$. This can be re-written as (Schive et al. 2014a, b)

$$
\rho_{c} \simeq \frac{1.9 \times 10^{-2}}{\left[1+0.091\left(r / R_{1 / 2, c}\right)^{2}\right]^{8}}\left(\frac{m}{10^{-22} \mathrm{eV}}\right)^{-2}\left(\frac{r_{c}}{\mathrm{kpc}}\right)^{-4} M_{\odot} \mathrm{pc}^{-3},
$$

With the density, we can compute the soliton mass

$$
M_{c}=4 \pi \int_{0}^{\infty} r^{2} \rho_{c}(r) \mathrm{d} r=2.2 \times 10^{8}\left(\frac{m}{10^{-22} \mathrm{eV}}\right)^{-2}\left(\frac{R_{1 / 2, c}}{\mathrm{kpc}}\right)^{-1} M_{\odot} .
$$

Here we are approximating these expression by considering that $R_{1 / 2, c}=r_{c}$ is the radius of the condensate, and the mass of the soliton core is the mass enclosed in the sphere with this radius. We can see that $95 \%$ of the soliton mass is within $r \leq 3 r_{c}$, and this means that (168) is accurate to $2 \%$ for $0 \leq r \leq r_{c}$.

We are interested in studying the soliton cores formed inside the galaxies, so we use the scaling to obtain the solution soliton radius in a virial halo of MW-like mass

$$
r_{c} \simeq 0.16\left(\frac{m}{10^{-22} \mathrm{eV}}\right)^{-1}\left(\frac{M}{10^{12} M_{\odot}}\right)^{-1 / 3} \mathrm{kpc} .
$$

With that we can see that the FDM soliton core for a FDM with mass $m=10^{-22} \mathrm{eV}$ is going to be smaller than the halo of the MW, with size $r_{c} \sim 0.16 \mathrm{kpc}$ in the center region of the halo. In the outskirts of the halo, FDM is expected to behave as free particles, like CDM, so the core is enveloped by shell with in NFW-profile:

$$
\rho_{\text {halo }} \simeq \begin{cases}\rho_{c} & \text { for } r \leq r_{c} \\ \rho_{\mathrm{NFW}} & \text { for } r>r_{c}\end{cases}
$$

which is the picture we showed in Fig. 10. We use this simplified picture to describe what happens in the halo. We can see that the soliton core is inside the Jeans length, as expected, if one compare with Eq. (136). But it is of the order of the de Broglie wavelength if we use the velocity in the soliton $v_{c}^{2}=r /\left(G M_{c}\right)$,:

$$
\lambda_{d B}\left(v=v_{c}\right)=3.91 r_{c}\left(\frac{r}{r_{c}}\right)^{1 / 2} .
$$

In Schive et al. (2014b) based on the scaling of the Schorinder-Poisson system and using the result of simulations it was also derived the core-halo mass relation for the

\footnotetext{
38 Notice that this is the half-radius of the soliton core, since it is the radius where the core density drops in half. This is different than the half-radius of the halo, for example.
} 
FDM, showing that $M_{c} \propto(1+z)^{1 / 2} M^{1 / 3}$, where $M$ is the halo mass. This shows that MW like halos with $M \sim 10^{12} M_{\odot}$ at a redshift around $z \sim 8$ will have soliton cores forming after the halo collapse with mass $M_{c} \sim 10^{9} M_{\odot}$. These soliton cores can be seen in FDM simulations, as we will see in Sect. 4.3.

The presence of these soliton cores in the interior of the galaxies lead to a rich phenomenology. Since no gravitational clustering happens in these regions, the soliton core has a cored profile $\rho_{c}(r)$, which is different than the one expected from CDM. Therefore, if we can probe the central density of galaxies and obtain a non-cored profile, we can use this to put a bound on the FDM mass. Dwarf galaxies can also be used to do that. Since those galaxies are DM dominated, one can measure their central density and compare with the bound obtained by the expected core to constrain the mass of the FDM. We are going to discuss this in detail in Sect. 5, and also in a extreme case in Sect. 4.1.7, where we discuss how the core can solve the cusp-core problem.

\section{Dynamical effects-relaxation}

In this section, we are going to discuss the third class of observational effects of the ULDM, the dynamical effects. These are effects that arise because of the wave-like behaviour that ULDM have inside the soliton cores in galaxies. This can modify the dynamics of objects that are present inside this region where condensation takes place. We are going to see here two of those effects that arise from the relaxation between the FDM and macroscopic objects that move in or through the condensed core. These effects can lead either to heating, which is seen as an energy injection in the orbit of the macroscopic object, or to cooling or dynamical friction, where orbit of the object loses energy to the FDM field.

These two effects are present in CDM and other DM models, but are modified here by the wave-like behaviour of the FDM. Effects like an enhanced heating can lead to observational effects such as the increase in the velocity dispersion of systems and their expansion. On the other hand, this modified dynamical friction can be used to explain effects like system that were expected to have merged but did not, which represents a challenge for the CDM paradigm.

In the same way that we studied relaxation between the FDM particles leading to the formation of a BEC in Sect. 4.1.4, we can use the same techniques of kinetic theory to study the relaxation of the FDM and the macroscopic objects. We describe below briefly this analysis. This was introduced in Bar-Or et al. (2019) and the reader should consult this reference for more details. There are also different ways to model dynamical friction, which we will comment below.

We want to describe the relaxation between the FDM and macroscopic objects inside the condensate core. Therefore, we can think about this as a test particle, or a contaminant, that is present in the condensate ${ }^{39}$. We want to see how stochastic density fluctuations in the FDM core lead to diffusion of the velocity of the macroscopic object $^{40}$.

Using kinetic theory one can treat the problem as a diffusion of a test particle in a fluctuating density field. In the case of the FDM, the FDM is represented by a

\footnotetext{
39 We are going to see this modelled from a microscopic theory in Sect. 4.2.4 in the case of the superfluid.

40 In this section, I restore the $\hbar$ factors since the relation with the classical limit is more subtle. See Bar-Or et al. (2019) for more details.
} 
wavefunction which is a collection of plane waves, $\psi(\mathbf{r}, t)=\int \mathrm{d} \mathbf{k} \varphi(\mathbf{k}) \exp (i \mathbf{k} . \mathbf{r}-$ $i \omega(k) t)$, with $\omega=\hbar^{2} k^{2} / 2 m$, interacting with a zero-mass particle. In this case, we can ignore gravitational interactions between the FDM particles and only focus in the interaction of the FDM and the classical test particle. The FDM can be treat in this way inside the core where it is condensed.

Computing the diffusion coefficients for this system of a test particle in the FDM condensate, it was found that it yields the same diffusion coefficients as the ones for a classical two-body relaxation where the test particle interacts with a homogeneous classical density field composed by particles with mass $m_{p}$, but with a different effective mass for the FDM "particles" $m_{\text {eff }}$, a different distribution function $F_{\text {eff }}$ and a different Coulomb logarithm $\log \Lambda_{\mathrm{FDM}}$ :

$$
m_{\mathrm{eff}}=\frac{(2 \pi \hbar)^{3}}{m^{3}} \frac{\int \mathrm{d} \mathbf{v} f^{2}(\mathbf{v})}{\int \mathrm{d} \mathbf{v} f(\mathbf{v})}, \quad f_{\mathrm{eff}}=f^{2}(\mathbf{v}) \frac{\int \mathrm{d} \mathbf{v} f(\mathbf{v})}{\int \mathrm{d} \mathbf{v} f^{2}(\mathbf{v})},
$$

where $f(\mathrm{v})$ is the FDM distribution function and the effective distribution function is normalized like $\rho_{\text {FDM }}=\int \mathrm{d} \mathbf{v} f_{\text {eff }}(\mathbf{v})$. The Coulomb logarithm is $\log \Lambda_{\text {FDM }} \approx$ $\log \left(2 b_{\max } / \lambda_{\mathrm{dB}}(\sigma)\right)$, where $b_{\max }$ is the maximum scale of encounter and $\sigma$ is the dispersion velocity.

So the picture is that for understanding the effects of a macroscopic object in the FDM core, one can think as the FDM was composed by effective particles with mass $m_{\text {eff }}$, called FDM "quasiparticles" in Bar-Or et al. (2019) ${ }^{41}$. If this quasiparticle has a mass that is larger than the mass of these macroscopic objects $m_{t}$, this leads to the object to either lose energy or otherwise to gain energy from the encounters with these quasiparticles. These two processes are the cooling (or dynamical friction) and heating.

Thus, if $m_{\text {eff }} \gg m_{t}$, the fluctuations in the FDM field inject energy into the orbit of the macroscopic object. This injection increases the velocity dispersion of the macroscopic object, which in turn increases its size and we have heating. The heating time scale is defined as

$$
\tau_{\text {heat }}=\frac{3 \sigma^{3}}{16 \sqrt{\pi} G^{2} \rho_{\mathrm{b}} m_{\mathrm{eff}} \log \Lambda_{\mathrm{FDM}}}=\frac{3 m^{3} \sigma^{6}}{16 \pi^{2} G^{2} \rho_{\mathrm{b}}^{2} \hbar^{3} \log \Lambda_{\mathrm{FDM}}}
$$

For times smaller than $\tau_{\text {heat }}$, the system can gain energy from these FDM fluctuations and heating takes place. For values of the effective mass in halos, we can see that the quasiparticle is more massive than stars. This heating of stars either in a cluster or in the disk of galaxies can lead to observational effects.

If $m_{\mathrm{eff}} \ll m_{t}$, then the mass of the macroscopic object is bigger than the FDM quasiparticle, and the macroscopic object loses energy from its orbit to the FDM field. This process can be interpreted as dynamical friction. Since the macroscopic object is

\footnotetext{
41 Careful here that the term quasiparticle was used for the phonon and they do not have the same meaning as here.
} 
losing energy, this process is also known as cooling, with a cooling time given by

$$
\tau_{\mathrm{cool}}=\frac{3 \sigma^{3}}{8 \sqrt{2 \pi} G^{2} \rho_{\mathrm{b}} m_{\mathrm{t}} \log \Lambda_{\mathrm{FDM}}}
$$

This process usually happen for more massive objects like globular clusters merging with the center of the galaxy or black holes.

A similar analysis is done in El-Zant et al. (2016, 2020b), where they confirm the results from Bar-Or et al. (2019) and extend the model to include the effect density perturbations coming from stellar winds, supernova explosions or active galactic nuclei. The consequence for super massive black holes in a FDM halo was studied in El-Zant et al. (2020a) which can lead to its ejection from the core. This effect can be used to put bounds in the FDM mass.

We are going to discuss below some specific examples where these dynamical effects take place.

\section{Gravitational heating}

We can now see here how the heating affects a stellar population. We show this for a prototype case of a spherical stellar system of radius $r_{\star}$ in a FDM gravitational potential. The initial distribution function of the stellar system is Maxwellian with a velocity dispersion $\sigma_{t}$. Since star have $m_{t}=m_{\text {star }} \ll m_{\text {eff }}$, only heating takes place.

The heating is important when $\tau_{\text {heat }, \star}$ is of order of $1 / 3$ of the age of the galaxy $\tau_{\text {age }}$, where

$$
\tau_{\text {heat }, \star} \approx \frac{2.08}{\log \Lambda_{\mathrm{FDM}}}\left(\frac{r_{\star}}{1 \mathrm{kpc}}\right)^{4}\left(\frac{m}{10^{-22 \mathrm{eV}}}\right)^{3}\left(\frac{m}{200 \mathrm{~km} / \mathrm{s}}\right)^{2} \mathrm{Gyr} .
$$

In this case, we have a increase in the velocity dispersion and the system is going to expand $^{42}$. This happens in scales when $r_{\star}<r_{\text {heat }, \star}$ with

$$
r_{\text {heat }, \star}=1.13 \log \Lambda_{\mathrm{FDM}}\left(\frac{\tau_{\text {age }}}{10 \mathrm{Gyr}}\right)^{1 / 4}\left(\frac{m}{10^{-22 \mathrm{eV}}}\right)^{-3 / 4}\left(\frac{m}{200 \mathrm{~km} / \mathrm{s}}\right)^{-1 / 2} \mathrm{kpc} .
$$

For the comparison with observation that we are going to show in Sect. 5, we are interested in the cases where this effect leads to a increase in the dispersion relation of the star, expanding the stellar system. This is going to be studied in the disks of galaxies and also in a star cluster, like Eridanus II.

\section{Dynamical friction}

The change in dynamical friction is one of the most interesting consequence in BECs and superfluids. This emergent phenomenology can lead to consequent observations

\footnotetext{
42 One thing to be attentive here is that heating or diffusion is the term used for the injection of energy in the orbit of the macroscopic system by the FDM. This might cause an increase in the velocity dispersion, which leads to an expansion of the system, or not depending on the radial profile of the galaxy (see Bar-Or et al. 2019 for more details).
} 
that might reveal the characteristics of those systems. It is interesting to see how dynamical friction behaves in the presence of a BEC core, as in the case of the FDM. The dynamical friction in a BEC is not expected to change as dramatically as in a superfluid, which has no friction, but some change is expected nevertheless.

It is expected that the FDM changes this prediction because of three phenomena: (1) change in the rate of orbital decay because of the presence of the condensed core; (2) since the FDM produces a homogeneous core, a mechanism similar to the "core stalling" observed in N-body simulations can take place and reduce or eliminate drag from dynamical friction; and (3) the way dynamical friction is calculated must be modified by the presence of an object with large de Broglie wavelength, a quantum mechanical extension to the calculation of dynamical friction must be done.

An interesting puzzle that can potentially be explained by a modified dynamical friction is the puzzle of the Fornax globular clusters. From the standard dynamical friction expected for CDM and baryons, it is expected that globular clusters orbiting Fornax should have rapidly fallen towards its center to form a stellar nucleus. However, there is no signal of mergers and we detect 5 globular clusters orbiting Fornax.

In Hui et al. (2017), only the last effect is described and simulated for different parameters the orbital decay times for Fornax in CDM and in the FDM cases. They found that in FDM the orbital decay time is longer, and four of the five decay times simulated are bigger than $10 \mathrm{Gyr}$ or more, thus explaining the puzzle for why the globular cluster in Fornax survived. More simulations and observations are needed to confirm this claim, but the FDM model seems to address the dynamical friction puzzle. The ideal is to have the microscopic theory describing dissipation in the FDM model.

These qualitative results are confirmed in Bar-Or et al. (2019) using the classical two-body relaxation, shown above. See also results for how dynamical friction alters inspiral systems (Bar-Or et al. 2019).

Finally, in Lancaster et al. (2020), a detailed analytical and numerical study of dynamical friction in the FDM model was performed. To describe the dynamical friction in this model, they describe the dissipation that a perturber moving in a condensate causes. They work this dissipation theory for point-sources (satellites), extended satellites and point-like satellite in a FDM background with finite velocity dispersion. This analytical theory is then verified by their numerical-simulation that solves the Schrödinger-Poisson system in the presence of such perturber satellite, showing good agreement with the analytic methods. This framework is applied to the cases of the Fornax globular cluster, but also to the Sagittarius (Sgr) stream and the Large Magellanic Clouds (LMC) (we will see about those systems in more detail in Sect. 5). For the Fornax, they find that if the mass of the FDM model is $m \gtrsim 10^{-21} \mathrm{eV}$ this model stops explaining the Fornax globular cluster merging times, which is in agreement with the mass bounds necessary for the FDM to solve the small-scale problems. For Sgr and LMC it is found that the dynamical friction on those are described by the classical limit, described by the Chandrasekhar formula. More simulations need to be done to confirm this, and it is very important to understand the dynamical friction in this regime since these bodies have a strong influence in the MW, and are the target of many studies and observations. These results already show that this modified dynamical friction in 
the FDM model can maybe explain some interesting astrophysical observations which might be a good opportunity to measure the ULDM on galactic scales.

\section{Clusters: most massive halos}

The FDM can also affect clusters. For distances larger than the de Broglie wavelength of the FDM, it is expected that DM behaves as standard CDM and that the halo enveloping the soliton has a NFW profile. This can be seen numerically for the mass range $10^{9} M_{\odot} \lesssim M_{\text {vir }} \lesssim 10^{11} M_{\odot}$ (Schive et al. 2014a, b; Schwabe et al. 2016; Veltmaat and Niemeyer 2016), which gives an estimate for the mass of the central soliton. Extending this relation to larger halos, the FDM predicts that in the inner regions of clusters there will be a condensed core, a soliton, with mass

$$
M \simeq 1.3 \times 10^{10} M_{\odot}\left(\frac{10^{-22}}{m}\right)\left(\frac{M_{\mathrm{vir}}}{10^{15} M_{\odot}}\right)^{1 / 3}
$$

which is still below the maximum mass for the soliton calculated above for a galaxy. The corresponding half-mass radius is

$$
R_{1 / 2} \simeq 25 \mathrm{pc}\left(\frac{10^{-22}}{m}\right)\left(\frac{M_{\mathrm{vir}}}{10^{15} M_{\odot}}\right)^{-1 / 3}
$$

So, the presence of this soliton with this mass and size would be a prediction of the FDM model. But a question that remains to be answered is the following: is the presence of such solitons in the interior of clusters halos compatible with merging cluster like the Bullet cluster or the anti-Bullet cluster?

In Hui et al. (2017), they ask the question if solitons in the center of the galaxies have not been misinterpreted as super massive black holes. They compare the mass of the central dark region measured from Virgo and show that this is similar to the mass of the soliton core in a galaxy like Virgo for a mass of the FDM particle of $m \sim 10^{-22} \mathrm{eV}$. However, since the observations from the Event Horizon Telescope of the black hole in the center of M87 were released, this hypothesis seem to be almost excluded, and it is indeed a super massive black hole that inhabits the center of this galaxy. We have to wait for more data to confirm this, but this is an exciting measurement that can also be used to test the FDM hypothesis.

Another interesting fact is that we know that the galaxies host a super massive black hole in their center. For this reason in Hui et al. (2017) they investigate the possibility of a super massive black hole to be created in the center of a soliton. Apparently, the black holes do not grow for the fiducial mass $m_{22}$, in a condensate core. Their creation only starts being significant for $m \gtrsim 5 \times 10^{-22} \mathrm{eV}$, which is in tension with other bounds in the mass, like the one to solve the missing satellites problems.

\subsubsection{Addressing the small-scale challenges}

Perhaps even more interesting than solving the small-scale problems of the CDM paradigm, it is the new and rich phenomenology that the ULDM present. But the 
ULDM models can also address these small-scale challenges and reconcile the smallscale observations with the CDM paradigm. Usually the scales where the non-CDM and interesting phenomenology happens coincides with the scales where these models present modifications in the small-scales that are necessary to explain the small-scale observations.

We are going to discuss now how this new phenomenology on small scales presented by the FDM can address the small-scale challenges and what are the conditions in the parameter of this class of models for that to happen. We are only going too discuss the FDM case now, and the conditions in the mass, but one can think that a similar analysis can be done for the case of SIFDM.

\section{$F D M$}

We want to determine the mass of the FDM candidate. From the discussion above, we saw the there is a bound for the mass to condensate in galaxies $10^{-25} \lesssim m \lesssim \mathrm{eV}$ for a typical MW-like galaxy. Later we saw that, for masses of order of the usual QCD axion mass, around $m \sim 10^{-5} \mathrm{eV}$, the stable configurations are very localized and small, far from galactic scales. With that we can already see that $m \ll 10^{-5} \mathrm{eV}$ for the FDM model. Now, we are going to see other conditions that can bound the mass and show the mass range for the FDM particle that can address the small-scale challenges.

\section{- Halos: minimum size, maximum density and the cusp-core problems:}

The general idea why FDM (and all ULDM models) can solve the cusp-core problem is because these systems naturally predict a core in the center of galaxies. In this core there is no structure formation (Jeans instability), and for that reason they might prevent the formation of a cusp in the center of the galaxy. We are going to investigate here how and for which mass the FDM core size and density can address the cusp-core problem.

Since we want the non-CDM behaviour to happen inside galaxies, the de Broglie wavelength of the FDM inside galaxies cannot exceed the size of the galaxy, given by the virial radius: $\lambda_{\mathrm{d}} B<R=G M / v^{2}$. Therefore, $R \gtrsim \frac{1}{G M m^{2}}$, where $M$ is the mass of the galaxy. We can write that in terms of the half-radius, radius where half of the mass of spherically symmetric system is contained (Hui et al. 2017)

$$
R_{1 / 2} \gtrsim 0.335\left(\frac{M}{10^{9} M_{\odot}}\right)^{-1}\left(\frac{m}{10^{-22} \mathrm{eV}}\right)^{-2} \mathrm{kpc} .
$$

This bound on the radius is compatible with the half-light radii inferred from the densities of 36 Local group dwarf spheroidals (McConnachie 2012) if the mass of the FDM particle is $m \sim 10^{-22} \mathrm{eV}$.

With the above condition, we can also compute the upper bound in the central density:

$$
\rho_{\mathrm{c}} \leq 7.05\left(\frac{M}{10^{9} M_{\odot}}\right)^{4}\left(\frac{m}{10^{-22} \mathrm{eV}}\right)^{6} M_{\odot} \mathrm{pc}^{-3}
$$

If we compare this bound to the observations from 8 dwarf spheroidals, and we can see for the density to be comparable to the one measured for these $\mathrm{dSph}$, the 
FDM mass needs to be $m=8_{-3}^{+5} \times 10^{-23} \mathrm{eV}$ for Draco and $m=6_{-2}^{+7} \times 10^{-22} \mathrm{eV}$ for Sextans (Chen et al. 2017). For those masses, the distribution at the center of the galaxies seem to be cored, alleviating the cusp-core problem.

Therefore, it is necessary a mass of order $m \sim 10^{-22} \mathrm{eV}$ in order for FDM to solve the cusp core problem. And we showed that this is compatible with the measurements from dwarf galaxies. However, we are going to discuss in Sect. 5.2.1 that some studies dispute this conclusion.

- Lower bound on the FDM halo masses, and the missing satellites and too big to fail problem:

As we saw before, for the self-gravitating FDM systems, since gravity is attractive, we have coherence on a finite scale. The size of this core depends on the mass, being larger as the mass gets smaller. So the smallest radius to be produced in the FDM model are determined by the smallest mass allowed for the particle.

Having a limit for the smallest cores that can be created has important consequences in the abundance of low mass halos, and it is going to be different in this model than what is given by $\Lambda \mathrm{CDM}$. We can see that by calculating the smallest structures formed in the FDM model. This is given when $\lambda=\lambda_{\mathrm{J}}$, where $\lambda_{\mathrm{J}}$ represents the last scales that can suffer gravitational instability. With the Jeans length, we can calculate the Jeans mass:

$$
\begin{aligned}
M_{\mathrm{J}} & =\frac{4 \pi}{3} \rho\left(\frac{1}{2} \lambda_{\mathrm{J}}\right)^{3}=1.5 \times 10^{7} M_{\odot}(1+z)^{3 / 4}\left(\frac{\Omega_{\mathrm{FDM}}}{0.27}\right)\left(\frac{H}{70 \mathrm{~km} / \mathrm{s} / \mathrm{Mpc}}\right)^{1 / 2} \\
& \left(\frac{10^{-22} \mathrm{eV}}{m}\right)^{3 / 2}
\end{aligned}
$$

This is the minimum mass of substructure created in the FDM model. This is in contrast with CDM, where halos with mass below $\sim 10^{8} M_{\odot}$ are highly created, with abundance $\mathrm{d} n\left(M_{\mathrm{h}}\right) \propto M_{\mathrm{h}}^{-2} \mathrm{~d} M_{\mathrm{h}}$. In that sense, the missing satellites problem is addressed in the FDM model, since halos of smaller masses than $M_{\mathrm{J}}$ are not created, if $m=10^{-22} \mathrm{eV}$. If the mass of the FDM is smaller than that, subhalos with smaller mass will form, and the FDM model is not going to address the missing satellite problem anymore. Therefore this shows that to solve this problem, the FDM has a preferred mass of around $10^{-22} \mathrm{eV}$. We are going to see below that tidal disruption can also act suppressing small mass halos, aiding FDM in solving the missing satellites problem. The too big to fail problem is also addressed by the FDM, since we have a mechanism to explain the fact that low-mass sub-halos are not formed, making it not necessary to invoke mechanisms that creates the too-big-to-fail problem as a by product.

Summarizing, to address the small-scale challenges, which means presenting a cored density profile inside galaxies and suppressing the formation of small mass sub-halos which would explain the smaller amount of satellites, the mass of the FDM needs to be of the order of $\sim 10^{-22} \mathrm{eV}$. A component with that mass and presenting the dynamics of the FDM class of models would behave like CDM on large-scales and present the observed structures on small-scales. 
However, we are going to see in Sect. 5 that, although for many years this mass range was available as a possibility for the mass of the FDM, the latest observations have been challenging this mass, and showing that the FDM has to probably have a heavier mass than the one necessary to address the small-scale problems.

\subsection{DM superfluid}

In this section, we are going to describe the third category of models of ULDM, the DM superfluid. In previous sections we saw the small-scale problems of $\Lambda \mathrm{CDM}$ and how MOND empirical law offered a very good fit to the rotation curves of galaxies and the scaling relations that emerge from the dynamics of galaxies, which might be challenging in the context of $\Lambda \mathrm{CDM}$. However, as we saw, there is no present framework that can explain MOND, given that the initial proposed theory, the full MOND, and its extensions present serious problems. We present here an alternative model to DM that has the goal of reconciling CDM and MOND: the DM superfluid. This model intends to not only solve the problems that the previous models attempt to address but also offer a mechanism to describe MOND on small-scales. In this framework, DM behaves as standard CDM on large-scales, while the MOND dynamics emerges on galactic scales. And this is possible through the physics of superfluidity.

The idea of the DM superfluid model is that DM forms a superfluid on galactic scales, where superfluidity arises upon condensation. This superfluid core present in the inner regions of galaxies not only addresses the small-scale challenges of $\Lambda \mathrm{CDM}$ in a similar way as the previous models, but the superfluid described in these regions behaves following a different dynamics which reproduces the MOND behaviour. This is possible given that this superfluid is described by a Lagrangian similar to thee one from the MOND theory which is allowed given the EFT description of the superfluid. In this way, the long-range correlation present in MOND is going to be given by the behaviour of the phonons which mediate long-range forces. Outside the superfluid core, DM behaves as normal matter as in the previous models. This is the general vision how the DM superfluid model attempts to describe the behaviour of DM in galaxies.

In the following subsection, we will construct the DM superfluid theory showing first in which conditions DM condensates on galactic scales, following that we will present the theory that describes this superfluid phase. With that in hand we can calculate the halo profile and rotation curves to compare with data and check the fit of the theory. We present how this model explain many astrophysical systems and possible predictions. After that we show the limits of validity of this description and its relativistic completion. We briefly describe how the cosmology works in this model.

This model is constructed using the fact that in a generic superfluid we can reproduce the exact action as in MOND. This is a very specific model and serves as a toy model for the understanding of theories of DM that present an emergent dynamics on smallscales. It is important to point out that what is important is for the model to be able to explain the rotation curves and scaling relations described by galaxies. This translates into a theory that exhibits long-range correlation on Galactic scales. We present here a very specific example of a model when this occurs, where the way the long-range 
correlation is obtained is by restricting the Lagrangian to behave like MOND on smallscales, but this does not have to be the case. The search for a more general theory where this emerges, with a known microphysics is the final goal. Such a construction is currently being searched.

\subsubsection{Conditions for DM condensation}

Before describing how the DM superfluid behaves inside galaxies, we need to determine in which conditions DM condensates into a Bose-Einstein condensate in galaxies. As we saw in the previous section, two conditions need to be met for condensation: first, we need that all the particles are in a single coherent quantum state, described by a single wavefunction of the condensate; a second condition is that the DM particles are in thermal equilibrium, to be described by a Bose distribution.

In this section, we want to obtain a rough estimate of the bounds in the parameters of the model to obtain this condensed core in the inner parts of the galaxy. For that, for simplicity, we use the criteria for weakly interacting gases.

\section{Condensate wavefunction and thermalization}

We showed in the beginning of this section, the condition on the mass for the ULDM particles to condense in galaxies, showing that they should be in the range $10^{-25} \mathrm{eV} \lesssim m \lesssim 2 \mathrm{eV}$. This is an approximate condition for the case of DM in galaxies, but it gives us an order of magnitude estimation for the mass of the DM superfluid particles. However, if we remember from Sect. 3 , there is a second condition for condensation.

The second condition to form a condensate is that the particles are in thermal equilibrium. The condition to achieve thermal equilibrium is that the time scale of thermalization must be smaller than the time scale where dynamical processes happen in the halo, the dynamical time. If this condition is satisfied and thermal equilibrium is achieved, the condensate is coherent in the entire halo. The time scale of thermalization if given by the inverse of the self-interaction rate, and the condition for thermalization is given by

$$
\Gamma \sim \Upsilon v_{\text {vir }} \rho_{\text {vir }} \frac{\sigma}{m} \lesssim t_{\text {dyn }}^{-1}=(3 \pi / 32 G \rho)^{-1 / 2}
$$

where $\Upsilon \sim \frac{\rho_{\mathrm{vir}}}{m} \frac{(2 \pi)^{3}}{(4 \pi / 3)(m v)^{3}}$ is the Bose enhancement factor, which tells you if a boson is already in the state, the probability to another boson to be in that state will be enhanced by a factor of $\Upsilon$. The dynamical time is taken here as the time it takes to a sphere of density $\rho$ to collapse due to gravity. This condition gives a bound in the self-interaction cross section:

$$
\frac{\sigma}{m} \gtrsim 0.3\left(\frac{m}{\mathrm{eV}}\right)^{4} \frac{\mathrm{cm}^{2}}{g},
$$

where we assumed $z_{\text {vir }}=2$ and $M=10^{12} M_{\odot}$. If we want that our self-interaction satisfies the merging-cluster bound (Randall et al. 2008; Massey et al. 2011; Harvey 

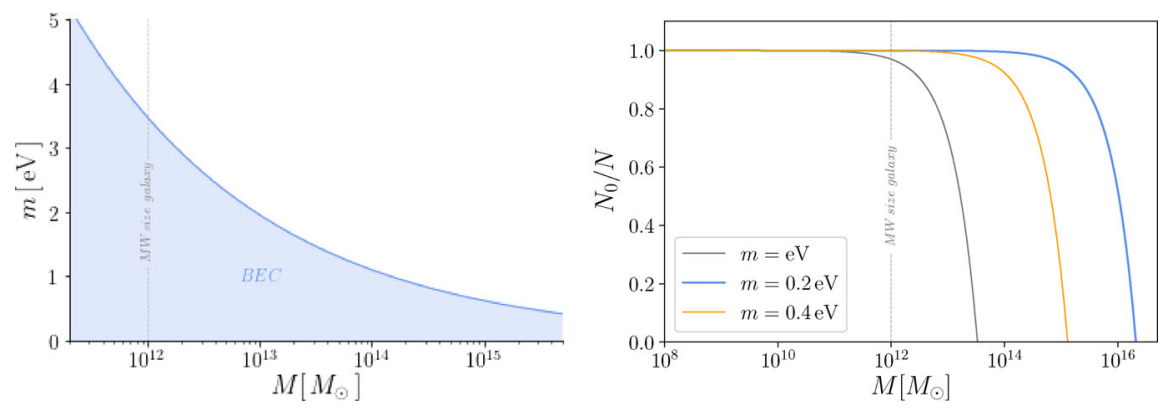

Fig. 13 Left panel: mass of the superfluid particle with respect to the mass of the halo. The filled are represents the regime where DM is condensed and behaves as a superfluid, for $z_{\mathrm{vir}}=2$. Right panel: approximate calculation form (Berezhiani and Khoury 2015) for the fraction of the particles in the condensate versus in the normal state, for a series of sub eV masses in accordance with our bounds. We assumed $z_{\mathrm{vir}}=0$

et al. 2015), which is $\sim 1 \mathrm{~cm}^{2} / \mathrm{g}$, this gives another bound in mass of the superfluid: $m \lesssim \mathrm{eV}$.

From these conditions, we can obtain a few properties of the our DM superfluid condensate:

- Critical temperature: With DM in thermal equilibrium, the temperature can be obtained by the equipartition theorem: $k_{\mathrm{B}} T=m\langle v\rangle^{2} / 2$, which is valid for temperatures smaller than the critical temperature. Above that temperature, the condensate is broken. The critical temperature $T_{\mathrm{c}}$ is associated with the "critical" velocity $v_{\mathrm{c}}$, than can be read when we saturate the bound (90):

$$
T_{\mathrm{c}} \sim 6.5\left(\frac{\mathrm{eV}}{m}\right)^{5 / 3}\left(1+z_{\mathrm{vir}}\right)^{2} \mathrm{mK}
$$

With that the temperature in a halo of mass $M$ is given by:

$$
\frac{T}{T_{\mathrm{c}}} \sim 0.1\left(1+z_{\mathrm{vir}}\right)^{-1}\left(\frac{m}{\mathrm{eV}}\right)^{8 / 3}\left(\frac{M}{10^{12} M_{\odot}}\right)^{2 / 3}
$$

- Condensate fraction: As we saw on Sect. 3 at $T=0$ it is expected that almost all the particles are in the condensate. However, at finite but subcritical temperature, as seen in Landau's theory (Landau and Lifshitz 1980), it is expected that the fluid is going to be a mixture of superfluid and normal fluid, with the majority in the superfluid. Borrowing from the non-interacting BEC description, this can be estimated as

$$
\frac{N_{\text {cond }}}{N}=1-\left(\frac{T}{T_{\mathrm{c}}}\right)^{3 / 2} \sim 1-0.03\left(1+z_{\mathrm{vir}}\right)^{-3 / 2}\left(\frac{m}{\mathrm{eV}}\right)^{4}\left(\frac{M}{10^{12} M_{\odot}}\right) .
$$

This formula is only valid for free-particles, and a particle with interaction and trapped in the gravitational potential has a different power than $3 / 2$, but it serves as 
an estimate. We can see in Fig. 13 the condensate fraction with respect to the mass of the halo for different masses of the DM particle. From this, we an see that for a MW-like galaxy for any mass smaller than $\mathrm{eV}$, the particles are in the condensate, while for higher masses of clusters, for example, this is not true. Therefore, the mass range from the bound (88) describes a condensate that condenses on galactic scales.

The above conditions were obtained assuming that the condensate will take the entire halo. However, as mentioned in Sect. 2.1, virialization occurs through violent relaxation, which is an out-of-equilibrium process. In this way, the DM superfluid cannot thermalize. What should happen is that first, the halo virialized and the profile is the expected NFW. After this process, DM particles start to enter thermal equilibrium in the inner, most central regions of the condensate, where the interaction is more pronounced. In this way, the halo would have an inner region $\left(r<R_{T}\right)$ where DM is in a condensed state surrounded by the outer part of the halo $\left(r>R_{T}\right)$ that follows the NFW profile (Berezhiani et al. 2018). Since in this model the goal is to be able to describe the rotation curves of galaxies, $R_{T}$ needs to be larger than the radius where the circular motion of stars and gas is observed. For $r>R_{\mathrm{T}}$, the density profile of the halo follows the NFW profile, $\rho \propto r^{-3}$. So we can rewrite the density and velocity with respect to the virial quantities used above: $\rho(r)=\rho\left(R_{200}\right)\left(R_{200} / r\right)^{3}$, where for a NFW we can estimate $\rho_{200} / \rho\left(R_{200}\right) \sim 5$. With that, the thermalization bound becomes:

$$
\frac{\sigma}{m} \gtrsim 0.2\left(\frac{m}{\mathrm{eV}}\right)^{4}\left(\frac{M}{10^{12} M_{\odot}}\right)^{2 / 3}\left(\frac{r}{R_{200}}\right)^{7 / 2} \frac{\mathrm{cm}^{2}}{g},
$$

which tells us that it is easier to reach thermal equilibrium in the center of the galaxies where the density is higher. This translates into a bound to the thermalization radius:

$$
R_{T} \lesssim 310\left(\frac{m}{\mathrm{eV}}\right)^{-8 / 7}\left(\frac{M}{10^{12} M_{\odot}}\right)^{1 / 7}\left(\frac{\sigma / m}{\mathrm{~cm}^{2} / g}\right)^{2 / 7} \mathrm{kpc}
$$

For a MW-like galaxy with $M=10^{12} M_{\odot}$, if we can measure the the circular velocity up to approximately $60 \mathrm{kpc}$, this will translate into a bound for the mass: $m \lesssim 4.2\left(\frac{\sigma / m}{\mathrm{~cm}^{2} / g}\right)^{1 / 4} \mathrm{eV}$

\subsubsection{Superfluid dynamics}

Since we have determined that DM condenses and forms a superfluidity in the central regions of the halo, we now need to describe the evolution of this superfluid inside this region. We need to determine the dynamics of the superfluid to be able to calculate the profile of the region of the halo comprising the superfluid and with the calculate the rotation curves of galaxies. In this section, we will describe the effective field theory of superfluids and show how this is theory reproduces MOND at small scales. 
As we saw in the previous section, a superfluid at low-energies is described by the effective Lagrangian that is invariant under shift and Galilean symmetries:

$$
\mathcal{L}_{T=0}=P(X), \quad X=\dot{\theta}+\mu-m \Phi-\frac{(\nabla \theta)^{2}}{2 m}
$$

where $\Phi=-G M(r) / r$ is the external gravitational potential for a spherical symmetric static source. The thermodynamic pressure is given by $P$.

We want our theory to describe the MOND dynamics at the regions where it is superfluid. Given this general Lagrangian for the phonons (190), we want it to describe the MOND Lagrangian (8). For this, we conjecture that our phonon action is given by

$$
\mathcal{L}_{\mathrm{DM}, T=0}=\frac{2 \Lambda(2 m)^{3 / 2}}{3} X \sqrt{|X|} .
$$

This fractional power might seem strange from the point of view of a quantum field theory of fundamental fields, leading to superluminal behaviour and caustics. However, as a theory for the phonons, this is not problematic and it determines uniquely the equation of state of the superfluid. As we can see for the condensate, the background, where $\theta=\mu t$, the pressure is given by the Lagrangian density:

$$
P(\mu)=\frac{2 \Lambda}{3}(2 m \mu)^{3 / 2}, \quad \Rightarrow \quad P=\frac{\rho^{3}}{12 \Lambda^{2} m^{6}}
$$

where, in the non-relativistic regime, $\rho=m n$ and $n=\partial P / \partial \mu$ is the number density of condensed particles. As expected from the result from MOND, this Lagrangian gives us an EoS for the superfluid $P \propto \rho^{3}$, which is what we wanted to reproduce MOND.

To evaluate the excitation spectrum, we write the action for the phonon excitations $\phi$, that can be obtained by expanding (190) to quadratic order. Neglecting the gravitational potential:

$$
\mathcal{L}^{(2)}=\frac{(2 m)^{3 / 2}}{4 \mu^{1 / 2}}\left(\dot{\phi}^{2}-\frac{2 \mu}{m}(\nabla \phi)^{2}\right),
$$

from where we can infer the sound speed of the phonon excitations:

$$
c_{\mathrm{s}}=\sqrt{\frac{2 \mu}{m}} \text {. }
$$

However, only those ingredients are not enough to reproduce a MOND-like force and a coupling between the phonons and the baryon density needs to be introduced:

$$
\mathcal{L}_{\mathrm{int}}=-\alpha \frac{\Lambda}{M_{\mathrm{pl}}} \theta \rho_{\mathrm{b}}
$$


where $\alpha$ is a dimensionless coupling constant. Although necessary to obtain the MOND regime, this interaction Lagrangian breaks shift symmetry softly, only at the $1 / M_{\mathrm{pl}}$ level. This term is here considered as a phenomenological term to reproduce MOND. In this way, this superfluid theory has three parameters: the mass $m$, the scale $\Lambda$ and the coupling $\alpha$.

The present form of the Lagrangian to obtain MOND is not the only way of obtaining the MOND behavior in the context of the DM superfluid model. In Khoury (2016) it was used higher order corrections to generate the non-relativistic MOND action, which is inspired in the symmetron mechanism. Using the same Lagrangian (191) as the leading order Lagrangian, higher order corrections involving gradients of the gravitational potential are added to effectively modify the gravitational force. This results in the spontaneous breaking of a discrete symmetry. The symmetry is broken for small accelerations leading to MONDian gravity, and is restored in the limit of large acceleration leading to Newtonian gravity. In this theory the shift symmetry of the entire system is maintained. A difference from the present mechanism, as we are going to see later, is that cosmologically all the DM is in the normal phase, behaving like CDM, and reproducing all the results from $\Lambda$ CDM. Here we will describe the method of adding a photon-baryon coupling since this was studied in more detail in the literature.

\section{Finite temperature}

The theory developed above is valid for a $T=0$ superfluid. However, in reality, the DM in galaxies has a non-zero temperature. As we mentioned in Sect. 3, for finite temperatures, this Lagrangian needs to receive finite temperature corrections. In Landau's model the finite temperature superfluid consists is described as a two-fluid theory where a superfluid component and a normal component are present. Those components must interact with each other. Following this idea from Landau's theory, at lowest order in derivatives, we can write the general form of the EFT at finite temperatures and finite chemical potential as a function of three scalars (Nicolis 2011):

$$
\mathcal{L}_{T \neq 0}=F(X, B, Y)
$$

where $X=X(\theta)$ was defined before with respect to the superfluid variables. The other new components are: $B$ is defined with respect to the normal fluid three Lagrangian coordinates $\varphi^{I}(\mathbf{x}, t)$; and $Y$ represents the scalar product of the normal and superfluid velocities:

$$
B \equiv \sqrt{\operatorname{det} \partial_{\mu} \varphi^{I} \partial^{\mu} \varphi^{I}}, \quad Y \equiv u^{\mu}\left(\partial_{\mu} \theta+m \delta_{\mu}^{0}\right) \simeq \mu-m \Phi+\dot{\phi}+\mathbf{v} \cdot \nabla \phi,
$$

where $u^{\mu}$ is the unit 4-vector from $\varphi^{I}(\mathbf{x}, t)$, and in the last equality of $Y$ we have taken the non-relativistic limit, so $\mathbf{v}$ is the velocity vector of the normal fluid component.

There are many ways to construct the finite temperature operators. Our restriction is that we want our finite-temperature theory to generate the expected MOND profile. To construct such a Lagrangian requires first-principle knowledge of the microphysics of the superfluid. Since we still do not have a fundamental description of the DM superfluid model, we proceed empirically. We suggest the following finite-temperature 
Lagrangian for the model:

$$
\mathcal{L}=\frac{2 \Lambda(2 m)^{3 / 2}}{3} X \sqrt{|X-\beta Y|}-\alpha \frac{\Lambda}{M_{\mathrm{pl}}} \phi \rho_{\mathrm{b}},
$$

where the finite temperature effects are parametrized by a dimensionless constant $\beta$. When $\beta \rightarrow 0$, we recover the $T=0$ result; we are using the fiducial value $\beta=2$. We included the interaction term so we could represent the entire action of the model that we are going to use next.

\subsubsection{Halo profile}

With the Lagrangian of the theory, we can evaluate the halo profile in the superfluid region and, after matching with an outer NFW profile, calculate the rotation curves of galaxies. And this is what we are going to do in this section: estimate the halo profile. This will be done in steps. First, we estimate the DM halo profile taking into account only the density coming from (191). Next, we include the baryons, by calculating the profile for the full action including interaction. We are going to use here the finitetemperature effective action (198) since in the case of the $T=0$ the perturbations around a static background configuration suffer from a ghost-like instability. Although phenomenological, it retains the features of the initial superfluid Lagrangian and can give a more realistic description of the system.

\section{DM halo profile}

We can now calculate the density profile of the condensate, in the superfluid region, assuming that we have only dark matter and no baryons for simplicity. This is the halo profile given by the different equation of state that the superfluid has: $P \propto n^{3}$, given by equation (192). This analysis is almost the same for the zero-temperature and finite temperature cases, with accounts for the replacement: $\Lambda \rightarrow \tilde{\Lambda}=\Lambda \sqrt{\beta-1}$. Assuming hydrostatic equilibrium, for a static and spherically symmetric halo, the pressure and acceleration are related by:

$$
\frac{1}{\rho(r)} \frac{\mathrm{d} P(r)}{\mathrm{d} r}=-\frac{\mathrm{d} \Phi(r)}{\mathrm{d} r}=-\frac{4 \pi G}{r^{2}} \int_{0}^{r} \mathrm{~d} r^{\prime} r^{\prime 2} \rho\left(r^{\prime}\right) .
$$

By making a change of variables $\rho(r)=\rho_{0} \Xi$ and $r=\xi\left[\rho_{0} /\left(32 \pi G \tilde{\Lambda}^{2} m^{6}\right)\right]^{1 / 2}$, where $\rho(r=0)=\rho_{0}$, this equation reduces to the Lane-Emden equation (with $n=1 / 2)$,

$$
\frac{1}{\xi^{2}} \frac{\mathrm{d}}{\mathrm{d} \xi}\left(\xi^{2} \frac{\mathrm{d} \Xi}{\mathrm{d} \xi}\right)=-\Xi^{1 / 2}
$$

Choosing boundary conditions $\Xi(0)=1$ and $\Xi^{\prime}(0)=0$, we can numerically solve this equation. We can see from the change of variables that the size of the condensate 
and the central density are given by Chandrasekhar (1967),

$$
R=\xi_{1} \sqrt{\frac{\rho_{0}}{32 \pi G \tilde{\Lambda}^{2} m^{6}}}, \quad \rho_{0}=\frac{M}{4 \pi R^{3}} \frac{\xi_{1}}{\left|\Xi^{\prime}\left(\xi_{1}\right)\right|},
$$

where at $\xi_{1}$ the numerical solution vanishes. From the numerics $\xi_{1} \sim 2.75$ and $\Xi^{\prime}\left(\xi_{1}\right) \sim-0.5$ gives the following halo radius and central density:

$$
\begin{aligned}
\rho_{0} & \sim\left(\frac{M_{\mathrm{DM}}}{10^{12} M_{\odot}}\right)^{2 / 5}\left(\frac{m}{\mathrm{eV}}\right)^{18 / 5}\left(\frac{\Lambda}{\mathrm{meV}}\right)^{6 / 5}(\beta-1)^{3 / 5} 10^{-24} \mathrm{~g} / \mathrm{cm}^{3}, \\
R & \sim\left(\frac{M_{\mathrm{DM}}}{10^{12} M_{\odot}}\right)^{1 / 5}\left(\frac{m}{\mathrm{eV}}\right)^{-6 / 5}\left(\frac{\Lambda}{\mathrm{meV}}\right)^{-2 / 5}(\beta-1)^{-1 / 5} 45 \mathrm{kpc},
\end{aligned}
$$

With that, we can determine the chemical potential $\mu=\rho^{2} /\left(8 \Lambda^{2} m^{5}\right)$. For $m \sim \mathrm{eV}$ and $\Lambda \sim \mathrm{meV}$, we obtain realistic core sizes, which are of sizes that cover a big part of the halo, as we wanted. For this reason, we choose the fiducial values:

$$
m=0.6 \mathrm{eV}, \quad \Lambda=0.2 \mathrm{meV} .
$$

For these values, we have a cored density profile with a condensate core of radius $158 \mathrm{kpc}$ for $M_{\mathrm{DM}}=10^{12} M_{\odot}$. The condensate does not make the entire halo, but we expect that this condensed core is surrounded by a NFW profile. The central density obtained is smaller than the expected from CDM simulations, which is preferred by observations. In this way the DM superfluid offers a simple resolution to the cuspcore and the "too big to fail" problems. We will see these results in more details in Sect. 4.2.4.

\section{Including baryons}

Now, we derive the condensate profile in the presence of baryons. We expect that there is this extra acceleration due to the interaction to baryons. This comes from the dynamics of the phonon excitation $\phi$ given the Lagrangian (198). We are going to assume a static, spherically symmetric approximation: $\theta=\mu t+\phi(r)$. The equation of motion for the phonon is given by,

$$
\nabla \cdot\left(\frac{(\nabla \phi)^{2}-2 m \hat{\mu}}{\sqrt{(\nabla \phi)^{2}-2 m \hat{\mu}}} \nabla \phi\right)=\alpha \frac{\rho_{\mathrm{b}}}{2 M_{\mathrm{pl}}},
$$

where $\hat{\mu} \equiv \mu-m \Phi$. If we ignore the homogeneous curls term, in the limit where $(\nabla \phi)^{2} \gg 2 m \hat{\mu}$ the solution is

$$
|(\nabla \phi)|(\nabla \phi) \simeq \alpha M_{\mathrm{pl}} \mathbf{a}_{\mathrm{b}},
$$


where $\mathbf{a}_{\mathrm{b}}$ is the Newtonian acceleration due to baryons only. Then acceleration mediated by $\phi$ is,

$$
\mathbf{a}_{\phi}=\alpha \frac{\Lambda}{M_{\mathrm{pl}}} \Longrightarrow a_{\phi}=\sqrt{\frac{\alpha^{3} \Lambda^{2}}{M_{\mathrm{pl}}} a_{\mathrm{b}}}=\sqrt{a_{0} a_{\mathrm{b}}}
$$

for $a_{0}=\alpha^{3} \Lambda^{2} / M_{\mathrm{pl}}$, which is exactly the acceleration expected in the deep MOND regime, as shown in Sect. 2.2.4. In the regime $(\nabla \phi)^{2} \ll 2 m \hat{\mu}$, we recover the Newtonian acceleration given by the baryons. So, in this model, the total acceleration is given by $\mathbf{a}_{\mathrm{b}}, \mathbf{a}_{\phi}$, and also $\mathbf{a}_{\mathrm{DM}}$ the Newtonian acceleration from the DM halo itself (obtained in the previous section), since we have DM in this model (different than MOND).

\section{Halo profile algorithm}

Having developed the theory of the superfluid DM above, now we want to evaluate the density profile of the DM halo and the rotation curves, and compare it with the data to make a first proof of concept of the model. To evaluate the rotation curve, we need to determine the circular velocity with respect to the radius.

As discussed in our model the galaxy contains a superfluid core in the central region of the galaxy surrounded by a NFW profile envelope. So to calculate these quantities for the galaxy we first need to evaluate them inside the superfluid core, and then at $R=R_{\mathrm{NFW}}$ match the density and the pressure obtained for the superfluid $\rho_{\mathrm{SF}}$ and $P_{\mathrm{SF}}$, to the ones given by the full NFW profile.

For that, we need to evaluate these quantities in the superfluid phase. To obtain the halo density profile, we need to determine the total mass of the halo $M(r)$. The rotation curve is the circular velocity with respect to the radius, given by $a=v_{\text {circ }}^{2}(r) / r$ where $a=\partial \Phi / \partial r$. So we need to determine the gravitational potential $\Phi$ to calculate the rotation curve and, also to determine $M(r)$. The Poisson equation in the superfluid region is given by

$$
\nabla^{2} \Phi=4 \pi G\left(\rho_{\mathrm{SF}}+\rho_{\mathrm{b}}\right)
$$

The baryon density is given by the observations, while the superfluid density we can obtain from our theory by differentiating our Lagrangian (198) with respect to $\Phi$,:

$$
\rho_{\mathrm{SF}}=\frac{2 \sqrt{2} m^{5 / 2} \Lambda\left[3(\beta-1) \hat{\mu}+(3-\beta) \frac{(\nabla \phi)^{2}}{2 m}\right]}{3 \sqrt{(\beta-1) \hat{\mu}+\frac{(\nabla \phi)^{2}}{2 m}}},
$$

where we can see that $\rho_{\mathrm{SF}}=\rho_{\mathrm{SF}}(\Phi, \phi)$. So, to solve the Poisson equation, we need the equation for $\phi$, which is given by its equation of motion (205). The system of equations we need to solve is given by (208) and (205), which can be very intricate to solve. One approximation that can be done to simplify this is to assume that baryon distribution is spherically symmetric (which we know it is not true, but used as a simplification). With that, the system can be solved numerically. This is done in Berezhiani et al. (2018). 
Table 1 Summary of observational consequences of superfluid DM, showing the behaviour of this model in each of the systems listed. Table reproduced with permission from Berezhiani et al. (2018), copyright by IOP/SISSA

\begin{tabular}{|c|c|}
\hline System & Behaviour \\
\hline \multicolumn{2}{|l|}{ Rotating Systems } \\
\hline Solar system & Newtonian \\
\hline Galaxy rotation curve shapes & $\begin{array}{l}\text { MOND (+ small DM component } \\
\text { making HSB curves rise) }\end{array}$ \\
\hline Baryonic Tully-Fisher Relation & $\begin{array}{l}\text { MOND for rotation curves (but } \\
\text { particle DM for lensing) }\end{array}$ \\
\hline Bars and spiral structure in galaxies & MOND \\
\hline \multicolumn{2}{|l|}{ Interacting Galaxies } \\
\hline Dynamical friction & Absent in superfluid core \\
\hline Tidal dwarf galaxies & $\begin{array}{l}\text { Newtonian when outside of } \\
\text { superfluid core }\end{array}$ \\
\hline \multicolumn{2}{|l|}{ Spheroidal systems } \\
\hline Star clusters & $\begin{array}{l}\text { MOND with EFE inside galaxy host } \\
\text { core-Newton outside of core }\end{array}$ \\
\hline Dwarf Spheroidals & $\begin{array}{l}\text { MOND with EFE inside galaxy host } \\
\text { core-MOND+DM outside of core }\end{array}$ \\
\hline Clusters of Galaxies & $\begin{array}{l}\text { Mostly particle DM (for both } \\
\text { dynamics and lensing) }\end{array}$ \\
\hline Ultra-diffuse galaxies & $\begin{array}{l}\text { MOND without EFE outside of } \\
\text { cluster core }\end{array}$ \\
\hline Galaxy-galaxy lensing & $\begin{array}{l}\text { Driven by DM envelope } \Longrightarrow \text { not } \\
\text { MOND }\end{array}$ \\
\hline Gravitational wave observations & As in General Relativity \\
\hline
\end{tabular}

After having this, this solution needs to be matched to the NFW profile that describes the outskirts of the halo. With that, it is possible to evaluate the density profile and the rotation curves of galaxies.

\subsubsection{Observational consequences}

In this section, we will describe the main observational consequences of the superfluid DM. A summary of all the effects already worked out can be seen in Table 1. This table compiles a list of the behaviour that this model has in different systems. We describe in this section, some of those results, but point to Berezhiani et al. (2018) for a detailed explanation of each of those cases.

In Table 1, EFE stands for external field effect, and it reveals an interesting characteristic of the superfluid DM model. This effect is an example from kinetic screening in scalar field theories, where in the presence of gradient interactions the scalar acceleration, given by the non-linearities in the scalar field gradient, can suppress scalar field effects in gravity, making the system behave as standard gravity theory. This effect was an essential aspect of MOND, but in the DM superfluid model this effect 
is present in the superfluid cores, coming from the phonon non-linearities. With this, we can see that for satellite galaxies that are inside the superfluid core should follow a MOND-like dynamics, while more distant satellites are outside the core, and therefore should follow Newtonian dynamics. For more details, see Berezhiani et al. (2018).

Since it is going to be used a lot in this section, we remind Landau's conditions for superfluidity is that the fluid velocity $\left(v_{\mathrm{s}}\right)$ is smaller than the superfluid sound speed $c_{\mathrm{S}}, v_{\mathrm{S}}<c_{\mathrm{S}}$.

\section{- Galaxy rotation curves:}

Berezhiani et al. (2018) numerically calculated the rotation curves of IC 2574, a low surface brightness galaxy, and UGC 2953, a high surface brightness galaxy, using the method developed above, as a proof of concept of the galactic dynamics that the DM superfluid is able to reproduce. Since for the theoretical predictions, a spherical baryonic distribution was assumed to simplify the calculations, and since this is far from the actual distribution of baryons, in these calculations a hybrid method, mixing the results calculated with the spherical distribution, was implemented. In this, the acceleration is corrected for the actual distribution leading to

$$
\mathbf{a}_{\text {hybrid }}=\mathbf{a}_{\mathrm{b}, \text { real }}+\mathbf{a}_{\mathrm{DM}}+\mathbf{a}_{\text {phonon }},
$$

where $\mathbf{a}_{b \text {,real }}$ is the acceleration computed from Poisson's equation for a nonspherical baryon distributions; $\mathbf{a}_{\mathrm{DM}}$ the Newtonian acceleration from the DM halo

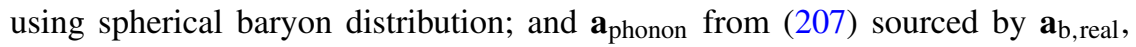
but with Newtonian potential from the spherical case. Although calculated in the hybrid method, $\mathbf{a}_{\text {phonon }} \sim \sqrt{a_{0} a_{\mathrm{N}, \mathrm{b}}}$ as expected in MOND regime.

The fiducial parameters used for this numerical calculation were $m=1 \mathrm{eV}$, $(\sigma / m)=0.01 \mathrm{~cm}^{2} / \mathrm{g}$, which are optimal for having a superfluid core that encompasses the baryonic disk of the galaxy, while still within the bounds to agree with cluster observations; $\Lambda m^{3}=0.05 \mathrm{meV} \times \mathrm{eV}^{3}$; and $\alpha=5.7$. The rotation curves can be seen in Fig. 14.

1. LSB galaxy As pointed out before since these type of galaxies are DM dominated, the rotation curves from LSB are expected to have a slow raise before reaching the plateau region. As we can see in the left panel of Fig. 14, our model reproduces the observed rotation curve for IC 2574, represented by the orange points, very precisely for the parameters chosen.

The size of the superfluid core obtained for this galaxy is $R_{\mathrm{SF}} \sim 40 \mathrm{kpc}$, which here is represented by the NFW radius where the profile is matched with a NFW profile and has a close value to $R_{T}$. Relative to $R_{200} \sim 57 \mathrm{kpc}$ for this galaxy, the superfluid core is relatively large encompassing $58 \%$ of the total DM mass of the halo.

2. HSB galaxy: The rotation curve features of HSB galaxies are known to be hard to be reproduced. We saw that MOND empirical theory is successful in reproducing those features. It is interesting to see if the superfluid DM model is also able to reproduce it. The rotation curve for UGC 2953 is shown in the right panel of Fig. 14, using the same conventions as for the LSB. The radius 
obtained for the superfluid core in this case is $R_{\mathrm{SF}} \sim 79 \mathrm{kpc}$, which is small in comparison to $R_{200} \sim 245 \mathrm{kpc}$. Only $24 \%$ of the total mass of DM is in the superfluid core. The difference from the LSB results is the red curve, where the total DM mass is set by the $\Lambda \mathrm{CDM}$ abundance matching value of $M=65 M_{\odot}$. For the red curve, we get a bigger superfluid radius, $R_{\mathrm{SF}}=93 \mathrm{kpc}$, which is still significantly smaller than $R_{200}=446 \mathrm{kpc}$. The rotation curves seem to fit the data well, showing a smaller value but still compatible with observations for the velocity in the point where the curve turns to flat. Also, the superfluid DM show a slight rise in the end of the rotation curve, which is compatible to the data but not existent in MOND.

In general, it seems that the superfluid model reproduces the rotation curves of LSB and HSB galaxies. Also, the BRTF relation is satisfied, as expected. Of course, this calculation shows a proof of concept and the rotation curves of many more galaxies with different characteristics need to be fitted, also to help determine the parameters of the theory, which were chosen here. However, it is expected that the behaviour of the rotation curve is similar to the fits shown above for different types of galaxies inside the superfluid core, only changing the size of the core depending on the galaxy.

- Dynamical friction: From the very definition of superfluidity, flow without friction, we can expect that in these models in the inner regions of galaxies, where superfluidity emerges upon condensation, dynamical friction to be absent. This might lead to interesting astrophysical consequences and help understand some puzzles with CDM (Hui et al. 2017; Ostriker 1999), while testing the DM superfluid model.

One example of an observation that can be explained by this characteristic of superfluids is the velocity of galactic bars in spiral galaxies, which are expected to have been slowed down by dynamical friction, but are measured to be nearly constant which is consistent with no dynamical friction.

Another interesting puzzle directly linked with dynamical friction is the Fornax globular clusters, as we already mentioned above. In the presence of a superfluid in the halo, given the absence of dynamical friction, these globular cluster should not necessary have merged with Fornax. The effect expected for the case of a superfluid is more pronounced than in the FDM model, for example. This shows that these type of system can offer an opportunity to test these ULDM model.

However, to use these observations to test these models we need to really understand what is the behaviour of dynamical friction in the DM halo. For that, a microscopical description of a superfluid theory with dissipation is necessary, and that is what is shown next.

The simple picture that in a superfluid there is no friction is a simplification. A superfluid can suffer dissipation, when its internal degrees of freedom, are excited out of the condensate, resulting in a mixture of superfluid and normal particles, or even the complete depletion of the condensate. In those cases, then the system exhibits friction. To fully understand how friction and dissipation in the superfluid takes place, one needs to work out the superfluid theories presented in Sect. 3 in the presence of dissipation. To describe dissipation, one needs to study the motion of 
an impurity, a particle moving in the superfluid represented by a real scalar field $\chi$. In general lines, dissipation is described in the following way: if an object passes through this superfluid, this is called an impurity. When this happens, two things can happen: (1) if the object is moving through the condensate with a velocity bigger than the superfluids sound speed, $v>c_{s}$, dissipation of the superfluid takes place, given that the moving object transfers energy to the internal degrees of freedom of the superfluid. At low energies, the only accessible degree of freedom is the phonon, so the passage of such an impurity excites phonons out of the superfluid and the radiation of phonons occurs. The rate of phonon emission,describes the dissipation of the superfluid. The If the impurity passes through the condensate with subsonic speed, then there is no dissipation and the particle travels without friction.

This was discussed in detail in Berezhiani et al. (2019). The regime of validity of such a theory in the presence of dissipation is discussed in Berezhiani (2020), and it is an extension to the discussion presented in Sect. 3, where we assumed the limit without dissipation.

The theory with dissipation was worked out for the simplest superfluid example, the interacting BEC described by the microscopic Lagrangian of a self-interacting complex field (45), with the presence of an impurity,

$$
\mathcal{L}=-\left|\partial_{\mu} \Psi\right|^{2}-m^{2}|\Psi|^{2}-\frac{g}{2}|\Psi|^{4}-\frac{1}{2}(\partial \chi)^{2}-\frac{1}{2} M^{2} \chi^{2}-\frac{1}{2} g_{\text {int }} \chi^{2}|\Psi|^{2} .
$$

We are going to work with the Lagrangian which actually describes the SIFDM model, but that is the simplest model to understand superfluidity. We are interested, as before, in the non-relativistic case and low-energy regimes. To study the dissipation of phonons, we perturb this Lagrangian and work with the linear theory. The process that we want to study is the dissipation of the superfluid radiating phonons caused by the motion of the impurity, with $\Phi$, the Newtonian potential behaving as the mediator of this process. This can be described by the process (at first approximation) $\chi \rightarrow \chi+\pi$, and the rate of this process can be computed.

As discussed in Berezhiani (2020), it is important to reach the correct result to consider the higher order derivatives of the phonon effective action, like we did in (45) that gives rise to the higher order $k^{4}$ term in the dispersion relation, together with the higher order terms involving the impurity field. With that it is possible to calculate the energy dissipation,

$$
|\dot{E}|=\int \omega_{k} \mathrm{~d} \Gamma \propto \frac{n g_{\text {int }}^{2} k_{*}^{4}}{m^{3} M^{2} V},
$$

where $\mathrm{d} \Gamma=\left(q / E_{\chi}^{\mathrm{in}}\right)|\mathcal{A}|^{2} \delta^{(4)}\left(p_{\chi}^{\text {in }}-p_{\chi}^{\mathrm{f}}-p_{\pi}^{\mathrm{f}}\right)\left(\mathrm{d}^{3} p_{\chi}^{\mathrm{f}} / E_{\chi}^{\mathrm{f}}\right)\left(\mathrm{d}^{3} p_{\pi}^{\mathrm{f}} / E_{\pi}^{\mathrm{f}}\right)$ is the rate of the process described above, with 'in' indicating the initial values before the collision and ' $f$ ' indicating final values. The initial momentum of the impurity is $p_{\chi}^{\text {in }}=\left(M+M V^{2} / 2, M \mathbf{V}\right)$ and with $k_{*}$ given by $k_{*}^{2} / 2 M+\omega_{k_{*}}=k_{*} V$. With that, 
we can determine the friction force in the system:

$$
|F|=\frac{|\dot{E}|}{V}=\frac{m n g_{\text {int }}^{2}}{M^{2}} \frac{\left(V^{2}-c_{\mathrm{s}}^{2}\right)^{2}}{V^{2}} .
$$

This shows that the friction force is not discontinuous, given having friction or no friction in the case of having a superfluid or not, as suggested by Landau, but actually it varies monotonically with the velocity. In the limit where $V$ is equal to the sound speed, then the friction force vanishes, as expected for a superfluid.

If we include gravity in this system, we have an extra term coming from the coupling to gravity which modifies the dispersion relation for the superfluid as shown in Sects. 3.3 and 4.1.4

$$
\omega_{k}^{2}=-m_{\mathrm{g}}^{2}+c_{k}^{2} k^{2}+\frac{1}{4 m^{2}} k^{4},
$$

where there is an additional tachyonic mass term from the gravitational contribution, given by $m_{\mathrm{g}}^{2} \equiv 4 \pi G \rho_{0}$. The presence of this term modifies the Jeans scale and Jeans instability occurs when $k>k_{J}=2 m^{2} c_{\mathrm{s}}^{2}\left(-1+\sqrt{1+\left(m_{\mathrm{g}}^{2} / m^{2} c_{\mathrm{s}}^{4}\right)}\right)$. In this case, as shown in Berezhiani et al. (2019), the force evolves monotonically with the velocity. However, it never reaches zero friction for subsonic velocities, when there is the superfluid, because of the Jeans instability. This shows that the dynamical friction in a superfluid is more complex than the simple dichotomy of absence or presence of friction if there is or not a superfluid. This is an active field of research and might lead to interesting observational consequences for the DM superfluid and SIFDM, which is the model worked out here.

- Galaxy clusters: In a simple way, following the analyzes in Sect. 4.2.1, clusters have large dispersion velocities, and at large distances, of order of $R_{200}, \Upsilon$ is going to be small and thermal equilibrium cannot be achieved. The DM in clusters is in the normal phase. However, as we saw for galaxies, in the central regions the density increases and thermal equilibrium may be achieved. In clusters, only a very small amount can be in the superfluid state, since observations exclude that clusters are largely in the superfluid regime. We can then see the bounds in our mass to have a small amount of superfluid component in clusters that is not in tension with data. We assume that $R_{T} / R_{200} \lesssim 0.1$, which gives, using the relations from Sects 4.2.1 and 2.1:

$$
R_{T} \lesssim 200\left(\frac{M}{10^{15} M_{\odot}}\right)^{1 / 3} \mathrm{kpc}
$$

We can now repeat the analysis of thermal equilibrium done in Sect. 4.2.1. However, for such a small $R_{T}$ in comparison to the cluster size, we use the full NFW profile for the halo. This yields a constraint in the mass of the DM particles:

$$
m \gtrsim 2.7\left(\frac{\sigma / m}{\mathrm{~cm}^{2} / g}\right)^{1 / 4} \mathrm{eV} .
$$


This combined with the condition from thermalization in galaxies gives the allowed range for the DM mass:

$$
2.7 \mathrm{eV} \lesssim m\left(\frac{\sigma / m}{\mathrm{~cm}^{2} / g}\right)^{-1 / 4} \lesssim 4.2 \mathrm{eV}
$$

From the tightest constraints from approximately 30 merging systems (Harvey et al. $2015), \sigma / m \lesssim 0.5 \mathrm{~cm}^{2} / \mathrm{g}$. This value is in accordance with the one from Sect. 4.2.1, and from the constrain above it gives a DM mass between $1.5 \mathrm{eV} \lesssim m \lesssim 2.4 \mathrm{eV}$. For DM superfluid in this mass range, we have condensation inside galaxies and the condensation in the interior of cluster happens for very small radius, appearing not to be in conflict with what is expected from observations. This constraint can be made broader by assuming a more realistic and not constant cross section. A quantitative analysis via numerical simulations would be ideal to check this result.

- Galaxy mergers The behaviour of merging galaxies is an interesting question, given the superfluid nature, the absence of friction, proposed for the inner core of galaxies. In the absence of friction, it is expected that the merger would make the galaxies pass through each other without interacting. But the existence of these superfluid phases in these merging systems is going to depend on the comparison between the infall velocity for the merging galaxy and the sound speed of the phonon, given by the Landau criteria.

- $v_{\text {infall }} \gtrsim c_{\mathrm{s}}$ - In this regime, the halos are driven out of equilibrium, so coherence of the condensate is broken and the halo will be in the normal phase. The merging process will proceed as in $\Lambda \mathrm{CDM}$, where mergers are fast due to dynamical friction. Thermal equilibrium and condensation will be achieved in the merged halo after some time.

$-v_{\text {infall }} \lesssim c_{\mathrm{s}}$ - In the case of subsonic velocities, the DM halo is in the superfluid phase, and the superfluid cores will pass through each other with almost no dissipation. In this case, dynamical friction is reduced taking a much longer time to the system to merge, and possible multiple encounters.

In our case, the phonons have sound speed $c_{\mathrm{s}}=\sqrt{2 \mu / m}$, and for the fiducial values adopted (204), $c_{\mathrm{s}} \sim 220 \mathrm{~km} / \mathrm{s}$ for a $10^{12} M_{\odot}$ halo. This needs to be compared with the infall velocities of galaxies of a merging system to see how the merger dynamics proceeds.

\section{- Merging clusters}

The Bullet cluster is a system of two merging cluster that was very well investigate observationally. It represents one of the best evidences of the existence of DM (and against alternatives like MOND). This is seen by a segregation in the position of the mass peak (highest concentration of total matter) given by lensing that probes all the matter content, and the one from X-ray measurements, which measures the baryonic matter. This is consistent with the CDM picture, where the DM in the merging processes due to its negligible interaction passes through almost without interaction, while the baryons are slowed down. This poses a problem for theories that do not have DM, like full MOND.

By construction, and as it was seen above, in this model clusters do not develop 
a condensed core of cluster size and have most of the DM in the normal phase. However, the galaxies inside the clusters have a condensed core, and the cluster can develop smaller sized cores in its inner regions. Therefore, when clusters merge, the presence of a core or not also depends if the merger is subsonic or supersonic, obeying or not Landau's criteria like we saw for the merging galaxies.

The outcome of the merging depends on the infall velocities, which determines if most of the DM is in the superfluid phase or in the normal phase in each of the merging clusters. If the infall velocities are subsonic, the superfluid cores are present, and most of the DM will be in the superfluid phase. Any collision between a cluster where DM behaves like a superfluid will follow without dissipation, with the clusters pass through each other without friction. Now, the DM in the normal phase presents self-interactions. Therefore the collision of two clusters in the normal phase would be slowed down due to these interaction.

In the case of the Bullet cluster (Berezhiani and Khoury 2016), to be consistent with observations, at least the sub-cluster must be in the superfluid phase. As we can see, the sound speed of the phonon for the sub-cluster $\left(M_{\text {sub }} \sim 10^{14} M_{\odot}\right)$ is, for our fiducial values, $c_{\mathrm{s} \text {, sub }} \sim 1400 \mathrm{~km} / \mathrm{s}$, while for the main cluster $\left(M_{\text {main }} \sim 10^{15} M_{\odot}\right)$ is $c_{\mathrm{s} \text {, main }} \sim 3500 \mathrm{~km} / \mathrm{s}$. The relative velocity between the clusters is $\sim 2700 \mathrm{~km} / \mathrm{s}$ (Springel and Farrar 2007; Lage and Farrar 2015). If we take this to be the infall velocity, we can see that the sub-cluster is in the superfluid phase, while the main cluster is in the normal phase. With that, the clusters will merge without dissipation and pass through each without friction, as it is expected from observations.

For the Abell 520 "train wreck" (Mahdavi et al. 2007; Jee et al. 2012; Clowe et al. 2012; Jee et al. 2014), another merging cluster system, the DM superfluid model predicts a subsonic merger, with two peaks representing the superfluid component, compatible with the lensing map, and a peak during the normal component, coming from the X-ray luminosity peak. This shows that the DM superfluid framework can accommodate not only the dynamics on galactic scales, but also explain clusters and its merger events.

\section{- Gravitational lensing}

In the case of the full MOND theory, or its relativistic completion TeVeS, because of the absence of DM to be able to explain the relativistic regime makes necessary the introduction of a complicated non-linear term between the scalar field of the theory and baryons, which should also couple to a time-like vector field to give the correct gravitational potential to be able to explain gravitational lensing.

In the case of the DM superfluid, since the theory has DM, we have the superfluid component described by the phonon scalar field, and we have the normal component which provides the time-like vector field $u^{\mu}$. The gravitational potential is then sourced by both dark matter and baryons, as expected.

As we have that the superfluid core resides in the inner part of the galaxy, surrounded by an NFW envelope, gravitational lensing will come primarily from this NFW outer part.

Recently, the DM superfluid model was studied in the context of strong lensing (Hossenfelder and Mistele 2019).

\section{- Gravitational waves}

In the superfluid DM, different than in MOND, the superfluid core is locates in the 

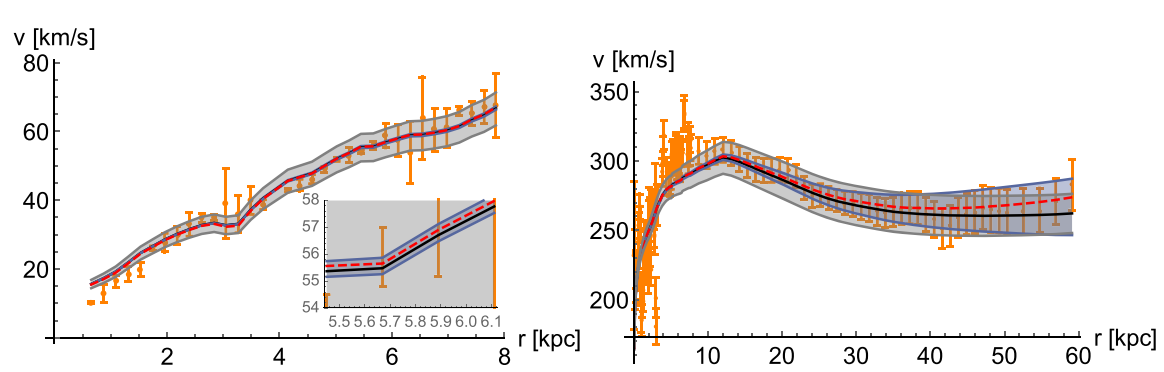

Fig. 14 Left panel: predicted rotation curve for the LSB galaxy IC2574. The orange points are data from Lelli et al. (2016) assuming a distance of $\sim 3 \mathrm{Mpc}$ (Tully et al. 2008), the black and red curves are the predicted curves for $M_{\mathrm{DM}}=20 M_{\mathrm{b}}$ and $50 M_{\mathrm{b}}$. The gray band corresponds to two values of $a_{0} \in(0.6,1.2) \times 10^{-8}$ and the blue band two values of $\Lambda \sim(0.02,0.1) \mathrm{meV}$. Right panel: preducted rotation curve of UGC 2953. The orange points are data from Noordermeer et al. (2007) with all the parameters like in the left panel figure, but the red curve where $M_{\mathrm{DM}}=65 M_{\mathrm{b}}$. Images reproduced with permission from Berezhiani et al. (2018), copyright by IOP/SISSA

inner regions of the halo and the outskirts of the halo have a NFW profile. So the gravitational lensing signal comes from this outer part of the halo and it behaves like in the case of GR+CDM. This means that photons and gravitons propagate at the speed of light travelling along the same geodesics. This is in agreement with the recent constraints from the gravitational waves from neutron stars merger GW170817 (Abbott et al. 2017), which rule out relativistic completions of full MOND (Boran et al. 2018).

The implications for the gravitational waves in the case where the phonon has a non-vanishing sound speed was considered in Cai et al. (2018), together with its observational effects in future GW experiments.

Specifying the microphysics of the DM superfluid particle can also yield other signatures in the produced gravitational waves, like chirality, as done in Alexander et al. (2018).

\subsubsection{Validity of the EFT}

In this section, we are going to scrutinize the validity of the EFT construction used, verifying the regimes where this leading order EFT is valid, and the regimes where the theory obeys the Landau criteria for superfluidity.

\section{Higher-order derivatives}

First, we need to check if in our regime and for the parameters of the model, it is valid to ignore higher order terms in the EFT. As we saw above, the EFT is constructed by including all the terms which are invariant under shift symmetry. We retained only the first order contributions, given that we are working in the low-energy limit. Higher order terms involve more than one derivative per field. Higher order contributions to the quadratic Lagrangian for the phonon (193), can contain terms of the form:

$$
\mathcal{L}_{\text {higher-order }} \supset \Lambda m^{3 / 2} \mu^{\frac{3}{2}-n} \partial^{n} \phi^{n} \sim\left(\Lambda m^{3 / 2} \mu^{3 / 2}\right)^{1-\frac{n}{2}} \partial^{n} \phi_{\mathrm{c}}^{n},
$$


where $\partial \rightarrow \partial_{t}$ or $c_{\mathrm{s}} \nabla$, and $\phi_{\mathrm{c}}=\Lambda^{1 / 2} m^{3.4} \mu^{-1 / 4} \phi$ is the canonical variable. The scale that controls these higher order terms is given by $\Lambda_{\mathrm{s}}=\left(\Lambda m^{3 / 2} \mu^{3 / 2}\right)^{1 / 4}$, which we call the strong coupling scale. So, higher order corrections can be neglected when:

$$
\frac{1}{\Lambda_{\mathrm{s}}} \frac{\partial_{r}^{2} \phi}{\partial \phi} \sim \frac{1}{\Lambda r} \ll 1
$$

This is the general condition for ignoring the higher order corrections in an EFT given by the Lagrangian we described here. Given this, we an easily see that the approximation of ignoring these terms breaks for small sound speeds.

However, specializing to the parameters of the DM superfluid model described here, and using the profile obtained in (202), which determines $\mu$, the strong coupling scale is given by the DM superfluid model:

$$
\Lambda_{\mathrm{s}} \sim \operatorname{meV}\left(\frac{M_{\mathrm{DM}}}{10^{12} M_{\odot}}\right)^{3 / 10}\left(\frac{m}{\mathrm{eV}}\right)^{6 / 5}\left(\frac{\Lambda}{\mathrm{meV}}\right)^{2 / 5}
$$

For the fiducial parameters, $\Lambda_{\mathrm{s}} \sim \mathrm{meV}$. So, higher derivatives are suppressed if $r \gg 0.2 \mathrm{~mm}$, which is clearly satisfied on astrophysical scales.

\section{Criteria for condensate coherence}

An important criteria to verify the validity of the superfluid description we are using is to check if our superfluid obeys the Landau criteria. As we saw in Sect. 3.2, the criteria for the system to transports charge without dissipation, leading to the coherence of the BEC to be maintained, is that the velocity of the superfluid is smaller than the critical velocity:

$$
v_{\mathrm{s}} \ll v_{\mathrm{c}} \sim\left(\frac{\rho}{m^{4}}\right)^{1 / 3}
$$

where the critical velocity must be non-vanishing. This condition is equivalent to the condensation conditions showed in the panel in Sect. 3.1. In the case of the DM superfluid, in Sect. 4.2.1, we already evaluated the conditions for DM to be condensed in the center of galaxies.

We need to estimate $v_{\mathrm{c}}$ locally. For that, we can use the halo mass density $\rho=$ $(2 m)^{3 / 2} m \Lambda \sqrt{|X|} \sim 2 m^{2} \Lambda \sqrt{\kappa}$, where we assumed MOND regime in the last equality and $\kappa=m \hat{\mu}$, which gives us

$$
v_{\mathrm{c}} \sim 0.025\left(\frac{M_{\mathrm{b}}}{10^{11} M_{\odot}}\right)^{1 / 6}\left(\frac{m}{\mathrm{eV}}\right)^{-2 / 3}\left(\frac{\Lambda}{\mathrm{meV}}\right)^{2 / 9}\left(\frac{\mathrm{kpc}}{r}\right)^{1 / 3}
$$

where $M_{b}$ is the baryon's mass. The superfluid velocity is given by $v_{\mathrm{s}}=\partial_{r} \phi / m \sim$ $\sqrt{\kappa} / m$, which yields

$$
v_{\mathrm{s}} \sim\left(\frac{M_{\mathrm{b}}}{10^{11} M_{\odot}}\right)^{1 / 2}\left(\frac{m}{\mathrm{eV}}\right)^{-1}\left(\frac{\Lambda}{\mathrm{meV}}\right)^{-1 / 3}\left(\frac{\mathrm{kpc}}{r}\right) .
$$


With that, using Eq. (221) and assuming spherical symmetry, we an determine thee radius where superfluidity can occur:

$$
r \gg\left(\frac{M_{\mathrm{b}}}{10^{11} M_{\odot}}\right)^{1 / 2}\left(\frac{m}{\mathrm{eV}}\right)^{-1 / 2}\left(\frac{\Lambda}{\mathrm{meV}}\right)^{-5 / 6} \mathrm{kpc}
$$

We can see that this condition is satisfied in the central regions of galaxies, and we have coherence of the condensate and superfluidity in those scales.

\section{Solar system}

We can check this criteria for condensate coherence locally to verify in which regions and regimes we have DM in the superfluid phase. Therefore, we can apply the criteria for condensation developed above and the radius where locally the superfluid can occur to our solar system.

At solar system scales the bounds on deviation from standard Newtonian gravity are very tight, and these measurements do not allow deviations from the Newtonian dynamics. Full MOND is in tension with these bounds. However, the DM superfluid scenario fits well into the Solar system bounds. We can see that using the coherence bound for the condensate (221). For that we need to evaluate $v_{\mathrm{s}}$ and $v_{\mathrm{c}}$ for solar system quantities.

Given the local gradients of the phonon in the vicinity of the $\operatorname{Sun}\left(M_{\mathrm{b}}=1 M \odot\right)$, the superfluid velocity is given by:

$$
v_{\mathrm{s}}^{\odot}=5\left(\frac{m}{\mathrm{eV}}\right)^{-1}\left(\frac{\Lambda}{\mathrm{meV}}\right)^{-1 / 3} \frac{\mathrm{AU}}{r}
$$

where $r$ is the distance to the Sun and AU is the astronomical unit, the average distance between the Earth and the Sun. The critical velocity of the Milky Way galaxy (for $\left.M_{\mathrm{b}}=3 \times 10^{11} M_{\odot}\right)$ evaluated at our solar system $(r \sim 8 \mathrm{kpc})$ is:

$$
v_{\mathrm{c}}^{M W} \sim 0.02\left(\frac{m}{\mathrm{eV}}\right)^{-2 / 3}\left(\frac{\Lambda}{\mathrm{meV}}\right)^{-2 / 9}
$$

We can see that the coherence bound $v_{\mathrm{s}}^{\odot} \ll v_{\mathrm{c}}^{\mathrm{MW}}$ is obeyed for distance much larger than the solar system scales:

$$
r \gg r_{\mathrm{s}}^{\odot}=250\left(\frac{m}{\mathrm{eV}}\right)^{-1 / 3}\left(\frac{\Lambda}{\mathrm{meV}}\right)^{-5 / 9} \mathrm{AU} .
$$

This shows that on solar system scales, the presence of the Sun causes the local phonon gradients to be large, making the local superfluid velocity $v_{s} \sim \phi^{\prime} / m$, to be larger than the local critical velocity for a region around the solar system with a radius $r_{\mathbf{s}}^{\odot}$ from the Sun. Therefore, on distances like the solar system, $r<r_{\mathbf{S}}^{\odot}$, DM is in the normal phase since the condensate loses its coherence, and obeys standard Newtonian gravity. 


\subsubsection{Relativistic completion}

As we saw in Sect. 3.3, the description of a superfluid is given by a weakly selfinteracting field theory with global U(1) symmetry. The symmetry is spontaneously broken by the superfluid ground state of a system at chemical potential $\mu$. In the previous section, where we defined this field theory for superfluids, we added a 2-body self-interaction, $g-3|\Psi|^{4}$. This gives an equation of state $P \propto n^{2}$. As we saw in the previous section, the pressure that describes the interaction in the Madelung equations has the form of the pressure of a barotropic fluid. For a three-body interaction, the equation of state is given by $P \propto n^{3}$. For the DM superfluid, to reproduce MOND, we wanted to have a theory that gave $P \propto n^{3}$. So one might think that the DM superfluid could be described by the microscopic theory of an interacting BEC with three-body interaction. However, we are going to show now that this is in fact not the case since those theories give a Lagrangian with different signs.

\section{Three-body interaction}

Lets consider now like before that the self interacting theory with U(1) symmetry that gives us the superfluid has a three-body interaction, instead of a two-body one. The relativistic action of this theory is given by

$$
\mathcal{L}=-|\partial \Psi|^{2}-m^{2}|\Psi|^{2}-\frac{g_{3}}{3}|\Psi|^{6}
$$

where $g_{3}>0$ for stability. Like before, this theory conserves particle number. Since we are interested in the non-relativistic (NR) theory, replacing $\Psi=\psi e^{i m t}$ and taking the NR limit gives us

$$
\mathcal{L}=\frac{i}{2}\left(\psi \partial_{t} \psi^{*}-\psi^{*} \partial_{t} \psi\right)-\frac{|\nabla \psi|^{2}}{2 m}-\frac{g_{3}}{24 m^{3}}|\psi|^{6} .
$$

With that, we can calculate the equation of motion, which gives us the Schrödinger's equation:

$$
-i \partial_{t} \psi+\frac{\nabla^{2} \psi}{m}-\frac{\lambda}{8 m^{3}}|\psi|^{4} \psi=0
$$

The condensate is described by the background solution, at zero temperature: $\psi_{0}=$ $\sqrt{2 m} v e^{i \mu t}$, where $\mu=\lambda v^{4} / 2 m$. The excitations are given by

$$
\psi=\sqrt{2 m}(v+\rho) e^{i(\mu t+\phi)},
$$

where $\rho$ is the gapless mode and $\phi$ is the Goldstone boson associated with the broken $\mathrm{U}(1)$. At low energies, we substitute this into (229) and integrate out the gapless mode:

$$
\mathcal{L}=\frac{4}{3} m\left(\mu+\dot{\phi}-\frac{(\nabla \phi)^{2}}{2 m}\right)\left[\frac{2 m}{\lambda}\left(\mu+\dot{\phi}-\frac{(\nabla \phi)^{2}}{2 m}\right)\right]^{1 / 2}=\frac{4}{3}\left(\frac{2}{\lambda}\right)^{1 / 2} m^{3 / 2} X \sqrt{X},
$$


which is the action to leading order in the derivative expansion, with $X=\mu+\dot{\phi}-$ $(\nabla \phi)^{2} / 2 m$. This is very promising since the theory with a three-body interaction gives a low-energy Lagrangian with the same exponent as the one we need for MOND and for the effective Lagrangian $P(X)$ for the EFT of superfluids. However, it has the opposite sign, given that $g_{3}>0$ ! As we saw before, the limit where $g_{3}<0$ is unstable, and we cannot have condensation on all scales, superfluidity and MOND.

\section{Phenomenological relativistic Lagrangian}

So, the expected description as a theory with three-body processes is does not work for the DM superfluid model, where we want to recover MOND behavior in galaxies. It was phenomenologically proposed in Berezhiani and Khoury (2015) a relativistic Lagrangian that is able to reproduce our expected Lagrangian (191) in the non-relativistic regime, which is given by

$$
\mathcal{L}=-\frac{1}{2}\left(\left|\partial_{\mu} \Psi\right|+m^{2}|\Psi|^{2}\right)-\frac{\Lambda^{4}}{6\left(\Lambda_{\mathrm{c}}^{2}+|\Psi|^{2}\right)^{6}}\left(\left|\partial_{\mu} \Psi\right|+m^{2}|\Psi|^{2}\right)^{3}
$$

The scale $\Lambda_{\mathrm{c}}$ was introduced in order for the theory to admit $\Psi=0$ vacuum. It is easy to see that this action reduces, in the non-relativistic limit and when $\Lambda_{\mathrm{c}} \ll|\Psi|^{2}$, this action gives (191). The condition for MOND, given by $\Lambda_{\mathrm{c}}$ can be rewritten as $|X| \gtrsim \Lambda_{\mathrm{c}}^{4} /\left(2 m \Lambda^{2}\right)$, which corresponds to

$$
a_{\phi} \gtrsim \frac{\Lambda_{\mathrm{c}}}{\alpha^{2} \Lambda} a_{0},
$$

where $a_{\phi}$ is the acceleration from the phonon that can be obtained from the action and given by $a_{\phi}=\alpha\left(\Lambda / M_{\mathrm{pl}}\right) \phi^{\prime}$. According to observations, the deep MOND regime is very accurate for $\sim a_{0} / 10$, which poses a bound for $\Lambda_{\mathrm{c}}$.

This theory presented here is a phenomenological relativistic version of the DM superfluid. However, it would be interesting to have a relativistic complete microscopic theory for the superfluids.

\subsubsection{Cosmology}

After working out the galactic behavior of the DM superfluid model, we need to understand what happens on cosmological scales in this model. In this section, we show how DM superfluid behaves cosmologically. Since we do not have a proper relativistic theory that we can use to describe cosmology, we make some estimates to understand the behaviour of DM on cosmological scales in this theory.

The first question we would like to answer is if DM is in the superfluid or normal phase cosmologically. We saw in Sect. 4.2.1 that the critical temperature of the DM superfluid is given by Eq. (185), and $T / T_{\mathrm{c}}$ today is around $10^{-2}$ for massive galaxies $\left(M \sim 10^{12} M_{\odot}\right)$. Cosmologically, the temperature is much colder. We can estimate given that ultra-light candidates for DM, like the DM superfluid, are non-thermal relics. They can be generated, for example, through a vacuum displacement mechanism (see 
below for a definition of this mechanism) like the axion. So, the particles are created when $H_{i} \sim m$, which corresponds to a temperature for the photon-baryon plasma:

$$
T_{i}^{\mathrm{b}} \sim \sqrt{m M_{\mathrm{pl}}} \underset{m \sim \mathrm{eV}}{\longrightarrow} 50 \mathrm{TeV}
$$

which is around the weak scale!

With that, we can rewrite the condition for thermalization (90), given that the velocity and density redshifts as $v \propto a^{-1}$ and $\rho \propto a^{-3}$. At matter-radiation equality, we can write this condition as

$$
m \sim \rho_{\mathrm{eq}}^{1 / 4} \ll\left(\frac{\rho_{\mathrm{eq}}}{v_{e q}^{3}}\right)^{1 / 4}
$$

where $\rho_{\mathrm{eq}} \sim 0.4 \mathrm{eV}^{4}$ and using that $v_{\mathrm{eq}}=v_{i} a_{i} / a_{\mathrm{eq}} \sim \mathrm{eV} / \sqrt{m M_{\mathrm{pl}}}$ is much smaller than one, while $v_{i} \sim 1$ since it was created deep into the radiation era. Since $T / T_{\mathrm{c}}=$ $\left(v / v_{\mathrm{c}}\right)^{2}$, we have that

$$
\left(\frac{T}{T_{\mathrm{c}}}\right)_{\text {cosmo }} \sim v_{\mathrm{eq}}\left(\frac{m}{\mathrm{eV}}\right)^{8 / 3} \sim 10^{-28}\left(\frac{m}{\mathrm{eV}}\right)^{5 / 3}
$$

So, cosmologically, all the DM is in the superfluid state. Once this DM is formed, if condenses and behaves as a superfluid. However, one question than comes to mind: as we saw previously, in the DM superfluid model in the superfluid phase the dynamics is give by a MONDian dynamics, instead of Newtonian. Then the question is: does DM behaves differently than Newtonian on cosmological scales, which can be a problem to reproduce some known results in cosmology? We can see that this is not the case.

The cosmological temperatures are many orders of magnitude different than the temperatures on galaxies. For the EFT built for the superfluid to be valid on such different scales, the parameters of the EFT $\Lambda$ and $\alpha$ need to evolve with the temperature. This dependence is estimated in Berezhiani and Khoury (2015) by making some phenomenological statements for the theory to match both regimes. A consequence of the introduction of this variation is that the critical acceleration, given by $a_{0}$ in galaxies, is now temperature dependent. Therefore, on cosmological scales the critical acceleration of the theory has a much smaller value than the one from galaxies: $a_{0}^{\text {cosmo }} \ll 10^{-4} a_{0}$. This has an important consequence: although the DM superfluid is condensed on cosmological scales, the gravity is highly Newtonian on those scales.

This shows to us that a very compelling feature of this model: at the same time it describes the small-scale behavior, given by a MOND-like dynamics, it also recovers the large-scale successes of CDM. It also recover the expected CDM behaviour in clusters, and also in the vicinity of star like in the solar system, where the DM is in the normal phase behaving like particle DM.

To close the DM superfluid section, we can summarize the global behaviour that this model has on all the scales. This together with Table 1 describes the phenomenology of the DM superfluid class. 


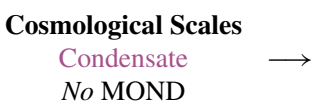

No MOND

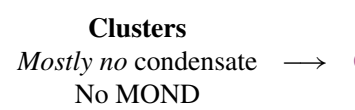

Galaxies

Condensate $\longrightarrow$ MOND
Solar System

No condensate

No MOND

\subsection{Simulating ULDM models}

We described above the main characteristics of the ULDM models. We showed how we expect the small-scale structures to be suppressed in this model by computing quantities in the linear limit, and showed how this model presents a core solution for a simplified model of the halo. However, to study the formation of structures at different scales, and the formation of galaxies which are highly non-linear processes, one needs to resort to simulations. Cosmological simulations have been one of the biggest tools for the understanding of the non-linear formation and evolution of structures and galaxies in the past few years, modelling diverse scales and physical process that are present in those processes. Therefore, to better understand how different the structures are going to be in the ULDM models, together with modelling some structures that are exclusively present in these constructions like the presence of cores, interference and vortices, we need to resort to cosmological simulations (for a summary of the current FDM simulation, see Zhang et al. 2019; Li et al. 2019).

The traditional simulation methods present in the literature to study the formation of structures, like N-body simulations or hydrodynamical simulations, cannot be readily applied to the case of ULDM since they do not take into account the wave nature of these models. As we saw above, it is this wave nature that can lead to important observational consequences present in these models of DM.

There are two approaches to simulate the ULDM models. One solves the Schrödinger-Poisson system, composed by the Gross-Pitaevskii equation for a given ULDM model coupled to the Poisson equation; the other is given by solving the hydrodynamical equivalent of the GP equation, the Madelung equations. Each of those approaches have advantages and disadvantages, so they can be considered complimentary.

\begin{tabular}{|l|l|}
\hline Schrödinger-Poisson & Hydrodynamical-Madelung equations \\
$i \dot{\psi}=-\frac{1}{2 m} \nabla^{2} \psi+m \Phi \psi+\frac{g}{8 m^{2}}|\psi|^{2} \psi+\frac{g_{3}}{12 m^{3}}$ & $\frac{\partial \rho}{\partial t}+\nabla \cdot(\rho \mathbf{v})=0$ \\
$|\psi|^{4} \psi+\cdots$ & $\frac{\partial \mathbf{v}}{\partial t}+\rho(\mathbf{v} \cdot \nabla) \mathbf{v}=-\frac{1}{m} \nabla\left(P_{\mathrm{QP}}+\Phi+P_{\text {int }}\right)$. \\
$\nabla^{2} \Phi=4 \pi G m\left(|\psi|^{2}-|\bar{\psi}|^{2}\right)$ & \\
\hline
\end{tabular}

In the absence of interaction, we have the Fuzzy DM model, and in the presence of interaction, the terms marked in blue, we have the SIFDM model. The interactions can be a two-body or three-body interactions, like shown respectively in the last two terms of the GP equation above, or even higher order body collisions represented in the ellipsis, if this is allowed in the system. These describe fluids with different equation of state. In the Madelung equations, the interactions are represented by the interaction pressure term $P_{\text {int }}$, which has the form of a polytropic fluid. This polytropic fluid can describe different fluids with different equations of state, that arise depending on the 

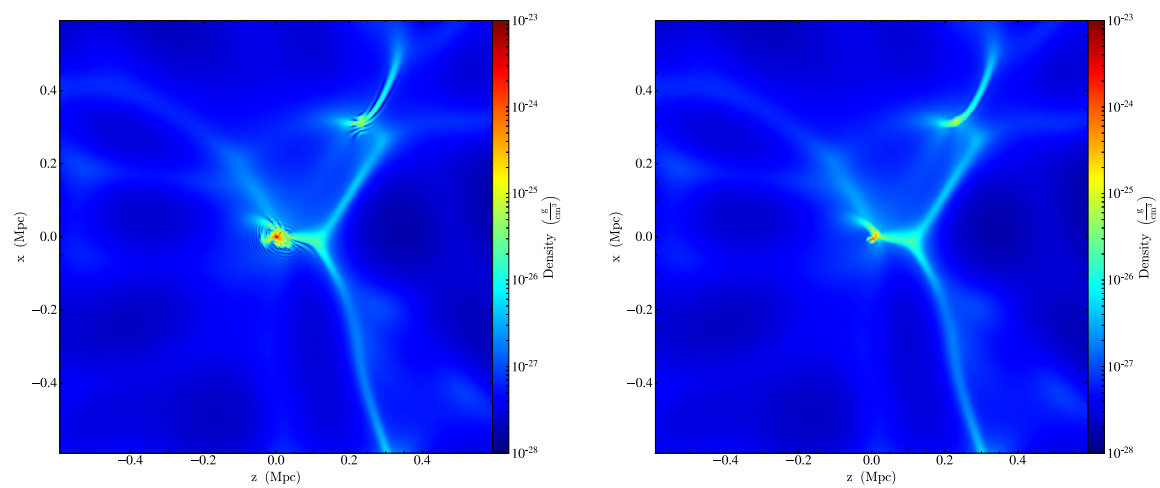

Fig. 15 Wave simulation (left panel), where the SP system is solved, and a fluid simulation (right panel), where the Madelung equations are solved, of the FDM model with mass $m=10^{-23} \mathrm{eV}$. This represents a slice at redshift $z=5$ of the density, given by the color code, and it shows how the wave simulation can resolve the small-scales by showing the interference patterns, that are coarse grained by the fluid simulations. Images reproduced with permission from Li et al. (2019), copyright by AAS

type of interaction. So one can simulate either one of those models using these system of equations ${ }^{43}$.

For the simulation that solve the Schrödinger-Poisson equations, also called wave simulations, there are many groups that are attempting to solve this system using different methods (Schive et al. 2014a; Schwabe et al. 2016; Mocz et al. 2017, 2018; Edwards et al. 2018; Garny et al. 2020). In general, this approach is very good to describe the small-scales, being able to resolve the small structures and taking into account the wave nature of the condensate, as we can see in the left panel of Fig. 15. With that, this approach predicts the correct and expected structures on small-scales. Being able to resolve the smaller scales, this simulation can resolve and show the presence of cores in the halos, the granular interference structure in the condensate or the presence of vortices. However, this approach is very demanding numerically, since it requires a more finely resolution to resolve scales of the order of the de Broglie wavelength. This makes these simulations to be much smaller in size than the fluid ones, not describing cosmological scales or being able to span many decades in redshift.

The simulations that solve the Madelung equations, the fluid simulations, have the advantage of being able to use the already written and well explored hydrodynamical codes available in the literature. They can be implemented by adapting those known codes to the case of FDM or SIFDM. The difference from a normal fluid simulation is the presence of the quantum pressure term, $\nabla P_{\mathrm{QP}}=-n \nabla Q$, where $Q=\left(\hbar^{2} / 2 m\right)\left(\nabla^{2} \sqrt{\rho}\right) / \sqrt{\rho}$. This term is singular when the density is zero, and the quantum pressure is not well defined in this regime. This restriction translate into those simulation not being able to resolve the smallest scales, coarse graining through the

\footnotetext{
43 Theoretically, one could also simulate a gravitationally bounded DM superfluid model by evaluating its the equation of motion in the NR regime, obtaining a equation analogous to the GP equation, but with a more complicated form. There is not, to our knowledge any group that is performing simulations of those models at the moment. We might expect that the more complicated form of the equations might render a more computationally expensive simulation. For this reason we stick for now to the simulations of the other classes of models.
} 
granular structure or any other substructure expected in these models. This leads to fluid simulations predicting a more pronounced gravitational collapse leading to an enhancement bigger than expected in the power spectrum at small-scales ( $\mathrm{Li}$ et al. 2019).

The advantage of the fluid approach, though, is not only being implemented using the already mature hydrodynamical codes, but also being able to run much larger simulations than in the wave case, since it is less computationally expensive. With this method cosmological size simulations are possible. Many groups have been working on simulating the FDM using the fluid approach, with some variations in the implementations of the codes and the solvers (Veltmaat and Niemeyer 2016; Mocz and Succi 2015; Nori and Baldi 2018; Zhang et al. 2018b).

Many research groups are attempting to perform those simulations so we can better understand the behaviour of the FDM model and reveal possible smoking gun signatures of this model. Those simulations are crucial so we can understand and better search for these signatures on observations. For this it would be interesting to have the small and large-scales of the simulations resolved. Since the fluid simulations are good to describe the large-scales and the wave simulations the small-scales, some groups are exploring the possibility of having hybrid simulations where both methods are considered for the scales they work better (Li et al. 2019). Veltmaat et al. (2020) consider another hybrid method where N-body simulations are used to simulate the cosmic web, while the wave simulation is implemented in the inner halo.

Another simulation that is also hybrid is the AxioNyx simulation (Schwabe et al. 2020). This simulation actually mixes different dark matter models, having a Schrödinger-Poisson solver built on a cosmological N-body simulation of CDM and baryons to simulate self-gravitating mixed fuzzy and CDM. This allows to include in this simulation baryonic effects and some astrophysical processes. With this simulation one can study spherical collapse and core formation in this mixed DM context. This mixed DM nature of the simulation can be seen in a suppression in the CDM collapse due to the FDM fraction, while CDM delays the FDM collapse shrinking the Jeans scale. It was also found that both FDM and CDM evolution respond to the same gravitational potential, although in the center of the overdensities, where solitons form depending on the granule mass which is determined by the FDM fraction, a large fraction of FDM particles of about $10 \%$ of the total DM is present.

More work in the presence of interactions, describing the SIFDM, would also be welcomed, since this would reveal more about the superfluid nature of this DM scenario and the possible consequences of having a superfluid core in the inner regions of galaxies.

Simulations of ULDM models are a fast moving and essential field to study these models, and the current advances are very exciting, which makes us look forward to new results in the near future.

We are focusing in this review on dark matter, in the late time non-relativistic limit

\section{SIFDM/superfluid}

There are a smaller number of simulations for the case of the self-interacting ULDM. It is important to also consider systems where self-interaction is present, on top of gravitational interaction, since these systems contain a very rich phenomenology. 

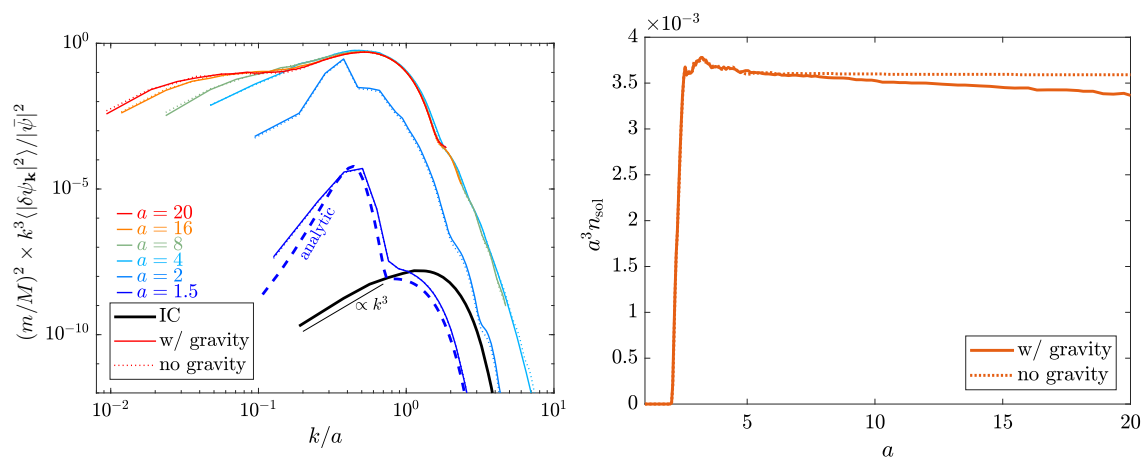

Fig. 16 Left panel: power spectrum of $\Psi$, with adiabatic vacuum fluctuations initial condition, for the case with and without gravity. Growth of perturbations occur first through self-interaction instability, backreacting in the condensate, followed by soliton formation. Right panel: comoving number density of solitons in simulations with and without gravity. The fact that when gravity is included effects like mergers occur makes the number of solitons to be smaller in the case gravity is included. Images reproduced with permission from Amin and Mocz (2019), copyright by APS

Depending on their sign these models present different clustering scales, soliton formation, which in this case can be driven by the self-interaction instead of gravity alone, and its rates, times and sizes; and effects like dynamical friction work differently.

Amin and Mocz (2019) investigated the system of a non-relativistic scalar field in the presence of two-body self-interaction in an expanding universe. The main goal of the authors is to study the formation, clustering and collision of solitons when their formation is controlled by attractive self-interactions, on top of the gravitational part. They work in the regime where the scales of the problem present the following hierarchy: $m \ll M \ll m_{p l}$, where $m$ is the mass of the ULDM particle, $M$ controls the interaction scale, and the reduced Planck mass determines the strength of gravity. In this work, they solve numerically the Schrödinger-Poisson system in the presence of interaction in $3+1$ dimensions in the presence of expansion, with cosmological initial conditions. They compare the result of this simulations with analytical results calculated for the soliton formation time and length scales, the soliton distribution and two-point function of the clustering of the solitons, showing good agreement between both. The problem then has two instability scales from the self-interaction and from gravity, as shown separately in Sect. 4.1.4. We show in left panel of Fig. 16 the power spectrum of the scalar field. The formation of solitons controlled by the interactions is faster than under gravity alone, as seen in the right panel of Fig. 16. In the presence of those two components, the solitons scatter, merge and form binary systems, the last only present in the presence of gravity. This shows that the system with interactions presents significant different phenomenology than in the case of FDM. Therefore, more efforts to simulate these interacting BECs is necessary, specially in the case of repulsive interaction.

Another interesting discussion present in this paper is the connections to the equivalent relativistic system: a relativistic non-linear Klein-Gordon equation. In the relativistic system, the equivalent to the soliton is the oscillon. In this review we are interested in DM, so discussing simulations of relativistic system is not in the scope of the paper. But it is worth mentioning that there is a large body of literature in this 
topic (see, e.g., Kolb and Tkachev 1994; Amin et al. 2012; Lozanov and Amin 2019). They describe the mainly the early universe evolution, but are directly applicable in the context of ULDM.

\section{Two-fluid approach}

As we mentioned in Sect. 3, based on the ideas by Landau, the correct description of a finite temperature superfluid is using the two-fluid model, where there is a normal and a superfluid components. The description used above for the SIFDM and DM superfluid extend the limit of usage of the zero temperature description of the superfluids when describing DM in a halo, which should be treated with a finite temperature approach. An effective extension to include finite-temperatures was attempted for the DM superfluid case. When simulating the superfluid model, one should use the equations for a superfluid in the two-fluid formalism, where the system is described as a mixture of a superfluid component (represented with subscript ' $s$ ') and a normal fluid (represented with subscript ' $n$ '). The superfluid is the component that flows without friction while the normal fluid is the only of the two that transports entropy and thermal energy.

This is what it was done in Hartman et al. (2019). They want to study structure formation in the a model where DM forms a superfluid. For this reason, they solve the hydrodynamical equations that describe this superfluid. The finite-temperature hydrodynamical equations for the simplest superfluid, the weakly interacting Bose gas in a trapped potential, are given by Taylor and Griffin (2005); Chapman et al. (2014)

$$
\begin{aligned}
& \frac{\partial \rho}{\partial t}+\nabla \cdot \mathbf{j}=0, \quad \frac{\partial S}{\partial t}+\nabla \cdot\left(S \mathbf{u}_{n}\right)=0, \quad \frac{\partial \mathbf{u}_{s}}{\partial t}+\nabla\left(\mu+\mathbf{u}_{s}^{2} / 2\right)=-\nabla \Phi \\
& \frac{\partial \mathbf{j}}{\partial t}+\nabla P+\rho_{S}\left(\mathbf{u}_{s} \cdot \nabla\right) \mathbf{u}_{s}+\rho_{n}\left(\mathbf{u}_{n} \cdot \nabla\right) \mathbf{u}_{n}+\mathbf{u}_{s}\left[\nabla \cdot\left(\rho_{s} \mathbf{u}_{s}\right)\right]+\mathbf{u}_{n}\left[\nabla \cdot\left(\rho_{n} \mathbf{u}_{n}\right)\right]=-\rho \nabla \Phi \\
& \frac{\partial E}{\partial t}+\nabla \cdot\left[\left(U+\frac{1}{2} \rho_{n} u_{n}^{2}+P\right) \mathbf{u}_{n}+\frac{1}{2} \rho_{s} u_{s}^{2} \mathbf{u}_{s}+\mu\left(\mathbf{u}_{s}-\mathbf{u}_{n}\right)\right] \\
& \quad=-\mathbf{j} \cdot \nabla \Phi
\end{aligned}
$$

where $\rho=\rho_{s}+\rho_{n}$ is the fluid mass density, $S$ the entropy density, $\mathbf{u}$ is the velocity, $\mathbf{j}=$ $\rho_{n} \mathbf{u}_{n}+\rho_{s} \mathbf{u}_{s}$ is the momentum density, $E$ is the energy density E, $\Phi$ the gravitational potential, following the Poisson equation and $\mu=\left[P+U-S T-\left(\mathbf{u}_{s}-\mathbf{u}_{n}\right)^{2} / 2\right] / \rho$ is the chemical potential ( $P$ is the pressure, $U$ the internal energy density and $T$ the temperature).

In this set of equations, the authors set the trapping potential to be the gravitational potential and these equation can be re-written in an expanding background by transforming to super-comoving coordinates and using $\mathbf{v}=\mathbf{u}-H \mathbf{r}$. In this way, the model describes the finite-temperature hydrodynamical equations for the SIFDM model. The authors test the two-body and three-body interactions for comparison.

One challenge of this approach is that the hydrodynamical equations do not include the Landau criteria in them. To include this explicitly one needs to add dissipative terms. Since this is not completely worked out analytically, to do that one has to make 
some assumptions and assume a form for these terms. A very big step in including dissipation in this theory was done by Berezhiani et al. (2019); Berezhiani (2020) and shown above in the "dynamical friction" subsection of the DM superfluid. This is a work in progress. Until this is worked out in detail, and avoiding adding unknown dissipative terms, the authors chose to do this numerically imposing Landau's condition at every position in the simulation.

They numerically integrate the hydrodynamical equations from redshift $z=100$ until today. The authors found from the simulation that, in SIFDM model, the growth of structure proceeds less efficiently than in CDM, as expected for the ULDM models, although more efficiently than expected, with the suppression more pronounced on small-scales and at high temperatures. They also study the role of the interaction strength and of the equation of state.

This numerical simulation presents some limitations giving some of the assumptions and limitations inherited from the hydrodynamical formalism. For example, in this approach we cannot see the complete dissipation of the superfluid. Also, correctly adding the dissipation physics would be a big improvement in this description. However, this simulation represents a very important step towards simulating more realistic superfluids, which already showed to lead to interesting observations consequences.

\subsection{ULDM as dark energy}

Ultra-light fields can also behave as dark energy (DE), depending on their mass and on the different theory they are applied. We can see this in two different cases where the ultra-light field can be used to explain the acceleration of the universe.

\subsubsection{Fuzzy DM}

In the case of fuzzy DM, where we have a (non-interacting) ultra-light scalar field in FRW universe, the field can behave as dark matter, early dark energy or dark energy depending on the mass of this field. The behaviour of the field depends on how its mass is related to the Hubble parameter. At early times, the ultra-light field has $m \ll H$. In this regime, the field is almost frozen and behaves as DE with $w \approx-1$. As $m \sim 3 H$, the field starts to coherently oscillate around the potential minimum and to behave as DM, where the equations of state averaged over the oscillations approaches zero. Depending on the mass, the oscillating DM phase can happen at different times of the evolution of the universe.

If the oscillating phase happens after radiation-matter domination, the ultralight field behaves like DM. From Hložek et al. (2015), we see this happens for $m \gtrsim 10^{-27} \mathrm{eV}$. This bound comes from observations from the CMB and LSS galaxy clustering, and in this regime the ultra-light field can oscillates before the present day and the field redshifts as DM. More generally, for $m \lesssim 10^{-32} \mathrm{eV}$ we can still have the ultra-light field behaving as DM, since the field starts oscillating before the present time, but in this case the ultra-light field can only make a fraction of the DM.

For $m \sim 10^{-33} \mathrm{eV} \approx H_{0}$, the field behaves like a quintessence field, if in the presence of a potential, and it can be the responsible for the late time acceleration. 
Since the field is virtually frozen until the present time, or slowly-rolling the potential, with almost constant density, and it behaves very closely to a cosmological constant. With observations from CMB, we can see that in this case the ultra-light fields have a maximum bound on the energy density compatible with the expected amount of DE in the universe. For masses around $10^{-32} \mathrm{eV} \lesssim m \lesssim 10^{-27} \mathrm{eV}$, the ultra-light field behaves like DE earlier than what it is expected, and can be thought as an early DE component.

\subsubsection{Superfluid DM—unified superfluid dark sector}

There is another way to explain the late time acceleration using these ultra-light fields, where the acceleration is not given by this behaving like a quintessence field. This can be done in the context of the DM superfluid, where the dark energy behaviour is yet another manifestation of the same superfluid that emerges at cosmological scales at late times, as presented in Ferreira et al. (2019); Ferreira (2021).

In the previous case and in the case of quintessence, a ultra-light field is a component with a very small mass $m \approx 3 H_{0}$, that dominates around the present times and drives the acceleration. Differently, in the framework we present here, the dark sector is composed only by DM described by a superfluid, without DE. The late acceleration emerges from the dynamics of this superfluid, and we have a unified model for the behaviour of DM and DE. We can see how this arises from the model described below.

BECs and superfluids in the laboratory are usually made of atomic species. After the discovery of BEC and superfluidity, one big evolution in the study of these systems was to study mixtures of condensates where atoms that compose the fluid that condenses might be at different atomic configurations. This allowed researchers to explore the richness of the internal structure of the atoms that compose the superfluid, describes a more realistic system, where atomic transitions are allowed to happen, and also the different dynamics that appears in systems where more than one species of condensate and superfluid is present.

In this entire section, we are using the same formalism as the one defined for the DM superfluid (and the EFT of superfluids from Sect. 3.4. In the model we present here, we assume that the superfluid is composed by two different species, which can be represented by the ground $\left(\Psi_{1}\right)$ and excited $\left(\Psi_{2}\right)$ state of the dark atom that composes the superfluid. These species interact through a Josephson interaction (Josephson 1962; Tommasini et al. 2003) ${ }^{44}$, which is a contact interaction between the components of the superfluid that has the simple form $\mathcal{L}_{\text {int }} \propto-\left(\Psi_{1}^{*} \Psi_{2}+\Psi_{1} \Psi_{2}^{*}\right) /\left|\Psi_{1}\right|\left|\Psi_{2}\right|$. So the Lagrangian of this theory is the Lagrangian for the EFT of superfluids, given by a noncanonical kinetic term, plus the term coming from the interaction. This interaction

\footnotetext{
44 The Josephson interaction or Rabi coupling (Josephson 1962; Mahan 2000) is very common in many systems in condensed matter systems. It is a contact interaction that represents a long-range phase coherence between components, leading to conversion between the different species. This is used in many systems leading to the very well known Josephson effect, but it is also present in other effects studied theoretically and experimentally (Usui and Takeuchi 2015; Cappellaro et al. 2017; Bornheimer et al. 2017; Fernandes and Chubukov 2017; Nicol and Carbotte 2005; Ballagh et al. 1997; Zibold et al. 2010).
} 

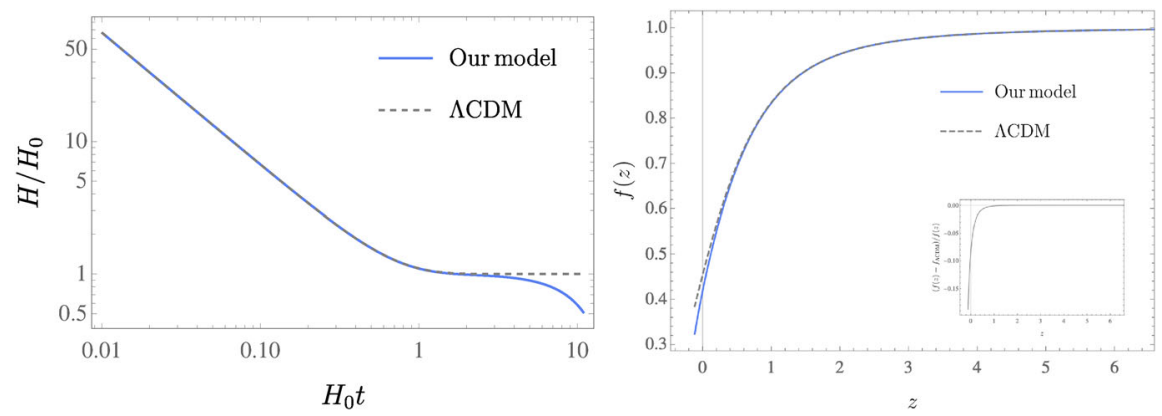

Fig. 17 Left panel: evolution of the Hubble parameter for the unified DM-DE model with superfluids in comparison to $\Lambda \mathrm{CDM}$. We can see that both model describe the same cosmology given by a period of deceleration where the universe is matter dominated, followed by an acceleration period, around present times. The unified model deviates from the $\Lambda \mathrm{CDM}$ evolution close to present and for future times, where the action of the oscillatory potential is more pronounced. Right panel: evolution of the growth factor in the unified model in comparison to $\Lambda \mathrm{CDM}$, and percent difference, showing that those differ for present times given the potential that describes the accelerated expansion period

leads to an oscillatory potential for the low-energy Lagrangian of the phonons,

$$
\begin{aligned}
& \mathcal{L}=P_{1}\left(X_{1}\right)+P_{2}\left(X_{2}\right)-(1-2 \Phi) V\left(\theta_{1}, \theta_{2}\right), \quad \text { with } \\
& V\left(\theta_{1}, \theta_{2}\right)=M^{4}\left[1+\cos \left(\theta_{2}-\theta_{1}+\Delta E t\right)\right],
\end{aligned}
$$

From the form of the interaction term, the oscillatory potential for the phonons is given by a cosine potential, where $M$ is the explicit symmetry breaking scale coming from the interaction Lagrangian that breaks softly the shift symmetry of the phonon action, and that has to be of the order of $M^{4}=2 M_{\mathrm{pl}}^{2} H_{0}^{2} \approx \mathrm{meV}$, to drive the late time acceleration. The parameter $\Delta E$ is the energy gap between the two species, between the ground and first excited state of the component of the superfluid.

Considering the approximation $\Delta E \ll m_{i}$, we can see that the superfluid has two distinguished behaviours: one degree of freedom that behaves like dust, described only by the non-canonical kinetic term that behaves like DM in the non-relativistic regime as the DM superfluid model, and one that evolves under the influence of the potential, like what is expected from a field that behaves like DE. The cosine potential is similar to the pNGB models of DE (Frieman et al. 1995; Kaloper and Sorbo 2006), and it is a special potential for explaining DE since it only softly breaks the shiftsymmetry, and the flatness of this potential is still approximately protected against radiative corrections, which is one big problem in quintessence models of DE. The late time acceleration behaviour from this DM superfluid can be seen in the evolution of the Hubble parameter in the NR limit,

$$
2 \dot{H}+3 H^{2}=V\left(\theta_{1}, \theta_{2}\right) / M_{\mathrm{pl}}^{2} \text {. }
$$

From the left panel of Fig. 17 we can see that we have a decelerated evolution, following the behaviour of DM, followed by a period of accelerated expansion at present time. Therefore, this model behaves like what is expected by DE, even without the presence 
of a specific component responsible for the acceleration, and being a model of DM alone. At future times, this models deviates a lot from the predictions of $\Lambda \mathrm{CDM}$, as the cosine potential becomes important.

Although the evolution in this model is very close to $\Lambda \mathrm{CDM}$, this model presents distinct predictions. This can be seen by computing the perturbations in this model. One of those predictions is the growth factor, that in this model deviates from the $\Lambda \mathrm{CDM}$ one by around $10 \%$ at present times, as presented in right panel of Fig. 17. Future galaxy surveys might be able to test this deviation.

\section{Cosmological and astrophysical constraints, and new windows of observation}

Now that we have described our ULDM classes and showed the consequences that these models might have in cosmology and astrophysics, in this section, we are going to show some of the constraints obtained for the parameters of these theories when the different phenomenology of these models is tested with data.

For most part of this section, the constraints are going to be for the FDM model. This model has been much more explored in the literature than the others, not only because it has been introduced first, but also since it has only one parameters $m$ (we are assuming in general that the ULDM is the total mass of the universe, unless stated otherwise). However, we are also going to show some constraints obtained for the SIFDM and the DM superfluid models. In each part it is stated for which model the constraints are obtained.

We summarize most of these constraints on the mass FDM in Fig. 18. As we can see in this figure, this set of (current) constraint, if they hold, strongly suggests that an FDM with mass of order of $10^{-22} \mathrm{eV}$, which was proposed as the ideal mass that introduces interesting new phenomena on small-scales and that addresses the controversies that appear in those scales, is strongly challenged. For the heavier masses that seem to be allowed now, the phenomenology of the FDM is closer to the one from CDM.

In the plot we presented only some of the constraints present in the literature. Other bounds obtained from other observables testing different astrophysical consequences of the ULDM models is presented below, together with a description of the bounds of the figure ${ }^{45}$.

We just want to emphasize that this bounds are for the FDM model only, and the SIFDM and the DM superfluid have other sweet spots for the mass of their ultra-light particle. These two models currently are weakly constrained with not a lot of work done in the literature to constraint the parameter of these models. For the DM superfluid, as we saw in Sect. 4.2, the mass is constrained to be $1.5 \mathrm{eV}<\mathrm{m}<2.4 \mathrm{eV}$ coming from the thermalization condition in the halo with cross-sections that are allowed by measurements from galaxy cluster mergers.

\footnotetext{
45 The bounds presented here assume that almost all the DM is composed by FDM. If one wants to see an equivalent figure that takes into account the fraction of the FDM, see Fig. 1 from Grin et al. (2019). This reference also presents a very good review of the gravitational probes of FDM.
} 


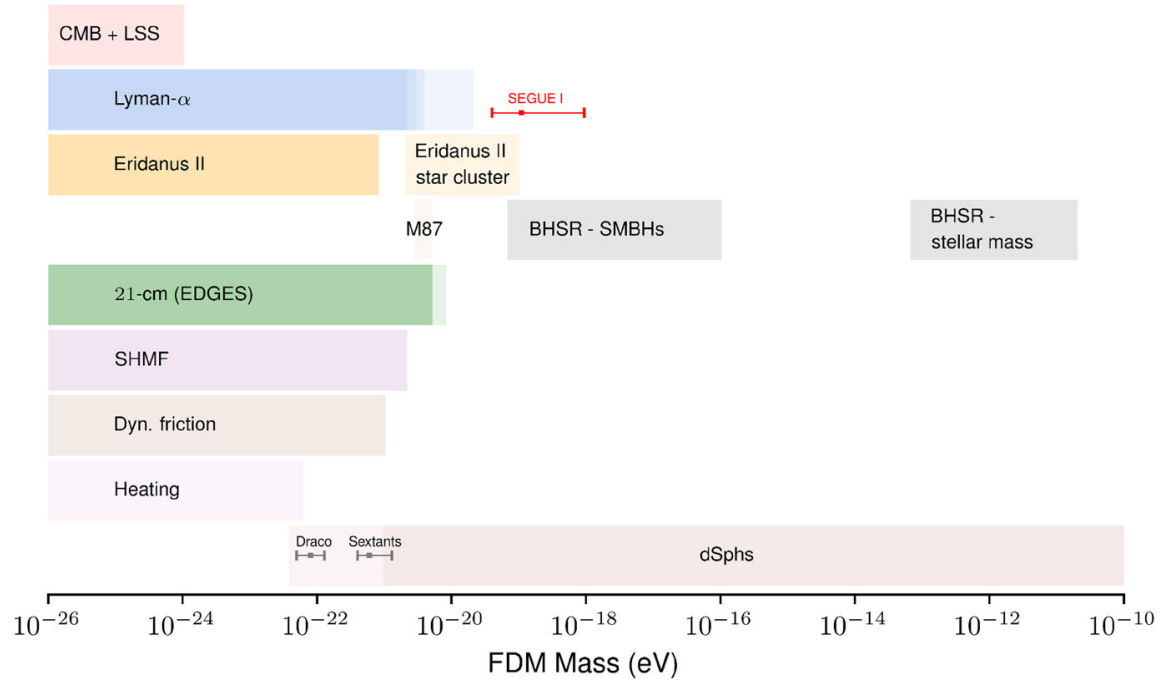

Fig. 18 Summary of most of the constraints on the mass of the FDM particle discussed in the section (For other versions of this figure and a notebook to generate it check https://github.com/elisaferreira/ figure_mass_FDM.). These bounds assume that FDM makes most of the DM in the universe. In this figure, the shaded regions represent the excluded regions. The CMB and LSS bounds come from Hložek et al. (2015, 2018) using Planck (2015) TT CMB auto-power and the WiggleZ galaxy-galaxy auto-power spectrum. The Lyman- $\alpha$ constrains correspond different analysis made in the literature coming, from the darker to lighter, from Nori et al. (2019); Armengaud et al. (2017); Iršič et al. (2017); Rogers and Peiris (2020), respectively. The Eridanus II constraint are both for its existence and for the survival of its star cluster from Marsh and Niemeyer (2019). The next line presents the constraints from black hole superradiance (BHSR). The first constraint comes from bounds on the spin of the supermassive BH (SMBH) in M87, from the measurements obtained by the Event Horizon Telescope (Davoudiasl and Denton 2019). The second set of bounds comes from Stott and Marsh (2018), which presents the stringiest bounds from BHSR of ultra-light particles from stellar BHs and from SMBHs. The global $21 \mathrm{~cm}$ signal detected by the EDGES team can also be used to put bounds on the mass of FDM as shown in (Lidz and Hui 2018; Schneider 2018). The next row refers to bounds on the FDM imposed by testing the suppression of the sub-halo mass function in comparison with the SHMF from WDM models constrained using strong gravitational lensing of quasars and from fluctuations in stellar streams (Schutz 2020). In Lancaster et al. (2020), they compute the different description that dynamical friction has for the FDM and apply this to the Fornax globular cluster. The next bound comes from another dynamical effect, which is heating of the MW disk, that can be constrained measuring the velocity dispersion of stars in the solar neighbourhood (Church et al. 2019). We also include two constraints in the mass assuming that the measured central density of dSphs, Draco and Sextants should match maximum FDM core size, which should be smaller then the virial radius of these galaxies (Chen et al. 2017). This row also contains the results from the reanalysis of the bounds from dSphs from González-Morales et al. (2017) starting at the lighter region, and Safarzadeh and Spergel (2019) the darker shaded region. In red, it is the constraint in the mass coming from the analysis of the ultra-faint dwarf Segue I from Hayashi et al. (2021)

\subsection{Cosmological constraints: CMB and LSS}

We are first going to talk about the constraints and forecasts that can be obtained on the ULDM parameters using cosmological observations. 


\subsubsection{CMB and LSS}

\section{FDM}

We saw in Sect. 4.1.4 how the angular temperature power spectrum and the matter power spectrum can be affected by the FDM. We show now constraints obtained in this class of models using measurements of the CMB power spectrum and of the matter power spectrum. These constraints are obtained in mainly in two articles. In Hložek et al. (2015), the authors investigated that using a combination of CMB data from the Wilkinson Microwave Anisotropy Probe (WMAP), Planck satellite, and also from ground CMB experiments like the Atacama Cosmology Telescope, and South Pole Telescope, and galaxy clustering data from the WiggleZ. And in Hložek et al. (2018) this analysis was updated, and some additional effects were tested, using a combination of data from Planck (2013) temperature power spectrum and the WiggleZ galaxy-galaxy auto-power. In these references, they investigate the FDM model, where only one particle is responsible for the FDM.

In those two papers, they investigate the effects in the $\mathrm{CMB}$ and in the matter power spectrum of a large range of FDM masses, encompassing masses where the ultra-light particles behave as dark energy. These two papers also investigate effects that could come from specific models inside the FDM, like the constraining the axion isocurvature modes, and the spontaneous symmetry breaking scale, which we are not going to discuss in this review.

In these references, the data from CMB and from LSS were combined to obtain the constraints. The combination of this data is important to make the constraints on the higher mass end of the FDM tighter, and it is driven mainly by the LSS tight constraints for $k \sim 0.1 \mathrm{hpc}^{-1}$.

For the low mass end of the FDM, the combination makes the constraints weaker. This results show that if one wants a DM component that can be responsible for all the DM in the universe, then

$$
m \gtrsim 10^{-24} \mathrm{eV}
$$

If $m \lesssim 10^{-32} \mathrm{eV}$ the ultra-light field can behave as dark energy. This constraints are driven mostly by the expansion of the universe given that a component with this mass modifies the evolution of the universe after matter-radiation equality and can be strongly constrained by CMB (we will discuss FDM as DE in more details in the end of this section). The most up to date constraints are present in Hložek et al. (2018), but we show in Fig. 19 a very explanatory plot from Hložek et al. (2015) where we can see these constrains. The allowed region is the red-shaded region.

The region where $10^{-32} \mathrm{eV}<m<10^{-23} \mathrm{eV}$ is interesting since is allows to constrain the model a lot. This might not be the region where the ultra-light field can have a sizable DM component, but it allows to put percent level constraints the fraction of the FDM, with CMB being the best gravitational probe in this regime. In this region, FDM cannot be more than $5 \%$ of the total DM: $\Omega_{\mathrm{FDM}} /\left(\Omega_{\mathrm{DM}}+\Omega_{\mathrm{FDM}}\right) \leq 0.05$ and $\Omega_{\mathrm{FDM}} h^{2} \leq 0.006$ with $95 \%$ confidence level. 


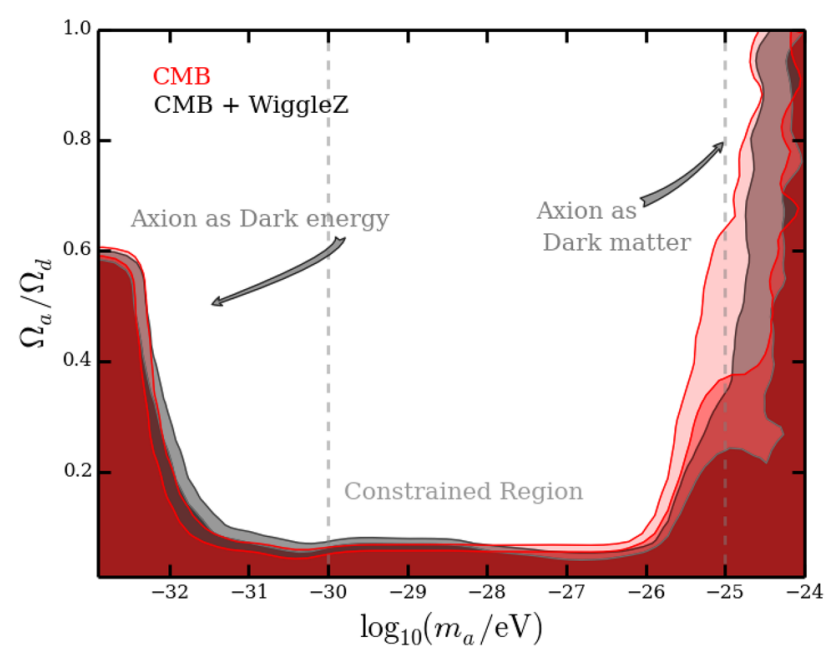

Fig. 19 The $2 \sigma$ and $3 \sigma$ of the mass fraction $\Omega_{\mathrm{ultra}-\text { light }} / \Omega_{\mathrm{d}}$ in function of mass. The regions show the constrained region $\Omega_{\mathrm{ultra}-\text { light }} / \Omega_{\mathrm{d}} \lesssim 0.05$ at $95 \%$, where $\Omega_{\mathrm{d}}$ is the total dark-matter density fraction. CMB-only constraints are the red regions, while grey regions include large-scale structure data. Image reproduced with permission from Hložek et al. (2015), copyright by APS

Future CMB experiments, CMB S4, will be much more sensitive to the energy density of the ultra-light particles (Hložek et al. 2017). They will be capable of probe different imprints that ultra-light particles can leave in the CMB in the range of masses $10^{-32} \mathrm{eV}<m<10^{-23} \mathrm{eV}$. This range is particularly interesting to probe many different aspects of the microphysics of the models that belong to the FDM class, like the axion and ALPs. In the higher mass range of the above interval, this next generation of CMB experiments can provide constraints in the mass that are competitive to smallscale observables like dwarf galaxies abundances and mass-halo profiles.

Optical depth The suppression of the structure formation present in the FDM model leads to a possible smaller amount of galaxies at high-z, changing the reionization history. We saw that the optical depth can be changed by the FDM (164). We can then use the optical depth measured by the CMB, $\tau\left(r_{\text {rec }}\right)$, to constrain the mass of the FDM. This was done in Bozek et al. (2015) where they use the value of the optical depth from Planck + WMAP (Spergel et al. 2015). They found that a mass $m \lesssim 10^{-23} \mathrm{JeV}$ for all the DM to be FDM is excluded, depending on the model chosen which entails details of the reionization and the luminosity function, at more then $3 \sigma$. The standard mass of $10^{-22} \mathrm{eV}$ is challenged by these high-z measurements, with results from this mass being on the edge of the allowed parameter space. This shows that non-linear high-z measurements can also be used to constrain the FDM mass, and other ULDM models. However, these bounds carry a lot of astrophysical uncertainties and so need to be considered carefully.

This study opens up an interesting avenue to constrain the FDM model through the modified reionization using CMB. The epoch of reionization can be better constrained by measuring the kinematic Sunyaev-Zel'dovich (kSZ) effect (Sunyaev and Zeldovich 1980). The amplitude of the kSZ is sensitive to the duration of the reionization, and 
could be used to put bounds in the FDM mass. Experiments like Advanced ACTPol $(\mathrm{AdvACT})$ have an improved measurement of the $\mathrm{kSZ}$ and help constrain the FDM.

\subsubsection{Lyman- $\alpha$}

\section{FDM}

Recent investigation of FDM models in light of Lyman- $\alpha$ forest finds new constraints on FDM mass (Iršič et al. 2017; Armengaud et al. 2017; Kobayashi et al. 2017). It puts a bound in the mass of FDM in the case where more than $30 \%$ of the DM being composed by this scalar field of $m \gtrsim 10^{-21} \mathrm{eV}$. This value is larger than the value necessary for the FDM model to solve the small-scale problems of $\Lambda \mathrm{CDM}$, and puts some tension in the FDM model.

The Lyman- $\alpha$ forest is produced by the absorption of the light from quasar by clouds of neutral hydrogen localized at low-redshifts, in the line of sight between the quasars and us. For this reason, this is an important probe of the matter spectrum on small-scales, on scales of order $0.5 \mathrm{Mpc} / h \lesssim \lambda \lesssim 100 \mathrm{Mpc} / h$.

In this work, data from the XQ-100 survey were used which refers to 100 medium resolution spectra with emission redshift $3.5<z<4.5$. This data is compared against a simulation of the FDM with different masses and abundance today. On non-linear scales, quantum pressure is added. The result is shown in the left panel of Fig. 20. The right panel of this figure we also see the impact of the constraints obtained in cosmology and in the astrophysical implications. In cosmology, the constraints obtained give a bound in the value of the displaced field, assuming that the genesis mechanism for this light field is vacuum displacement. Combining this data with CMB data, they also derive bounds on inflation, more specifically on $r$ the tensor to scalar ratio for an inflationary epoch in the presence of FDM. They also show how this bound impacts the resolution of the small-scale problems presented by FDM. The cyan line indicates the bound where the missing satellites problem is solved by FDM. The constraint is very tight and it shows a tension with the Lyman- $\alpha$ measurements.

A possible caveat from this analysis, and of the other in Iršič et al. (2017) and Armengaud et al. (2017), is that they use hydrodynamical simulations, and they mostly neglect quantum pressure. However, quantum pressure can be very important and play a vital role in structure formation, which is what the analytical behaviour seems to show us (Zhang et al. 2018a). In a follow-up paper (Nori et al. 2019), quantum pressure was included in the simulations and its effect on the LSS evolution is studied. It is found a constraint in the mass of the FDM model of $m \sim 2.1 \times 10^{-21} \mathrm{eV}$, which is compatible with the ones obtained in the analyses that do not include quantum pressure (Iršič et al. 2017; Armengaud et al. 2017; Kobayashi et al. 2017). It is found that quantum pressure does not affect the LSS in these redshifts and scales relevant for Lyman- $\alpha$, not affecting the bound on the mass largely. These simulations also allow the authors to study the properties of the halos formed in this model, showing the differences in their distribution and shape in comparison to CDM.

Recently, Rogers and Peiris (2020) presented a reanalysis of the Lyman- $\alpha$ data. This is based on a method that emulates the power spectrum from a "training" simulation constructed using Bayesian optimization, which then is fed in the MCMC sampling 
of the parameter space. The authors claim that this emulator makes less assumptions than the usual interpolation techniques and for this reason presents a better statistical modelling of the power spectra. With this technique they obtain a even higher bound for the FDM particle: $m>2 \times 10^{-20} \mathrm{eV}$, which disfavours the strongly $10^{-22} \mathrm{eV}$ canonical FDM mass. This reference presents the newest and most complete study of the bound on the FDM model using Lyman- $\alpha$, and it confirms the tension with the value of the mass that is necessary in order for the FDM model to address the small-scale challenges.

However, as it is pointed out in these references, the bounds obtained above depend on how the intergalactic medium (IGM) is modelled. It is expected, for example, that as reionization proceeds in a spatially inhomogeneous manner, fluctuations in the temperature and ionization must be present, and, therefore, the IGM model should consider this effect. As pointed out in Hui et al. (2017) and some of the references above, differences in this modelling like missing the addition of these fluctuations could drastically change these bounds. In the recent analysis by Rogers and Peiris (2020), however, where they marginalize over physically-consistent IIGM models with different temperature and ionization histories it is argued that this can actually tighten the constraint. They also claim that the current data might be only marginally sensitive to these different modellings of the IGM. How to model an the impact of the IGM modelling in the FDM bounds, remains, then, a question that needs further investigation. Therefore, new and independent analysis needs to be done to confirm if the intermediary to small-scales hold more information about these models. Another probe that can help with that is the $21 \mathrm{~cm}$ from neutral hydrogen, since it probes even smaller scales.

Another thing that could change these bounds is the properties of the FDM model. Leong et al. (2019) pointed out that for FDM models with a axion-like cosine potential, different initial conditions can yield different bounds on the FDM mass when using the Lyman- $\alpha$ data. Different than in the standard case considered until now where we considered small angles (field values — see discussion below (93), in the extreme axion misaligned angle is considered, the transfer function presents a bump for small-scales. This affects the Lyman- $\alpha$ flux power spectrum. With this initial conditions, the mass of this FDM scenario necessary to explain the Ly- $\alpha$ data is of order $10^{-22} \mathrm{eV},>10$ times bigger than for the case of standard FDM initial conditions considered above (see Arvanitaki et al. 2020 for other phenomenology of this extreme FDM model). This shows that the bounds also depend on the properties of the FDM model.

\subsection{3 $21 \mathrm{~cm}$ cosmology}

\section{FDM}

As discussed above, the ULDM models give a suppression of the matter power spectrum on small-scales. Those scales can only be marginally probed by the cosmological probes like CMB, LSS, cluster abundance, Ly- $\alpha$ forest. Those measurements can only constraint the structures on scales $k \leq 10 \mathrm{Mpc}^{-1}$, not being able to probe the smaller scales. One new window of observation, the $21 \mathrm{~cm}$ line from neutral hydrogen $(\mathrm{HI})$, promises to allow us to probe the matter power spectrum in much smaller scales, $k \geq 10 \mathrm{Mpc}^{-1}$, on scales even smaller than the Ly- $\alpha$ forest. This is possible since neutral hydrogen is only present in the universe, after reionization, inside dense 

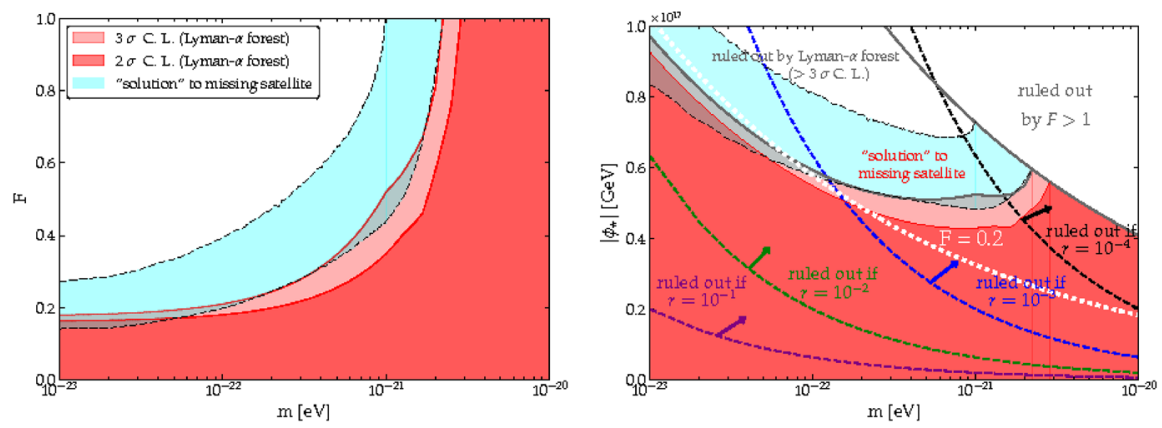

Fig. 20 Left panel: shows the constraint on the mass of the FDM and the fraction of the total DM mass from Ly- $\alpha$ forest measurements. Right panel: constraints in the mass of the FDM and the value of the displaced field. This is combined with cosmological constraints, shown by the dashed lines for different tensor to scalar ratios. The region in parameter space where the missing satellite problem is solved for the FDM model, is shown by the cyan stripe. The white dotted contour represents the line where FDM constitutes only $20 \%$ of the total DM. Image reproduced with permission from Kobayashi et al. (2017), copyright by APS

clouds in damped Ly- $\alpha$ systems, which are small objects $\left(k \sim 10^{2} \mathrm{Mpc}^{-1}\right)$. In this way the $21 \mathrm{~cm}$ HI signal is a biased tracer of the galaxies, and consequently of the underlying matter distribution in such small-scales. Therefore, measuring the global $21 \mathrm{~cm}$ HI signal together with its fluctuations can gives information about the still largely unconstrained small-scale matter power spectrum.

However, at those scales these system are dominated by astrophysical process, making it difficult to disentangle the behaviour of DM from those processes. To obtain cosmological information from these measurements is a challenge. Some recent studies (Muñoz et al. 2020) forecast that the matter power spectrum can be measured from the global $21 \mathrm{~cm}$ HI signal for an experiment with parameters close to EDGES (Bowman et al. 2018), with a precision of $\mathcal{O}(10 \%)$ integrated over the scales $k=(40-80) \mathrm{Mpc}^{-1}$, after imposing priors on the astrophysical effects like star formation rate and feedback amplitude. They also parametrize the effect of foregrounds, like the Galactic foreground, that plagues all $21 \mathrm{~cm}$ experiments and might represent a huge limitations for them if not well mitigated. Detecting the $21 \mathrm{~cm} \mathrm{HI}$ fluctuations is a much harder task. Large interferometer experiments like HERA (Hydrogen Epoch of Reionization Array) (DeBoer et al. 2017), LOFAR (LOw-Frequency ARray) (van Haarlem et al. 2013), LWA (Long Wavelength Array) (Eastwood et al. 2019), and SKA (Square-Kilometer Array) (Koopmans et al. 2015) have the goal of measuring this signal from the epoch of reionization (EoR) and also late times. For a HERA-like experiment it was found that the matter power spectrum can be probed with an accuracy of $\mathcal{O}(10 \%)$ integrated over the scales $k=(40-60) \mathrm{Mpc}^{-1}$ and $k=(60-80) \mathrm{Mpc}^{-1}$. The measurements of the fluctuations probe the evolution of the matter density tomographically, carrying more information about the scale and redshift dependency of the HI signal, bringing more information on the astrophysical processes. This makes the parameters of these astrophysical processes to be better disentangled from the HI signal, allowing this constraint in the power spectrum be less dependent on the astrophysics in these regions. 
Specifically for the case of ULDM models, forecasts using $21 \mathrm{~cm} \mathrm{HI} \mathrm{signal} \mathrm{were}$ made in Lidz and Hui (2018); Nebrin et al. (2019); Shimabukuro et al. (2020), and they specialize in the FDM model. Lidz and Hui (2018) studied how the recent EDGES measurement of the global $21 \mathrm{~cm} \mathrm{HI} \mathrm{signal} \mathrm{(Bowman} \mathrm{et} \mathrm{al.} \mathrm{2018)} \mathrm{can} \mathrm{constraint} \mathrm{the}$ FDM model. The global signal is the average radio signal from $21 \mathrm{~cm}$ redshifted emission from $z \sim 15-20$ in the case of EDGES. This measurement showed an absorption profile that had an amplitude two times bigger than the expected. This higher amplitude indicates that already at redshift $z=20$, there was significant star formation, which leads to a also significant $\operatorname{Ly} \alpha$ background. This fact shows that the smallest structures, and consequently the power spectrum on small-scales, cannot be largely suppressed. This puts constraints in models of DM that have the feature of suppressing the small-scale structures, like the FDM (or any ULDM model). This measurement alone is capable of putting a challenging constraint in the mass of the FDM particle: $m \geq 5 \times 10^{-21}$. A similar analysis is performed in Schneider (2018), where using conservative limits of the stellar to baryon fraction and minimum cooling temperature motivated by hydrodynamical simulation puts a comparable bound in the FDM particle mass: $m \geq 8 \times 10^{-21}$. To obtain this constraint some assumptions on the star formation, and on the halo mass profile had to be made. Given the importance of this result for the FDM models, the bounds obtained from this data should be explored further, as well as the future data from $21 \mathrm{~cm}$ signal.

To better understand how the HI signal is affected by the FDM model, Nebrin et al. (2019) study the impact of FDM models in the $21 \mathrm{~cm}$ HI signal from the cosmic dawn and EoR analytically, together with some forecasts for future experiments. They use an analytic model which take into account the Ly- $\alpha$ coupling, X-ray heating and ionization to study the $21 \mathrm{~cm}$ in a $\Lambda$ FDM cosmology. They find that suppression of structure from FDM models, which makes small sub-halos absent in this model, has the effect of postponing the formation of sources and the reionization of neutral hydrogen. This delay changes the global $21 \mathrm{~cm}$ signal showing a smaller absorption feature than expected from $\Lambda \mathrm{CDM}$. The amount of suppression allowed considering the results from the EDGES experiment puts a lower bound on the mass of the FDM model, with $m \geq 6 \times 10^{-22} \mathrm{eV}$, which might already be considered challenging for the FDM to solve the small-scale problems, but marginally. They also show the potential of a SKA-like and LOFAR experiments to test these models in the future. This shows us that future experiments will be able to confirm the important bound of the FDM model imposed by the EDGES.

Shimabukuro et al. (2020) analyze the impact of measurements of the $21 \mathrm{~cm}$ forest, which alternatively from the tomographic and power spectrum techniques to use 21 $\mathrm{cm}$ HI signal, proposes to use the $21 \mathrm{~cm}$ narrow absorption features from the IGM cause by high-z loud radio sources or collapsed objects, like minihalos. The $21 \mathrm{~cm}$ forest is expected to be measured by SKA. In this reference they show that the impact of this measurement can also constraint the mass of the FDM model, and that this is degenerate with the fraction of FDM that composes the DM.

For post-reionization HI signal from $0<z<3$, that can be measured using the intensity mapping technique, a study of the forecast of the possible constraints in the FDM model was presented in Bauer et al. (2020). This analysis shows how this signal can be used as a powerful probe of the halo formation since the halo abundance is 
changed if a fraction of the DM is given by the FDM. They forecast the constraints in the mass of the FDM for a SKA1MID-like IM experiment. They find that the fiducial value adopted for the FDM $\left(m=10^{-22} \mathrm{eV}\right)$ can be constrained at the $10 \%$ level when the $21 \mathrm{~cm}$ data are combined with $\mathrm{CMB}$ data from the Simons Observatory.

The possibility of $21 \mathrm{~cm}$ HI signal to constrain alternative models to CDM, like WDM, for example, was studied in many references (Barkana et al. 2001; Yoshida et al. 2003; Safarzadeh et al. 2018; Lopez-Honorez et al. 2019; Leo et al. 2020). They show how the signal changes for different DM models and also show how measurements like the one from EDGES can put constraints in the mass of WDM.

The study of the capabilities of $21 \mathrm{~cm}$ experiments to give us cosmological information is an active field of study and the references above are just some examples of those efforts. These studies give us hope that, in the near future, this new window of observation will allow us to probe the still unconstrained small scales, helping elucidate the nature of the DM component.

\subsection{Astrophysical constraints and new windows of observation}

We presented above some cosmological observations that help to constraint the ULDM models. In the past few years, there has been a huge advance in the observations of the small-scales, with new windows of observations being opened that can help determine the nature of DM. We present in this section, the constraints on the ULDM parameters coming from astrophysical observations and present some of these new windows of observations that are still being tested and being developed, but that promise to help testing the ULDM models.

\subsubsection{Local Milky Way observables and stellar streams}

Our galaxy, the Milky Way (MW), is our closest source of information about DM and it is a very good laboratory for studying its behaviour on small-scales. Here we present some observations from the MW that promise to help us test different models of DM. We are in a very special era for observations of the MW and Local group with data coming from many current and future experiments like Gaia (Gaia Collaboration 2016), Large Synoptic Survey Telescope (LSST) (Ivezić 2019), Prime Focus Spectrograph (PFS) (Tamura et al. 2016), WFIRST (Akeson 2019), among others. Using the incredible new data from these observations promises to be revolutionary in the studies of the MW, and hopefully for helping discover the nature of DM.

\section{MW's gravitational potential}

Very quickly we would like to point out an observable that can help us test DM. Knowing the shape, mass and distribution of the halo in the MW can gives us clues on the DM model since different DM models predict distinct shapes for the halos. To understand the halo we need information not only in the inner regions of the halo, but on many different scales up to the virial radius. The measurement of the position and velocity of satellite galaxies and globular cluster can give information for the dynamics in a good range of distance from the center of the MW. The distribution of 
satellite galaxies is already used to put constraints on the mass of the ULDM models (and other models of DM), as we saw above.

Experiments like Gaia can give us very accurate data for scales much smaller than the virial radius. PFS galaxy archaeology survey will also measure stars in the galactic disk, complementing complementing Gaia's survey. LSST is expected to provide information from stellar tracers on scales close to $R_{\text {vir }}$, being able to measure many new satellites that are fainter and more distant than the known today, extending the determined halo mass function by three orders of magnitude.

This is linked to the study of streams discussed above, since streams given their long range in the halo, can give us information on the gravitational potential of the halo for of even larger scales.

\section{Dwarf galaxies}

Dwarf galaxies are good laboratories to study ULDM models. Those small galaxies are DM dominated and allow us to study the behaviour of DM in an environment with small influence from baryonic effects. They can be used to probe the three classes of effects we saw in Sect. 4.1.6: the suppression of the power spectrum, effects coming from the core structure inside the galaxy, and dynamical effects. We can use many different types of dwarf galaxies for that from dwarf spheroidals (dSphs) to ultra-faint dwarfs (UFD).

The suppression of the power spectrum present in the FDM model, leads to a suppression in the low-mass halos. So the FDM model predicts halos with a minimum mass. Nadler et al. (2019) used the minimum mass of detected halos from the observed population of satellites in the MW, and found that in order for it to be within the bound (182), the mass of the FDM needs to be $m>2.9 \times 10^{-21} \mathrm{eV}$, setting a lower bound from on the mass caused by the linear suppression.

The effects from the presence of the core in the interior of galaxies can also be probed by dSphs. As discussed in Sect. 4.1.4, classes of models like the FDM present a limit for the size of the cores that they can form which leads to an upper bound in the central density of these cores. We can use measurement the central density and half mass radius of dwarf galaxies to compare with those bounds and constrain the mass of the FDM.

As we already discussed, McConnachie (2012) measured half-light radii inferred from the densities of 36 Local Group dwarf spheroidals, and when compared with the bound on the half mass radius predicted for the FDM (180), obtained a mass around $m \sim 10^{-22} \mathrm{eV}$ so these are compatible. The density of 8 dwarf spheroidals has been measured in Chen et al. (2017). Comparing these central densities measured with the bound (181) it was shown that for the central density from FDM to match the measured ones from the dSphs Draco and Sextants, the mass of the FDM needs to be $m=8_{-3}^{+5} \times 10^{-23} \mathrm{eV}$ for Draco and $m=6_{-2}^{+7} \times 10^{-22} \mathrm{eV}$ for Sextans. For those masses, the FDM leads to a cored distribution at the center of the galaxies, alleviating the cusp-core problem. This shows that dSphs can be used to put bounds on the mass of FDM.

Nevertheless, new studies have been reviewing these bound. They challenge many aspects of this result including the analysis made, presenting some reanalysis, and the assumption of sphericity of the halo of these galaxies. 
In González-Morales et al. (2017), it was suggested this analysis might be giving biased values for the FDM mass. The reason for that is because there is a degeneracy between the mass density profile and the anisotropy of the velocity dispersion. When using Jeans analysis to obtain the halo parameters, like the FDM mass, from dSph galaxies that we do not know the density profile, leads to a biased determination if this mass. Therefore, in this paper they use mock catalogues of dSphs hosted in a FDM halo and they conclude that the analysis should be fitting the luminosity-averaged velocity dispersion of the subcomponents. Using this technique for Fornax and Sculptor, they obtain a bound in the mass of the FDM with $97.5 \%$ confidence of $m<0.4 \times 10^{-22} \mathrm{eV}$, which goes in a different direction than the other constraints.

Safarzadeh and Spergel (2019), using the half-mass radius and the slope the mass profile of Fornax and Sculptor dwarf spheroidal galaxies, obtained a different bound for the FDM mass $m \lesssim 10^{-21} \mathrm{eV}$, to have the expected density profile and halo mass for those dSphs from observations like dynamical friction.

Kendall and Easther (2020) pointed out yet another possible limitation of the above analysis. They show that the presence of cores with flat density profile in the center of the NFW-halos can actually make the density of large ULDM halos larger than the CDM ones, making the cusp-core problem worse. This happens because solitons obey the inverse mass-radius scaling law, with mass depending on the total mass of the halo. They perform an analysis that takes into account semi-analytically the variability of the core-halo relation showing that this might make this discrepancy less strong for larger halos. However, this shows that many aspects that are crucial for properly describing these systems, like fluctuations and baryonic effects, are not present in the semi-analytic model.

Another study also challenges this result based on the fact that the DM halo in dSphs might not be spherical (Hayashi and Obata 2020). This analysis produces less stringent bounds due to uncertainties in the non-sphericity but brings an important characteristic that should be considered about the DM halo of dSphs. There are also several works in the literature (e.g. (Read et al. 2019; Safarzadeh and Spergel 2019; Hayashi et al. 2020)) that suggest that luminous dSphs might be affected by baryonic effects which makes the analysis of the dark matter profile in those system to be more complicated.

Another class of dwarf galaxies that can be used to probe ULDM model are the ultra-faint dwarf galaxies. UFDs are considered the ideal system to study dark matter since they present an even larger mass to light ratio than other dwarf galaxies, containing less stellar mass than the luminous dSphs, and being less affected by baryonic effects Lazar et al. (2020). There are only a few studies of the FDM model using UFDs, but they already show the importance of these system to probe the properties of DM on small-scales, in particular of the FDM model. Calabrese and Spergel (2016), using the half-light masses of Draco II and Triangulum II estimated $m_{\text {FDM }} \sim 3.7-5.6 \times 10^{-22} \mathrm{eV}$. Doing a similar analysis using Milky Way UFDs, Safarzadeh and Spergel (2019) obtained a bound in the FDM mass, $m_{\text {FDM }}>10^{-21} \mathrm{eV}$. We can also cite studies using Eridanus II, which we will mention in a separate section.

Although much less explored, the UFDs put the strongest constraints to date on the FDM mass, as shown in Hayashi et al. (2021). In this work, as more data from stellar kinematics of UFDs become available, it was performed a full Jeans analysis 
of 18 UFDs, where a simulation driven FDM core profile (obtained in Schive et al. $2014 \mathrm{a}, \mathrm{b}$ ) surrounded by an NFW profile is fitted to the data. The authors found that the FDM mass obtained for all UFDs prefer a particle mass heavier than $10^{-21} \mathrm{eV}$. The strongest constraint comes from Segue I, $m_{\mathrm{FDM}}=1.1_{-0.7}^{+0.83} \times 10^{-19} \mathrm{eV}$, which can be seen in red in Fig. 18. This not only is the strongest constraint to date on $m_{\text {FDM }}$, but it also compatible with most of the other constraints coming from different system. These systems constraint different phenomenology and showed a tendency for a higher FDM mass, confirmed using UFDs in this work.

However, the bounds obtained from dSphs are in tension with all the other bounds on the FDM mass. The bound on the mass obtained for the luminous dSphs, the last row of Fig. 18, falls in the excluded region of almost all of the other bounds, including from UFDs, which are obtained using the same method. This was noted in Safarzadeh and Spergel (2019), where the bounds on the FDM mass using the half-light radius of dSphs and UFDs were compared. They found that the mass obtained using UFDs cannot fit the density profile of Fornax and Sculptor. This was confirmed in Hayashi et al. (2021). This might be an important indication that the FDM model cannot explain the smallscale challenges (this was pointed out in a different context in Burkert (2020)). Or this might indicate several other questions regarding our understanding of how these cores form and their relation to the halo of galaxies. Baryonic effects might change the DM profile in a way that we are not probing the intrinsic DM profile. It could also be that we cannot use an universal density profile for all galaxies, or that we do not understand the relation between the core and halo. Recent simulations (Veltmaat et al. 2018; Schive et al. 2020) have shown that the soliton is not static and oscillates with large amplitudes, which might also impact these numerical fitting functions. This remains an open question and a challenge for the FDM model.

All of these studies show that we need to have better understanding of the modeling of those halos and their formation, and need broader observations and numerical simulations, specially including baryons, to understand and test the FDM class using dwarfs. However, they also show how powerful these small galaxies can be to constrain the FDM. Measuring the density profile of dwarf galaxies is the goal of many future telescopes like PFS and LSST, for example.

\section{Stellar streams}

Stellar streams are a stream of stars orbiting a galaxy which are remnants of a tidally disrupted globular clusters or dwarf galaxies, that was torn apart by a more massive system. These streams are usually thin and very long, extending to dozens of kpc across the 3-dimensions of the halo, and wrap around the disrupting galaxy. Streams are good dynamical probes since they are initially cold and very sensitive to the gravitational potential (Ibata et al. 2002; Johnston et al. 2002; Yoon et al. 2011; Carlberg 2012). This means that the streams when encounter substructures present in the halo of the galaxies can be influenced by it, causing dynamical heating, which are changes in the velocities in the stream, but that are very hard to detect. These encounters also cause disturbances in the morphology of the stream, with the formation of gaps which are underdensities caused by the sub-halos encountered (Carlberg 2012). Only a part of the sub-halos hosts baryonic matter and can be observed directly, so stellar streams offer the opportunity to detect dark halos invisible by the traditional methods 
(see also discussion about lensing below). These gaps contain information about the substructure and can be caused by clumps that are even less massive than a what is expected of a DM sub-halo, showing how sensitive the streams are for detecting substructures. From these gaps, it is possible to infer the properties of the perturber that caused the gap, determining quantities like its mass, scale radius, relative velocity, and impact parameter. It is estimated that we can observe gaps in streams cause by substructures with mass as low as (Erkal and Belokurov 2015; Erkal et al. 2016; Bovy et al. 2017) $M \sim 10^{-5}-10^{-6} M_{\odot}$. This is well below the limit where those halos are expected to host galaxies, and for this reason cannot be probed by usual methods based on detecting the luminous component. On top of that, since the stream extend for large distances in the galaxy and outside the galactic plane, streams might contain detailed information about the gravitational potential and its variations of large part of the halo of a galaxy. With this, stellar streams are an exciting new probe of substructures that can have important consequences in testing different models of DM.

Since different models of DM predict a different amount of sub-halos, it is argued that the stellar streams can be used to test DM models. In the case of the ULDM models, which suppress the formation if substructures, having a much bigger size of minimal subhalo allowed to be created in the galaxy halo. Models like WDM and SIDM also have a modified abundance of sub-halos in comparison with CDM. So there is the hope that these different models would imprint very distinct signals in the streams given their different substructure distributions. Streams can also have gaps coming from baryonic substructures, so one needs to be very careful in the analyses not to overestimate the presence of sub-halos.

Up to now, $22 \mathrm{MW}$ stellar streams are known, being the Sagittarius stream one the most important. ${ }^{46}$ One of the streams that has been used to determine the presence of sub-halos is GD-1. The MW stellar stream GD-1 was discovered using SDSS maps (Grillmair and Dionatos 2006), originated from a globular cluster, and it is seen as a $63^{\circ}$ long structure in the North Galactic region. Gaps of scales of approximately $10^{\circ}$ were found in this stream using SDSS and these were associated with the encounter with sub-halos by Carlberg (2016). This was confirmed by Banik et al. (2021) analyzing the stellar density perturbations from accurate measurements of the morphology (de Boer et al. 2020) of this streams using Gaia data combined with photometry from Pan-STARRS (Chambers et al. 2016). They found that the data indicates that these perturbations should come from sub-halos, and that their abundance and masses are compatible with the expected from CDM from simulations. The error bars of these abundances and expected masses are still large. To detect gaps from subhalos from masses $M \sim 10^{-5}-10^{-7} M_{\odot}$ it is necessary a precision in the determination of the radial velocity of 100-300 m/s. However, these measurements inaugurate and opened the avenue for searches of substructures using stellar streams.

Novel experiments like PFS and LSST promise to measure the streams from the MW and Andromeda in more detail and with higher precision, with the goal of not only detecting the signals of substructures but to understand in more detail the characteristics of the stream, its formation and the properties of its progenitor. Forecasts for LSST (Drlica-Wagner et al. 2019) show that the lowest mass subhalo that the 10-year

\footnotetext{
$\overline{46}$ Around 4 streams are known in Andromeda and 10 streams are known outside the Local group.
} 
LSST data will be able to measure has mass $2 \times 10^{7} M_{\odot}$ for a stellar stream with surface brightness of 33 mag $\operatorname{arcsec}^{-2}$, improving the current bound in three times. LSS can also measure smaller subhalos $\left(10^{-5} M_{\odot}\right)$ since it will allow to access to smaller angular scales, previously dominated by noise, to measure the power spectra of the stream.

However, Ibata et al. (2020) recently revise these conclusions and indicate that the features found in GD-1 can be explained by simple epicyclic motion in a smooth Galactic potential as shown by their N-body simulation, without the need of the presence of a subhalo. Therefore, they conclude that the measurement from GD-1 show no evidence for the influence of DM. This shows that obtaining information about DM substructure in the streams might be more complicated than expected, with some degeneracies with other effects, and that more modelling and understanding of the influences of the MW in the streams are necessary. This is an open topic of research, and all these conclusions need to be studied further and verified by independent groups. But without a doubt, streams are a promising probe for DM, and the next few years are guaranteed to be very exciting in this fast moving field.

\section{Vertical dynamics}

Here we are going to show that the vertical dynamics of stars can be used to test predictions coming from different aspects of the ULDM. We are going to show here that it can be use to probe modifications of the dynamics coming from the MOND behaviour of the theory, in the case of the DM superfluid, or as a way to probe the heating of stars cause by the presence of FDM quasiparticles in the halo. The second effect would also be present in the case of the SIFDM or the DM superfluid since both classes would also present a similar relaxation with macroscopic objects, which can be probed by this effect. But this was only studied in the case of the FDM in the literature, and that is what we present here.

\section{Superfluid DM}

Our galaxy holds more information that can be used to probe DM models. There are several other local MW observables that imprint information from the underlying DM that forms the halo.

One example of one observation that can bring information about the different dynamics imprinted by models like MOND and the DM superfluid is presented in Lisanti et al. (2019a, b). MOND and the DM superfluid model are constructed to give a very good fit to the rotation curves of galaxies and to explain the scaling relations, like the MDAR, by having a different dynamics on small-scales. On top of that, the DM superfluid model reproduces the expected CDM behaviour on large-scales. However, in these papers, the success of those models in explaining the dynamics of galaxies is challenged by observations from the MW dynamics.

The radial dynamics is what it is constrained by those models, since it is the information that enters in the rotation curves of galaxies and the scaling relations. Now, if one consider also the vertical velocities in the vicinity of the Sun, the authors show that then it remains a challenge for those models to explain the rotation curves and these vertical velocity dispersion data. MOND and the DM superfluid, in slightly different ways, modify the dynamics on small scales, where for low-acceleration regions, a 
different acceleration than the Newtonian emerges in the system. In the DM superfluid, this is caused by the presence of the phonons, as we saw above. This modified acceleration changes the dynamics in the radial and in the vertical directions. And in these articles they constraint both the radial and the vertical velocities dispersion for these models in comparison to CDM.

To constraint the radial dynamics, the very precise data from Gaia was used giving data of the circular velocity between $R=[5,18] \mathrm{kpc}$. For the vertical dynamics it was used data of K-dwarfs from the SEGUE sub-survey from SDSS (Sloan Digital Sky Survey) were it was inferred the velocity dispersions for three mono-abundance stellar populations. With this data a Bayesian likelihood analysis was conducted. The authors found a discrepancy between the vertical acceleration predicted by MOND and DM superfluid model in comparison to the one inferred from the data, giving values that are around $15 \%$ larger than the ones inferred from observations and larger than the ones predicted by CDM. And for this reason they claim those models are not preferred as the DM model.

This work establishes an important new observable that should now be taken into account when constructing DM models, their impact not only in the radial dynamics, but also in the vertical dynamics.

However, as some authors have pointed out this result should be taken carefully. McGaugh (2020) pointed out that this is a $2 \sigma$ discrepancy and that maybe the data used to infer the vertical velocities implies a local DM density that is the double of the one inferred from the radial dynamics. This would give a halo that is not spherical, like assumed in the analyses of Lisanti et al. (2019a).

This brings an important point about the data for the vertical dynamics of the MW that we would like to highlight. The dynamics of the MW is very complex with a rich accretion history. Although the MW, with its thin disk, is considered a very stable galaxy with no major recent dynamical interactions, many observations indicate a major accretion event with Sagittarius (Sgr) dwarf spheroidal. This is seen in the streams that wraps around the MW, and it is expected that this event strongly influenced the dynamics of the MW. Laporte et al. (2018) study of the MW's major accretion events with Sgr and the Large Magellanic Clouds (LMC). They show that this encounter with Sagittarius produces oscillations in the MW disk. These vertical perturbations are an influence of many passages of Sgr, as it falls into the MW. The influence of the LMC also changes the dynamics, but much less than Sgr, with Sgr being the main influence in the dynamics of our galaxy in its recent evolution. This shows that the dynamics of the MW is complex and has many unknowns. These vertical oscillations are also found in recent observations both in the Galactic disk and around it, close and far away the solar neighbourhood (see Laporte et al. 2018 for a summary and discussion of those). Therefore, even if the explanation for these oscillations and other features in the Galactic disk is not solely the one above, this rich dynamics of the MW has to be considered when using these observations, specially to constrain models.

In this way, there is the possibility that the vertical dynamics data used in Lisanti et al. $(2019 \mathrm{a}, \mathrm{b})$ has the influence of these oscillations and cannot be used at face value to constraint the dynamics of DM. Or, maybe this complexity of features has to be considered as larger error bars in the inferred vertical dispersion. Therefore, vertical 
velocities are a new and important observable to test DM models, but it necessary to understand if this information can be disentangled from the complex dynamics from the accretion history of the MW. When this degeneracy is resolved this will be an important criteria for DM models to satisfy in the MW.

Hossenfelder and Mistele (2020) also point out that the model of the DM superfluid used is too simplistic and that the DM superfluid interacts with baryons which might also change the vertical accelerations.

\section{FDM}

In the context of the FDM model, the vertical dynamics was also used to constrain the FDM mass in Church et al. (2019), where the change in the vertical dynamics comes from a different effect than the one above. The disk of spiral galaxies is modelled approximately by a decaying exponential for the radial and vertical structure. From observations we see that, in general, we have galaxies that have a thin disk or a thick disk (Binney and Tremaine 2008). What determines the thickness of the disk is the vertical velocity dispersion of the stars in the disk, and its linked to how old the star population is in the disk. As we saw in Sect. 4.1.6, the presence of order one fluctuations in the FDM field, or substructures in the halo, can cause gravitational heating due relaxation between the FDM particles and the stars (Bar-Or et al. 2019). This heating increases the velocity dispersion of the stars, making the size of the system that contains these stars larger: star clusters larger or making the disk in galaxies thicker. In this work they investigate how substructures of the FDM model affect the disk shape. They use simulations that solve the SP system to understand the structure in the FDM model. They find that the subhalos formed in this model, plus the presence of a standing wave in the density profile of FDM, heat the stars in the disk, making the MW disc thicker, with the second effect affecting more the inner disk. They also find the presence of radius dependent flaring of stars caused by these structures. They compare this with measurements of the velocity dispersion of the MW in its thickest part ( $\delta v \sim 32 \mathrm{~km} / \mathrm{s}$ ), and put a bound in the mass of FDM at $2 \sigma$ confidence level of $m>0.6 \times 10^{-22} \mathrm{eV}$.

\section{Dynamical effect: Eridanus II}

In the previous section, we showed how the measurements of the thickness of the disk can probe the velocity dispersion caused by gravitational heating by substructure in the halo in the case DM is the FDM. Here we are going to show that Eridanus II can be used to probe the same effect of the gravitational heating.

The existence of old star cluster like Eridanus II can then be used to constrain these FDM fluctuations, and consequently the mass of the FDM particle. But not only the existence of such sub-halo to host Eridanus II is necessary, but also one needs to check the stability of the star cluster contained in this UFD. It is observed in simulations that the central core formed in the inner part of galaxies has oscillations that changes the density inside the core and might affect star formation. Therefore, if the star cluster region is inside the core, which occurs for $m \lesssim 10^{-20} \mathrm{eV}$, it is going to be affected by the oscillations and might have a different star formation. Otherwise, the star cluster can extend outside the core and can be subject to the interference patterns present in the halo. The effect of those oscillations in the stars is heating of the star cluster, which can disrupt the cluster in a time scale that should have been observed. Taking 
that effect into consideration, if FDM is the total amount of DM, Eridanus II can only form if $m \gtrsim 8 \times 10^{-22} \mathrm{eV}$, which is a value of the mass of the FDM particle that is in slight tension with the one necessary for the FDM model. In Schive et al. (2020) high-resolution simulations, where the random walk behaviour of DM inside the core could be seen, it is shown that Eridanus II is bound to the MW, so its halo might suffer tidal disruption. This reduces the oscillations and counteracts the heating. From that, they claim that the bound obtained in Marsh and Niemeyer (2019), can be evaded and $m \sim 10^{-22} \mathrm{eV}$ is allowed and can explain Eridanus II. These studies show how important is to study the effect of oscillations in the core, and this is the goal of future simulations.

\subsubsection{Substructure-strong lensing}

Different models of DM predict different substructures, from its abundance to the minimum mass for the possible substructures formed. As we saw in this section, generally all the ULDM models suppress the formation of sub-halos of a certain mass and size. By probing the substructures and its properties, then, we can test different models of DM. Therefore, the observables that probe substructures represent one of the most important tests for DM on small-scales.

We already presented above a probe of substructures present in the halo, the stellar streams. This probe seems to be very sensitive to DM substructures and has the potential in the future to help test DM models.

However, there is another way of probing directly the presence of substructures, if they are luminous or not, which is gravitational lensing. Gravitational lensing is distortion of light from objects by the presence of a gravitational potential. One of the main observations used to search for substructures is strong gravitational lensing of quasar. Lensed quasar can present multiple images, arcs or even Einstein rings. The presence of substructures modifies the lensed images of quasar changing its morphology and flux ratios, in a way that the substructure can be mapped, as done in Mao and Schneider (1998), Hsueh et al. (2017), Dalal and Kochanek (2002) and Hezaveh et al. (2016), including distortions from sub-halos in the line of sight (Despali et al. 2018). There are many efforts to probe DM substructures from strong lensing using different frameworks (Vegetti and Koopmans 2009a; Koopmans 2005; Vegetti and Koopmans 2009b; Daylan et al. 2018; Vegetti et al. 2010), and machine learning techniques (Alexander et al. 2020; Brehmer et al. 2019) (for a list of other machine learning approach, see Alexander et al. 2020).

Different models of DM can also predict different types of substructures, like vortices. They can also predict different shapes for these substructures like dark disks. Dark disks are a unique type of substructure in isolated systems, not expected in CDM. Those dark disks can be formed in the models based on the superfluid DM (Alexander et al. 2019) and are very distinct signature of those alternative models. Strong gravitational lensing is an important technique to probe these substructures, and one of the techniques that is going to improve the most with the upcoming experiments.

All the other observations presented before probe substructures or other phenomena from systems that contain stars or galaxies. Gravitational lensing and stellar stream can be used even if the sub-halo or dark structure does not have a luminous component. 
Therefore, these probes offer the possibility of not only probing very light substructures but different dark structures predicted by the ULDM models.

\section{Sub-halo mass function}

As we described in Sect. 4.1.4, the sub-halo mass function for the FDM model presents a redshift dependent cut for smaller sub-halo masses, which leads to a suppression of the small-scale structure. We can then use probes that are sensitive to the gravitational potential to test the SHMF of the FDM model.

One work that attempts to test the suppression of the sub-halos is Schutz (2020). In this work strong lensing, together with stellar streams, is used to constraint the suppressed sub-halos mass function of the FDM model. A bound on the mass of the FDM particle can be obtained, $m \sim 2.1 \times 10^{-21} \mathrm{eV}$, which is again in tension with the value of the mass where the FDM solves the small-scale challenges. A similar analysis was performed in Benito et al. (2020) using a different fitting form for the sub-halo mass function from Schive et al. (2016) and comparable constrain for the FDM mass was obtained $m \sim 5.2 \times 10^{-21} \mathrm{eV}$

\subsection{UV luminosity function}

Here we present constraints on the FDM mass by comparing the predicted cutoff in the luminosity function from the FDM model with observations that probe the UV luminosity function.

The Hubble Ultra Deep Field (HUDF) (Bouwens et al. 2015) is used to search for galaxies at high- $z$. The high- $z$ galaxies measured by the HUDF can be used to match with the expected UV luminosity function predicted by the FDM. This is done in many studies. In Schive et al. (2016), using the HMF fitted from their simulation (161), they used the data from HUDF and found that $m \gtrsim 1.2 \times 10^{-22} \mathrm{eV}(2 \sigma)$, using galaxies from $z=6-8$. A very similar analysis was made in Corasaniti et al. (2017) with similar bounds. In Bozek et al. (2015), this was also analyzed but using the HMF (162) and shows that using the observed UV luminosity from HUDF, a mass of $m=10^{-23} \mathrm{eV}$ is excluded with at $>8 \sigma$, and therefore $m \gtrsim 10^{-22} \mathrm{eV}$ is consistent with HUDF. Obtaining a luminosity function from a full hydrodynamical cosmological simulations of galaxy formation using the initial conditions from the FDM model was done in $\mathrm{Ni}$ et al. (2019), they reach a similar conclusion, ruling out $m<5 \times 10^{-22} \mathrm{eV}(3 \sigma)$. Combining the HUDF data with deep IRAC data from Spitzer Space Telescope over the Great Observatories Origins Deep Survey (GOODS) fields, in Song (2016) they can probe even higher redshifts $z \sim 8$ and show that for $m \sim 10-5 \times 10^{-22} \mathrm{eV}$ the FDM is consistent with their measurements, while $m<2 \times 10^{-22} \mathrm{eV}$ is inconsistent.

One can also use the Hubble Frontier Field (HFF) (Lotz et al. 2017; Koekemoer et al. 2014) that observes the gravitationally lensed ultra-faint galaxies. This observation probes the faint end of the luminosity function. This is exactly where it is expected that the FDM changes the luminosity function, so it is a very good observation to put bounds in the FDM mass. This was done in Menci et al. (2017). Since the measurements that they use are based on gravitational lensing and therefore, it is possible to measure smaller halos, they construct a new luminosity function extending to smaller values of $\mathrm{M}$ in $\Phi\left(<M_{U V}, z\right)=n(>M, z)$. This new luminosity function does not depend on 
baryonic physics that takes place at galaxy formation. With that they are able to place a very strong constraint in the mass of the FDM, which must be $m \gtrsim 8 \times 10^{-22} \mathrm{eV}$ to be compatible with this data. A similar study (Leung et al. 2018) using HFF finds that $m \simeq 10^{-22} \mathrm{eV}$.

\subsection{Black hole superradiance}

Until now we explored ways of probing DM by the gravitational effect they imprint in the structure and substructure of our universe. We explored in detail the astrophysical tests of DM which happen in environments where we are in the weak field regime, but that are dominated by baryonic effects and complex non-linear physics, but that can still give us hints of the nature of DM. We present here a very different way of probing specifically ultra-light fields in strong field environments, far from the linear cosmological scales.

Ultra-light particles can be largely produced around spinning BHs, a process called BH superradiance (Brito et al. 2015b). When a BH rotates faster than the angular phase velocity of an incoming wave, it amplifies the energy and angular momentum of the field in its vicinity. This superradiance effect (Zel'dovich 1971, 1972; Misner 1972; Starobinsky 1973) is a natural mechanism to create clouds of ultralight bosons around Kerr BHs (see Brito et al. 2015b for a review). For ultra-light particles with Compton wavelengths of order or larger than the BHs gravitational radius, they will be efficiently produced by the superradiance, forming a large 'cloud' around the $\mathrm{BH}$. This cloud is a condensate of ultra-light particles created through this instability carrying up to $10 \%$ of the BH's mass and angular momentum, diminishing the initial rotation of the BH (Arvanitaki and Dubovsky 2011; Dolan 2013; Brito et al. 2015a; East and Pretorius 2017).

In the non-relativistic limit, the eigenfunctions of the system are determined by a Schrödinger-like equation and the whole setup is sometimes referred to as a 'gravitational atom'. Superradiance instability depends on the spin of the $\mathrm{BH}$, the mass of the $\mathrm{BH}$ and the mass of the ultra-light particle created, where the modes are corotating with the $\mathrm{BH}$. Depending on the mass of the $\mathrm{BH}$, from stellar mass $\mathrm{BHs}$ to supermassive BHs spanning masses from a few to billions of solar masses, the ultra-light bosons produced through this mechanism can have masses from $10^{-20}$ to $10^{-10} \mathrm{eV}$ (Arvanitaki et al. 2015; Brito et al. 2017; Stott and Marsh 2018). The most stringent constraints to date on the mass of ultra-light bosons using superradiance is presented in Stott and Marsh (2018), where, for the FDM model, masses from $7 \times 10^{-14} \mathrm{eV}<m<2 \times 10^{-11} \mathrm{eV}$ are excluded with $95 \%$ C.L. Using stellar mass BHs, and for SMBHs they are excluded in the range $7 \times 10^{-20} \mathrm{eV}<m<10^{-16} \mathrm{eV}$. These constraints are also valid in the presence of a potential for the axion like mentioned in Sect. 4.1 if $f_{\mathrm{a}} \gtrsim 10^{14} \mathrm{GeV}$. Superradiance can also occur for ultra-light vector fields. In this case, particles of mass $10^{-14} \mathrm{eV}$ to $10^{-11} \mathrm{eV}$ can be created by stellar mass BHs, and $10^{-20} \mathrm{eV}$ to $10^{-17} \mathrm{eV}$ for supermassive BHs (Baryakhtar et al. 2017). The case of spin-2 particles was also studied in Brito et al. (2020).

In Davoudiasl and Denton (2019), the data from the Event Horizon Telescope (EHT) on $\mathrm{M} 87^{*}$ are used to exclude ranges for the mass of scalar ULDM, $2.9 \times 10^{-21} \mathrm{eV}<$ 
$m_{s}<4.6 \times 10^{-21} \mathrm{eV}$, and for vector ULDM, $8.5 \times 10^{-22} \mathrm{eV}<m_{v}<4.6 \times 10^{-21} \mathrm{eV}$. This reference uses the initial EHT data, and it will be interesting to see how this bound will evolve as more data is obtained.

This cloud emits GW, which allows us to probe its presence around BHs. This GW signal could possibly be detected by experiments like LIGO, when coming from stellar BH clouds, and LISA, from supermassive BH clouds (Arvanitaki et al. 2015; Brito et al. 2017; Baryakhtar et al. 2017). The signature of this GW will depend if the cloud is made of real particles, which creates a non-axisymmetric cloud, or complex scalar particles, where the cloud is axisymmetric and the emission of GWs is suppressed. For the cloud made of real bosons given the non-axisymmetric configuration, it can emit GWs when the ultra-light bosons interact with gravitons, or when gravitons change levels, emitting monochromatic GWs (Brito et al. 2017; Bertone et al. 2019). The cloud can also collapse if there is an attractive interaction between those UL particles, emitting GWs in this process.

If the spinning $\mathrm{BH}$ and its respective cloud is in a binary $\mathrm{BH}$ system, the $\mathrm{GW}$ signal is modified due to the presence of the companion presenting a richer $\mathrm{GW}$ phenomenology (Baumann et al. 2019; Hannuksela et al. 2019; Baumann et al. 2020). The evolution and GW signature of the cloud is modified by the presence of a second BH where the waveform and the amplitude of the signal can be modified and even vanish given the resonant transitions between the growing and decaying modes of the cloud. Sharp features in the GW waveform appear, offering a window to probe the signal from these UL particles using GW experiments.

This is an active field of research with the modelling of these effects, the study of back reaction and the observational signatures still ongoing topics of research. Detecting the GWs coming from these clouds would give us the opportunity to probe ultra-light particles in a range that is very interesting for DM. In this way, this observational signature is very relevant to the ULDM models.

As we saw here, those busy and complex environments like galaxies and BHs might still offer, despite their modelling complexity, new ways of probing de fundamental properties of DM. For a review on the same lines for probing fundamental physics in those environments, see Baker et al. (2021).

\subsection{Probing the wave nature of ULDM}

We have shown above the astrophysical and cosmological consequences of DM being described by a BEC or a superfluid inside the halos of galaxies. From changing the behaviour of LSS on small-scales, to changes related to the halos having a minimum mass in those models, and the different profile the wave nature of ULDM leaves in the inner parts of halos, we have been investigating the gravitational consequences of the ULDM models. Some of those predictions might be degenerate with other DM models, like the WDM and SIDM, and even with possible consequences from unaccounted baryonic physics.

However, given the wave nature of the ULDM, these models present some predictions that are a direct consequence of this wave nature and that are completely distinct from any other DM model. We discuss here vortices and interference patterns. These 
are distinct effects that can appear in galactic scales, and can also be connected in the formation of halos.

The detection of any of these effects would be a direct evidence of those models of ULDM and a smoking gun for the wave nature of DM. For this reason, it is interesting to study how these effects arise in each of those classes of ULDM models and understand if they yield observable consequences that allow us to test them.

\subsubsection{Vortices}

Until now, we neglected the fact that the halo might be rotating. As we saw in Sect. 3.5, rotating superfluids have an interesting new phenomenology, the appearance of a lattice of quantized vortices that allow for the rotation of this irrotational fluid. This is a purely quantum mechanical phenomena resulting in quantized vortices being produced. ${ }^{47}$ This new phenomenology arising from rotating BEC halos might lead to observable astrophysical consequences that will represent a direct probe of the wave nature of these DM models. The hope is that measuring this unique signature from these models will make it possible to distinguish this class of models from other alternative DM models which might present some signatures that are degenerate with the ULDM models.

Disk galaxies are one of the most common galaxies in our universe and those are rotationally supported systems. Therefore, the DM halos from those systems are also expected to be rotating. Galaxy halos acquire angular momentum in their formation via tidal torques coming from the neighbouring large-scale structures (Peebles 1969). This angular momentum is conserved after those halos virialize, which leads to the rotating supported disks in galaxies. This process is still being fully understood, with $\mathrm{N}$-body and hydro-simulations showing that the halos of galaxies are expected to have angular momentum. From CDM N-body simulations, the angular momentum obtained, represented by the dimensionless parameter $\lambda=L|E|^{1 / 2} / G M^{5 / 2}$, where $L$ is the angular momentum and $E$ the total energy, are on the range $\lambda \in[0.01,0.1]$ (Barnes and Efstathiou 1987). Therefore, when considering realistic halos of DM, one needs to consider rotation, and if DM is made of ultra-light particles, this can lead to the formation of vortices.

Here we show the effects of the halos being rotating in the ULDM, an effect that is not as explored in the literature as expected, but that might present a decisive observational signature for these ULDM models. We saw in Sect. 3 above, there are conditions for the formation of these vortices, depending on the angular momentum of the rotating halo. The presence of vortices in the DM halo can alter some properties of the halo, like the mass distribution or the presence of those substructures in parts of the halo that might lead to observable signatures. Detectability of these vortices or of the effects caused by their presence, and the observational technique used to probe them, will depend on their abundance and size, which should be studied solving the Schrödinger-Poisson system. Those present some theoretical solutions and estimates of the properties and formation of these vortices, but mainly vortices have to be studied

\footnotetext{
47 It is also worth noticing that a fluid can also form classical vortices, and the difference between those to the quantum vortices is that the quantum vortices are quantized.
} 
numerically, with wave simulations like described above. We present here some of those that investigate this in the SIFDM model, and a rough estimation for the DM superfluid.

During the final preparation of this review a paper studying the possibility of the formation of vortices in gravitationally bounded BECs appeared (Hui et al. 2020). In this paper, they study the possibility of formation of a vortices in the FDM model and of other topological defects with different topologies. They also present results of simulations of this system for rotating halos and the possible observational signatures as consequence of the existence of such vortices.

Other topological defects such as the creation of strings on the condensate in the DM halo in the SIFDM are investigated in Harko and Lake (2015).

\section{Self-interacting $B E C$}

There is a small amount of studies in the literature that investigate the presence of vortices (Rindler-Daller and Shapiro 2012; Zinner 2011; Kain and Ling 2010) (and recently Hui et al. 2020). Given that to investigate vortices one needs to study the Schrödinger-Poisson equations, the main model where those were studied is the simplest superfluid model given by the SIFDM model. But even in these simplified models, it is still hard to find solutions to the GP equation.

Kain and Ling (2010) discuss the possibility of the formation of a lattice of vortices in the halo of a galaxy with the same parameters as Andromeda. This aims to show a order of magnitude estimate for a known galaxy, the amount of vortices formed and put bounds in the mass of the SIFDM particle, and in its interaction. Assuming that a galaxy like M31 has $M \sim 10^{12} M_{\odot}$, average radius $R \sim 100 \mathrm{kpc}$, average density $\rho_{\text {avg }} \sim 10^{-23} \mathrm{~kg} / \mathrm{m}^{3}$ and angular velocity $\Omega \sim 10^{-16} \mathrm{rad} / \mathrm{s}$. If vortices are created they cannot be larger than the size of the condensate core, $R_{\mathrm{v}} \leq R_{\mathrm{bec}}$, the SIFDM must have a mass $m \gtrsim 10^{-24} \mathrm{eV}$, with radius $R_{\mathrm{V}} \sim 10^{21} \mathrm{~m} \approx 30 \mathrm{kpc}$. Therefore, in a halo like the halo of M31, there might be from 1 to 100 vortices. For the simplified case of having only one vortex in the halo, the GP equation can be solved in the ThomasFermi limit, ignoring the QP term, determining the structure of this cylindrical vortex core, and the critical velocity, showing that Andromeda galaxy could have formed a vortex given its angular velocity.

Zinner (2011) focuses in a simplified study of the consequences for virialization and on the rotation curves if a lattice of vortices is present in the FDM model. Depending on the choice of mass and interaction, this vortices can lead to oscillations in the rotation curve of galaxies, which the authors claim resemble what is observed in rotation curve of spiral galaxies.

Now, Rindler-Daller and Shapiro (2012) try to study the vortex solutions in more detail, solving the GP for some assumed halo profiles that are more realistic than in the previous work (see also Suárez et al. 2014 for a review of vortices in SIFDM).

First, it is interesting to repeat something we presented in Sect. 3.5. The presence of a rotation in a condensate in a spherical halo is that without rotation, the BEC wavefunction is real and positive; however, the angular velocity induces a superfluid current, making the wavefunction to be complex, $\psi=|\phi| e^{i S(t, \mathbf{r})}$, with a phase that gives a velocity flow, $v=\hbar \nabla S / m$, and as we saw in Sect. 3.5, the fluid velocity is $\mathbf{v}^{\prime}=\mathbf{v}-\times \mathbf{v}$. Therefore, a rotating BEC is a superfluid. As shown in Table 1 from 
Rindler-Daller and Shapiro (2012), FDM models, where there is no interactions, do not form vortices. If the interaction is attractive, vortices are also not formed. For this reason, we work in the case of SIFDM model with repulsive interaction.

The condition for the formation of vortices is $\Omega>\Omega_{\mathrm{c}}$ or equivalently $L>L_{\mathrm{qm}}$. This imposes a bound in the mass of the SIFDM particles. They work this out and the solutions for the GP equations for two halo models. First, in halo model A, they assume a simplified halo model where the density and potential is given by a homogeneous Maclaurin spheroid, which gives a known form for the gravitational potential inside halos. This halo is not irrotational, having $L \gg L_{\mathrm{qm}}$. With that simplified gravitational potential one can calculate the characteristics of a virialized rotating halo. This allows to put bounds in the mass and the interaction of the SIFDM particle, assuming the critical case where $\left(L / L_{\mathrm{qm}}\right)_{\mathrm{c}}$ and $\lambda=0.05$ is the average of the dimensionless rotation parameter: $m / m_{\mathrm{H}} \geq\left(\mathrm{m} / m_{\mathrm{H}}\right)_{\mathrm{c}} \approx 50$ and $\mathrm{g} / \mathrm{g}_{\mathrm{H}} \geq\left(\mathrm{g} / \mathrm{g}_{\mathrm{H}}\right)_{\mathrm{c}} \approx 2550$.

For a more realistic halo which allows to take into account the compressibility of the fluid, the halo is considered as $(n=1)$-polytropic Riemann-S ellipsoids, halo model $B$, which is irrotational before forming halos $L=L_{\mathrm{qm}}$. This shows that if $L<L_{\mathrm{qm}}$, there is no formation of vortices and the rotating BEC can be described by halo model $B$. And for $L \gg L_{\mathrm{qm}}$, there is the formation of, at least one vortex if the quantities are equal to the critical quantities or more in case they exceed these values, with the halo being described by halo model $A$. The case where $L \gtrsim L_{\mathrm{qm}}$, the halo is described by the halo model $B$ again, with a single vortex in the center of the halo.

This study shows the conditions for the formation of the vortices and shows the complexity that can arise in the presence of rotations and of a vortex lattice.

These studies show important characteristics for the formation of vortices in the halo of galaxies in the SIFDM model. However, to fully study the presence and consequences of a rotating halo in those models one needs to perform wave simulations. One study that takes that in consideration is Hui et al. (2020).

\section{DM superfluid}

As we saw above, to calculate the abundance and properties of the vortices, it is necessary to solve the equations of motion of the superfluid coupled to the Poisson equation. For the DM superfluid model, there is still no numerical study of the solution of the equations equivalent to the GP in the presence of gravity to understand the formation of vortices in this model. For this reason, here we present a dimensional analysis and order of magnitude estimation of the presence of vortices in the DM superfuid context.

Vortices are formed when the angular velocity of the superfluid is larger than the critical velocity: $\Omega_{\mathrm{Sf}} \gg \Omega_{\mathrm{c}}$, with the critical angular velocity given by (80). For $R \sim 100 \mathrm{kpc}$ and $m \sim \mathrm{eV}$, we can see that $\Omega_{\mathrm{c}} \sim 10^{-41} \mathrm{~s}^{-1}$ (neglecting the logarithmic factor). This is much smaller than the rotation velocity of the halo: $\Omega \sim \lambda \sqrt{G \rho} \sim$ $10^{-18} \mathrm{~s}^{-1}$, using a halo mass density $\rho \sim 10^{-25} \mathrm{~g} / \mathrm{cm}^{3}$. So, there will be the production of vortices in the halos of the galaxies in the DM superfluid model And this production seem to be very numerous.

We can estimate the number of vortices in the halo $N_{v}=\Omega / \Omega_{\mathrm{c}} \sim 10^{23}$, with a core radius, given by the healing length, $\xi=1 /\left(m c_{\mathrm{S}}\right) \sim \mathrm{mm}$ (assumed a MW type galaxy and fiducial values). Although highly numbered, these vortices are small. It 

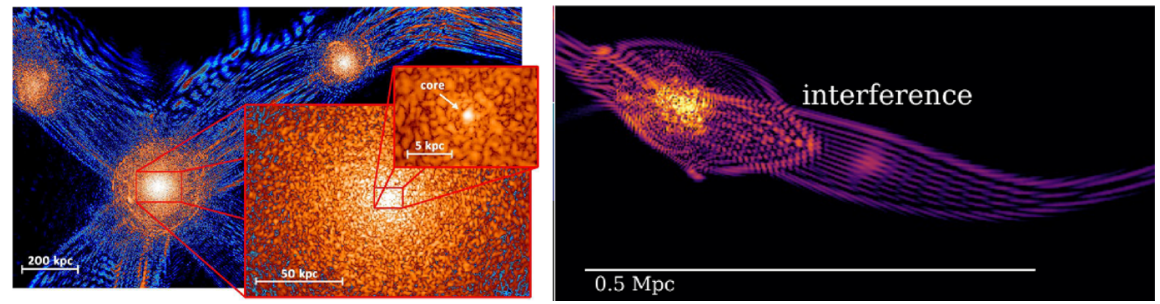

Fig. 21 In this figure, we show the interference pattern in the DM halos from hydrodynamical simulations of the fuzzy DM model. Left panel: the density distribution of FDM at redshift $z=0.1$ at different scales. This simulation uses the GAMER code to describe the wave-like FDM using an adaptive-mesh-refinement (AMR) scheme. Image reproduced by permission from Schive et al. (2014a), copyright by Macmillan. Right panel: simulations of the slices of DM through a filament in the DM distribution at redshift $z=5.5$. This simulation was made by modifying the magneto-hydrodynamics code AREPO for FDM model. These figures clearly show the interference pattern that the FDM model imprints in the halos which is very distinct from CDM or even other DM models like WDM. These interference fringes that are present at the scales of the de Broglie wavelength. On top of that, in both stimulations we can see the soliton cores formed in the halos, also characteristic of the FDM model. Image reproduced with permission from Mocz et al. (2019), copyright by APS

is still unclear if it is possible to detect those vortices via, for example, gravitational lensing or any other effect they might have in the galaxy.

\subsubsection{Interference fringes}

Another interesting effect that comes from the wave nature of DM superfluid model is interference. Since the condensate is described by a coherent wave function with the density if give by $|\psi|^{2}$, in the formation of galaxies, interference patterns are expected to form.

As we discussed in the numerical simulations section, the interference patterns can only be seen in wave simulations since when there is destructive interference, the quantum pressure term is not well defined and this is not present in the fluid simulations. We show in Fig. 21, the result of two wave simulations from different groups that show the interference fringes that appear in the filaments of FDM structure. These patterns appear on scales of order of the de Broglie wavelength. General features like caustics, which are density singularities that usually appear in CDM and even WDM simulations, are regularized in FDM models due to the uncertainty principle, and do not appear in FDM simulations.

Another effect that could generate interference patterns is the collision of subsonic galaxies. Since those galaxies maintain the coherence of their condensate core described by the coherent wave function, the collision between these cores leaves an interference pattern (Maleki et al. 2020). If this interference fringes could be observed, this would be still another form of probing these models.

Interference patterns are going to be created also in the merging processes of dark/bright solitons (Hamner et al. 2011; González and Guzmán 2011). Some authors suggest that this effect could be linked to the shells seen around elliptical galaxies (Cooper et al. 2011). 


\section{Summary}

In this review, we studied an alternative class of DM model, the ultra-light DM. These models have been receiving a lot of attention in the literature nowadays given their interesting property of forming a BEC or superfluid on galactic scales. In this review, we aimed to give a summary of the models of ULDM, suggesting for the first time a classification into three categories according to the their non-relativistic behaviour and the structures they form in galaxies: the fuzzy DM, self-interacting BEC and the DM superfluid. Their different descriptions lead to different phenomenologies and observational effects that can be used to test these DM paradigms. We had also the goal to give a snapshot of the field as it stands at present. We saw that current observations highly constrain the mass of the FDM and, if these constrains hold, the mass range which shows an interesting phenomenology on small-scales and reconciles these scales with the CDM successes on large-scales is challenged for this model.

We highlight how important the observations on small-scales are for helping to determine the nature of DM, and how the observations of the small-scales, galaxies and our MW have been advancing very fast in the last few years. With current and new experiments like Gaia, LSST, PFS, HERA, SKA, just to cite a few, and the new and exciting probes like stellar streams, $21 \mathrm{~cm}$ cosmology, MW observables, $\mathrm{BH}$ superradiance, the next few years promise to revolutionize the tests of DM on small-scales.

In this review we tried also to give a complete description of the theory behind these models, describing the striking phenomena of BEC and superfluidity. We stressed the difference between models, showing their different descriptions and phenomenological consequences. Since the understanding of this DM class of models requires knowledge from several fields, including condensed matter physics, we believe that this theoretical summary of these constructions, is very important to better understand all the features that these models of DM can present and even help in finding new observables for these models. It is also important for future progress since there is still a lot of room for theoretical development of these DM models.

Maybe one of the biggest challenge for these models and testing them against observations is the need for numerical simulations. Those are necessary so we can understand how the formation of structures proceeds in these models. However, performing those simulations in a way that they resolve the small-scales in order for us to see the interesting effects coming from the wave nature of these models, and also that they simulate the structure on cosmological scales is a challenge. Incredible advances in this field have been made in the past few years and there are many groups currently working to improve those simulations. Most of those advance, though, are only for the FDM model. Simulations for the SIBEC are only a few and there is still no numerical framework to study the DM superfluid. Thus, we should expect continue progress in this field in the future, which will lead to also a better understanding on how to probe those models with observations.

In summary, the study of the ultra-light DM is an active area of research and many challenges are still opened to be addressed theoretically, numerically and observationally. As this field becomes more and more popular, we believe this progress will go 
even faster and we hope this review can help those entering or already in this field supporting their understanding of this fascinating new DM model.

Acknowledgements First, I would like to thank The Astronomy and Astrophysics Review for the invitation to write this review. I am very grateful to Eiichiro Komatsu for the help, guidance and encouragement for writing this review. I thank Robert Brandenberger for all the advice and comments that were essential to completion of this work. I am also very grateful to Evan McDonough, Jerome Quintin and Guilherme Franzmann for carefully reading and useful comments to the content of the review, and Jerome for the thorough proofreading. Thanks to Justin Khoury for supporting this project, and to both Lasha Berezhiani and Justin for teaching me about this topic so patiently. I would like to thank Eduardo Toshio Matsushita for teaching so much about BECs and quantum mechanics. I also thank the referee for the useful comments and suggestions that helped improve the review.

Funding Open Access funding enabled and organized by Projekt DEAL.

Open Access This article is licensed under a Creative Commons Attribution 4.0 International License, which permits use, sharing, adaptation, distribution and reproduction in any medium or format, as long as you give appropriate credit to the original author(s) and the source, provide a link to the Creative Commons licence, and indicate if changes were made. The images or other third party material in this article are included in the article's Creative Commons licence, unless indicated otherwise in a credit line to the material. If material is not included in the article's Creative Commons licence and your intended use is not permitted by statutory regulation or exceeds the permitted use, you will need to obtain permission directly from the copyright holder. To view a copy of this licence, visit http://creativecommons.org/licenses/by/4.0/.

\section{References}

Abazajian K, et al. (2019) CMB-S4 science case, reference design, and project plan. arXiv:1907.04473 [astro-ph.IM] (arXiv e-prints)

Abbott L, Sikivie P (1983) A cosmological bound on the invisible axion. Phys Lett B 120:133-136. https:// doi.org/10.1016/0370-2693(83)90638-X

Abbott BP et al (2017) GW170817: observation of gravitational waves from a binary neutron star inspiral. Phys Rev Lett 119(16):161101. https://doi.org/10.1103/PhysRevLett.119.161101. arXiv:1710.05832 [gr-qc]

Abo-Shaeer JR, Raman C, Vogels JM, Ketterle W (2001) Observation of vortex lattices in Bose-Einstein condensates. Science 292(5516):476-479. https://doi.org/10.1126/science.1060182

Ade PAR et al (2016) Planck 2015 results XIII. Cosmological parameters. Astron Astrophys 594:A13. https://doi.org/10.1051/0004-6361/201525830. arXiv:1502.01589 [astro-ph.CO]

Akeson R (2019) The wide field infrared survey telescope: 100 Hubbles for the 2020s. arXiv:1902.05569 [astro-ph.IM] (arXiv e-prints)

Alexander S, McDonough E, Spergel DN (2018) Chiral gravitational waves and baryon superfluid dark matter. JCAP 05:003. https://doi.org/10.1088/1475-7516/2018/05/003. arXiv:1801.07255 [hep-th]

Alexander S, Bramburger JJ, McDonough E (2019) Dark disk substructure and superfluid dark matter. Phys Lett B 797:134871. https://doi.org/10.1016/j.physletb.2019.134871. arXiv:1901.03694 [astro-ph.CO]

Alexander S, Gleyzer S, McDonough E, Toomey MW, Usai E (2020) Deep learning the morphology of dark matter substructure. Astrophys J 893:15. https://doi.org/10.3847/1538-4357/ab7925. arXiv:1909.07346 [astro-ph.CO]

Allali I, Hertzberg MP (2020) Gravitational decoherence of dark matter. JCAP 07:056. https://doi.org/10. 1088/1475-7516/2020/07/056. arXiv:2005.12287 [gr-qc]

Allen JF, Misener AD (1938) Flow of liquid helium II. Nature 141(3558):75. https://doi.org/10.1038/ $141075 \mathrm{a} 0$

Allgood B, Flores RA, Primack JR, Kravtsov AV, Wechsler RH, Faltenbacher A, Bullock JS (2006) The shape of dark matter haloes: dependence on mass, redshift, radius and formation. MNRAS 367(4):17811796. https://doi.org/10.1111/j.1365-2966.2006.10094.x. arXiv:astro-ph/0508497 [astro-ph] 
Amin MA, Mocz P (2019) Formation, gravitational clustering, and interactions of nonrelativistic solitons in an expanding universe. Phys Rev D 100(6):063507. https://doi.org/10.1103/PhysRevD.100.063507. arXiv:1902.07261 [astro-ph.CO]

Amin MA, Easther R, Finkel H, Flauger R, Hertzberg MP (2012) Oscillons after inflation. Phys Rev Lett 108:241302. https://doi.org/10.1103/PhysRevLett.108.241302. arXiv:1106.3335 [astro-ph.CO]

Anderson MH, Ensher JR, Matthews MR, Wieman CE, Cornell EA (1995) Observation of Bose-Einstein condensation in a dilute atomic vapor. Science 269:198-201. https://doi.org/10.1126/science.269. 5221.198

Anderson Let al (2014) The clustering of galaxies in the SDSS-III Baryon Oscillation Spectroscopic Survey: baryon acoustic oscillations in the Data Releases 10 and 11 Galaxy samples. MNRAS 441(1):24-62. https://doi.org/10.1093/mnras/stu523. arXiv:1312.4877 [astro-ph.CO]

Arbey A, Lesgourgues J, Salati P (2003) Galactic halos of fluid dark matter. Phys Rev D 68:68-023511. https://doi.org/10.1103/PhysRevD.68.023511. arXiv:astro-ph/0301533

Arcadi G, Dutra M, Ghosh P, Lindner M, Mambrini Y, Pierre M, Profumo S, Queiroz FS (2018) The waning of the WIMP? A review of models, searches, and constraints. Eur Phys J C 78(3):203. https://doi.org/ 10.1140/epjc/s10052-018-5662-y. arXiv:1703.07364 [hep-ph]

Arias P, Cadamuro D, Goodsell M, Jaeckel J, Redondo J, Ringwald A (2012) WISPy cold dark matter. JCAP 06:013. https://doi.org/10.1088/1475-7516/2012/06/013. arXiv:1201.5902 [hep-ph]

Armengaud E, Palanque-Delabrouille N, Yèche C, Marsh DJE, Baur J (2017) Constraining the mass of light bosonic dark matter using SDSS Lyman- $\alpha$ forest. MNRAS 471(4):4606-4614. https://doi.org/ 10.1093/mnras/stx1870. arXiv:1703.09126 [astro-ph.CO]

Arvanitaki A, Dubovsky S (2011) Exploring the string axiverse with precision black hole physics. Phys Rev D 83:044026. https://doi.org/10.1103/PhysRevD.83.044026. arXiv:1004.3558 [hep-th]

Arvanitaki A, Dimopoulos S, Dubovsky S, Kaloper N, March-Russell J (2010) String axiverse. Phys Rev D 81:123530. https://doi.org/10.1103/PhysRevD.81.123530. arXiv:0905.4720 [hep-th]

Arvanitaki A, Baryakhtar M, Huang X (2015) Discovering the QCD axion with black holes and gravitational waves. Phys Rev D 91(8):084011. https://doi.org/10.1103/PhysRevD.91.084011. arXiv:1411.2263 [hep-ph]

Arvanitaki A, Dimopoulos S, Galanis M, Lehner L, Thompson JO, Van Tilburg K (2020) Largemisalignment mechanism for the formation of compact axion structures: signatures from the QCD axion to fuzzy dark matter. Phys Rev D 101(8):083014. https://doi.org/10.1103/PhysRevD.101. 083014. arXiv:1909.11665 [astro-ph.CO]

Bailin J, Steinmetz M (2005) Internal and external alignment of the shapes and angular momenta of $\Lambda$ CDM halos. Astrophys J 627(2):647-665. https://doi.org/10.1086/430397. arXiv:astro-ph/0408163 [astroph]

Baker J, George A (1999) Neutron matter model. Phys Rev C 60(5). https://doi.org/10.1103/PhysRevC.60. 054311

Baker T, et al. (2021) Novel probes project: tests of gravity on astrophysical scales. Rev Mod Phys arXiv:1908.03430 [astro-ph.CO]

Ballagh RJ, Burnett K, Scott TF (1997) Theory of an output coupler for Bose-Einstein condensed atoms. Phys Rev Lett 78(9):1607-1611. https://doi.org/10.1103/PhysRevLett.78.1607

Banik N, Bovy J, Bertone G, Erkal D, de Boer TJL (2021) Evidence of a population of dark subhalos from Gaia and Pan-STARRS observations of the GD-1 stream. MNRAS 502(2):2364-2380. https://doi.org/ 10.1093/mnras/stab210 arXiv:1911.02662 [astro-ph.GA]

Bar-Or B, Fouvry JB, Tremaine S (2019) Relaxation in a fuzzy dark matter halo. Astrophys J 871(1):28. https://doi.org/10.3847/1538-4357/aaf28c. arXiv:1809.07673 [astro-ph.GA]

Bardeen JM, Bond JR, Kaiser N, Szalay AS (1986) The statistics of peaks of Gaussian random fields. Astrophys J 304:15. https://doi.org/10.1086/164143

Barenghi CF, Parker NG (2016) A Primer on Quantum Fluids. SpringerBriefs in Physics. Springer, Cham. https://doi.org/10.1007/978-3-319-42476-7. arXiv:1605.09580 [cond-mat.quant-gas]

Barenghi CF, Donnelly RJ, Vinen WF (2001) Quantized vortex dynamics and superfluid turbulence. Lecture notes in physics, vol 571. Springer, Berlin. https://doi.org/10.1007/3-540-45542-6

Barkana R, Haiman Z, Ostriker JP (2001) Constraints on warm dark matter from cosmological reionization. Astrophys J 558:482. https://doi.org/10.1086/322393. arXiv:astro-ph/0102304

Barnes J, Efstathiou G (1987) Angular momentum from tidal torques. Astrophys J 319:575. https://doi.org/ $10.1086 / 165480$ 
Barranco J, Bernal A (2011) Self-gravitating system made of axions. Phys Rev D 83:043525. https://doi. org/10.1103/PhysRevD.83.043525. arXiv:1001.1769 [astro-ph.CO]

Baryakhtar M, Lasenby R, Teo M (2017) Black hole superradiance signatures of ultralight vectors. Phys Rev D 96(3):035019. https://doi.org/10.1103/PhysRevD.96.035019. arXiv:1704.05081 [hep-ph]

Battesti R, Beltran B, Davoudiasl H, Kuster M, Pugnat P, Rabadán R, Ringwald A, Spooner N, Zioutas $\mathrm{K}$ (2008) Axion searches in the past, at present, and in the near future. In: Kuster M, Raffelt G, Beltran B (eds) Axions: theory, cosmology, and experimental searches. Lecture notes in physics, vol 741. Springer, Berlin, pp 199-237. https://doi.org/10.1007/978-3-540-73518-2_10. arXiv:0705.0615 [hep-ex]

Bauer JB, Marsh DJ, Hložek R, Padmanabhan H, Laguë A (2020) Intensity mapping as a probe of axion dark matter. arXiv:2003.09655 [astro-ph.CO] (arXiv e-prints)

Baumann D, Chia HS, Porto RA (2019) Probing ultralight bosons with binary black holes. Phys Rev D 99(4):044001. https://doi.org/10.1103/PhysRevD.99.044001. arXiv:1804.03208 [gr-qc]

Baumann D, Chia HS, Porto RA, Stout J (2020) Gravitational collider physics. Phys Rev D 101(8). https:// doi.org/10.1103/PhysRevD.101.083019. arXiv:1912.04932 [gr-qc]

Beekman AJ, Rademaker L, van Wezel J (2019) An introduction to spontaneous symmetry breaking. SciPost Phys Lect Notes. https://doi.org/10.21468/SciPostPhysLectNotes.11. arXiv:1909.01820 [hep-th]

Begeman KG, Broeils AH, Sanders RH (1991) Extended rotation curves of spiral galaxies: dark haloes and modified dynamics. MNRAS 249:523

Bekenstein J, Milgrom M (1984) Does the missing mass problem signal the breakdown of Newtonian gravity? Astrophys J 286:7-14. https://doi.org/10.1086/162570

Beliaev ST (1958) Energy spectrum of a non-ideal Bose gas. Sov Phys JETP 34(2):299

Benito M, Criado JC, Hütsi G, Raidal M, Veermäe H (2020) Implications of Milky Way substructures for the nature of dark matter. Phys Rev D 101(10):103023. https://doi.org/10.1103/PhysRevD.101.103023. arXiv:2001.11013 [astro-ph.CO]

Benson AJ, Lacey CG, Baugh CM, Cole S, Frenk CS (2002) The effects of photoionization on galaxy formation-I. Model and results at $z=0$. MNRAS 333:156. https://doi.org/10.1046/j.1365-8711. 2002.05387.x. arXiv:astro-ph/0108217

Berezhiani L (2020) On effective theory of superfluid phonons. Phys Lett B 805:135451. https://doi.org/ 10.1016/j.physletb.2020.135451. arXiv:2001.08696 [hep-th]

Berezhiani L, Khoury J (2015) Theory of dark matter superfluidity. Phys Rev D 92:103510. https://doi.org/ 10.1103/PhysRevD.92.103510. arXiv:1507.01019 [astro-ph.CO]

Berezhiani L, Khoury J (2016) Dark matter superfluidity and galactic dynamics. Phys Lett B 753:639-643. https://doi.org/10.1016/j.physletb.2015.12.054. arXiv:1506.07877 [astro-ph.CO]

Berezhiani L, Famaey B, Khoury J (2018) Phenomenological consequences of superfluid dark matter with baryon-phonon coupling. JCAP 09:021. https://doi.org/10.1088/1475-7516/2018/09/021. arXiv:1711.05748 [astro-ph.CO]

Berezhiani L, Elder B, Khoury J (2019) Dynamical friction in superfluids. JCAP 10:074. https://doi.org/ 10.1088/1475-7516/2019/10/074. arXiv:1905.09297 [hep-ph]

Bertone G, Hooper D (2018) History of dark matter. Rev Mod Phys 90(4):045002. https://doi.org/10.1103/ RevModPhys.90.045002. arXiv:1605.04909 [astro-ph.CO]

Bertone G, et al. (2019) Gravitational wave probes of dark matter: challenges and opportunities. SciPost Phys Core 3, 007 (2020). https://doi.org/10.21468/SciPostPhysCore.3.2.007. arXiv:1907.10610 [astro-ph.CO]

Bhupal Dev PS, Mazumdar A, Qutub S (2014) Constraining non-thermal and thermal properties of dark matter. Front Phys 2:26. https://doi.org/10.3389/fphy.2014.00026. arXiv:1311.5297 [hep-ph]

Binney J, Tremaine S (2008) Galactic dynamics, 2nd edn. Princeton University Press, Princeton

Boehmer CG, Harko T (2007) Can dark matter be a Bose-Einstein condensate? JCAP 06:025. https://doi. org/10.1088/1475-7516/2007/06/025. arXiv:0705.4158 [astro-ph]

Bohm D (1952a) A suggested interpretation of the quantum theory in terms of hidden variables. 1. Phys Rev 85:166-179. https://doi.org/10.1103/PhysRev.85.166

Bohm D (1952b) A suggested interpretation of the quantum theory in terms of hidden variables. 2. Phys Rev 85:180-193. https://doi.org/10.1103/PhysRev.85.180

Boran S, Desai S, Kahya EO, Woodard RP (2018) GW170817 falsifies dark matter emulators. Phys Rev D 97(4):041501. https://doi.org/10.1103/PhysRevD.97.041501. arXiv:1710.06168 [astro-ph.HE] 
Bornheimer U, Vasić I, Hofstetter W (2017) Phase transitions of the coherently coupled two-component Bose gas in a square optical lattice. Phys Rev A 96(6):063623. https://doi.org/10.1103/PhysRevA.96. 063623. arXiv:1705.02833 [cond-mat.quant-gas]

Borriello A, Salucci P (2001) The dark matter distribution in disk galaxies. MNRAS 323:285. https://doi. org/10.1046/j.1365-8711.2001.04077.x. arXiv:astro-ph/0001082

Borzyszkowski M, Porciani C, Romano-Diaz E, Garaldi E (2017) ZOMG-I. How the cosmic web inhibits halo growth and generates assembly bias. MNRAS 469(1):594-611. https://doi.org/10.1093/mnras/ stx873. arXiv:1610.04231 [astro-ph.CO]

Bose SN (1924) Planck's law and light quantum hypothesis. Z Phys 26:178-181. https://doi.org/10.1007/ BF01327326

Bouwens R et al (2015) UV luminosity functions at redshifts $z \sim 4$ to $z \sim 10$ : 10000 galaxies from HST legacy fields. Astrophys J 803(1):34. https://doi.org/10.1088/0004-637X/803/1/34. arXiv:1403.4295 [astro-ph.CO]

Bovill MS, Ricotti M (2009) Pre-reionization fossils, ultra-faint dwarfs and the missing galactic satellite problem. Astrophys J 693:1859-1870. https://doi.org/10.1088/0004-637X/693/2/1859. arXiv:0806.2340 [astro-ph]

Bovy J, Erkal D, Sanders JL (2017) Linear perturbation theory for tidal streams and the smallscale CDM power spectrum. MNRAS 466(1):628-668. https://doi.org/10.1093/mnras/stw3067. arXiv: 1606.03470 [astro-ph.GA]

Bowman JD, Rogers AEE, Monsalve RA, Mozdzen TJ, Mahesh N (2018) An absorption profile centred at 78 megahertz in the sky-averaged spectrum. Nature 555(7694):67-70. https://doi.org/10.1038/ nature25792. arXiv:1810.05912 [astro-ph.CO]

Boylan-Kolchin M, Bullock JS, Kaplinghat M (2011) Too big to fail? The puzzling darkness of massive Milky Way subhaloes. MNRAS 415:L40. https://doi.org/10.1111/j.1745-3933.2011.01074.x. arXiv: 1103.0007 [astro-ph.CO]

Boylan-Kolchin M, Bullock JS, Kaplinghat M (2012) The Milky Way's bright satellites as an apparent failure of LCDM. MNRAS 422:1203-1218. https://doi.org/10.1111/j.1365-2966.2012.20695.x. arXiv:1111.2048 [astro-ph.CO]

Bozek B, Marsh DJE, Silk J, Wyse RFG (2015) Galaxy UV-luminosity function and reionization constraints on axion dark matter. MNRAS 450(1):209-222. https://doi.org/10.1093/mnras/stv624. arXiv:1409.3544 [astro-ph.CO]

Braaten E, Mohapatra A, Zhang H (2016) Dense axion stars. Phys Rev Lett 117:12. https://doi.org/10.1103/ PhysRevLett.117.121801. arXiv:1512.00108 [hep-ph]

Brehmer J, Mishra-Sharma S, Hermans J, Louppe G, Cranmer K (2019) Mining for dark matter substructure: inferring Subhalo population properties from strong lenses with machine learning. Astrophys J. https:// doi.org/10.3847/1538-4357/ab4c41. arXiv:1909.02005 [astro-ph.CO]

Brito R, Cardoso V, Pani P (2015a) Black holes as particle detectors: evolution of superradiant instabilities. Class Quantum Grav 32(13):134001. https://doi.org/10.1088/0264-9381/32/13/134001. arXiv: 1411.0686 [gr-qc]

Brito R, Cardoso V, Pani P (2015b) Superradiance: energy extraction, black-hole bombs and implications for astrophysics and particle physics, Lecture Notes in Physics, vol 906. Springer, Cham. https://doi. org/10.1007/978-3-319-19000-6. arXiv:1501.06570 [gr-qc]

Brito R, Ghosh S, Barausse E, Berti E, Cardoso V, Dvorkin I, Klein A, Pani P (2017) Gravitational wave searches for ultralight bosons with LIGO and LISA. Phys Rev D 96(6):064050. https://doi.org/10. 1103/PhysRevD.96.064050. arXiv:1706.06311 [gr-qc]

Brito R, Grillo S, Pani P (2020) Black hole superradiant instability from ultralight spin-2 fields. Phys Rev Lett 124(21):211101. https://doi.org/10.1103/PhysRevLett.124.211101. arXiv:2002.04055 [gr-qc]

Broeils A (1992) Dark and visible matter in spiral galaxies. PhD thesis, University of Groningen

Bryan GL, Norman ML (1998) Statistical properties of X-ray clusters: analytic and numerical comparisons. Astrophys J 495:80. https://doi.org/10.1086/305262. arXiv:astro-ph/9710107

Bullock JS, Boylan-Kolchin M (2017) Small-scale challenges to the $\Lambda$ CDM paradigm. Annu Rev Astron Astrophys 55:343-387. https://doi.org/10.1146/annurev-astro-091916-055313. arXiv:1707.04256 [astro-ph.CO]

Bullock JS, Kravtsov AV, Weinberg DH (2000) Reionization and the abundance of galactic satellites. Astrophys J 539:517. https://doi.org/10.1086/309279. arXiv:astro-ph/0002214 
Burgess CP (2000) Goldstone and pseudo-Goldstone bosons in nuclear, particle and condensed matter physics. Phys Rep 330:193-261. https://doi.org/10.1016/S0370-1573(99)00111-8. arXiv:hep-th/9808176

Burkert A (2020) Fuzzy dark matter and dark matter halo cores. Astrophys J 904(2):161. https://doi.org/ 10.3847/1538-4357/abb242. arXiv:2006.11111 [astro-ph.GA]

Cai RG, Liu TB, Wang SJ (2018) Gravitational wave as probe of superfluid dark matter. Phys Rev D 97:2. https://doi.org/10.1103/PhysRevD.97.023027. arXiv:1710.02425 [hep-ph]

Calabrese E, Spergel DN (2016) Ultra-light dark matter in ultra-faint dwarf galaxies. MNRAS 460(4):43974402. https://doi.org/10.1093/mnras/stw1256. arXiv:1603.07321 [astro-ph.CO]

Cappellaro A, Macrı T, Bertacco GF, Salasnich L (2017) Equation of state and self-bound droplet in Rabi-coupled Bose mixtures. Sci Rep 7:13358. https://doi.org/10.1038/s41598-017-13647-y. arXiv:1705.01814 [cond-mat.quant-gas]

Carlberg RG (2012) Dark matter sub-halo counts via star stream crossings. Astrophys J 748:20. https://doi. org/10.1088/0004-637X/748/1/20. arXiv:1109.6022 [astro-ph.CO]

Carlberg RG (2016) Modeling GD-1 gaps in a milky way potential. Astrophys J 820(1):45. https://doi.org/ 10.3847/0004-637X/820/1/45. arXiv:1512.01620 [astro-ph.GA]

Carr B, Kuhnel F (2020) Primordial black holes as dark matter: recent developments. Annu Rev Nucl Part Sci. https://doi.org/10.1146/annurev-nucl-050520-125911. arXiv:2006.02838 [astro-ph.CO]

Carr B, Kohri K, Sendouda Y, Yokoyama J (2020) Constraints on primordial black holes. arXiv:2002.12778 [astro-ph.CO] (arXiv e-prints)

Carroll SM (1999) TASI lectures: cosmology for string theorists. In: Theoretical advanced study institute in elementary particle physics (TASI 99): strings, branes, and gravity, pp 437-492. https://doi.org/10. 1142/9789812799630_0004. arXiv:hep-th/0011110

Castellanos E, Escamilla-Rivera C, Macías A, Núñez D (2014) Scalar field as a Bose-Einstein condensate? JCAP 11:034. https://doi.org/10.1088/1475-7516/2014/11/034. arXiv:1310.3319 [gr-qc]

Chambers KC, et al. (2016) The Pan-STARRS1 surveys. arXiv:1612.05560 [astro-ph.IM] (arXiv e-prints)

Chan JHH, Schive HY, Woo TP, Chiueh T (2018) How do stars affect $\psi$ DM haloes? MNRAS 478(2):26862699. https://doi.org/10.1093/mnras/sty900. arXiv:1712.01947 [astro-ph.GA]

Chandrasekhar S (1967) An introduction to the study of stellar structure. Dover, New York

Chapman S, Hoyos C, Oz Y (2014) Lifshitz superfluid hydrodynamics. JHEP 07:027. https://doi.org/10. 1007/JHEP07(2014)027. arXiv:1402.2981 [hep-th]

Chavanis P, Delfini L (2011) Mass-radius relation of Newtonian self-gravitating Bose-Einstein condensates with short-range interactions: II. Numerical results. Phys Rev D 84. https://doi.org/10.1103/ PhysRevD.84.043532. arXiv:1103.2054 [astro-ph.CO]

Chavanis PH (2011) Mass-radius relation of Newtonian self-gravitating Bose-Einstein condensates with short-range interactions: I. Analytical results. Phys Rev D 84:043531. https://doi.org/10.1103/ PhysRevD.84.043531. arXiv:1103.2050 [astro-ph.CO]

Chavanis PH (2012) Growth of perturbations in an expanding universe with Bose-Einstein condensate dark matter. Astron Astrophys 537:A127. https://doi.org/10.1051/0004-6361/201116905. arXiv:1103.2698 [astro-ph.CO]

Chavanis PH (2016) Collapse of a self-gravitating Bose-Einstein condensate with attractive self-interaction. Phys Rev D 94(8). https://doi.org/10.1103/PhysRevD.94.083007. arXiv:1604.05904 [astro-ph.CO]

Chavanis PH (2018) Phase transitions between dilute and dense axion stars. Phys Rev D 98(2):023009. https://doi.org/10.1103/PhysRevD.98.023009. arXiv:1710.06268 [gr-qc]

Chen SR, Schive HY, Chiueh T (2017) Jeans analysis for dwarf spheroidal galaxies in wave dark matter. MNRAS 468(2):1338-1348. https://doi.org/10.1093/mnras/stx449. arXiv:1606.09030 [astro-ph.GA]

Choi JR (2006) Interference in phase space of squeezed states for the time-dependent hamiltonian system. Int J Theor Phys 45(1):176-196. https://doi.org/10.1007/s10773-005-9016-9

Church BV, Mocz P, Ostriker JP (2019) Heating of Milky Way disc stars by dark matter fluctuations in cold dark matter and fuzzy dark matter paradigms. MNRAS 485(2):2861-2876. https://doi.org/10.1093/ mnras/stz534. arXiv:1809.04744 [astro-ph.GA]

Clowe D, Markevitch M, Bradac M, Gonzalez AH, Chung SM, Massey R, Zaritsky D (2012) On dark peaks and missing mass: a weak lensing mass reconstruction of the merging cluster system Abell 520. Astrophys J 758:128. https://doi.org/10.1088/0004-637X/758/2/128. arXiv:1209.2143 [astro-ph.CO]

Colin P, Avila-Reese V, Valenzuela O (2000) Substructure and halo density profiles in a warm dark matter cosmology. Astrophys J 542:622-630. https://doi.org/10.1086/317057. arXiv:astro-ph/0004115 
Connaughton C, Josserand C, Picozzi A, Pomeau Y, Rica S (2005) Condensation of classical nonlinear waves. Phys Rev Lett 95(26):263901. https://doi.org/10.1103/PhysRevLett.95.263901. arXiv:cond-mat/0502499 [cond-mat.stat-mech]

Conroy C, Wechsler RH, Kravtsov AV (2006) Modeling luminosity-dependent galaxy clustering through cosmic time. Astrophys J 647:201-214. https://doi.org/10.1086/503602. arXiv:astro-ph/0512234

Conti C (2012) Condensation dynamics: an enlightened daemon. Nat Phys 8(6):445-446. https://doi.org/ $10.1038 /$ nphys 2336

Cooper AP, Martínez-Delgado D, Helly J, Frenk C, Cole S, Crawford K, Zibetti S, Carballo-Bello JA, Gabany R (2011) The formation of shell galaxies similar to NGC 7600 in the cold dark matter cosmogony. Astrophys J Lett 743:L21. https://doi.org/10.1088/2041-8205/743/1/L21. arXiv:1111.2864 [astroph.GA]

Cooray A, Sheth RK (2002) Halo models of large scale structure. Phys Rep 372:1-129. https://doi.org/10. 1016/S0370-1573(02)00276-4. arXiv:astro-ph/0206508

Corasaniti P, Agarwal S, Marsh D, Das S (2017) Constraints on dark matter scenarios from measurements of the galaxy luminosity function at high redshifts. Phys Rev D 95(8):083512. https://doi.org/10.1103/ PhysRevD.95.083512. arXiv:1611.05892 [astro-ph.CO]

Côté S, Carignan C, Freeman KC (2000) The various kinematics of dwarf irregular galaxies in nearby groups and their dark matter distributions. Astron J 120(6):3027-3059. https://doi.org/10.1086/316883

Crain RA et al (2015) The EAGLE simulations of galaxy formation: calibration of subgrid physics and model variations. MNRAS 450(2):1937-1961. https://doi.org/10.1093/mnras/stv725. arXiv:1501.01311 [astro-ph.GA]

Dalal N, Kochanek CS (2002) Direct detection of CDM substructure. Astrophys J 572:25-33. https://doi. org/10.1086/340303. arXiv:astro-ph/0111456

Davidson S (2015) Axions: Bose-Einstein condensate or classical field? Astropart Phys 65:101-107. https:// doi.org/10.1016/j.astropartphys.2014.12.007. arXiv:1405.1139 [hep-ph]

Davidson S, Elmer M (2013) Bose-Einstein condensation of the classical axion field in cosmology? JCAP 12:034. https://doi.org/10.1088/1475-7516/2013/12/034. arXiv:1307.8024 [hep-ph]

Davis KB, Mewes MO, Andrews MR, van Druten NJ, Durfee DS, Kurn DM, Ketterle W (1995) BoseEinstein condensation in a gas of sodium atoms. Phys Rev Lett 75:3969-3973. https://doi.org/10. 1103/PhysRevLett.75.3969

Davoudiasl H, Denton PB (2019) Ultralight Boson dark matter and event horizon telescope observations of M87. Phys Rev Lett 123(2):021102. https://doi.org/10.1103/PhysRevLett.123.021102. arXiv:1904.09242 [astro-ph.CO]

Daylan T, Cyr-Racine FY, Diaz Rivero A, Dvorkin C, Finkbeiner DP (2018) Probing the small-scale structure in strongly lensed systems via transdimensional inference. Astrophys J 854(2):141. https://doi.org/10. 3847/1538-4357/aaaa1e. arXiv:1706.06111 [astro-ph.CO]

de Blok WJG, McGaugh SS, Bosma A, Rubin VC (2001a) Mass density profiles of LSB galaxies. Astrophys J Lett 552:L23-L26. https://doi.org/10.1086/320262. arXiv:astro-ph/0103102

de Blok WJG, McGaugh SS, Rubin VC (2001b) High-resolution rotation curves of low surface brightness galaxies. II. Mass models. Astron J 122(5):2396-2427. https://doi.org/10.1086/323450

de Boer TJL, Erkal D, Gieles M (2020) A closer look at the spur, blob, wiggle, and gaps in GD-1. MNRAS 494(4):5315-5332. https://doi.org/10.1093/mnras/staa917. arXiv:1911.05745 [astro-ph.GA]

DeBoer DR et al (2017) Hydrogen epoch of reionization array (HERA). Publ Astron Soc Pac 129(974):045001. https://doi.org/10.1088/1538-3873/129/974/045001. arXiv:1606.07473 [astroph.IM]

Dekel A, Silk J (1986) The origin of dwarf galaxies, cold dark matter, and biased galaxy formation. Astrophys J 303:39-55. https://doi.org/10.1086/164050

Del Popolo A, Le Delliou M (2017) Small scale problems of the $\Lambda$ CDM model: a short review. Galaxies 5(1):17. https://doi.org/10.3390/galaxies5010017. arXiv:1606.07790 [astro-ph.CO]

Despali G, Vegetti S, White SDM, Giocoli C, van den Bosch FC (2018) Modelling the line-of-sight contribution in substructure lensing. MNRAS 475(4):5424-5442. https://doi.org/10.1093/mnras/sty159. arXiv:1710.05029 [astro-ph.CO]

Dev PSB, Lindner M, Ohmer S (2017) Gravitational waves as a new probe of Bose-Einstein condensate dark matter. Phys Lett B 773:219-224. https://doi.org/10.1016/j.physletb.2017.08.043. arXiv:1609.03939 [hep-ph]

Di Luzio L, Giannotti M, Nardi E, Visinelli L (2020) The landscape of QCD axion models. Phys Rep 870:1-117. https://doi.org/10.1016/j.physrep.2020.06.002. arXiv:2003.01100 [hep-ph] 
Dimonte D, Falconi M, Olgiati A (2018) On some rigorous aspects of fragmented condensation. arXiv:1809.03586. arXiv:1809.03586 [cond-mat.quant-gas] (arXiv e-prints)

Dine M, Fischler W (1983) The not so harmless axion. Phys Lett B 120:137-141. https://doi.org/10.1016/ 0370-2693(83)90639-1

Dolan SR (2013) Superradiant instabilities of rotating black holes in the time domain. Phys Rev D 87(12):124026. https://doi.org/10.1103/PhysRevD.87.124026. arXiv:1212.1477 [gr-qc]

Drlica-Wagner A et al (2015) Eight ultra-faint galaxy candidates discovered in year two of the dark energy survey. Astrophys J 813(2):109. https://doi.org/10.1088/0004-637X/813/2/109. arXiv:1508.03622 [astro-ph.GA]

Drlica-Wagner A, et al. (2019) Probing the fundamental nature of dark matter with the large synoptic survey telescope. arXiv:1902.01055 [astro-ph.CO] (arXiv e-prints)

Du X, Behrens C, Niemeyer JC (2017a) Substructure of fuzzy dark matter haloes. MNRAS 465(1):941-951. https://doi.org/10.1093/mnras/stw2724. arXiv:1608.02575 [astro-ph.CO]

Du X, Behrens C, Niemeyer JC, Schwabe B (2017b) Core-halo mass relation of ultralight axion dark matter from merger history. Phys Rev D 95:043519. https://doi.org/10.1103/PhysRevD.95.043519

Du XL (2018) Structure formation with ultralight axion dark matter. PhD thesis, University of Göttingen. http://hdl.handle.net/21.11130/00-1735-0000-0003-C1C3-6

Du X, Schwabe B, Niemeyer JC, Bürger D (2018) Tidal disruption of fuzzy dark matter subhalo cores. Phys Rev D 97(6):063507. https://doi.org/10.1103/PhysRevD.97.063507.arXiv:1801.04864 [astro-ph.GA]

Dubovsky S, Gregoire T, Nicolis A, Rattazzi R (2006) Null energy condition and superluminal propagation. JHEP 03:025. https://doi.org/10.1088/1126-6708/2006/03/025. arXiv:hep-th/0512260

Dubovsky S, Hui L, Nicolis A, Son DT (2012) Effective field theory for hydrodynamics: thermodynamics, and the derivative expansion. Phys Rev D 85:085029. https://doi.org/10.1103/PhysRevD.85.085029. arXiv:1107.0731 [hep-th]

Dutton AA, Macciò AV, Obreja A, Buck T (2019) NIHAO-XVIII. Origin of the MOND phenomenology of galactic rotation curves in a $\Lambda$ CDM universe. MNRAS 485(2):1886-1899. https://doi.org/10.1093/ mnras/stz531. arXiv:1902.06751 [astro-ph.GA]

Dvali G, Zell S (2018) Classicality and quantum break-time for cosmic axions. JCAP 07:064. https://doi. org/10.1088/1475-7516/2018/07/064. arXiv:1710.00835 [hep-ph]

Dwornik M, Keresztes Z, Gergely L (2014) Rotation curves in Bose-Einstein condensate dark matter halos. In: Kinjo N, Nakajima A (eds) Recent development in dark matter research. Nova Science, New York, pp 195-219 arXiv:1312.3715 [gr-qc]

Dyachenko S, Newell AC, Pushkarev A, Zakharov VE (1992) Optical turbulence: weak turbulence, condensates and collapsing filaments in the nonlinear Schrödinger equation. Phys D 57(1-2):96-160. https:// doi.org/10.1016/0167-2789(92)90090-A

East WE, Pretorius F (2017) Superradiant instability and backreaction of massive vector fields around Kerr black holes. Phys Rev Lett 119(4):041101. https://doi.org/10.1103/PhysRevLett.119.041101. arXiv:1704.04791 [gr-qc]

Eastwood MW et al (2019) The $21 \mathrm{~cm}$ power spectrum from the cosmic dawn: first results from the OVRO-LWA. Astron J 158(2):84. https://doi.org/10.3847/1538-3881/ab2629. arXiv:1906.08943 [astro-ph.CO]

Eby J, Suranyi P, Vaz C, Wijewardhana L (2015) Axion stars in the infrared limit. JHEP 03:080. doi: https:// doi.org/10.1007/JHEP11(2016)134, [Erratum: JHEP 11, 134 (2016)]. arXiv:1412.3430 [hep-th]

Eby J, Kouvaris C, Nielsen NG, Wijewardhana L (2016a) Boson stars from self-interacting dark matter. JHEP 02:028. https://doi.org/10.1007/JHEP02(2016)028. arXiv:1511.04474 [hep-ph]

Eby J, Suranyi P, Wijewardhana L (2016b) The lifetime of axion stars. Mod Phys Lett A 31(15):1650090. https://doi.org/10.1142/S0217732316500905. arXiv:1512.01709 [hep-ph]

Eby J, Leembruggen M, Street L, Suranyi P, Wijewardhana LCR (2018) Approximation methods in the study of boson stars. Phys Rev D 98(12):123013. https://doi.org/10.1103/PhysRevD.98.123013. arXiv:1809.08598 [hep-ph]

Eby J, Leembruggen M, Street L, Suranyi P, Wijewardhana LR (2019) Global view of QCD axion stars. Phys Rev D 100(6):063002. https://doi.org/10.1103/PhysRevD.100.063002. arXiv:1905.00981 [hep-ph]

Edwards F, Kendall E, Hotchkiss S, Easther R (2018) PyUltraLight: a pseudo-spectral solver for ultralight dark matter dynamics. JCAP 10:027. https://doi.org/10.1088/1475-7516/2018/10/027. arXiv:1807.04037 [astro-ph.CO]

Efstathiou G (1992) Suppressing the formation of dwarf galaxies via photoionization. MNRAS 256:43P$47 \mathrm{P}$ 
Efstathiou G, Davis M, White SDM, Frenk CS (1985) Numerical techniques for large cosmological $N$-body simulations. Astrophys J Suppl 57:241-260. https://doi.org/10.1086/191003

Einasto $\mathrm{J}$ (1965) On the construction of a composite model for the galaxy and on the determination of the system of galactic parameters. Trudy Inst Astrofiz Alma-Ata 5:87-100 (In Russian)

Eisenstein DJ, Hu W (1998) Baryonic features in the matter transfer function. Astrophys J 496:605. https:// doi.org/10.1086/305424. arXiv:astro-ph/9709112

El-Zant A, Freundlich J, Combes F (2016) From cusps to cores: a stochastic model. MNRAS 461(2):17451759. https://doi.org/10.1093/mnras/stw1398. arXiv:1603.00526 [astro-ph.GA]

El-Zant A, Roupas Z, Silk J (2020a) Ejection of supermassive black holes and implications for merger rates in fuzzy dark matter haloes. arXiv:2009.10167 [astro-ph.GA] (arXiv e-prints)

El-Zant AA, Freundlich J, Combes F, Halle A (2020b) The effect of fluctuating fuzzy axion haloes on stellar dynamics: a stochastic model. MNRAS 492(1):877-894. https://doi.org/10.1093/mnras/stz3478. arXiv:1908.09061 [astro-ph.GA]

Erkal D, Belokurov V (2015) Forensics of subhalo-stream encounters: the three phases of gap growth. MNRAS 450(1):1136-1149. https://doi.org/10.1093/mnras/stv655. arXiv:1412.6035 [astro-ph.GA]

Erkal D, Belokurov V, Bovy J, Sanders JL (2016) The number and size of subhalo-induced gaps in stellar streams. MNRAS 463(1):102-119. https://doi.org/10.1093/mnras/stw1957. arXiv:1606.04946 [astroph.GA]

Erken O, Sikivie P, Tam H, Yang Q (2012) Cosmic axion thermalization. Phys Rev D 85:063520. https:// doi.org/10.1103/PhysRevD.85.063520. arXiv:1111.1157 [astro-ph.CO]

Fairbairn M, Marsh DJE, Quevillon J, Rozier S (2018) Structure formation and microlensing with axion miniclusters. Phys Rev D 97(8):083502. https://doi.org/10.1103/PhysRevD.97.083502. arXiv:1707.03310 [astro-ph.CO]

Famaey B, McGaugh S (2012) Modified Newtonian Dynamics (MOND): observational phenomenology and relativistic extensions. Living Rev Relativ 15:10. https://doi.org/10.12942/lrr-2012-10. arXiv:1112.3960 [astro-ph.CO]

Fan J (2016) Ultralight repulsive dark matter and BEC. Phys Dark Univ 14:84-94. https://doi.org/10.1016/ j.dark.2016.10.005. arXiv:1603.06580 [hep-ph]

Fernandes RM, Chubukov AV (2017) Low-energy microscopic models for iron-based superconductors: a review. Rep Progr Phys 80(1):014503. https://doi.org/10.1088/1361-6633/80/1/014503. arXiv:1607.00865 [cond-mat.str-el]

Ferreira EGM (2021) Unified Superfluid Dark Sector. In: Paranjape MB, MacKenzie R, Thomova Z, Winternitz P, Witczak-Krempa W (eds) Quantum Theory and Symmetries. CRM Series in Mathematical Physics. Springer, Cham. https://doi.org/10.1007/978-3-030-55777-5_40. arXiv:1911.07371 [astroph.CO]

Ferreira EGM, Franzmann G, Khoury J, Brandenberger R (2019) Unified superfluid dark sector. JCAP 08:027. https://doi.org/10.1088/1475-7516/2019/08/027. arXiv:1810.09474 [astro-ph.CO]

Ferrero I, Navarro JF, Abadi MG, Sales LV, Bower RG, Crain RA, Frenk CS, Schaller M, Schaye J, Theuns T (2017) Size matters: abundance matching, galaxy sizes, and the Tully-Fisher relation in EAGLE. Mon Not R Astron Soc 464(4):4736-4746. https://doi.org/10.1093/mnras/stw2691. arXiv: 1607.03100 [astro-ph.GA]

Fetter AL (2009) Rotating trapped Bose-Einstein condensates. Rev Mod Phys 81:647-691. https://doi.org/ 10.1103/RevModPhys.81.647

Feynman RP (1955) Application of quantum mechanics to liquid helium. In: Gorter CJ (ed) Progress in low temperature physics. Interscience, New York, pp 17-53. https://doi.org/10.1016/S00796417(08)60077-3

Fitts A et al (2017) FIRE in the field: simulating the threshold of galaxy formation. MNRAS 471(3):35473562. https://doi.org/10.1093/mnras/stx1757. arXiv:1611.02281 [astro-ph.GA]

Flores RA, Primack JR (1994) Observational and theoretical constraints on singular dark matter halos. Astrophys J Lett 427:L1-4. https://doi.org/10.1086/187350. arXiv:astro-ph/9402004

Frieman JA, Hill CT, Stebbins A, Waga I (1995) Cosmology with ultralight pseudo NambuGoldstone bosons. Phys Rev Lett 75:2077-2080. https://doi.org/10.1103/PhysRevLett.75.2077. arXiv:astro-ph/9505060

Gaia Collaboration (2016) The Gaia mission. Astron Astrophys 595:A1. https://doi.org/10.1051/00046361/201629272. arXiv:1609.04153 [astro-ph.IM] 
Gao L, Navarro JF, Cole S, Frenk C, White SDM, Springel V, Jenkins A, Neto AF (2008) The redshift dependence of the structure of massive LCDM halos. MNRAS 387:536. https://doi.org/10.1111/j. 1365-2966.2008.13277.x. arXiv:0711.0746 [astro-ph]

Garaldi E, Romano-Díaz E, Borzyszkowski M, Porciani C (2017) ZOMG-III. The effect of halo assembly on the satellite population. MNRAS 473(2):2234-2250. https://doi.org/10.1093/mnras/stx2489. arXiv:1707.01108 [astro-ph.GA]

Garaldi E, Romano-Díaz E, Porciani C, Pawlowski MS (2018) Radial acceleration relation of $\Lambda$ CDM satellite galaxies. Phys Rev Lett 120(26):261301. https://doi.org/10.1103/PhysRevLett.120.261301. arXiv: 1712.04448 [astro-ph.GA]

Garny M, Konstandin T, Rubira H (2020) The Schrödinger-Poisson method for large-scale structure. JCAP 04:003. https://doi.org/10.1088/1475-7516/2020/04/003. arXiv:1911.04505 [astro-ph.CO]

Garrison-Kimmel S, Boylan-Kolchin M, Bullock JS, Kirby EN (2014) Too big to fail in the local group. MNRAS 444(1):222-236. https://doi.org/10.1093/mnras/stu1477. arXiv:1404.5313 [astro-ph.GA]

Gell-Mann M (1962) Symmetries of baryons and mesons. Phys Rev 125:1067-1084. https://doi.org/10. 1103/PhysRev.125.1067

Gell-Mann M, Oakes RJ, Renner B (1968) Behavior of current divergences under $\mathrm{SU}_{3} \times \mathrm{SU}_{3}$. Phys Rev 175:2195-2199. https://doi.org/10.1103/PhysRev.175.2195

Gentile G, Burkert A, Salucci P, Klein U, Walter F (2005) The dwarf galaxy DDO 47 as a dark matter laboratory: testing cusps hiding in triaxial halos. Astrophys J Lett 634:L145-L148. https://doi.org/10. 1086/498939. arXiv:astro-ph/0506538

Gentile G, Salucci P, Klein U, Granato GL (2007) NGC 3741: dark halo profile from the most extended rotation curve. Mon Not R Astron Soc 375:199-212. https://doi.org/10.1111/j.1365-2966.2006.11283. X. arXiv:astro-ph/0611355

Goldstone J (1961) Field theories with superconductor solutions. Nuovo Cim 19:154-164. https://doi.org/ 10.1007/BF02812722

Goldstone J, Salam A, Weinberg S (1962) Broken symmetries. Phys Rev 127:965-970. https://doi.org/10. 1103/PhysRev.127.965

González JA, Guzmán FS (2011) Interference pattern in the collision of structures in the BEC dark matter model: comparison with fluids. Phys Rev D 83:103513. https://doi.org/10.1103/PhysRevD.83.103513. arXiv:1105.2066 [astro-ph.CO]

González-Morales AX, Marsh DJ, Peñarrubia J, Ureña López LA (2017) Unbiased constraints on ultralight axion mass from dwarf spheroidal galaxies. MNRAS 472(2):1346-1360. https://doi.org/10.1093/ mnras/stx 1941. arXiv:1609.05856 [astro-ph.CO]

Goodman J (2000) Repulsive dark matter. New Astron 5:103. https://doi.org/10.1016/S13841076(00)00015-4. arXiv:astro-ph/0003018

Governato F, Brook C, Mayer L, Brooks A, Rhee G, Wadsley J, Jonsson P, Willman B, Stinson G, Quinn T, Madau P (2010) Bulgeless dwarf galaxies and dark matter cores from supernova-driven outflows. Nature 463(7278):203-206. https://doi.org/10.1038/nature08640. arXiv:0911.2237 [astro-ph.CO]

Governato F, Zolotov A, Pontzen A, Christensen C, Oh SH, Brooks AM, Quinn T, Shen S, Wadsley J (2012) Cuspy no more: how outflows affect the central dark matter and baryon distribution in $\Lambda$ cold dark matter galaxies. MNRAS 422:1231-1240. https://doi.org/10.1111/j.1365-2966.2012.20696.x. arXiv:1202.0554 [astro-ph.CO]

Graham PW, Irastorza IG, Lamoreaux SK, Lindner A, van Bibber KA (2015a) Experimental searches for the axion and axion-like particles. Annu Rev Nucl Part Sci 65:485-514. https://doi.org/10.1146/annurevnucl-102014-022120. arXiv:1602.00039 [hep-ex]

Greiter M, Wilczek F, Witten E (1989) Hydrodynamic relations in superconductivity. Mod Phys Lett B 3:903. https://doi.org/10.1142/S0217984989001400

Grillmair CJ, Dionatos O (2006) Detection of a 63 degree cold stellar stream in the Sloan digital sky survey. Astrophys J Lett 643:L17-L20. https://doi.org/10.1086/505111. arXiv:astro-ph/0604332

Grin D, Amin MA, Gluscevic V, Hložek R, Marsh DJ, Poulin V, Prescod-Weinstein C, Smith TL (2019) Gravitational probes of ultra-light axions. arXiv:1904.09003 [astro-ph.CO] (arXiv e-prints)

Guadagnini E (2017) Critical angular velocity for vortex lines formation. J Stat Mech 1707(7):073104. https://doi.org/10.1088/1742-5468/aa7864. arXiv:1706.04831 [cond-mat.other]

Guth AH, Hertzberg MP, Prescod-Weinstein C (2015) Do dark matter axions form a condensate with long-range correlation? Phys Rev D 92(10):103513. https://doi.org/10.1103/PhysRevD.92.103513. arXiv:1412.5930 [astro-ph.CO] 
Guzmán FS, Ureña-López LA (2004) Evolution of the Schrödinger-Newton system for a selfgravitating scalar field. Phys Rev D 69:124033. https://doi.org/10.1103/PhysRevD.69.124033. arXiv:gr-qc/0404014

Guzmán FS, Ureña-López LA (2006) Gravitational cooling of self-gravitating Bose-condensates. Astrophys J 645:814-819. https://doi.org/10.1086/504508. arXiv:astro-ph/0603613

Guzmán FS, Matos T, Villegas HB (1999) Scalar fields as dark matter in spiral galaxies: comparison with experiments. Astron Nachr 320(3):97. https://doi.org/10.1002/1521-3994(199907)320:3<97::AIDASNA97>3.0.CO;2-M

Guzmán FS, Lora-Clavijo FD, González-Avilés JJ, Rivera-Paleo FJ (2014) Rotation curves of rotating Galactic Bose-Einstein condensate dark matter halos. Phys Rev D 89(6):063507. https://doi.org/10. 1103/PhysRevD.89.063507. arXiv:1310.3909 [astro-ph.CO]

Hamner C, Chang JJ, Engels P, Hoefer MA (2011) Generation of dark-bright soliton trains in superfluidsuperfluid counterflow. Phys Rev Lett 106(6):065302. https://doi.org/10.1103/PhysRevLett.106. 065302. arXiv:1005.2610 [cond-mat.quant-gas]

Hannuksela OA, Wong KWK, Brito R, Berti E, Li TGF (2019) Probing the existence of ultralight bosons with a single gravitational-wave measurement. Nat Astron 3(5):447-451. https://doi.org/10.1038/s41550019-0712-4. arXiv:1804.09659 [astro-ph.HE]

Harko T (2011) Bose-Einstein condensation of dark matter solves the core/cusp problem. JCAP 05:022. https://doi.org/10.1088/1475-7516/2011/05/022. arXiv:1105.2996 [astro-ph.CO]

Harko T (2014) Gravitational collapse of Bose-Einstein condensate dark matter halos. Phys Rev D 89(8):084040. https://doi.org/10.1103/PhysRevD.89.084040. arXiv:1403.3358 [gr-qc]

Harko T, Lake MJ (2015) Bose-Einstein condensate strings. Phys Rev D 91:045012. https://doi.org/10. 1103/PhysRevD.91.045012. arXiv:1410.6899 [gr-qc]

Harrison R, Moroz I, Tod KP (2002) A numerical study of the Schrödinger-Newton equation 1: Perturbing the spherically-symmetric stationary states. arXiv:math-ph/0208045 [math-ph] (arXiv e-prints)

Hartman STH, Winther HA, Mota DF (2019) Collapse of spherical overdensities in superfluid models of dark matter. arXiv:1911.11498 [astro-ph.CO] (arXiv e-prints)

Harvey D, Massey R, Kitching T, Taylor A, Tittley E (2015) The non-gravitational interactions of dark matter in colliding galaxy clusters. Science 347:1462-1465. https://doi.org/10.1126/science.1261381. arXiv:1503.07675 [astro-ph.CO]

Hayashi K, Obata I (2020) Non-sphericity of ultralight axion dark matter haloes in the Galactic dwarf spheroidal galaxies. MNRAS 491(1):615-633. https://doi.org/10.1093/mnras/stz2950. arXiv:1902.03054 [astro-ph.CO]

Hayashi K, Chiba M, Ishiyama T (2020) Diversity of dark matter density profiles in the galactic dwarf spheroidal satellites. Astrophys J 904(1):45. https://doi.org/10.3847/1538-4357/abbe0a. arXiv:2007.13780 [astro-ph.GA]

Hayashi K, Ferreira EGM, Chan HYJ (2021) Narrowing the mass range of fuzzy dark matter with ultra-faint dwarfs. Astrophys J Lett 912(1):L3. https://doi.org/10.3847/2041-8213/abf501. arXiv:2102.05300 [astro-ph.CO]

Hezaveh YD et al (2016) Detection of lensing substructure using ALMA observations of the dusty galaxy SDP8.1. Astrophys J 823(1):37. https://doi.org/10.3847/0004-637X/823/1/37. arXiv:1601.01388 [astro-ph.CO]

Hiramatsu T, Kawasaki M, Saikawa K, Sekiguchi T (2012) Production of dark matter axions from collapse of string-wall systems. Phys Rev D. https://doi.org/10.1103/PhysRevD.85.105020. [Erratum: Phys. Rev. D 86, 089902 (2012)] arXiv:1202.5851 [hep-ph]

Hložek R, Grin D, Marsh DJE, Ferreira PG (2015) A search for ultralight axions using precision cosmological data. Phys Rev D 91(10):103512. https://doi.org/10.1103/PhysRevD.91.103512. arXiv:1410.2896 [astro-ph.CO]

Hložek R, Marsh DJE, Grin D, Allison R, Dunkley J, Calabrese E (2017) Future CMB tests of dark matter: ultralight axions and massive neutrinos. Phys Rev D 95:123511. https://doi.org/10.1103/PhysRevD. 95.123511

Hložek R, Marsh DJE, Grin D (2018) Using the full power of the cosmic microwave background to probe axion dark matter. MNRAS 476(3):3063-3085. https://doi.org/10.1093/mnras/sty271. arXiv:1708.05681 [astro-ph.CO]

Hodson A, Zhao H, Khoury J, Famaey B (2017) Galaxy clusters in the context of superfluid dark matter. Astron Astrophys 607:A108. https://doi.org/10.1051/0004-6361/201630069. arXiv:1611.05876 [astro-ph.CO] 
Hofmann CP (1999) Spin wave scattering in the effective Lagrangian perspective. Phys Rev B 60:388. https://doi.org/10.1103/PhysRevB.60.388. arXiv:cond-mat/9805277

Hossenfelder S, Mistele T (2019) Strong lensing with superfluid dark matter. JCAP 02:001. https://doi.org/ 10.1088/1475-7516/2019/02/001. arXiv:1809.00840 [astro-ph.GA]

Hossenfelder S, Mistele T (2020) The Milky Way's rotation curve with superfluid dark matter. MNRAS 498(3):3484-3491. https://doi.org/10.1093/mnras/staa2594 arXiv:2003.07324 [astro-ph.GA]

Hsueh JW, Oldham L, Spingola C, Vegetti S, Fassnacht CD, Auger MW, Koopmans LVE, McKean JP, Lagattuta DJ (2017) SHARP-IV. An apparent flux-ratio anomaly resolved by the edge-on disc in B0712+472. Mon Not R Astron Soc 469(3):3713-3721. https://doi.org/10.1093/mnras/stx1082. arXiv:1701.06575 [astro-ph.GA]

Hu W, Barkana R, Gruzinov A (2000a) Cold and fuzzy dark matter. Phys Rev Lett 85:1158-1161. https:// doi.org/10.1103/PhysRevLett.85.1158. arXiv:astro-ph/0003365

Hu W, Barkana R, Gruzinov A (2000b) Fuzzy cold dark matter: the wave properties of ultralight particles. Phys Rev Lett 85:1158-1161. https://doi.org/10.1103/PhysRevLett.85.1158

Hui L, Ostriker JP, Tremaine S, Witten E (2017) Ultralight scalars as cosmological dark matter. Phys Rev D 95(4):043541. https://doi.org/10.1103/PhysRevD.95.043541. arXiv:1610.08297 [astro-ph.CO]

Hui L, Joyce A, Landry MJ, Li X (2020) Vortices and waves in light dark matter. JCAP 01(2021):011. https://doi.org/10.1088/1475-7516/2021/01/011 arXiv:2004.01188 [astro-ph.CO]

Hwang J, Noh H (2009) Axion as a cold dark matter candidate. Phys Lett B 680:1-3. https://doi.org/10. 1016/j.physletb.2009.08.031. arXiv:0902.4738 [astro-ph.CO]

Ianeselli S, Menotti C, Smerzi A (2006) Beyond the Landau criterion for superfluidity. J Phys B 39(10):S135S142. https://doi.org/10.1088/0953-4075/39/10/S13. arXiv:cond-mat/0509670 [cond-mat.other]

Ibata RA, Lewis GF, Irwin MJ (2002) Uncovering CDM halo substructure with tidal streams. MNRAS 332:915. https://doi.org/10.1046/j.1365-8711.2002.05358.x. arXiv:astro-ph/0110690

Ibata R, Thomas G, Famaey B, Malhan K, Martin N, Monari G (2020) Detection of strong epicyclic density spikes in the GD-1 stellar stream: an absence of evidence for the influence of dark matter subhalos? Astrophys J 891(2):161. https://doi.org/10.3847/1538-4357/ab7303. arXiv:2002.01488 [astro-ph.GA]

Illustris (2014) Illustris. http://www.illustris-project.org

Iršič V, Viel M, Haehnelt MG, Bolton JS, Becker GD (2017) First constraints on fuzzy dark matter from Lyman- $\alpha$ forest data and hydrodynamical simulations. Phys Rev Lett 119(3):031302. https://doi.org/ 10.1103/PhysRevLett.119.031302. arXiv:1703.04683 [astro-ph.CO]

Itzykson C, Zuber J (1980) Quantum field theory. International series in pure and applied physics, McGrawHill, New York

Ivezić Ž (2019) LSST: from science drivers to reference design and anticipated data products. Astrophys J 873(2):111. https://doi.org/10.3847/1538-4357/ab042c. arXiv:0805.2366 [astro-ph]

Jacobs DM, Starkman GD, Lynn BW (2015) Macro dark matter. MNRAS 450(4):3418-3430. https://doi. org/10.1093/mnras/stv774. arXiv:1410.2236 [astro-ph.CO]

Jee MJ, Mahdavi A, Hoekstra H, Babul A, Dalcanton JJ, Carroll P, Capak P (2012) A study of the dark core in A520 with Hubble Space Telescope: the mystery deepens. Astrophys J 747:96. https://doi.org/10. 1088/0004-637X/747/2/96. arXiv:1202.6368 [astro-ph.CO]

Jee MJ, Hoekstra H, Mahdavi A, Babul A (2014) Hubble Space Telescope/Advanced Camera for Surveys confirmation of the dark substructure in A520. Astrophys J 783:78. https://doi.org/10.1088/0004637X/783/2/78. arXiv:1401.3356 [astro-ph.CO]

Jetzer P (1992) Boson stars. Phys Rep 220:163-227. https://doi.org/10.1016/0370-1573(92)90123-H

Ji SU, Sin SJ (1994) Late time phase transition and the galactic halo as a Bose liquid: 2. The effect of visible matter. Phys Rev D 50:3655-3659. https://doi.org/10.1103/PhysRevD.50.3655. arXiv:hep-ph/9409267

Jing YP, Suto Y (2002) Triaxial modeling of halo density profiles with high-resolution N-body simulations. Astrophys J 574(2):538-553. https://doi.org/10.1086/341065. arXiv:astro-ph/0202064 [astro-ph]

Joachimi B et al (2015) Galaxy alignments: an overview. Space Sci Rev 193(1-4):1-65. https://doi.org/10. 1007/s11214-015-0177-4. arXiv:1504.05456 [astro-ph.GA]

Johnston KV, Spergel DN, Haydn C (2002) How lumpy is the Milky Way's dark matter halo? Astrophys J 570:656. https://doi.org/10.1086/339791. arXiv:astro-ph/0111196

Josephson BD (1962) Possible new effects in superconductive tunnelling. Phys Lett 1:251-253. https://doi. org/10.1016/0031-9163(62)91369-0 
Kain B, Ling HY (2010) Vortices in Bose-Einstein condensate dark matter. Phys Rev D 82:064042. https:// doi.org/10.1103/PhysRevD.82.064042. arXiv:1004.4692 [hep-ph]

Kaloper N, Sorbo L (2006) Of PNGB quintessence. JCAP 04:007. https://doi.org/10.1088/1475-7516/ 2006/04/007. arXiv:astro-ph/0511543

Kapitza P (1938) Viscosity of liquid helium below the $\lambda$-point. Nature 141(3558):74. https://doi.org/10. 1038/141074a0

Kasun SF, Evrard AE (2005) Shapes and alignments of galaxy cluster halos. Astrophys J 629(2):781-790. https://doi.org/10.1086/430811. arXiv:astro-ph/0408056 [astro-ph]

Kaup DJ (1968) Klein-Gordon Geon. Phys Rev 172:1331-1342. https://doi.org/10.1103/PhysRev.172.1331

Kawasaki M, Nakayama K (2013) Axions: theory and cosmological role. Annu Rev Nucl Part Sci 63:69-95. https://doi.org/10.1146/annurev-nucl-102212-170536. arXiv:1301.1123 [hep-ph]

Kendall E, Easther R (2020) The core-cusp problem revisited: ULDM vs. CDM. Publ Astron Soc Austral 37:e009. https://doi.org/10.1017/pasa.2020.3. arXiv:1908.02508 [astro-ph.CO]

Kevrekidis PG, Rasmussen KØ, Bishop AR (2001) The discrete nonlinear Schrödinger equation. Int J Mod Phys B 15(21):2833-2900. https://doi.org/10.1142/S0217979201007105

Khlebnikov S (2000) Short scale gravitational instability in a disordered Bose gas. Phys Rev D 62:043519. https://doi.org/10.1103/PhysRevD.62.043519. arXiv:astro-ph/9911218

Khlopov MY (2019) Direct and indirect probes for composite dark matter. Front Phys 7:4. https://doi.org/ 10.3389/fphy.2019.00004

Khlopov MY, Malomed BA, Zeldovich YB (1985) Gravitational instability of scalar fields and formation of primordial black holes. MNRAS 215:575-589. https://doi.org/10.1093/mnras/215.4.575

Khoury J (2016) Another path for the emergence of modified galactic dynamics from dark matter superfluidity. Phys Rev D 93(10):103533. https://doi.org/10.1103/PhysRevD.93.103533. arXiv:1602.05961 [astro-ph.CO]

Kiessling A et al. (2015) Galaxy alignments: theory, modelling and simulations. Space Sci Rev 193(14), 67-136. https://doi.org/10.1007/s11214-015-0203-6, [Erratum: Space Sci Rev 193, 137 (2015)]. arXiv: 1504.05546 [astro-ph.GA]

Kim JE, Carosi G (2010) Axions and the strong CP problem. Rev Mod Phys 82:557-602. https://doi.org/10. 1103/RevModPhys.82.557 [Erratum: Rev Mod Phys 91, 049902 (2019)]. arXiv:0807.3125 [hep-ph]

Kirkpatrick K, Mirasola AE, Prescod-Weinstein C (2020) Relaxation times for Bose-Einstein condensation in axion miniclusters. Phys Rev D 102(10):103012. https://doi.org/10.1103/PhysRevD.102.103012. arXiv:2007.07438 [hep-ph]

Kobayashi T, Murgia R, De Simone A, Iršič V, Viel M (2017) Lyman- $\alpha$ constraints on ultralight scalar dark matter: implications for the early and late universe. Phys Rev D 96(12):123514. https://doi.org/10. 1103/PhysRevD.96.123514. arXiv:1708.00015 [astro-ph.CO]

Koekemoer AM, Avila RJ, Hammer D, Mack J, Ogaz S, Anderson J, Barker EA, Hilbert B, Gonzaga S, Grogin NA, Fruchter AS, Lotz J, Lucas RA, Mountain M, Sokol J (2014) The HST frontier fields: science data pipeline, products, and first data release. In: American Astronomical Society Meeting Abstracts \#223, p 254.02

Kolb EW, Tkachev II (1993) Axion miniclusters and Bose stars. Phys Rev Lett 71:3051-3054. https://doi. org/10.1103/PhysRevLett.71.3051. arXiv:hep-ph/9303313

Kolb EW, Tkachev II (1994) Nonlinear axion dynamics and formation of cosmological pseudosolitons. Phys Rev D 49:5040-5051. https://doi.org/10.1103/PhysRevD.49.5040. arXiv:astro-ph/9311037

Kolb EW, Turner MS (1990) The early universe, Frontiers in Physics, vol 69. Addison-Wesley, Redwood City

Kolmakov GV, McClintock PVE, Nazarenko SV (2014) Wave turbulence in quantum fluids. Proc Natl Acad Sci USA 111(Supplement 1):4727-4734. https://doi.org/10.1073/pnas.1312575110

Kolmogorov AN (1933) Grundbegriffe der Wahrscheinlichkeitsrechnung. Springer, Berlin. https://doi.org/ 10.1007/978-3-642-49888-6 (2nd English edn., Foundations of Probability (1950), published by Chelsea, New York)

Koopmans LVE (2005) Gravitational-mass imaging of CDM substructure. Mon Not R Astron Soc 363:1136. https://doi.org/10.1111/j.1365-2966.2005.09523.x. arXiv:astro-ph/0501324

Koopmans LVE et al (2015) The cosmic dawn and epoch of reionization with the Square Kilometre Array. PoS AASKA 14:001. https://doi.org/10.22323/1.215.0001. arXiv:1505.07568 [astro-ph.CO]

Kravtsov AV, Berlind AA, Wechsler RH, Klypin AA, Gottloeber S, Allgood B, Primack JR (2004) The dark side of the halo occupation distribution. Astrophys J 609:35-49. https://doi.org/10.1086/420959. arXiv:astro-ph/0308519 
Kuhlen M, Vogelsberger M, Angulo R (2012) Numerical simulations of the dark universe: state of the art and the next decade. Phys Dark Univ 1:50-93. https://doi.org/10.1016/j.dark.2012.10.002. arXiv:1209.5745 [astro-ph.CO]

Lage C, Farrar GR (2015) The bullet cluster is not a cosmological anomaly. JCAP 02:038. https://doi.org/ 10.1088/1475-7516/2015/02/038. arXiv:1406.6703 [astro-ph.GA]

Lancaster L, Giovanetti C, Mocz P, Kahn Y, Lisanti M, Spergel DN (2020) Dynamical friction in a fuzzy dark matter universe. JCAP 01:001. https://doi.org/10.1088/1475-7516/2020/01/001. arXiv:1909.06381 [astro-ph.CO]

Landau LD, Lifshitz EM (1980) Statistical physics, vol 5. Elsevier, New York

Laporte CFP, Johnston KV, Gómez FA, Garavito-Camargo N, Besla G (2018) The influence of Sagittarius and the Large Magellanic Cloud on the stellar disc of the Milky Way Galaxy. MNRAS 481(1):286-306. https://doi.org/10.1093/mnras/sty1574. arXiv:1710.02538 [astro-ph.GA]

Lazar A, Bullock JS, Boylan-Kolchin M, Chan TK, Hopkins PF, Graus AS, Wetzel A, El-Badry K, Wheeler C, Straight MC, Kereš D, Faucher-Giguère CA, Fitts A, Garrison-Kimmel S (2020) A dark matter profile to model diverse feedback-induced core sizes of $\Lambda$ CDM haloes. MNRAS 497(2):2393-2417. https://doi.org/10.1093/mnras/staa2101. arXiv:2004.10817 [astro-ph.GA]

Lee JW (2009) Are galaxies extending? Phys Lett B 681:118-121. https://doi.org/10.1016/j.physletb.2009. 10.005. arXiv:0805.2877 [astro-ph]

Lee JW (2018) Brief history of ultra-light scalar dark matter models. EPJ Web Conf 168:06005. https://doi. org/10.1051/epjconf/201816806005. arXiv:1704.05057 [astro-ph.CO]

Lee JW, Lim S (2010) Minimum mass of galaxies from BEC or scalar field dark matter. JCAP 01:007. https://doi.org/10.1088/1475-7516/2010/01/007. arXiv:0812.1342 [astro-ph]

Lee T, Pang Y (1992) Nontopological solitons. Phys Rep 221:251-350. https://doi.org/10.1016/03701573(92)90064-7

Lelli F, McGaugh SS, Schombert JM (2016) SPARC: mass models for 175 disk galaxies with spitzer photometry and accurate rotation curves. Astron J 152:157. https://doi.org/10.3847/0004-6256/152/ 6/157. arXiv:1606.09251 [astro-ph.GA]

Lelli F, McGaugh SS, Schombert JM, Pawlowski MS (2017) One law to rule them all: the radial acceleration relation of galaxies. Astrophys J 836(2):152. https://doi.org/10.3847/1538-4357/836/2/152. arXiv:1610.08981 [astro-ph.GA]

Leo M, Theuns T, Baugh CM, Li B, Pascoli S (2020) Constraining structure formation using EDGES. JCAP 04:004. https://doi.org/10.1088/1475-7516/2020/04/004. arXiv:1909.04641 [astro-ph.CO]

Leong KH, Schive HY, Zhang UH, Chiueh T (2019) Testing extreme-axion wave-like dark matter using the BOSS Lyman-alpha forest data. MNRAS 484(3):4273-4286. https://doi.org/10.1093/mnras/stz271. arXiv:1810.05930 [astro-ph.CO]

Lesgourgues J (2011) The cosmic linear anisotropy solving system (CLASS) I: overview. arXiv:1104.2932 [astro-ph.IM] (arXiv e-prints)

Lesgourgues J, Tram T (2011) The cosmic linear anisotropy solving system (CLASS) IV: efficient implementation of non-cold relics. JCAP 09:032. https://doi.org/10.1088/1475-7516/2011/09/032. arXiv:1104.2935 [astro-ph.CO]

Leung E, Broadhurst T, Lim J, Diego JM, Chiueh T, Schive HY, Windhorst R (2018) Magnification bias of distant galaxies in the Hubble Frontier Fields: testing wave versus particle dark matter predictions. Astrophys J 862(2):156. https://doi.org/10.3847/1538-4357/aacdad. arXiv:1806.07905 [astro-ph.GA]

Levkov D, Panin A, Tkachev I (2018) Gravitational Bose-Einstein condensation in the kinetic regime. Phys Rev Lett 121(15):151301. https://doi.org/10.1103/PhysRevLett.121.151301. arXiv:1804.05857 [astro-ph.CO]

Lewis A, Challinor A, Lasenby A (2000) Efficient computation of CMB anisotropies in closed FRW models. Astrophys J 538:473-476. https://doi.org/10.1086/309179. arXiv:astro-ph/9911177

Li X, Hui L, Bryan GL (2019) Numerical and perturbative computations of the fuzzy dark matter model. Phys Rev D 99(6):063509. https://doi.org/10.1103/PhysRevD.99.063509. arXiv:1810.01915 [astroph.CO]

Lidz A, Hui L (2018) Implications of a prereionization 21-cm absorption signal for fuzzy dark matter. Phys Rev D 98(2):023011. https://doi.org/10.1103/PhysRevD.98.023011. arXiv:1805.01253 [astro-ph.CO]

Lin T (2019) Dark matter models and direct detection. PoS 333:009. https://doi.org/10.22323/1.333.0009. arXiv:1904.07915 [hep-ph] 
Lisanti M, Moschella M, Outmezguine NJ, Slone O (2019a) Testing dark matter and modifications to gravity using local milky way observables. Phys Rev D 100(8):083009. https://doi.org/10.1103/PhysRevD. 100.083009. arXiv:1812.08169 [astro-ph.GA]

Lisanti M, Moschella M, Outmezguine NJ, Slone O (2019b) The inconsistency of superfluid dark matter with Milky Way dynamics. arXiv:1911.12365 [hep-ph] (arXiv e-prints)

London F (1938) The $\lambda$-phenomenon of liquid helium and the Bose-Einstein degeneracy. Nature 141(3571):643-644. https://doi.org/10.1038/141643a0

López ULA, Gonzalez-Morales AX (2016) Towards accurate cosmological predictions for rapidly oscillating scalar fields as dark matter. JCAP 07:048. https://doi.org/10.1088/1475-7516/2016/07/048. arXiv:1511.08195 [astro-ph.CO]

Lopez-Honorez L, Mena O, Villanueva-Domingo P (2019) Dark matter microphysics and $21 \mathrm{~cm}$ observations. Phys Rev D 99(2):023522. https://doi.org/10.1103/PhysRevD.99.023522. arXiv:1811.02716 [astro-ph.CO]

Lotz JM et al (2017) The frontier fields: survey design. Astrophys J 837:97

Lozanov KD, Amin MA (2019) Gravitational perturbations from oscillons and transients after inflation. Phys Rev D 99(12):123504. https://doi.org/10.1103/PhysRevD.99.123504. arXiv:1902.06736 [astroph.CO]

Lvov Y, Nazarenko S, West R (2003) Wave turbulence in Bose-Einstein condensates. Phys D 184(1-4):333351. https://doi.org/10.1016/S0167-2789(03)00239-2. arXiv:math-ph/0308008 [math-ph]

Madau P, Shen S, Governato F (2014) Dark matter heating and early core formation in dwarf galaxies. Astrophys J Lett 789:L17. https://doi.org/10.1088/2041-8205/789/1/L17. arXiv:1405.2577 [astroph.GA]

Madelung E (1927) Quantentheorie in hydrodynamischer form. Z Phys 40(3-4):322-326. https://doi.org/ 10.1007/BF01400372

Madison KW, Chevy F, Wohlleben W, Dalibard J (2000) Vortex formation in a stirred BoseEinstein condensate. Phys Rev Lett 84:806-809. https://doi.org/10.1103/PhysRevLett.84.806. arXiv:cond-mat/9912015

Mahan GD (2000) Many-particle physics, physics of solids and liquids, 3rd edn. Springer, Boston. https:// doi.org/10.1007/978-1-4757-5714-9

Mahdavi A, Hoekstra H, Babul A, Balam D, Capak P (2007) A dark core in Abell 520. Astrophys J 668:806-814. https://doi.org/10.1086/521383. arXiv:0706.3048 [astro-ph]

Maleki A, Baghram S, Rahvar S (2020) Investigation of two colliding solitonic cores in Fuzzy Dark Matter models. Phys Rev D 101(2):023508. https://doi.org/10.1103/PhysRevD.101.023508. arXiv:1911.00486 [astro-ph.CO]

Mao S, Schneider P (1998) Evidence for substructure in lens galaxies? MNRAS 295:587-594. https://doi. org/10.1046/j.1365-8711.1998.01319.x. arXiv:astro-ph/9707187

Marchesini D, D’Onghia E, Chincarini G, Firmani C, Conconi P, Molinari E, Zacchei A (2002) Halpha rotation curves: the soft core question. Astrophys J 575:801-813. https://doi.org/10.1086/341475. arXiv:astro-ph/0202075

Marsh DJ (2018) Axions and ALPs: a very short introduction. In: 13th Patras workshop on axions, WIMPs and WISPs, pp 59-74. https://doi.org/10.3204/DESY-PROC-2017-02/marsh_david. arXiv:1712.03018 [hep-ph]

Marsh DJE (2016a) Axion cosmology. Phys Rep 643:1-79. https://doi.org/10.1016/j.physrep.2016.06.005. arXiv:1510.07633 [astro-ph.CO]

Marsh DJE (2016b) WarmAndFuzzy: the halo model beyond CDM. arXiv:1605.05973 [astro-ph.CO] (arXiv e-prints)

Marsh DJE, Niemeyer JC (2019) Strong constraints on fuzzy dark matter from ultrafaint dwarf galaxy Eridanus II. Phys Rev Lett 123(5):051103. https://doi.org/10.1103/PhysRevLett.123.051103. arXiv:1810.08543 [astro-ph.CO]

Marsh DJE, Silk J (2014) A model for halo formation with axion mixed dark matter. MNRAS 437(3):2652_ 2663. https://doi.org/10.1093/mnras/stt2079. arXiv:1307.1705 [astro-ph.CO]

Massey R, Kitching T, Nagai D (2011) Cluster bulleticity. MNRAS 413:1709. https://doi.org/10.1111/j. 1365-2966.2011.18246.x. arXiv:1007.1924 [astro-ph.CO]

Matos T, Guzmán FS (2000) Scalar fields as dark matter in spiral galaxies. Class Quantum Grav 17:L9-L16. https://doi.org/10.1088/0264-9381/17/1/102. arXiv:gr-qc/9810028

Matos T, Vazquez-Gonzalez A, Magana J (2009) $\phi^{2}$ as dark matter. MNRAS 393:1359-1369. https://doi. org/10.1111/j.1365-2966.2008.13957.x. arXiv:0806.0683 [astro-ph] 
McConnachie AW (2012) The observed properties of dwarf galaxies in and around the Local Group. Astron J 144:4. https://doi.org/10.1088/0004-6256/144/1/4. arXiv:1204.1562 [astro-ph.CO]

McGaugh S (2020) Predictions and outcomes for the dynamics of rotating galaxies. Galaxies 8:35. https:// doi.org/10.3390/galaxies8020035. arXiv:2004.14402 [astro-ph.GA]

McGaugh S, Lelli F, Schombert J (2016) Radial acceleration relation in rotationally supported galaxies. Phys Rev Lett 117(20):201101. https://doi.org/10.1103/PhysRevLett.117.201101. arXiv:1609.05917 [astro-ph.GA]

McGaugh SS (2005) The baryonic Tully-Fisher relation of galaxies with extended rotation curves and the stellar mass of rotating galaxies. Astrophys J 632:859-871. https://doi.org/10.1086/432968. arXiv:astro-ph/0506750

McGaugh SS (2008) The halo by halo missing baryon problem. IAU Symp 244:136-145. https://doi.org/ 10.1017/S1743921307013920. arXiv:0707.3795 [astro-ph]

McGaugh SS, de Blok WJG (1998) Testing the dark matter hypothesis with low surface brightness galaxies and other evidence. Astrophys J 499:41. https://doi.org/10.1086/305612. arXiv:astro-ph/9801123

Menci N, Merle A, Totzauer M, Schneider A, Grazian A, Castellano M, Sanchez NG (2017) Fundamental physics with the Hubble Frontier Fields: constraining dark matter models with the abundance of extremely faint and distant galaxies. Astrophys J 836(1):61. https://doi.org/10.3847/1538-4357/836/ 1/61. arXiv:1701.01339 [astro-ph.CO]

Mendonça JT, Bingham R, Shukla PK (2005) A kinetic approach to Bose-Einstein condensates: self-phase modulation and Bogoliubov oscillations. Sov Phys JETP 101(5):942-948. https://doi.org/10.1134/1. 2149073. arXiv:nlin/0502059 [nlin.PS]

Milgrom M (1983a) A modification of the Newtonian dynamics as a possible alternative to the hidden mass hypothesis. Astrophys J 270:365-370. https://doi.org/10.1086/161130

Milgrom M (1983b) A modification of the Newtonian dynamics: implications for galaxies. Astrophys J 270:371-383. https://doi.org/10.1086/161131

Milgrom M (1983c) A modification of the Newtonian dynamics: implications for galaxy systems. Astrophys J 270:384-389. https://doi.org/10.1086/161132

Misner C (1972) Stability of Kerr black holes against scalar perturbations. Bull Am Phys Soc 17:472

Mocz P, Succi S (2015) Numerical solution of the nonlinear Schrödinger equation using smoothed-particle hydrodynamics. Phys Rev E 91:5. https://doi.org/10.1103/PhysRevE.91.053304

Mocz P, Vogelsberger M, Robles VH, Zavala J, Boylan-Kolchin M, Fialkov A, Hernquist L (2017) Galaxy formation with BECDM-I. Turbulence and relaxation of idealized haloes. MNRAS 471(4):4559-4570. https://doi.org/10.1093/mnras/stx1887. arXiv:1705.05845 [astro-ph.CO]

Mocz P, Lancaster L, Fialkov A, Becerra F, Chavanis PH (2018) Schrödinger-Poisson-VlasovPoisson correspondence. Phys Rev D 97(8):083519. https://doi.org/10.1103/PhysRevD.97.083519. arXiv:1801.03507 [astro-ph.CO]

Mocz P et al (2019) First star-forming structures in fuzzy cosmic filaments. Phys Rev Lett 123(14):141301. https://doi.org/10.1103/PhysRevLett.123.141301. arXiv:1910.01653 [astro-ph.GA]

Moore B (1994) Evidence against dissipationless dark matter from observations of galaxy haloes. Nature 370:629. https://doi.org/10.1038/370629a0

Mukhanov V (2005) Physical foundations of cosmology. Cambridge University Press, Cambridge. https:// doi.org/10.2277/0521563984

Muñoz JB, Dvorkin C, Cyr-Racine FY (2020) Probing the small-scale matter power spectrum with large-scale 21-cm data. Phys Rev D 101(6):063526. https://doi.org/10.1103/PhysRevD.101.063526. arXiv:1911.11144 [astro-ph.CO]

Munshi F, Governato F, Brooks AM, Christensen C, Shen S, Loebman S, Moster B, Quinn T, Wadsley J (2013) Reproducing the Stellar mass/Halo mass relation in simulated $\Lambda$ CDM galaxies: theory versus observational estimates. Astrophys J 766:56. https://doi.org/10.1088/0004-637X/766/1/56. arXiv:1209.1389 [astro-ph.CO]

Nadler EO, Gluscevic V, Boddy KK, Wechsler RH (2019) Constraints on dark matter microphysics from the milky way satellite population. Astrophys J Lett 878(2):32. https://doi.org/10.3847/2041-8213/ ab1eb2 [Erratum: Astrophys J Lett 897, L46 (2020)]. arXiv:1904.10000 [astro-ph.CO]

Nambu Y, Jona-Lasinio G (1961) Dynamical model of elementary particles based on an analogy with superconductivity. I. Phys Rev 122:345-358. https://doi.org/10.1103/PhysRev.122.345

Kuzio de Naray R, McGaugh SS, de Blok WJG, Bosma A (2006) High resolution optical velocity fields of 11 low surface brightness galaxies. Astrophys J Suppl 165:461-479. https://doi.org/10.1086/505345. arXiv:astro-ph/0604576 
Kuzio de Naray R, McGaugh SS, de Blok WJG (2008) Mass models for low surface brightness galaxies with high resolution optical velocity fields. Astrophys J 676:920-943. https://doi.org/10.1086/527543. arXiv:0712.0860 [astro-ph]

Navarro JF (2019) The Origin of Galaxy Scaling Laws in LCDM. In: Essig R, Feng J, Zurek K (eds) Illuminating Dark Matter. Astrophysics and Space Science Proceedings, vol 56. Springer, Cham. https://doi.org/10.1007/978-3-030-31593-1_13. arXiv:1811.02025 [astro-ph.GA]

Navarro JF, Frenk CS, White SDM (1997) A universal density profile from hierarchical clustering. Astrophys J 490:493-508. https://doi.org/10.1086/304888. arXiv:astro-ph/9611107

Navarro JF, Hayashi E, Power C, Jenkins A, Frenk CS, White SDM, Springel V, Stadel J, Quinn TR (2004) The inner structure of $\Lambda \mathrm{CDM}$ haloes - III. Universality and asymptotic slopes. MNRAS 349:1039. https://doi.org/10.1111/j.1365-2966.2004.07586.x. arXiv:astro-ph/0311231

Navarro JF, Benítez-Llambay A, Fattahi A, Frenk CS, Ludlow AD, Oman KA, Schaller M, Theuns T (2017) The origin of the mass discrepancy-acceleration relation in 1 CDM. MNRAS 471(2):18411848. https://doi.org/10.1093/mnras/stx1705. arXiv:1612.06329 [astro-ph.GA]

Navez P (2005) Kinetic theory and dynamic structure factor of a condensate in the random phase approximation. J Low Temp Phys 138(3-4):705-710. https://doi.org/10.1007/s10909-005-2290-3. arXiv:cond-mat/0406033 [cond-mat.stat-mech]

Nazarenko S (2011a) Introduction. In: Wave Turbulence. Lecture Notes in Physics, vol 825. Springer, Berlin, pp 1-14. https://doi.org/10.1007/978-3-642-15942-8_1

Nazarenko S, Onorato M (2006) Wave turbulence and vortices in Bose-Einstein condensation. Phys D 219(1):1-12. https://doi.org/10.1016/j.physd.2006.05.007. arXiv:nlin/0507051 [nlin.CD]

Nazarenko S (2011b) Wave Turbulence Formalism. In: Wave Turbulence. Lecture Notes in Physics, vol 825. Springer, Berlin, pp 67-105. https://doi.org/10.1007/978-3-642-15942-8_6

Nebrin O, Ghara R, Mellema G (2019) Fuzzy dark matter at cosmic dawn: new 21-cm constraints. JCAP 04:051. https://doi.org/10.1088/1475-7516/2019/04/051. arXiv:1812.09760 [astro-ph.CO]

Ni Y, Wang MY, Feng Y, Di Matteo T (2019) Predictions for the abundance of high-redshift galaxies in a fuzzy dark matter universe. Mon Not R Astron Soc 488(4):5551-5565. https://doi.org/10.1093/mnras/ stz2085. arXiv:1904.01604 [astro-ph.CO]

Nicol EJ, Carbotte JP (2005) Properties of the superconducting state in a two-band model. Phys Rev B 71(5):054501. https://doi.org/10.1103/PhysRevB.71.054501. arXiv:cond-mat/0409335 [condmat.supr-con]

Nicolis A (2011) Low-energy effective field theory for finite-temperature relativistic superfluids. arXiv:1108.2513 [hep-th] (arXiv e-prints)

Niemeyer JC (2019) Small-scale structure of fuzzy and axion-like dark matter. Prog Part Nucl Phys. https:// doi.org/10.1016/j.ppnp.2020.103787. arXiv:1912.07064 [astro-ph.CO]

Noordermeer E, van der Hulst JM, Sancisi R, Swaters RS, van Albada TS (2007) The mass distribution in early-type disk galaxies: declining rotation curves and correlations with optical properties. MNRAS 376:1513-1546. https://doi.org/10.1111/j.1365-2966.2007.11533.x. arXiv:astro-ph/0701731

Nori M, Baldi M (2018) AX-GADGET: a new code for cosmological simulations of fuzzy dark matter and axion models. MNRAS 478(3):3935-3951. https://doi.org/10.1093/mnras/sty1224. arXiv:1801.08144 [astro-ph.CO]

Nori M, Murgia R, Iršič V, Baldi M, Viel M (2019) Lyman $\alpha$ forest and non-linear structure characterization in fuzzy dark matter cosmologies. MNRAS 482(3):3227-3243. https://doi.org/10.1093/mnras/ sty2888. arXiv:1809.09619 [astro-ph.CO]

Oh SH, de Blok WJG, Brinks E, Walter F, Kennicutt J, Robert C (2011) Dark and luminous matter in THINGS dwarf galaxies. Astron J 141:193. https://doi.org/10.1088/0004-6256/141/6/193. arXiv:1011.0899 [astro-ph.CO]

Oh SH et al (2015) High-resolution mass models of dwarf galaxies from LITTLE THINGS. Astron J 149:180. https://doi.org/10.1088/0004-6256/149/6/180. arXiv:1502.01281 [astro-ph.GA]

Oman KA et al (2015) The unexpected diversity of dwarf galaxy rotation curves. MNRAS 452(4):36503665. https://doi.org/10.1093/mnras/stv1504. arXiv:1504.01437 [astro-ph.GA]

Oñorbe J, Boylan-Kolchin M, Bullock JS, Hopkins PF, Kerěs D, Faucher-Giguère CA, Quataert E, Murray N (2015) Forged in FIRE: cusps, cores, and baryons in low-mass dwarf galaxies. MNRAS 454(2):20922106. https://doi.org/10.1093/mnras/stv2072. arXiv:1502.02036 [astro-ph.GA]

Ostriker EC (1999) Dynamical friction in a gaseous medium. Astrophys J 513:252. https://doi.org/10.1086/ 306858. arXiv:astro-ph/9810324 
Ostriker JP, Choi E, Chow A, Guha K (2019) Mind the gap: is the too big to fail problem resolved? Astrophys J 885(1):97. https://doi.org/10.3847/1538-4357/ab3288. arXiv:1904.10471 [astro-ph.GA]

Papastergis E, Giovanelli R, Haynes MP, Shankar F (2015) Is there a "too big to fail" problem in the field? Astron Astrophys 574:A113. https://doi.org/10.1051/0004-6361/201424909. arXiv:1407.4665 [astro-ph.GA]

Peccei RD, Quinn HR (1977) CP conservation in the presence of pseudoparticles. Phys Rev Lett 38:14401443. https://doi.org/10.1103/PhysRevLett.38.1440

Peebles PJE (1969) Origin of the angular momentum of galaxies. Astrophys J 155:393. https://doi.org/10. $1086 / 149876$

Peebles PJE (1993) Principles of physical cosmology. Princeton University Press, Princeton

Peebles PJE (2000) Fluid dark matter. Astrophys J Lett 534:L127. https://doi.org/10.1086/312677. arXiv:astro-ph/0002495

Penarrubia J, Navarro JF, McConnachie AW (2008) The tidal evolution of local group dwarf spheroidals. Astrophys J 673:226. https://doi.org/10.1086/523686. arXiv:0708.3087 [astro-ph]

Penrose O, Onsager L (1956) Bose-Einstein condensation and liquid helium. Phys Rev 104:576-584. https:// doi.org/10.1103/PhysRev.104.576

Pitaevskii L, Stringari S (2016) Bose-Einstein condensation and superfluidity. Oxford University Press, Oxford, International series of monographs on physics. https://doi.org/10.1093/acprof:oso/ 9780198758884.001 .0001

Powell AJ (2016) The cosmology and astrophysics of axion-like particles. PhD thesis, Oxford University

Preskill J, Wise MB, Wilczek F (1983) Cosmology of the invisible axion. Phys Lett B 120:127-132. https:// doi.org/10.1016/0370-2693(83)90637-8

Randall SW, Markevitch M, Clowe D, Gonzalez AH, Bradac M (2008) Constraints on the self-interaction cross-section of dark matter from numerical simulations of the merging galaxy cluster 1E 0657-56. Astrophys J 679:1173-1180. https://doi.org/10.1086/587859. arXiv:0704.0261 [astro-ph]

Read JI, Walker MG, Steger P (2019) Dark matter heats up in dwarf galaxies. MNRAS 484(1):1401-1420. https://doi.org/10.1093/mnras/sty3404. arXiv:1808.06634 [astro-ph.GA]

Rindler-Daller T, Shapiro PR (2012) Angular momentum and vortex formation in Bose-Einstein-condensed cold dark matter haloes. MNRAS 422:135-161. https://doi.org/10.1111/j.1365-2966.2012.20588.x. arXiv:1106.1256 [astro-ph.CO]

Ringwald A (2014) Axions and axion-like particles. In: 49th rencontres de moriond on electroweak interactions and unified theories, pp 223-230. arXiv:1407.0546 [hep-ph]

Rogel-Salazar J, Choi S, New GHC, Burnett K (2004) Methods of quantum field theory for trapped BoseEinstein condensates. J Opt B 6(9):R33-R59. https://doi.org/10.1088/1464-4266/6/9/R01

Rogers KK, Peiris HV (2020) Strong bound on canonical ultra-light axion dark matter from the Lyman-alpha forest. Phys. Rev. Lett. 126:071302. https://doi.org/10.1103/PhysRevLett.126.071302 arXiv:2007.12705 [astro-ph.CO]

Romano-Diaz E, Garaldi E, Borzyszkowski M, Porciani C (2017) ZOMG-II. Does the halo assembly history influence central galaxies and gas accretion? MNRAS 469(2):1809-1823. https://doi.org/10. 1093/mnras/stx878. arXiv:1701.02743 [astro-ph.GA]

Roszkowski L, Sessolo EM, Trojanowski S (2018) WIMP dark matter candidates and searches-current status and future prospects. Rep Prog Phys 81(6):066201. https://doi.org/10.1088/1361-6633/aab913. arXiv: 1707.06277 [hep-ph]

Rubin VC, Ford JWK (1970) Rotation of the Andromeda Nebula from a spectroscopic survey of emission regions. Astrophys J 159:379-403. https://doi.org/10.1086/150317

Ruffini R, Bonazzola S (1969a) Systems of self-gravitating particles in general relativity and the concept of an equation of state. Phys Rev 187:1767-1783. https://doi.org/10.1103/PhysRev.187.1767

Safarzadeh M, Spergel DN (2019) Ultra-light dark matter is incompatible with the Milky Way's dwarf satellites. Astrophys J. https://doi.org/10.3847/1538-4357/ab7db2. arXiv:1906.11848 [astro-ph.CO]

Safarzadeh M, Scannapieco E, Babul A (2018) A limit on the warm dark matter particle mass from the redshifted $21 \mathrm{~cm}$ absorption line. Astrophys J Lett 859(2):L18. https://doi.org/10.3847/2041-8213/ aac5e0. arXiv:1803.08039 [astro-ph.CO]

Sanders RH, McGaugh SS (2002) Modified Newtonian Dynamics as an alternative to dark matter. Annu Rev Astron Astrophys 40:263-317. https://doi.org/10.1146/annurev.astro.40.060401.093923. arXiv:astro-ph/0204521

Sawala T et al (2016) The APOSTLE simulations: solutions to the Local Group's cosmic puzzles. MNRAS 457(2):1931-1943. https://doi.org/10.1093/mnras/stw145. arXiv:1511.01098 [astro-ph.GA] 
Schay G (1924) Zur Quantentheorie der einatomigen idealen Gase. Z Phys 25(1):37-41. https://doi.org/10. 1007/BF01327507

Schaye $\mathrm{J}$ et al (2015) The EAGLE project: simulating the evolution and assembly of galaxies and their environments. MNRAS 446:521-554. https://doi.org/10.1093/mnras/stu2058. arXiv:1407.7040 [astro-ph.GA]

Schive HY, Chiueh T, Broadhurst T (2014a) Cosmic structure as the quantum interference of a coherent dark wave. Nat Phys 10:496-499. https://doi.org/10.1038/nphys2996. arXiv:1406.6586 [astro-ph.GA]

Schive HY, Liao MH, Woo TP, Wong SK, Chiueh T, Broadhurst T, Hwang WYP (2014b) Understanding the Core-Halo relation of quantum wave dark matter from 3D simulations. Phys Rev Lett 113(26):261302. https://doi.org/10.1103/PhysRevLett.113.261302. arXiv:1407.7762 [astro-ph.GA]

Schive HY, Chiueh T, Broadhurst T, Huang KW (2016) Contrasting galaxy formation from quantum wave dark matter, $\psi \mathrm{DM}$, with $\Lambda \mathrm{CDM}$, using Planck and Hubble data. Astrophys J 818(1):89. https://doi. org/10.3847/0004-637X/818/1/89. arXiv:1508.04621 [astro-ph.GA]

Schive HY, Chiueh T, Broadhurst T (2020) Soliton random walk and the cluster-stripping problem in ultralight dark matter. Phys Rev Lett 124:201301. https://doi.org/10.1103/PhysRevLett.124.201301. arXiv:1912.09483 [astro-ph.GA]

Schmitt A (2015) Introduction to superfluidity: field-theoretical approach and applications. Lecture Notes in Physics, vol 888. Springer, Cham. https://doi.org/10.1007/978-3-319-07947-9. arXiv:1404.1284 [hep-ph]

Schneider A (2015) Structure formation with suppressed small-scale perturbations. MNRAS 451(3):31173130. https://doi.org/10.1093/mnras/stv1169. arXiv:1412.2133 [astro-ph.CO]

Schneider A (2018) Constraining noncold dark matter models with the global 21-cm signal. Phys Rev D 98(6):063021. https://doi.org/10.1103/PhysRevD.98.063021. arXiv:1805.00021 [astro-ph.CO]

Schneider MD, Frenk CS, Cole S (2012) The shapes and alignments of dark matter halos. JCAP 5:030. https://doi.org/10.1088/1475-7516/2012/05/030. arXiv:1111.5616 [astro-ph.CO]

Schneider P (2015) Extragalactic astronomy and cosmology: an introduction, 2nd edn. Springer, Heidelberg. https://doi.org/10.1007/978-3-642-54083-7

Schutz K (2020) The Subhalo mass function and ultralight bosonic dark matter. arXiv:2001.05503 [astroph.CO] (arXiv e-prints)

Schwabe B, Niemeyer JC, Engels JF (2016) Simulations of solitonic core mergers in ultralight axion dark matter cosmologies. Phys Rev D 94(4):043513. https://doi.org/10.1103/PhysRevD.94.043513. arXiv:1606.05151 [astro-ph.CO]

Schwabe B, Gosenca M, Behrens C, Niemeyer JC, Easther R (2020) Simulating mixed fuzzy and cold dark matter. Phys Rev D 102:083518. https://doi.org/10.1103/PhysRevD.102.083518. arXiv:2007.08256 [astro-ph.CO]

Seidel E, Suen WM (1990) Dynamical evolution of boson stars. 1. Perturbing the ground state. Phys Rev D 42:384-403. https://doi.org/10.1103/PhysRevD.42.384

Semikoz D, Tkachev I (1997) Condensation of bosons in kinetic regime. Phys Rev D 55:489-502. https:// doi.org/10.1103/PhysRevD.55.489. arXiv:hep-ph/9507306

Sharma A, Khoury J, Lubensky T (2019) The equation of state of dark matter superfluids. JCAP 05:054. https://doi.org/10.1088/1475-7516/2019/05/054. arXiv:1809.08286 [hep-th]

Sheth RK, Tormen G (1999) Large-scale bias and the peak background split. MNRAS 308(1):119-126. https://doi.org/10.1046/j.1365-8711.1999.02692.x. arXiv:astro-ph/9901122 [astro-ph]

Shimabukuro H, Ichiki K, Kadota K (2020) Constraining the nature of ultra light dark matter particles with the $21 \mathrm{~cm}$ forest. Phys Rev D 101(4):043516. https://doi.org/10.1103/PhysRevD.101.043516. arXiv:1910.06011 [astro-ph.CO]

Sikivie P (2008) Axion cosmology. In: Kuster M, Raffelt G, Beltran B (eds) Axions: theory, cosmology, and experimental searches. Lecture Notes in Physics, vol 741. Springer, Berlin, pp 19-50. https://doi. org/10.1007/978-3-540-73518-2_2. arXiv:astro-ph/0610440

Sikivie P (2020) Invisible axion search methods. Rev Mod Phys 93:015004. https://doi.org/10.1103/ RevModPhys.93.015004 arXiv:2003.02206 [hep-ph]

Sikivie P, Yang Q (2009) Bose-Einstein condensation of dark matter axions. Phys Rev Lett 103. https://doi. org/10.1103/PhysRevLett.103.111301. arXiv:0901.1106 [hep-ph]

Silverman MP, Mallett RL (2002) Dark matter as a cosmic Bose-Einstein condensate and possible superfluid. Gen Relat Grav 34:633-649. https://doi.org/10.1023/A:1015934027224 
Simon JD, Bolatto AD, Leroy A, Blitz L, Gates EL (2005) High-resolution measurements of the halos of four dark matter-dominated galaxies: deviations from a universal density profile. Astrophys J 621:757-776. https://doi.org/10.1086/427684. arXiv:astro-ph/0412035

Sin SJ (1994) Late time cosmological phase transition and galactic halo as Bose liquid. Phys Rev D 50:3650-3654. https://doi.org/10.1103/PhysRevD.50.3650. arXiv:hep-ph/9205208

Skordis C (2009) The tensor-vector-scalar theory and its cosmology. Class Quantum Grav 26(14):143001. https://doi.org/10.1088/0264-9381/26/14/143001. arXiv:0903.3602 [astro-ph.CO]

Skordis C, Mota D, Ferreira P, Boehm C (2006) Large scale structure in Bekenstein's theory of relativistic Modified Newtonian Dynamics. Phys Rev Lett 96:011301. https://doi.org/10.1103/PhysRevLett.96. 011301. arXiv:astro-ph/0505519

Slepian Z, Goodman J (2012) Ruling out bosonic repulsive dark matter in thermal equilibrium. MNRAS 427:839. https://doi.org/10.1111/j.1365-2966.2012.21901.x. arXiv:1109.3844 [astro-ph.CO]

Son DT (2002) Low-energy quantum effective action for relativistic superfluids. arXiv:hep-ph/0204199 (arXiv e-prints)

Son DT, Wingate M (2006) General coordinate invariance and conformal invariance in nonrelativistic physics: unitary fermi gas. Ann Phys 321:197-224. https://doi.org/10.1016/j.aop.2005.11.001. arXiv:cond-mat/0509786

Song M (2016) The evolution of the galaxy Stellar mass function at $z=4-8$ : a steepening low-massend slope with increasing redshift. Astrophys J 825(1):5. https://doi.org/10.3847/0004-637X/825/1/ 5. arXiv:1507.05636 [astro-ph.GA]

Spergel DN, Steinhardt PJ (2000) Observational evidence for selfinteracting cold dark matter. Phys Rev Lett 84:3760-3763. https://doi.org/10.1103/PhysRevLett.84.3760. arXiv:astro-ph/9909386

Spergel DN, Flauger R, Hložek R (2015) Planck data reconsidered. Phys Rev D 91(2):023518. https://doi. org/10.1103/PhysRevD.91.023518. arXiv:1312.3313 [astro-ph.CO]

Springel V, Farrar G (2007) The speed of the bullet in the merging galaxy cluster 1E0657-56. MNRAS 380:911-925. https://doi.org/10.1111/j.1365-2966.2007.12159.x. arXiv:astro-ph/0703232

Springel V, Wang J, Vogelsberger M, Ludlow A, Jenkins A, Helmi A, Navarro JF, Frenk CS, White SD (2008) The Aquarius project: the subhalos of galactic halos. MNRAS 391:1685-1711. https://doi.org/ 10.1111/j.1365-2966.2008.14066.x. arXiv:0809.0898 [astro-ph]

Starobinsky AA (1973) Amplification of waves reflected from a rotating "black hole". Sov Phys JETP 37(1):28-32

Stott MJ, Marsh DJE (2018) Black hole spin constraints on the mass spectrum and number of axionlike fields. Phys Rev D 98(8):083006. https://doi.org/10.1103/PhysRevD.98.083006. arXiv:1805.02016 [hep-ph]

Suárez A, Robles VH, Matos T (2014) A review on the scalar field/Bose-Einstein condensate dark matter model. In: Moreno González C, Madriz Aguilar J, Reyes Barrera L (eds) Accelerated Cosmic Expansion. Astrophysics and Space Science Proceedings, vol 38. Springer, Cham. pp 107-142. https://doi. org/10.1007/978-3-319-02063-1_9. arXiv:1302.0903 [astro-ph.CO]

Sun C, Jia S, Barsi C, Rica S, Picozzi A, Fleischer JW (2012) Observation of the kinetic condensation of classical waves. Nat Phys 8(6):471-475. https://doi.org/10.1038/nphys2278

Sunyaev RA, Zeldovich YB (1980) The velocity of clusters of galaxies relative to the microwave background-the possibility of its measurement. MNRAS 190:413-420. https://doi.org/10.1093/ mnras/190.3.413

Tamura N et al (2016) Prime focus spectrograph (PFS) for the Subaru telescope: overview, recent progress, and future perspectives. Proc SPIE 9908:99081M. https://doi.org/10.1117/12.2232103. arXiv:1608.01075 [astro-ph.IM]

Taylor E, Griffin A (2005) Two-fluid hydrodynamic modes in a trapped superfluid gas. Phys Rev A 72(5):053630. https://doi.org/10.1103/PhysRevA.72.053630. arXiv:cond-mat/0509040 [condmat.stat-mech]

Tegmark M et al (2004) The 3-D power spectrum of galaxies from the SDSS. Astrophys J 606:702-740. https://doi.org/10.1086/382125. arXiv:astro-ph/0310725

Tollet E et al. (2016) NIHAO-IV: core creation and destruction in dark matter density profiles across cosmic time. MNRAS 456(4), 3542-3552. https://doi.org/10.1093/mnras/stv2856 [Erratum: MNRAS 487, 1764 (2019)]. arXiv:1507.03590 [astro-ph.GA]

Tommasini P, de Passos EJ, de Toledo Piza AF, Hussein MS, Timmermans E (2003) Bogoliubov theory for mutually coherent condensates. Phys Rev A 67(2):023606. https://doi.org/10.1103/PhysRevA.67. 023606 
Tsubota M, Kasamatsu K, Kobayashi M (2013a) Quantized vortices in superfluid helium and atomic BoseEinstein condensates. In: Bennemann, KH and Ketterson, JB (eds) Novel Superfluids, vol 1, Oxford University Press. p 156-252. arXiv:1004.5458 [cond-mat.quant-gas]

Tsubota M, Kobayashi M, Takeuchi H (2013b) Quantum hydrodynamics. Phys Rep 522(3):191-238. https:// doi.org/10.1016/j.physrep.2012.09.007. arXiv:1208.0422 [cond-mat.other]

Tully RB, Shaya EJ, Karachentsev ID, Courtois HM, Kocevski DD, Rizzi L, Peel A (2008) Our peculiar motion away from the local void. Astrophys J 676:184-205. https://doi.org/10.1086/527428. arXiv:0705.4139 [astro-ph]

Ureña-López LA (2019) Brief review on scalar field dark matter models. Front Astron Space Sci 6:47. https://doi.org/10.3389/fspas.2019.00047

Usui A, Takeuchi H (2015) Rabi-coupled countersuperflow in binary Bose-Einstein condensates. Phys Rev A 91(6):063635. https://doi.org/10.1103/PhysRevA.91.063635. arXiv:1503.04010 [cond-mat.quantgas]

Vale A, Ostriker J (2004) Linking halo mass to galaxy luminosity. MNRAS 353:189. https://doi.org/10. 1111/j.1365-2966.2004.08059.x. arXiv:astro-ph/0402500

van den Bosch FC, Swaters RA (2001) Dwarf galaxy rotation curves and the core problem of dark matter halos. MNRAS 325:1017. https://doi.org/10.1046/j.1365-8711.2001.04456.x. arXiv:astro-ph/0006048

van Haarlem MP et al (2013) LOFAR: the low-frequency array. Astron Astrophys 556:A2. https://doi.org/ 10.1051/0004-6361/201220873. arXiv:1305.3550 [astro-ph.IM]

Vegetti S, Koopmans LVE (2009a) Bayesian strong gravitational-lens modelling on adaptive grids: objective detection of mass substructure in galaxies. MNRAS 392:945. https://doi.org/10.1111/j.1365-2966. 2008.14005.x. arXiv:0805.0201 [astro-ph]

Vegetti S, Koopmans LVE (2009b) Statistics of CDM substructure from strong gravitational lensing: quantifying the mass fraction and mass function. MNRAS 400:1583-1592. https://doi.org/10.1111/j.13652966.2009.15559.x. arXiv:0903.4752 [astro-ph.CO]

Vegetti S, Koopmans LVE, Bolton A, Treu T, Gavazzi R (2010) Detection of a dark substructure through gravitational imaging. MNRAS 408:1969. https://doi.org/10.1111/j.1365-2966.2010.16865. X. arXiv:0910.0760 [astro-ph.CO]

Veltmaat J, Niemeyer JC (2016) Cosmological particle-in-cell simulations with ultralight axion dark matter. Phys Rev D 94(12):123523. https://doi.org/10.1103/PhysRevD.94.123523. arXiv:1608.00802 [astroph.CO]

Veltmaat J, Niemeyer JC, Schwabe B (2018) Formation and structure of ultralight bosonic dark matter halos. Phys Rev D 98(4):043509. https://doi.org/10.1103/PhysRevD.98.043509. arXiv:1804.09647 [astro-ph.CO]

Veltmaat J, Schwabe B, Niemeyer JC (2020) Baryon-driven growth of solitonic cores in fuzzy dark matter halos. Phys Rev D 101(8):083518. https://doi.org/10.1103/PhysRevD.101.083518. arXiv:1911.09614 [astro-ph.CO]

Visinelli L, Baum S, Redondo J, Freese K, Wilczek F (2018) Dilute and dense axion stars. Phys Lett B 777:64-72. https://doi.org/10.1016/j.physletb.2017.12.010. arXiv:1710.08910 [astro-ph.CO]

Wang L, Dutton AA, Stinson GS, Macciò AV, Penzo C, Kang X, Keller BW, Wadsley J (2015) NIHAO project-I. Reproducing the inefficiency of galaxy formation across cosmic time with a large sample of cosmological hydrodynamical simulations. MNRAS 454(1):83-94. https://doi.org/10.1093/mnras/ stv1937. arXiv:1503.04818 [astro-ph.GA]

Wantz O, Shellard E (2010) Axion cosmology revisited. Phys Rev D 82:123508. https://doi.org/10.1103/ PhysRevD.82.123508. arXiv:0910.1066 [astro-ph.CO]

Watanabe H, Brauner T (2011) On the number of Nambu-Goldstone bosons and its relation to charge densities. Phys Rev D 84:125013. https://doi.org/10.1103/PhysRevD.84.125013. arXiv:1109.6327 [hep-ph]

Watanabe H, Murayama H (2012) Unified description of Nambu-Goldstone Bosons without Lorentz invariance. Phys Rev Lett 108:251602. https://doi.org/10.1103/PhysRevLett.108.251602. arXiv:1203.0609 [hep-th]

Weinberg S (1966) Pion scattering lengths. Phys Rev Lett 17:616-621. https://doi.org/10.1103/ PhysRevLett.17.616

Weinberg S (1978) A new light boson? Phys Rev Lett 40:223-226. https://doi.org/10.1103/PhysRevLett. 40.223 
Weinberg S (1979) Phenomenological Lagrangians. Physica A 96(1-2):327-340. https://doi.org/10.1016/ 0378-4371(79)90223-1

Weinberg S (2009) Effective field theory, past and future. PoS CD 09:001. https://doi.org/10.22323/1.086. 0001. arXiv:0908.1964 [hep-th]

Widrow LM, Kaiser N (1993) Using the Schrödinger equation to simulate collisionless matter. Astrophys J Lett 416:L71-L74

Wigner E (1932) On the quantum correction for thermodynamic equilibrium. Phys Rev 40:749-759. https:// doi.org/10.1103/PhysRev.40.749

Wilczek F (1978) Problem of strong $P$ and $T$ invariance in the presence of instantons. Phys Rev Lett 40:279-282. https://doi.org/10.1103/PhysRevLett.40.279

Yoon JH, Johnston KV, Hogg DW (2011) Clumpy streams from clumpy halos: detecting missing satellites with cold stellar structures. Astrophys J 731:58. https://doi.org/10.1088/0004-637X/731/1/58. arXiv:1012.2884 [astro-ph.GA]

Yoshida N, Sokasian A, Hernquist L, Springel V (2003) Early structure formation and reionization in a warm dark matter cosmology. Astrophys J Lett 591:L1-L4. https://doi.org/10.1086/376963. arXiv:astro-ph/0303622

Zakharov VE, L'vov VS, Falkovich G, (1992) Kolmogorov spectra of turbulence I: wave turbulence. Springer Series in Nonlinear Dynamics. Springer, Berlin. https://doi.org/10.1007/978-3-642-50052-7

Zavala J, Frenk CS (2019) Dark matter haloes and subhaloes. Galaxies 7(4):81. https://doi.org/10.3390/ galaxies7040081. arXiv:1907.11775 [astro-ph.CO]

Zee A (2003) Quantum field theory in a nutshell. Princeton University Press, Princeton

Zel'dovich YB (1971) Generation of waves by a rotating body. JETP Lett 14:180

Zel'dovich YB (1972) Amplification of cylindrical electromagnetic waves reflected from a rotating body. JETP 35:1085

Zhang J, Kuo JL, Liu H, Tsai YLS, Cheung K, Chu MC (2018a) The importance of quantum pressure of fuzzy dark matter on Ly $\alpha$ forest. Astrophys J 863:73. https://doi.org/10.3847/1538-4357/aacf3f. arXiv:1708.04389 [astro-ph.CO]

Zhang J, Tsai YLS, Kuo JL, Cheung K, Chu MC (2018b) Ultralight axion dark matter and its impact on dark halo structure in $N$-body simulations. Astrophys J 853(1):51. https://doi.org/10.3847/15384357/aaa485. arXiv:1611.00892 [astro-ph.CO]

Zhang J, Liu H, Chu MC (2019) Cosmological simulation for fuzzy dark matter model. Front Astron Space Sci 5:48. https://doi.org/10.3389/fspas.2018.00048. arXiv:1809.09848 [astro-ph.CO]

Zhu Q, Marinacci F, Maji M, Li Y, Springel V, Hernquist L (2016) Baryonic impact on the dark matter distribution in Milky Way-sized galaxies and their satellites. Mon Not R Astron Soc 458(2):1559-1580. https://doi.org/10.1093/mnras/stw374. arXiv:1506.05537 [astro-ph.CO]

Zibold T, Nicklas E, Gross C, Oberthaler MK (2010) Classical bifurcation at the transition from Rabi to Josephson dynamics. Phys Rev Lett 105:20. https://doi.org/10.1103/PhysRevLett.105.204101. arXiv: 1008.3057 [cond-mat.quant-gas]

Zinner NT (2011) Vortex structures in a rotating BEC dark matter component. Phys Res Int 2011:734543. https://doi.org/10.1155/2011/734543. arXiv:1108.4290 [astro-ph.CO]

Zolotov A, Brooks AM, Willman B, Governato F, Pontzen A, Christensen C, Dekel A, Quinn T, Shen S, Wadsley J (2012) Baryons matter: why luminous satellite galaxies have reduced central masses. Astrophys J 761:71. https://doi.org/10.1088/0004-637X/761/1/71. arXiv:1207.0007 [astro-ph.CO]

Zwicky F (1933) Die Rotverschiebung von extragalaktischen Nebeln. Helv Phys Acta 6:110-127. https:// doi.org/10.1007/s10714-008-0707-4

Publisher's Note Springer Nature remains neutral with regard to jurisdictional claims in published maps and institutional affiliations. 